\title{
Inherited retinal diseases
}

Citation for published version (APA):

Battu, R. (2018). Inherited retinal diseases: new imaging and molecular genetics. [Doctoral Thesis, Maastricht University]. Maastricht University. https://doi.org/10.26481/dis.20181221rb

Document status and date:

Published: 01/01/2018

DOI:

10.26481/dis.20181221rb

Document Version:

Publisher's PDF, also known as Version of record

\section{Please check the document version of this publication:}

- A submitted manuscript is the version of the article upon submission and before peer-review. There can be important differences between the submitted version and the official published version of record.

People interested in the research are advised to contact the author for the final version of the publication, or visit the DOI to the publisher's website.

- The final author version and the galley proof are versions of the publication after peer review.

- The final published version features the final layout of the paper including the volume, issue and page numbers.

Link to publication

\footnotetext{
General rights rights.

- You may freely distribute the URL identifying the publication in the public portal. please follow below link for the End User Agreement:

www.umlib.nl/taverne-license

Take down policy

If you believe that this document breaches copyright please contact us at:

repository@maastrichtuniversity.nl

providing details and we will investigate your claim.
}

Copyright and moral rights for the publications made accessible in the public portal are retained by the authors and/or other copyright owners and it is a condition of accessing publications that users recognise and abide by the legal requirements associated with these

- Users may download and print one copy of any publication from the public portal for the purpose of private study or research.

- You may not further distribute the material or use it for any profit-making activity or commercial gain

If the publication is distributed under the terms of Article $25 \mathrm{fa}$ of the Dutch Copyright Act, indicated by the "Taverne" license above, 


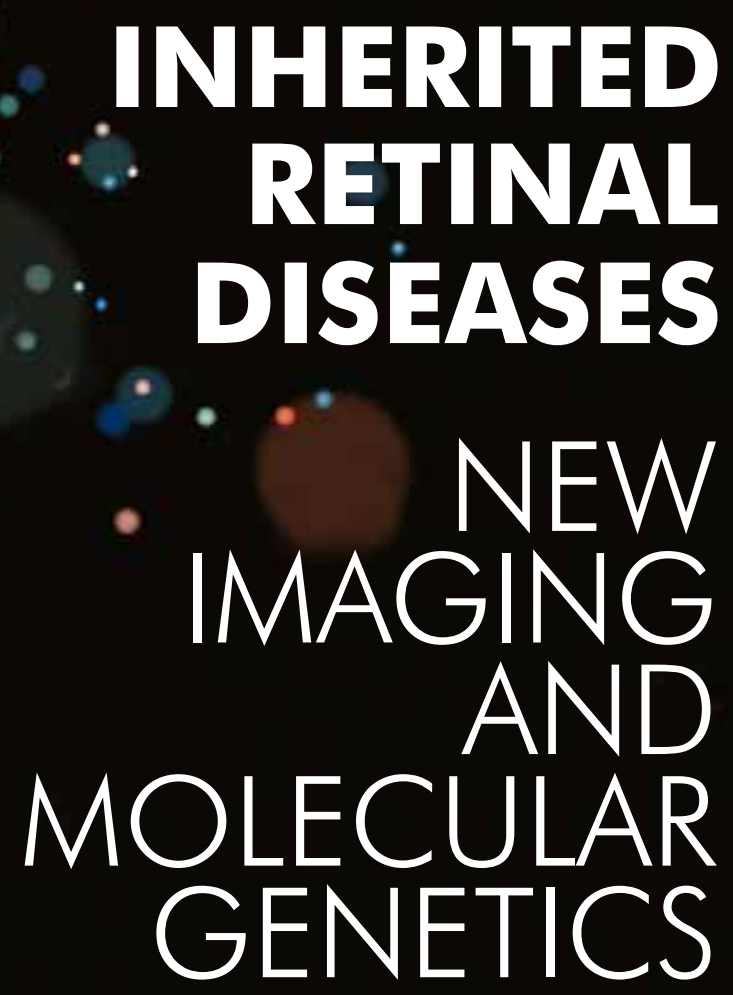

$4.6 \div 5484483$

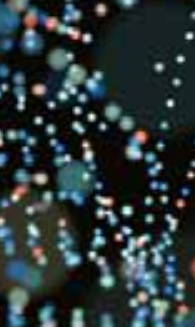

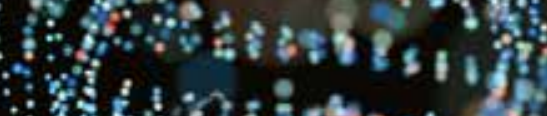

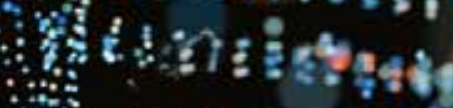

$\because$

$\because$

\%8;

$\%, \%, 42010$

is:

zistis

$=\frac{3 t}{4}$

$2+5=\frac{2}{80}$

$\log _{2}+\cdots$

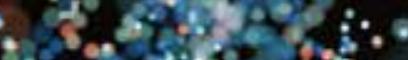

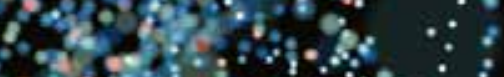

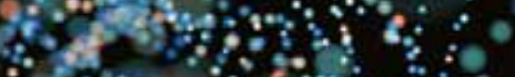

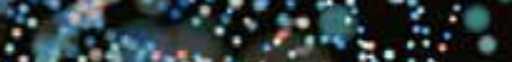





\section{INHERITED RETINAL DISEASES

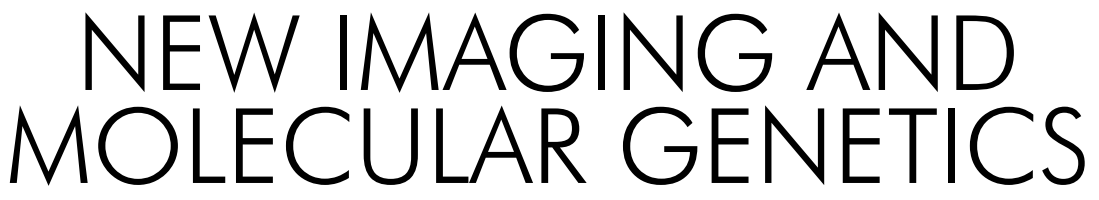

\section{I S SER T A T I O N}

To obtain the degree of Doctor at Maastricht University, on the authority of the Rector Magnificus

PROF. DR. RIANNE M. LETSCHERT

in accordance with the decision of the Board of Deans, to be defended in public on

FRIDAY DECEMBER $21^{\text {ST }} 2018$ at 1145 hours

by

RAJANI BATTU

Born December 23rd, 1970

in Bangalore, India 


\section{SUPERVISOR}

Prof. Dr. C.A.B. Webers

\section{CO-SUPERVISORS}

Dr. J.S.A.G. Schouten

Dr.T.T.J.M. Berendschot

\section{ASSESSMENT COMMITTEE}

Prof. Dr. C.E.M. de Die-Smulders (Chairperson)

Dr. M. Zamani Esteki

Prof. Dr. A. den Hollander (Radboud University, Nijmegen)

Prof. Dr. P. Charbel Issa (University of Oxford, UK)

Prof. Dr. J.E. Wildberger 
Two roads diverged in a wood and I I took the one less travelled by... 



\section{TABLE OF CONTENTS}

1. GENERAL INTRODUCTION

Aims and Outline of Thesis

2. LITERATURE REVIEW

3. ADAPTIVE OPTICS IMAGING OF THE RETINA Indian J Ophthalmol 2014;62(1):60-5.

4. ADAPTIVE OPTICS IMAGING OF THE OUTER RETINAL

TUBULES IN BIETTI'S CRYSTALLINE DYSTROPHY

Eye (Lond) 2016: 30(5); 705-12

5. CORRELATION OF STRUCTURE AND FUNCTION OF THE MACULA IN PATIENTS WITH RETINITIS PIGMENTOSA

Eye (Lond) 2015: 29(7); 895-901

6. RETINAL OXYGEN SATURATION IN RETINITIS PIGMENTOSA AND MACULAR DYSTROPHIES IN ASIAN INDIAN EYES IOVS 2015:56(5); 2798-802

7. IDENTIFICATION OF NOVEL MUTATIONS IN ABCA4 GENE: CLINICAL AND GENETIC ANALYSIS OF INDIAN PATIENTS WITH STARGARDT DISEASE BMRI 2015:2015;1-10

8. EXOME SEQUENCING IS AN EFFICIENT TOOL FOR VARIANT LATEINFANTILE NEURONAL CEROID LIPOFUSCINOSIS MOLECULAR DIAGNOSIS PLoS One. 2014 Oct 15; 9(10) 
9. GENETIC ANALYSIS AND CLINICAL PHENOTYPE OF TWO INDIAN FAMILIES WITH X-LINKED CHOROIDEREMIA Indian J Ophthalmol 2016;64(12):924-29.

10. CHALLENGES OF MANAGING RETINAL DYSTROPHIES: AN EXPERIENCE FROM SOUTH INDIA.

Ophthalmic Genet. 2018: 39(1);1-3

11. METHODS TO DETECT PROGRESSION IN RETINITIS PIGMENTOSA: A REVIEW In Submission

12. GENERAL DISCUSSION

13. SUMMARY 
CHAPTER 1

\section{GENERAL INTRODUCTION}

AIMS AND OUTLINE OF THE THESIS 



\section{General Introduction}

Retinal degenerative disorders or inherited retinal diseases (IRD) are a group of heterogeneous diseases that are characterized by inherited, progressive photoreceptor dysfunction, photoreceptor cell loss and eventual atrophy of retinal tissue. They are a significant cause of bilateral blindness worldwide. First described in 1857 by Donders', a Dutch ophthalmologist, they were initially identified by correlating clinical findings with family pedigrees and inheritance. More recently, electrophysiological studies and newer retinal imaging techniques have helped identify functional impact and its cellular causes for visual disability. Advances in molecular genetics and the recent possibility of gene and cell-based therapies have opened up new vistas in the management of these disorders.

Retinitis Pigmentosa (RP) is the most common type of inherited retinal degenerative disease. It is a progressive inherited retinal dystrophy characterized by night blindness, bone spicule-like pigmentary changes in the midperiphery of the retina, progressive loss of peripheral vision, and decreased or undetectable responses on an electroretinogram. ${ }^{2,3}$ When the disease is associated with systemic abnormalities like deafness, polydactyly, renal disease, it is referred to as syndromic RP. Syndromic RP includes a large array of diseases like Usher syndrome, Bardet Bidel syndrome, Joubert's syndrome amongst others. The prevalence of non-syndromic RP has been reported to vary from a conservative estimate of 1 in 3500 in the United States ${ }^{4}$ to as high as 1 in 930 in southern India. ${ }^{5}$

Stargardt disease or fundus flavimaculatus is the most common juvenile macular dystrophy and a common cause of central vision loss in adults under age of 50, with an estimated prevalence of $1: 8,000$ to $1: 10,000.6,7$ Leber Congenital Amaurosis (LCA) is an infantile, autosomal recessive variant of retinitis pigmentosa that was first described by Leber in 1869. The first successful gene therapy trials in ophthalmology have been conducted in patients with LCA, and the Food and Drug Administration (FDA) has recently approved a phase-3 drug for LCA with RPE65. ${ }^{8,9}$

New imaging techniques and functional tests that are used to examine and study patients with retinal dystrophies have evolved. These include spectral-domain optical coherence tomography (SD-OCT), ${ }^{10}$ fundus autofluorescence (FAF), ${ }^{11}$ micro-perimetry 
(MP), ${ }^{12}$ and adaptive optics (AO). ${ }^{13}$ Although these imaging techniques and functional tests have been used in several studies, their clinical relevance and opportunities for use in clinical research have not yet been elucidated in much detail. Clinical research on these new imaging techniques and functional tests contribute to identify their clinical value in these domains. This clinical value is known when the new imaging techniques contribute to improved diagnosis, prognosis, and prediction of therapy effect or monitoring of the disease.

Management of inherited retinal diseases has conventionally been limited to genetic counselling and low-vision rehabilitation. Through recent scientific advancements, genotyping is now at the forefront of disease management. Although there is no effective intervention for this group of diseases, several clinical trials are underway to either slow down or arrest the progression of the disease by gene therapy, or cellbased therapies. ${ }^{14-18}$

\section{Aims and Outline of the Thesis}

There is a paucity of clinical and genetic data from India in the field of inherited retinal diseases. Given the population and the higher prevalence of these conditions in India, ${ }^{5}$ there is a need to understand the burden of these diseases in the population. This becomes especially imperative in the wake of extensive research into this field around the world and rapidly developing clinical trials. With progress in technology, newer imaging techniques are becoming available and these are especially useful in designing clinical trials and critical end points of treatment.

The current thesis therefore aims to explore how new imaging techniques contribute to the evaluation of patients with IRDs. Part of this work was done at the genetic eye clinic at Narayana Nethralaya, a large tertiary referral hospital and at the Centre for Eye Genetics and Research, both in Bangalore, India.

Some of the patients underwent genetic testing, and we present the genetic data from these patients and their families. These data were not limited to the assessment of existing polymorphisms but also let to the discovery of new polymorphisms. Note that these findings are not limited to patients from India, but contribute to the understanding of inherited retinal diseases in general. 


\section{The thesis is outlined below in 12 chapters.}

CHAPTER TWO is a short literature review on imaging and genetics of retinal dystrophies and a more elaborate review of the relevant literature from India.

CHAPTER THREE gives an overview and describes the application of Adaptive Optics in retinal imaging.

CHAPTER FOUR describes the outer retinal tubules on the Adaptive Optics in patients with Bietti's crystalline dystrophy. Currently, the knowledge of these tubules is mostly from studies involving SD-OCT. These tubules appear as hyporeflective ovoid spaces with hyperreflective borders in the outer nuclear layers on the OCT. They were initially described in AMD and have been proposed as a rearrangement of the photoreceptor layer in response to injury. ${ }^{19}$ This is the first study to demonstrate and describe these tubules in detail with adaptive optics in Bietti's crystalline dystrophy.

CHAPTER FIVE describes the relation of macular structure and function in patients with retinitis pigmentosa. The ultrastructure on the spectral domain optical coherence tomography was related to function as measured by microperimetry (MAIA, Macular integrity assessment). It led to the identification of OCT changes that cause a reduction in function.

CHAPTER SIX describes a cross-sectional study of the oxygen saturation profiles of patients with RP and macular dystrophy using the Oxymap T1 retinal oximeter. Disturbed ocular blood flow, increased arterio-venous transit time and reduced blood flow velocities are prominent features of RP and macular dystrophies. This study found a decrease in vascular diameters, an increase in arteriolar and venous saturation and arterio-venous saturation difference in the RP group. ${ }^{20,21}$ The study also showed alteration in oxygen saturation profiles in all quadrants in the RP group but mainly in the infero-temporal quadrant in the macular group. Studies like these are important in understanding the pathobiology of these diseases.

CHAPTER SEVEN is a study of the clinical and genetic analysis of patients with Stargardt disease from India and describes new mutations in the ABCA4 gene. The study identified five mutations in the ABCA4 gene, two of which are novel. This is the first study on genetic analysis of patients with Stargardt disease from India and adds to the spectrum of disease-causing mutations. 
CHAPTER EIGHT describes the results of exome sequencing in a family clinically presenting with visual failure and seizures. Neuronal ceroid lipofuscinosis or Batten disease is a childhood onset neurodegenerative disease, that often presents to the ophthalmologist first. ${ }^{22}$ We identified two novel MFSD8 mutations in the family. The chapter discusses the importance of exome sequencing in consanguineous families with neuronal ceroid lipofuscinosis.

CHAPTER NINE is a clinical and genetic study of two families identified with Choroideremia. SD-OCT showed loss of outer retina, preservation of the inner retina and choroidal thinning in the affected males and retinal pigment epithelial changes in the female carriers. Two known mutations were identified in the CHM gene. This is the first study to describe the genetic analysis of patients with Choroideremia from India.

CHAPTER TEN addresses the challenges that patients and caregivers face in the management of retinal dystrophies. India faces several difficulties including lack of genetic counsellors and inadequate access to molecular genetic testing. Associations like the 'Organization for Rare Diseases India' are making efforts to bridge these gaps. 23

CHAPTER ELEVEN reviews all the current and newer methods used to assess progression of RP. With many clinical trials and therapeutic interventions on the anvil, being able to monitor the progression and identify critical end points for these trials becomes crucial.

CHAPTER TWELVE is a general discussion of inherited retinal dystrophies and the current status of various therapeutic approaches. The challenges associated with identifying the timing of intervention, need for multiple modalities of imaging to identify suitable end points and the contribution of this thesis to the knowledge base of IRDs is discussed.

CHAPTER THIRTEEN summarizes the various studies and its significance in clinical practice. It emphasises a clinical approach in inherited retinal diseases especially with relevance to India. 


\section{References}

1. Donders FC. Beiträge zur pathologischen Anatomie des Auges Pigmentbildung in der Netzhaut. Archiv für Ophthalmologie 1857;3:139-65

2. Fishman GA. Retinitis pigmentosa. Genetic percentages. Arch Ophthalmol 1978;96:822-6.

3. Hamel C. Retinitis pigmentosa. ORP hanet J Rare Dis 2006; 1:40

4. Bunker $C H$, Berson EL, Bromley WC, Hayes RP, Roderick TH. Prevalence of retinitis pigmentosa in Maine. Am J Ophthalmol 1984;97:357-65

5. Sen $P$, Bhargava A, George $R$, et al. Prevalence of retinitis pigmentosa in South Indian population aged above 40 years. Ophthalmic Epidemiol 2008;15:279-81

6. Weleber RG. Stargardt's macular dystrophy. Arch Ophthalmol 1994;112:752-4.

7. Walia S, Fishman GA. Natural history of phenotypic changes in Stargardt macular dystrophy. Ophthalmic Genet 2009;30:63-8.

8. Bainbridge JWB, Smith AJ, Barker SS, et al. Effect of gene therapy on visual function in Leber's congenital amaurosis. NEJM 2008;358:2231-39

9. Lee H, Lotery A. Gene therapy for RPE65-mediated inherited retinal dystrophy completes phase 3. Lancet 2017. Aug 26;390(10097):823-824

10. Fischer M, Fleischhauer JC, Gillies MC et al. A new method to monitor visual field defects caused by photoreceptor degeneration by quantitative optical coherence tomography. Invest Ophthalmol Vis Sci 2008; 49:3617-3621.

11. Robson AG, Michaelides M, Saihan Z, et al. Functional characteristics of patients with retinal dystrophy that manifest abnormal parafoveal annuli of high density fundus autofluorescence; a review and update. Doc Ophthalmol 2008;1 16:79-89.

12. Rohrschneider KS, Bultmann and Springer C. Use of fundus perimetry (microperimetry) to quantify macular sensitivity. Prog Retin Eye Res 2008; 27: 536-48

13. Lombardo M, Lombardo G, Ducoli P, Serrao S. Adaptive optics photoreceptor imaging. Ophthalmology 2012;119:1498

14. Bennett J. Gene therapy for retinitis pigmentosa. Curr Opin Mol Ther 2000; 2: 420-5.

15. Tan MH, Smith AJ, Pawlyk B, et al. Gene therapy for retinitis pigmentosa and Leber congenital amaurosis caused by defects in AIPL 1: effective rescue of mouse models of partial and complete Aip/1 deficiency using AAV2/2 and AAV2/8 vectors. Hum Mol Genet 2009;18: 2099-114. 
16. Humayun MS, Dorn JD, da Cruz L, et al. Argus II Study Group. Interim results from the international trial of Second Sight's visual prosthesis. Ophthalmology 2012;119:77988.

17. Chader GJ, Weiland J and Humayun MS. Artificial vision: needs, functioning, and testing of a retinal electronic prosthesis. Prog Brain Res 2009;175:317-32.

18. MacLaren RE, Groppe M, Barnard AR, et al. Retinal gene therapy in patients with choroideremia: initial findings from a phase 1/2 clinical trial. Lancet 2014;383:112937.

19. Zweifel SA, Engelbert M, Laud K, Margolis R, Spaide RF, Freund KB. Outer retinal tubulation: a novel optical coherence tomography finding. Arch Ophthalmol 2009; 127:1596-602.

20. Konieczka K, Flammer AJ, Todorova M, et al. Retinitis pigmentosa and ocular blood flow. EPMA J 2012;3:17.

21. Wolf S, Pöstgens $H$, Bertram B, et al. Hemodynamic findings in patients with retinitis pigmentosa. Klin Monbl Augenheilkd 1991;199:325-329

22. Haltia M. The neuronal ceroid-lipofuscinoses. J Neuropathol Exp Neurol 2003; 62: $1-13$

23. Rajasimha HK, Shirol PB, Ramamoorthy $P$, et al. Organization for rare diseases India (ORDI) - addressing the challenges and opportunities for the Indian rare diseases' community. Genet Res (Camb). 2014;96:1-10. 
CHAPTER 2

\section{LITERATURE REVIEW}



Genomic medicine has progressed rapidly after the successful completion of the Human Genome Project, ${ }^{1}$ Hapmap Project ${ }^{2}$ and Genome Wide Association Study. ${ }^{3}$ It has had a major impact in ophthalmology, especially for the identification of genes involved in the pathogenesis of retinal dystrophies. Retinal dystrophies are a group of inherited, retinal degenerative diseases that cause progressive loss of photoreceptors and/or retinal pigment epithelium resulting in partial or complete loss of vision.

Retinitis pigmentosa is the commonest retinal dystrophy, which is characterized by night blindness and progressive loss of peripheral visual fields. The prevalence of RP ranges from 1 in 3500 in the US ${ }^{4}$ to 1 in 1000 in China ${ }^{5}$ and 1 in 750-930 patients in India. ${ }^{6,7}$ The disease is very heterogeneous in nature, with varied age of presentation and clinical manifestation. Clinically, prominent bony spicules, arteriolar attenuation and waxy pallor of the optic nerve head characterize the disease (Figure 1).

FIGURE 1.

Fundus picture of the right and left eyes of a 48-year old patient with RP showing arteriolar attenuation and bony spicules.

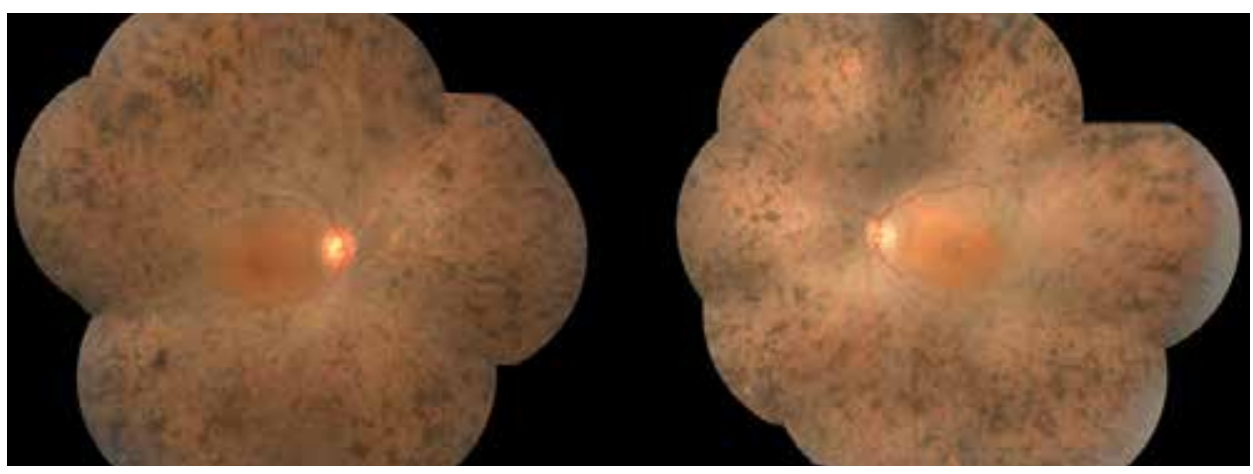

Progression of the disease is monitored by measuring the ring of autofluorescence on fundus autofluorescence (FAF) (Figure 2), measuring the inner segment/outer segment layer on the spectral domain optical coherence tomography (SD-OCT) (Figure 3) and visual fields. 
FIGURE 2.

Fundus autofluorescence at the macula of the same patient as in Figure 1. Note the ring of hyperautofluorescence (white arrows) surrounding an area of hypo and normal autofluorescence.

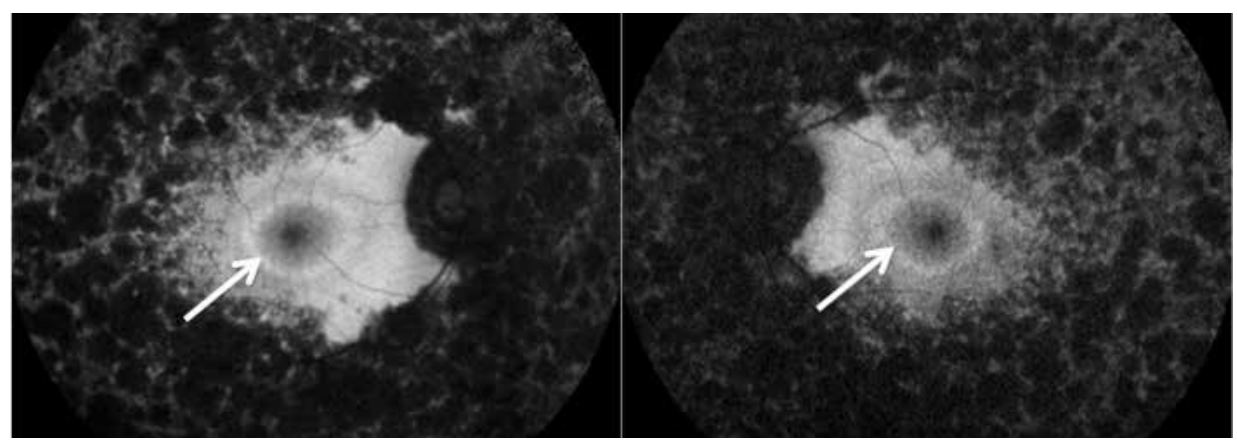

\section{FIGURE 3.}

SD-OCT of the macula as the same patient as in figure 1 . Note the preserved central area of retained IS/OS segments (white arrows).
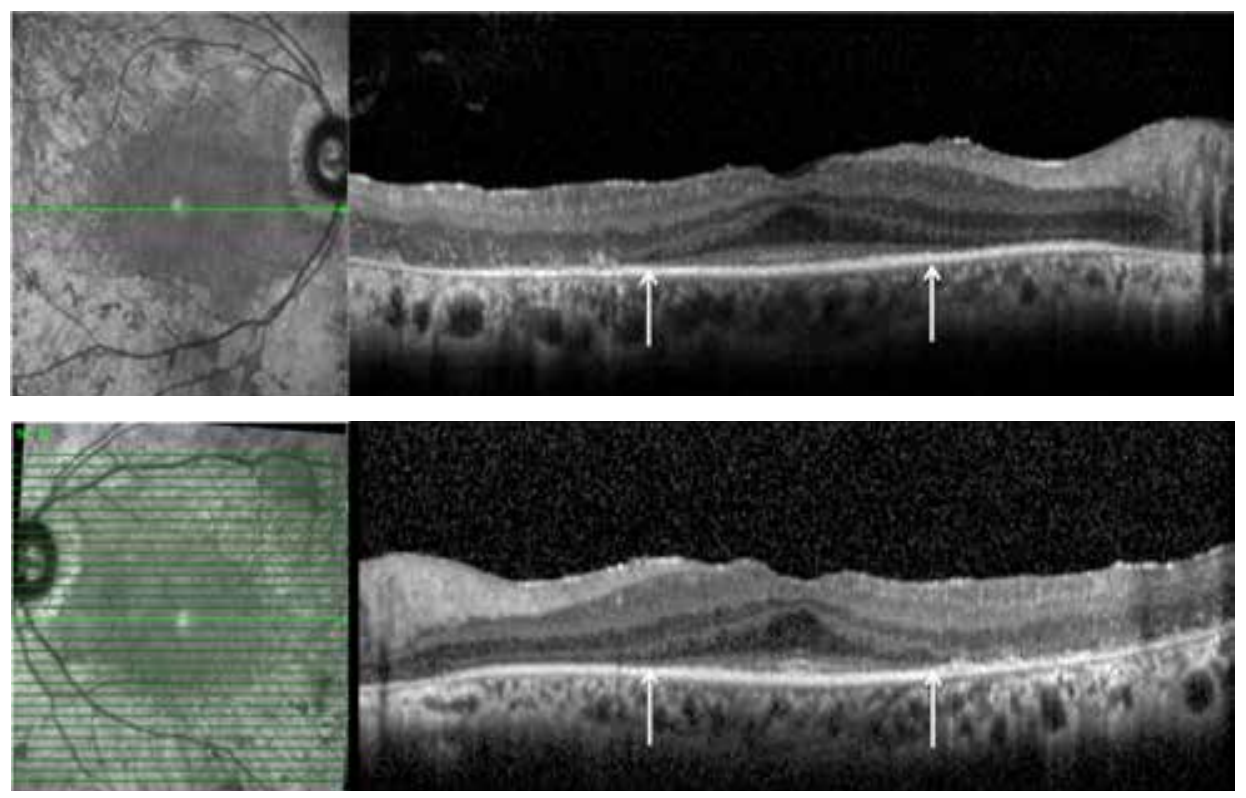
RP mostly occurs as an isolated disease (nonsyndromic) or in association with other systemic abnormalities (syndromic). Several syndromic variants of RP exist, all of which are transmitted in an autosomal recessive pattern. The common ones include Usher's syndrome and Bardet Biedl syndrome. Non-syndromic RP may be transmitted in all three Mendelian forms: autosomal dominant (AD), autosomal recessive (AR) and X-linked recessive $(\mathrm{XL})$; however nearly half the cases are isolated or sporadic in nature..$^{8-10}$

To date, mutations in over 84 genes have been identified causing non-syndromic RP. ${ }^{11}$ Each of these genes encodes a protein that plays an important role in processes like the phototransduction or visual cycle occurring within the neuroretina and/or RPE or the connecting cilia. Therefore, a mutation in a gene within a specific pathway can cause the whole pathway to become impaired or even disrupted entirely. The first mutation identified for RP was the rhodopsin gene on chromosome 3, which now has over 100 known variants. The rhodopsin gene still accounts for only about $10 \%$ of the RP variants, and over half of the gene mutations are yet unknown. ${ }^{12}$

Although no definitive treatment exists for the retinal degeneration seen in typical RP or most of its syndromic variants, there has been tremendous progress in this direction in the last decade. Treatment modalities hither-to have mostly included high dose vitamin A supplement and anti-oxidants. ${ }^{13}$ Topical and systemic acetazolamide therapy has been tried for cystoid macular edema, although their role remains unclear. ${ }^{14}$

A highlight in the visual rehabilitation of end stage RP in recent years has been the development of retinal prosthetic implant, the Argus II. ${ }^{15}$ Clinical trials with retinal prostheses have shown some improvement in visual recovery. Several clinical trials are underway that have tried placing the electrodes in the epiretinal, subretinal, suprachoroidal space or in the optic nerve head. ${ }^{16,17}$

Leber Congenital Amaurosis (LCA) is an infantile, autosomal recessive variant of retinitis pigmentosa that was first described by Leber in 1869. Characteristic features include severe vision loss from infancy, nystagmus, and sluggish pupillary response. The fundus usually has a normal appearance, although findings such as salt and pepper pigment mottling, macular coloboma-like appearance, and retinal flecks have been reported. High hyperopia is also a common finding in LCA patients. ${ }^{18-20}$

Till date, mutations in 25 genes currently associated with LCA. The most common mutations include CEP290 (15\%), GUCY2D (12\%), CRB1 (10\%) and RPE65 (6\%). ${ }^{21}$ Patients with LCA are among the first group of individuals with hereditary retinal disease who 
have therapeutically benefited from improved molecular understanding of their disease. Gene therapy for LCA was first studied in canines through the replacement of the RPE65 gene through a subretinal injection of an adeno-associated viral gene vector. Phase 1 studies showed potential benefit of gene replacement in RPE65mediated LCA and a subjective improvement in visual perception, and a statistically significant improvement in the dark-adapted ERG response. A phase 3 study showed significant improvement in the standardized multi-luminance mobility testing with Voretigene neparvovec gene replacement in patients with RPE65 mediated retinal dystrophy. ${ }^{22,23}$ In December 2017, the USFDA approved the gene therapy 'Luxturna' ((voretigene neparvovec-rzyl), developed by by Spark Therapeutics, to treat children and adults with biallelic REP65 mutation-associated retinal dystrophy.

Stargardt disease or fundus flavimaculatus is the most common juvenile macular dystrophy and a common cause of central vision loss in adults under age of 50 , with an estimated prevalence of 1:8,000 to 1:10,000. ${ }^{24}$ The vast majority of cases are autosomal recessive. Affected individuals may present with decreased central vision in the first two decades of life. Fundus findings are variable and include mottling or atrophy of the retinal pigment epithelium, bull's eye maculopathy, beaten-bronze macular appearance, and yellow-white pisciform flecks in the perifoveal or peripheral retina (Figure 4). Vision may progressively deteriorate to the level of 20/50 to 20/200 and may show no correlation with fundus appearance. ${ }^{25}$ 
FIGURE 4.

Fundus details of a patient with Stargardt. Fig A shows the fundus picture of the right eye. Note the central macular atrophy(black arrow). Fig B shows the FAF of the same eye. Note the central area of hypoautofluorescence with a surrounding ring of hyperautofluorescence (white arrow). Fig ( shows the spectral domain $0 \mathrm{CT}$ of the same eye. Note the foveal thinning (blue arrows) and the central loss of photoreceptors and the external limiting membrane (red arrows)
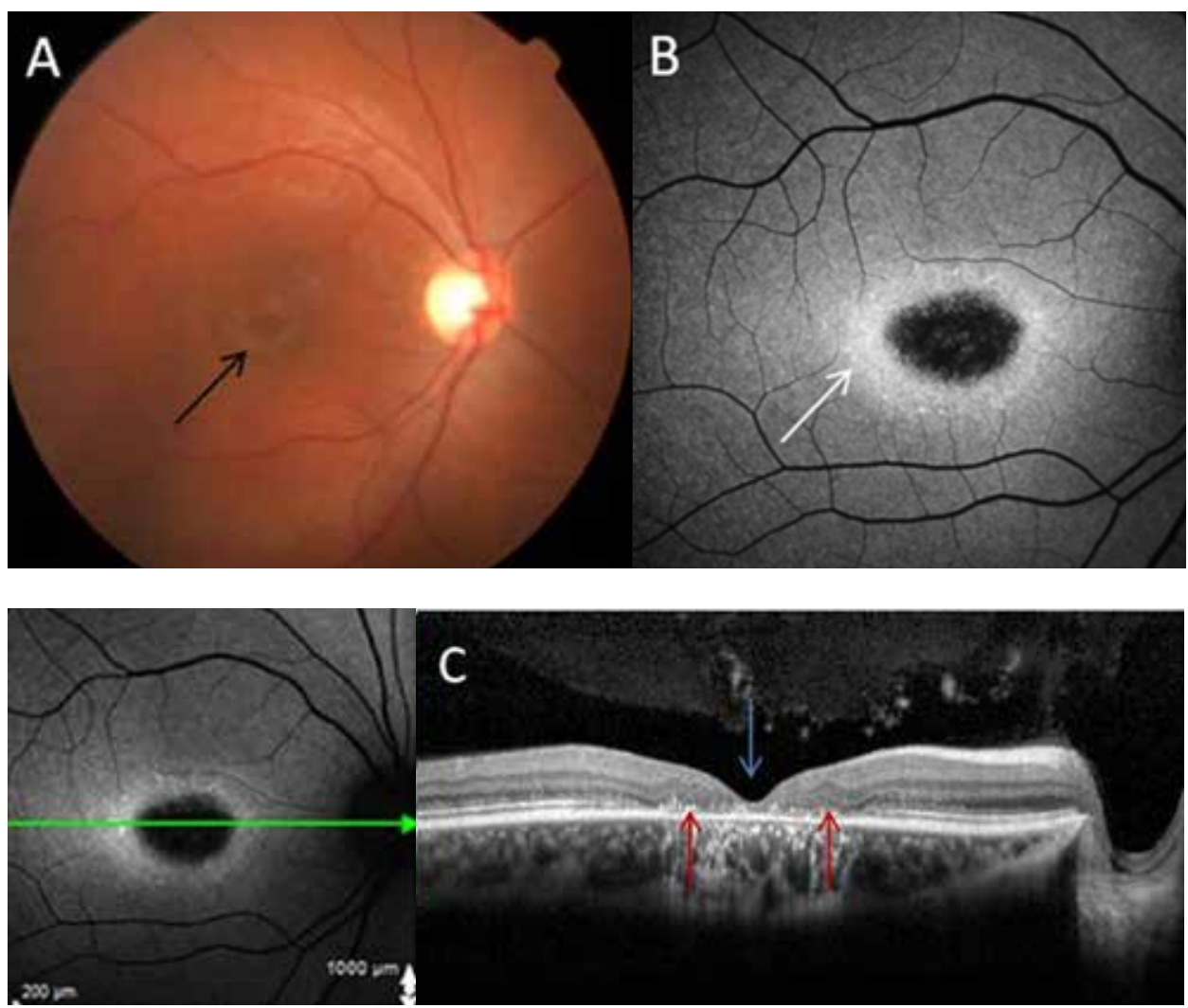

The terms Stargardt disease and fundus flavimaculatus have sometimes been used interchangeably, although more specific correlation with their individual phenotypes has been suggested. Stargardt disease preferentially refers to the early onset disease associated with vision loss, beaten-metal macular appearance, and few surrounding perimacular flecks. Fundus flavimaculatus describes adult-onset disease with a better presenting visual acuity and diffuse peripheral flecks. Molecular studies have confirmed the genetic similarities of these 2 phenotypes on chromosomes $1 \mathrm{p} 13$ and $6 \mathrm{p} 21 .{ }^{24}$ 
Pathogenesis of Stargardt disease is linked to an abnormal accumulation of lipofuscin in the RPE in the early stages, followed by slowing of the rod and cone retinoid cycles later in the disease. Genes identified for Stargardt disease include ABCA4 (chromosome 1p13-21) for the ABCR retinal ATP transporter protein; CNGB3 (chromosome 8q2122) for a cyclic nucleotide gated channel on cone plasma membranes; PRPH2 (chromosome 6p21) for the human peripherin/RDS protein involved in photoreceptor disc morphogenesis; STGD4 (chromosome 4p15); and the rare autosomal dominant mutation ELOVL4 (chromosome 6q14). 26,27

Although definitive therapy is yet unavailable for Stargardt disease, significant progress through genetic, functional, cellular and translational studies has allowed major advances in therapeutic applications of these diseases. ${ }^{28,29}$

Choroideremia is a X-linked disorder that leads to progressive degeneration of the photoreceptors, retinal pigment epithelium and choriocapillaris. Symptoms include night blindness and progressive constriction of visual fields; most affected males are legally blind by their midlife. The causative gene has been mapped at Xq21.1-q21.3; mutations in this gene leads to the loss of loss of function of a protein necessary for retinal cell health, Rab Escort Protein 1 (REP1). The disease has characteristic fundus features that includes progressive atrophy of the retina and choroid..$^{30}$

Choroideremia has several attributes that make it an ideal target for gene augmentation therapy. ${ }^{31}$ Gene therapy was successfully administered in patients with CHM in a multicenter clinical trial, with consistent improvement in rod and cone function. ${ }^{32}$

Genetic testing allows rapid, cost-effective testing for several known mutations through a small DNA sample placed on a biochip. These could be targeted gene sequencing, whole exome sequencing or whole genome sequencing. Advantages of DNA analysis include the identification of new genes, protein products, and their roles in the retinal visual pathways. This also helps confirm diagnoses, use genotypephenotype correlations to identify disease features, provide prognostic information, and allow future treatment options. The role of genetic testing has therefore progressed from an elective means of confirming a diagnosis to a critical element in the management of these complex dystrophies. 


\section{Status of Genetic Eye Research in India}

Eye genetics is a nascent field and an emerging specialty in Ophthalmology in India. Retinitis pigmentosa and Retinoblastoma are the common eye genetic disorders seen in any major eye hospital in India.

The prevalence of RP in India has been much higher than that noted in the Western population. Sen et al. estimated the prevalence of RP as 1 in 930 in the urban population and 1 in 372 in the rural general population of Southern India, aged 40 years and above. ${ }^{6}$ Nangia et al. estimated the prevalence of RP as 1 in 750 in the adult population of rural Central India. ${ }^{7}$

This higher prevalence could be attributed to an increased prevalence of consanguineous marriages in the country, especially the southern part of India. ${ }^{33}$ Data from the National Family Health Survey (NFHS II) in India showed that $34.7 \%$ of marriages in south India are consanguineous; $26.2 \%$ of women married close blood relatives and $8.5 \%$ women married distant blood relatives. ${ }^{34} \mathrm{~A}$ higher proportion of autosomal recessive inheritance has been described from India. ${ }^{35,36}$

One of the earliest reports on RP from India was in 1995 from 78 families; 9\% were autosomal dominant, 36\% autosomal recessive, 3\% X-linked recessive, 44\% were isolated cases and $8 \%$ were of undetermined genetic type. ${ }^{36}$ Consanguinity was a prominent feature of the families. A segregation analysis of four hundred families from 16 different states in India found that majority (75\%) of families were autosomal recessive. ${ }^{37}$ Homozygosity mapping was used to identify mutations in 34 families that revealed mutations in RPE65, RP1, TULP1, RLBP1, and ABCA4 genes. ${ }^{38}$

There are currently no prevalence studies of Stargardt disease in India. The prevalence of LCA is about 2 to 3 per 100,000 live births, ${ }^{39}$ its prevalence appears to be higher in consanguineous population like south India. ${ }^{33}$ Considering the success of gene therapy, there is a lot of interest in diagnosis of RPE65 LCA. Ramprasad et al. reported a splicesite mutation in LCA5 in 2 sisters from a consanguineous family. ${ }^{40}$ Sundaresan et al. in 2008 identified 177 unique LCA causing mutations in ${ }^{41}$ subjects, and concluded that the mutations commonly seen in North America are rare in the Indian population, and suggested that novel mutations may be responsible. ${ }^{41}$ Verma et al. screened 30 subjects with LCA for causative genes; 11 cases (36.6\%) revealed pathogenic variations with the 
involvement of RPE 65(16.6\%), GUCY2D(10\%), RPGRIP (3.3\%), RPGRIP1 (3.3\%), AIPL1 (3.3\%) and CRX \& IQCB1 (3.3\%). ${ }^{42}$ Srilekha et al. identified the following mutations in eleven consanguineous familes with LCA; AIPL1- 3 families, RPE65- 2 families, GUCY2D, CRB1, RDH12, IQCB1 and SPATA7 in one family each, respectively. ${ }^{43}$ It is of interest to note that all the mutations reported above are from the southern part of India.

Considering the burden of retinal dystrophies in India, there is a need for major research into this area. Mutations amongst the Indian population may be different than the rest of the world as has been shown to some extent. Moreover, the high rate of consanguinity may reveal more rare mutations. It is imperative that studies are done to understand these diseases in India better and identify the specific genes and mutations involved. Gene therapy needs to be specific for India to address the specific genes and mutations.

\section{References}

1. Lander ES, Linton LM and Birren B. Initial sequencing and analysis of the human genome. Nature 2001;409:860-921.

2. International Hapmap Consortium. A haplotype map of the human genome. Nature 2005;437:1299-320.

3. Hirschhorn JN and Daly MJ. Genome wide association studies for common diseases and complex straits. Nat Rev Genet 2005;5:95-108.

4. Bunker $\mathrm{CH}$, Berson EL, Bromley WC, Hayes RP, Bunker C, Berson E. Prevalence of retinitis pigmentosa in Maine. Am J Ophthalmol 1984;97:357-65.

5. $X u L, H u L, M a K, L i J$ and Jonas JB. Prevalence of retinitis pigmentosa in urban and rural adult Chinese: The Beijing Eye Study. Eur J Ophthalmol 2006;16:865-6

6. Sen P, Bhargava A, George R et al. Prevalence of retinitis pigmentosa in South Indian population aged above 40 years. Ophthalmic Epidemiol 2008;15:279-81.

7. Nangia $V$, Jonas JB, Khare A, Sinha A Prevalence of RP in India: the central India Eye and Medical Study. Acta Ophthalmol 2012; 1987:1-2

8. Fishman GA. Retinitis pigmentosa. Genetic percentages. Arch Ophthalmol 1978;96:822-6.

9. Hamel C, Hartong DT, Berson EL, Dryja TP and Hamel CP. Retinitis pigmentosa. Orphanet J Rare Dis 2006; 368:1795-1809

10. Bonneau D, Frézal J, Munnich A, Dufier JL, Kaplan J. Clinical and genetic heterogeneity in retinitis pigmentosa. Hum Genet1990;85:635-42. 
11. Verbakel SK, van Huet RAC, Boon CJF, et al. Non-syndromic retinitis pigmentosa. Prog Retin Eye Res. 2018 Mar 27.

12. Reynolds JD, Olitsky S Eds. Generalized Inherited Retinal Dystrophies. In Pediatric Retina. Springer-Verlag Berlin Heidelberg; 2010; 295-313

13. Berson EL, Rosner B, Sandberg MA et al. A randomized trial of vitamin $A$ and vitamin $E$ supplementation for retinitis pigmentosa. Arch Ophthalmol 1993;111:761-72.

14. Fishman GA, Gilbert LD, Fiscella RG, Kimura AE, Jampol LM. Acetazolamide for treatment of chronic macular edema in retinitis pigmentosa. Archives of ophthalmology 1989;107:1445-1452

15. Chader GJ, Weiland J, Humayun MS. Artificial vision: needs, functioning, and testing of a retinal electronic prosthesis. Progress in Brain Research 2009;175:317-332

16. Weiland JD, Cho AK, Humayun MS. Retinal prostheses: Current clinical results and future needs. Ophthalmology 2011;118:2227-37.

17. Sakaguchi H, Kamei M, Fujikado T et al. Artificial vision by direct optic nerve electrode (AV-DONE) implantation in a blind patient with retinitis pigmentosa. J Artif Organs 2009;12:206-9.

18. Pinckers AJL. Leber's congenital amaurosis as conceived by Leber. Ophthalmologica 1979;179:48-51.

19. Fazzi E, Signorini SG, Scelsa B, Bova SM, Lanzi G. Leber's congenital amaurosis: An update. Eur J Paediatr Neurol 2003;7:13-22.

20. Wagner RS, Caputo AR, Nelson LB, Zanoni D. High hyperopia in Leber's congenital amaurosis. Arch Ophthalmol 1985;103:1507-9.

21. Kumaran N, Moore AT, Weleber RG and Michaelides M. Leber congenital amaurosis/ early-onset severe retinal dystrophy: clinical features, molecular genetics and therapeutic interventions. Br J Ophthalmol. 2017;101(9):1147-1154.

22. Bainbridge JWB, Smith AJ, Barker SS et al. Effect of gene therapy on visual function in Leber's congenital amaurosis. N Engl J Med 2008;358:2231-9.

23. Russell S, Bennett J, Wellman JA et al. Efficacy and safety of voretigene neparvovec (AAV2-hRPE65V2) in patients with RPE65-mediated inherited retinal dystrophy: a randomised, controlled, open-label, phase 3 trial. Lancet 2017; 390: 849-60.

24. Genead MA, Fishman GA, Stone EM, Allikmets $R$. The natural history of stargardt disease with specific sequence mutation in the ABCA4 gene. Invest Ophthalmol Vis Sci. 2009;50:5867-71.

25. Walia S, Fishman GA. Natural history of phenotypic changes in Stargardt macular dystrophy. Ophthalmic Genet. 2009;30:63-8. 
26. Koenekoop RK. The gene for Stargardt disease, ABCA4, is a major retinal gene: a mini-review. Ophthalmic Genet. 2003;24:75-80.

27. Kniazeva M, Chiang MF, Morgan B, Anduze AL, Zack DJ, Han M, Zhang K. A new locus for autosomal dominant stargardt-like disease maps to chromosome 4. Am J Hum Genet. 1999;64:1394-9.

28. Sears AE, Bernstein PS, Cideciyan AV, et al. Towards Treatment of Stargardt Disease: Workshop Organized and Sponsored by the Foundation Fighting Blindness. TransI Vis Sci Technol. 2017 Sep 14;6(5):6.

29. Chuang K, Fields MA and Del Priori LV. Potential of Gene Editing and Induced Pluripotent Stem Cells (iPSCs) in Treatment of Retinal Diseases. Yale journal of Biology and Medicine. 90 (2017), pp.635-642. .

30. Coussa RG, Traboulsi El. Choroideremia: A review of general findings and pathogenesis. Ophthalmic Genet 2012;33:57-65.

31. Vasireddy V, Mills JA, Gaddameedi R et al. AAV-mediated gene therapy for choroideremia: preclinical studies in personalized models. PLoS One 2013;8:e61396.

32. MacLaren RE, Groppe M, Barnard AR et al. Retinal gene therapy in patients with choroideremia : initial findings from a phase 1 / 2 clinical trial. Lancet 2014;383:1129-1137.

33. Kumaramanickavel G, Joseph B, Vidhya A, Arokiasamy T Shetty SN, Consanguinity and ocular genetic diseases in South India: analysis of a five-year study. Community Genet 2002;5:182-5.

34. Audinarayana $N$, Krishnamoorthy S. Contribution of social and cultural factors to the decline in consanguinity in south India. Soc Biol 2000;47:189-200.

35. Vinchurkar MS, Sathye SM, Dikshit M. Retinitis pigmentosa genetics: a study in Indian population. Indian J Ophthalmol 1996;44:77-82.

36. Kar B, John S, Kumaramanickavel G. Retinitis pigmentosa in India: a genetic and segregation analysis. Clin Genet 1995;47:75-9.

37. Chakrabarti S, Kaur V, Singh D, Ravijit I. Clinical Genetic Analysis of Retinitis Pigmentosa in Indian Population. Indian J Hum Gent 2001;1:133-7.

38. Singh HP, Jalali S, Narayanan R, Kannabiran C. Genetic Analysis of Indian Families with Autosomal Recessive Retinitis Pigmentosa by Homozygosity Screening. Invest Ophthalmol Vis Sci 2009;18:1199-216.

39. Koenekoop RK. An overview of Leber congenital amaurosis: a model to understand human retinal development. Surv Ophthalmol 2004;49:379-98.

40. Ramprasad VL, Soumittra N, Nancarrow D et al. Identification of a novel splice-site mutation in the Lebercilin (LCA5) gene causing Leber congenital amaurosis. Mol Vis 2008; 14:481-6. 
41. Sundaresan $P$, Vijayalashmi $P$, Thompson $S, K o$ AC, Fingert JH, Stone EM. Mutations that are a common cause of Leber congenital amaurosis in northern America are rare in southern India. Mol Vis. 2009;4:1781-7.

42. Verma A, Perumalsamy V, Shetty S, Kulm M, Sundaresan P. Mutational Screening of LCA Genes Emphasizing RPE65 in South Indian Cohort of Patients. PLoS One 2013;8:e73172.

43. Srilekha S, Arokiasamy T, Srikrupa NN et al. Homozygosity Mapping in Leber Congenital Amaurosis and Autosomal Recessive Retinitis Pigmentosa in South Indian Families. PLoS One 2015;10:e0131679. 



\section{ADAPTIVE OPTICS IMAGING OF THE RETINA}

Rajani Battu, Supriya Dabir, Anjani Khanna, Anupama Kiran Kumar, Abhijit Sinha Roy INDIAN JOURNAL OF OPHTHALMOLOGY 2014;62(1):60-5. 



\section{Abstract}

Adaptive optics is a relatively new tool that is available to ophthalmologists for study of cellular level details. In addition to the axial resolution provided by the spectral-domain optical coherence tomography, adaptive optics provides an excellent lateral resolution, enabling visualization of the photoreceptors, blood vessels and details of the optic nerve head. We attempt a mini review of the current role of adaptive optics in retinal imaging in normal subjects, myopes and inherited retinal diseases.

PubMed search was performed with key words Adaptive optics OR Retina OR Retinal imaging. Conference abstracts were searched from the Association for Research in Vision and Ophthalmology (ARVO) and American Academy of Ophthalmology (AAO) meetings. In total, 261 relevant publications and 389 conference abstracts were identified. 


\section{Introduction}

The invention of the direct ophthalmoscope gave a boost to the study of retinal diseases, with the knowledge of reversibility of rays and paraxial viewing. A further development was the indirect ophthalmoscope, which allowed rays to form real images using the same system that converged the light into the eye. Unlike imaging of everyday objects, imaging of the retina is limited by the size of the entrance pupil, and the outgoing rays being imaged are restricted by the size of the same exit pupil. ${ }^{1,2}$

The ability to retrieve these outgoing rays and form an image was possible with the development of the fundus camera. Although limited initially by the film, its resolution and image characteristics were significantly enhanced with charge-coupled devices (CCD cameras) producing a digital image. ${ }^{3}$

The Scanning Laser Ophthalmoscopy (SLO) marked a change in photographic capture technique which utilized the principle of illuminating only a small spot of the retina with collimated laser light directed through the very centre of the pupil and capturing the reflections from its entire surrounds within the pupil confines. ${ }^{4}$ This reversed the optics of entrance and exit imaging and allowed usage of much lower levels of light energy with better images. However, the small spot imaged at each instant of time necessitated a scanning system to allow a large area of the fundus to be scanned and produce an image after collating the scans with appropriate software; this was done with a series of rotating polygonal mirrors and galvanometric mirrors to produce a 'raster scan'. This resulted in two effects on the viewing of the image. Axial resolution was further addressed by using a simple, but ingenious principle of "confocal scanning" where a pinhole of a sufficient diameter interposed before the photodetector system allowed scattered light in front of and behind "the plane of focus" to be reflected off and only the 'confocal rays' finding their way through the pinhole and onto the photodetector. This allowed micrometer precision axial resolution at an image 'plane' combined with the lateral resolution offered by the SLO.

Ocular Coherence tomography (OCT) added a new dimension to retinal imaging with the ability to develop cross sectional images of retinal tissue to a submicron axial resolution and millimeter penetration using a low coherence interference technique combined with a broadband light source. ${ }^{5}$ Time domain initially and later Fourier domain OCT techniques have further improved axial resolution of tissue cross-section. 
Despite technological advancements, imaging of the retina was limited by the 'optical aberrations' of the eye, also expressed as 'wave aberrations'. Wavefront distortions of incoming light limit the eye's cones' resolution capacity. Conversely, wave front distortions of light emerging from the eye, limit the capacity of optical systems' lateral resolution.

Wavefront aberrations may be Low order (LOA - defocus or astigmatism) or High order (HOA-coma, trefoil and spherical aberration). ${ }^{6}$ The LOA aberrations constitute about $90 \%$ of all aberrations of the human eye and have been addressed effectively in many optical imaging devices including the fundus camera. However, HOAs still pose a limit to the lateral resolution capability, as they are nonstable; recognition and correction have to be continuous to allow for excellent resolution.

Adaptive optics (AO) technology aims to correct these ocular aberrations and enhance performance of the optical systems. AO was first used in astronomical telescopes to allow for correction of atmospheric aberrations on wavefronts entering telescopes leading to significant loss of resolution and contrast. ${ }^{7}$

The three principal components of such an AO system include a wavefront sensor (typically Hartman-Shack), a deformable mirror and software to control the interaction between these two components. The wavefront sensor measures the native aberrations of the eye in vivo. The deformable mirror uses a complex system of actuators to adjust several small mirrors to compensate for the aberrations measured. The interaction between the two is controlled by specialized software (Figure 1).6,8

\section{FIGURE 1.}

Schematic outline of adaptive optics imaging system. A beam of light enters the eye, and a small amount is reflected back out of the eye and into the optical system. Reflected light is altered by the deformable mirror for optical aberrations based on measurements made by the wave-front senor. Information about the aberrations of the wave front is processed by the control system that provides feedback to the deformable mirror

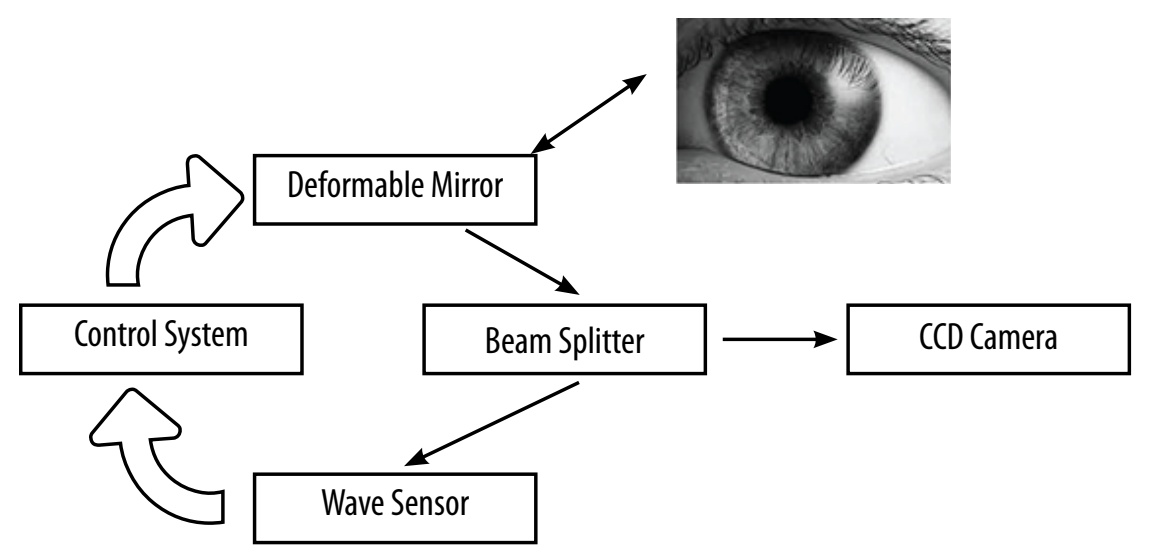


Adaptive optics in retinal imaging could be used with flood illumination or combined with both the SLO and the OCT to produce high quality images whose lateral resolution permits cone discrimination and also allows the study of blood vessels and cells. ${ }^{9,}{ }^{10}$ By compensating for the aberrations caused by irregularities of the optics of the eye, lateral (transverse) resolutions to the order of $2 \mu \mathrm{m}$ can be achieved, thereby allowing for the visualization of individual cone photoreceptors. ${ }^{11}$

With the rapidly increasing potential for adaptive optics imaging, we herein provide a mini-review of the current status of $A O$ in retinal imaging.

\section{Applications}

AO system allows direct visualization of individual rod and cone photoreceptor cells, retinal pigment epithelial (RPE) cells, red and white blood cells, lamina cribrosa and retinal blood vessels. Cone photoreceptors are easily imaged owing to their unique wave-guiding properties, and most of the research published to date is focused on this. ${ }^{12-16}$ Imaging rods is more tedious given their small diameter $(\sim 2 \mu \mathrm{m})$, and their significantly reduced wave-guiding properties compared to cones. ${ }^{17}$

\section{AO Imaging in Normal Subjects}

Data from the AO imaged in healthy eyes is necessary in establishing a baseline of the cone characteristics in vivo, to enable detection of retinal diseases at a very early stage.

One of the AO prototypes (rtx1, Imagine Eyes, Orsay, France) operates at a wavelength of $750 \mathrm{~nm}$ to measure and correct aberrations while maintaining focus at different retinal layers. Another light source of $850 \mathrm{~nm}$ is used to illuminate the retina and capture an image of $40 \times 40$. For fixation, the device has a yellow cross, which can be moved by the investigator. A sequence of 40 frames (frame rate, $9.5 \mathrm{fps}$ ) is acquired on overlapping regions of the posterior pole (Figure 2) 
FIGURE 2.

Raw image before analysis from the rtx 1 (from a normal subject). Note the multiple white dots corresponding to the cones

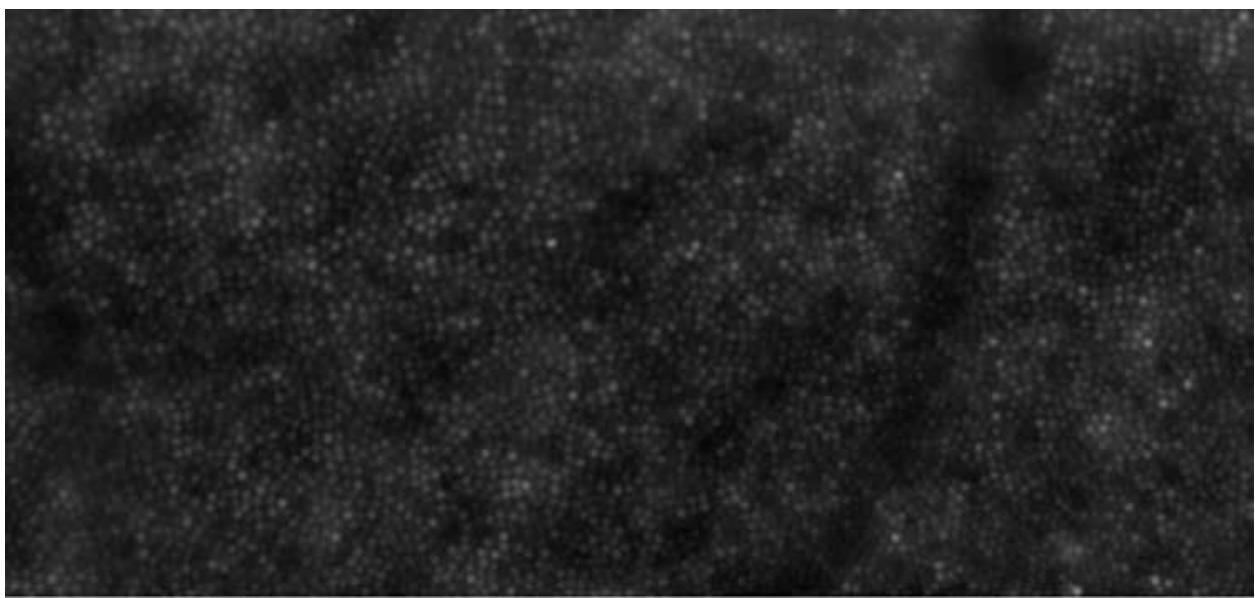

After acquisition, a program provided by the manufacturer is used to produce a final image with enhanced signal-to-noise ratio. Frames exhibiting large motion artifacts owing to eye movement or blinking are manually removed before processing. Before analysis, the retinal magnification factor is calculated in each eye using the Gullstrand schematic eye as a model.

Image analysis of the acquired image is then performed using Image J (version 1.45a, $\mathrm{NIH}$, Bethesda, MD). Once the region of interest (ROI) is selected, analysis of the region provides three parameters mainly - cone packing density, cone spacing and the Voronoi analysis. The Voronoi analysis assesses the regularity of the photoreceptors and the percentage of hexagonal polygons. The ROI can be selected as specific areas in the posterior pole, for comparison with various structural pathologies. The possible range of spherical correction on the rtx 1 is $-12 D S$ to $+6 D S$. Some AO systems are unable to resolve the central foveal cones due to the high foveal cone packing density (Figures 3-5). 
FIGURE 3.

Cone packing density in a normal subject

on the rtx 1 . The encircled area shows

the mean and Standard Deviation (SD)

of cone density and cone spacing in the

central 40X40

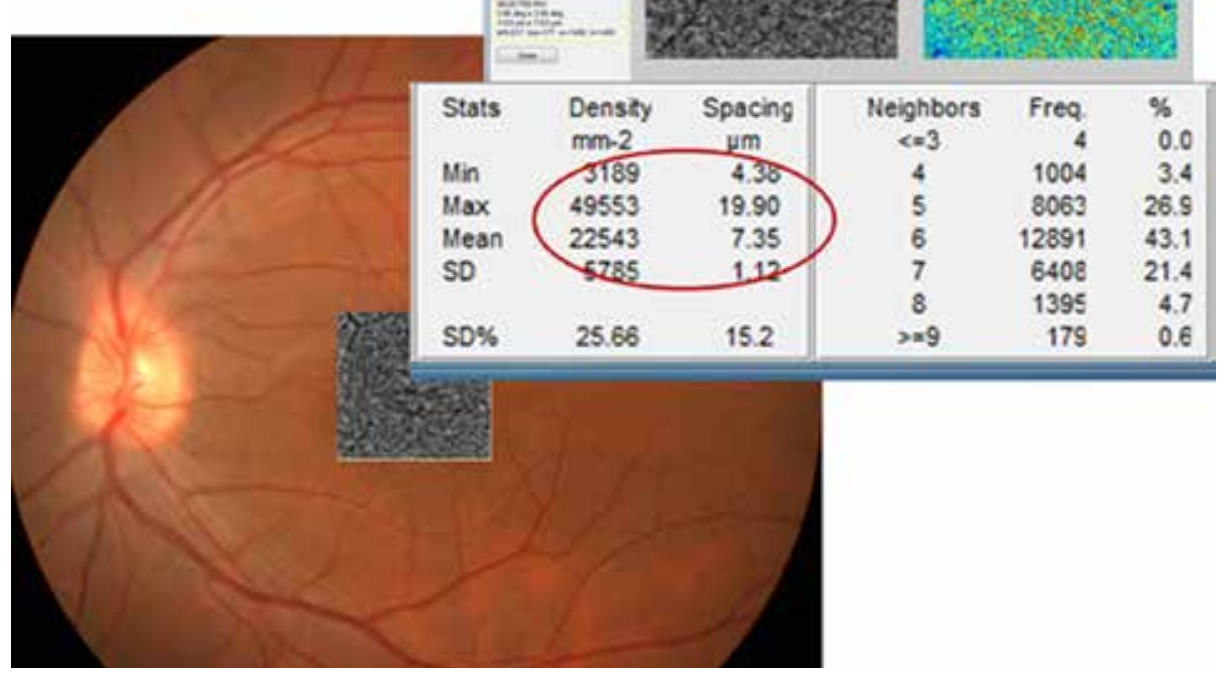

FIGURE 4.

Voronoi tessellation of photoreceptors (cones) in a normal subject on the rtx 1. The colour scale shows the packing density in the central $40 \times 40$. The apparent low cone density depicted in the foveal centre is due to a lack of adequate resolution of the device in that region. In this patient, the mean cone count was $17428 \pm 6743 / \mathrm{mm} 2$
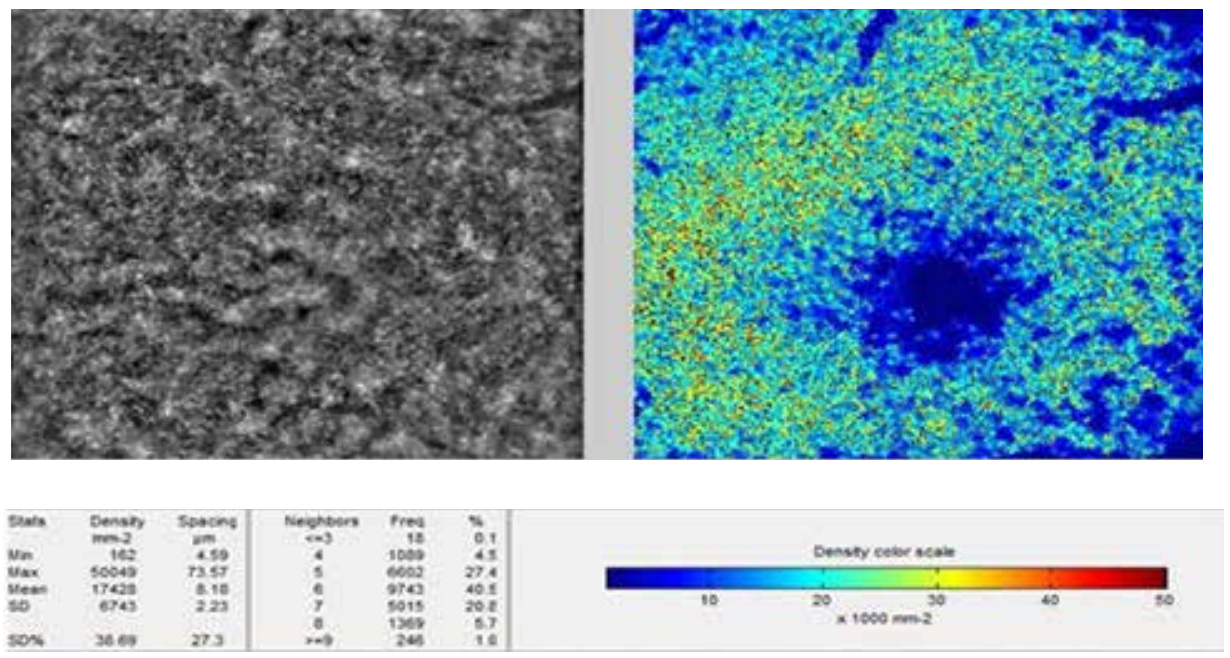
FIGURE 5.

Voronoi tessellation of cones in a $40 \times 40$ square of a normal subject. The color scale shows the Voronoi legend.
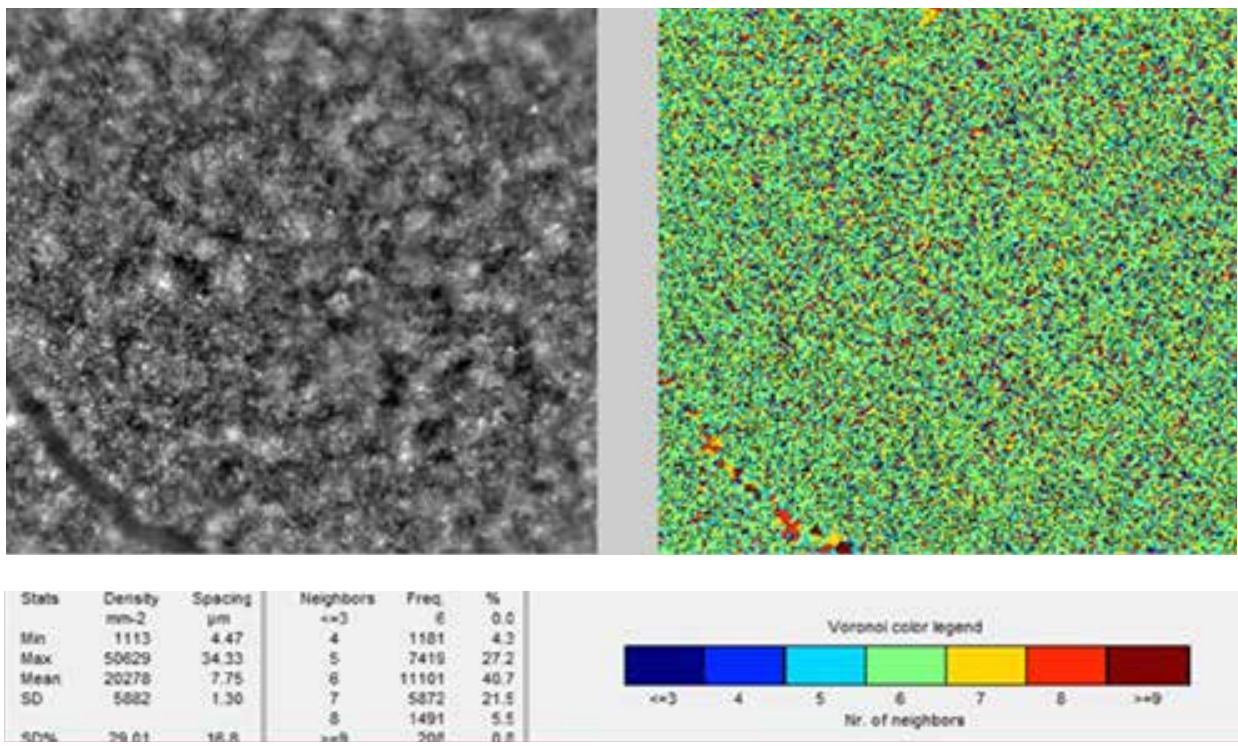

In a study by Chui et al. cone densities ranged from approximately 40,000 cones $/ \mathrm{mm} 2$ at $1^{0}$ to 10,000 cones $/ \mathrm{mm}^{2}$ at $7^{0}$ from the fovea. They also indicated that the human cone photoreceptors are arranged hexagonally near the fovea with the cones becoming more irregular as the retinal eccentricity increases, perhaps due to rod intrusion. ${ }^{18} \mathrm{~A}$ study by Park et al. using a Canon prototype AO-SLO showed a cone density of 32,200 cells $/ \mathrm{mm}^{2}$ at $0.5 \mathrm{~mm}$ from the fovea, which decreased to 11,600 cells $/ \mathrm{mm} 2$ at $1.5 \mathrm{~mm}$ from the fovea. ${ }^{19}$ They did not find demographic and clinical factors, such as gender, ocular dominance, and race/ethnicity to be determinants of cone packing density. Only retinal eccentricity and axial length were significantly associated with measured cone packing density.

Lombardo $\mathrm{M}$ et al. using the $\mathrm{rtx} 1 \mathrm{AO}$ observed that with the exception of the central fovea $(<160 \mu)$, the photoreceptor structure was well resolved in most of the eyes. The inter-individual variation in cone density was $16 \%$, between $260 \mu \mathrm{m}$ and $600 \mu \mathrm{m}$ eccentricity from the foveal center. ${ }^{8}$ Song et al. reported that cone-packing density in the living human retina decreased as a function of age. ${ }^{20}$ 


\section{AO in Myopia}

Axial length $(A L)$ measurements are an important consideration in the analysis of cone packing density. Although the mechanisms are not completely understood, it has been shown that the eye continues to grow into early adulthood, while the cones reach their final number early in development. ${ }^{21}$ It therefore stands to reason that cone density varies across eyes with varying axial lengths.

In a population of 18 eyes of 18 healthy subjects (age range 23 to 43 years; AL range 22.86 to $28.31 \mathrm{~mm}$ ), Li et al. found that cone density decreased significantly with increasing $A L$ at eccentricities between $100 \mathrm{~mm}$ and $300 \mathrm{~mm}$ from the foveal center. ${ }^{22}$

Chui et al. reported cone packing density variations with refractive error; they found that the cone packing density was lower in highly myopic eyes than in emmetropic eyes at both $1.0 \mathrm{~mm}$ and $2.0 \mathrm{~mm}$ eccentricity from the foveal center. ${ }^{18}$ They also compared differences between eyes based on simple angular density of the conesthat is, using the visual angle rather than retinal size, which required correcting the data for the axial length of the eye. They found that the cone packing density was much more constant with axial length when expressed in cells per square degree of visual subtense than when expressed as cells per $\mathrm{mm} 2$ of retinal surface area. ${ }^{18}$

Park et al. also found a statistically significant negative correlation between axial length and cone packing density (decrease of 341 cell $/ \mathrm{mm}^{2}$ per $1 \mathrm{~mm}$ ) increase in axial length. ${ }^{19}$ Lombardo et al. also detected a higher amount of intraocular higher order aberrations in myopic eyes. The retinal stretching resulting from the increased $A L$ in the eye has been postulated to cause the lower cone density estimated in myopic eyes. ${ }^{18}$

\section{Inherited Retinal Diseases}

Inherited retinal degenerations affect about 1:2000 to 1:7000 people worldwide. ${ }^{23}$ These are a group of disorders like Retinitis Pigmentosa (RP), Stargardt disease, Usher's syndrome and Cone-rod dystrophies that are characterized by slow progressive death of rod and cone photoreceptors and relentless vision loss. ${ }^{24}$ There is presently no definitive treatment for most of these; several therapeutic trials that are underway include stem cells, gene therapy, neuroprotective drugs and retinal prosthesis. ${ }^{25-27}$ One limitation to the development of these modalities has been lack of sensitive outcome measures of disease progression and response to therapy. Tests of visual 
function cannot be used as outcome measures as significant photoreceptor loss must have occurred before reliable significant differences are measurable in visual function. Objective sensitive measures of photoreceptor survival may reduce the time to identify a treatment effect of an experimental therapy; studying surviving photoreceptors using the adaptive optics may be one step in this direction. It is therefore not surprising that the majority of scientific work on the AO is dedicated to the study of photoreceptors, mostly cones (Figure 6).

\section{FIGURE 6.}

Voronoi tessellation of photoreceptors (cones) in a patient with rod-cone dystrophy on the rtx 1 . The colour scale shows the packing density in the superior $100 \mu \times 100 \mu\left(0.34^{\circ} \times 0.34^{\circ}\right)$. In this patient, the mean cone count was 2683 $\pm 4006 / \mathrm{mm} 2$

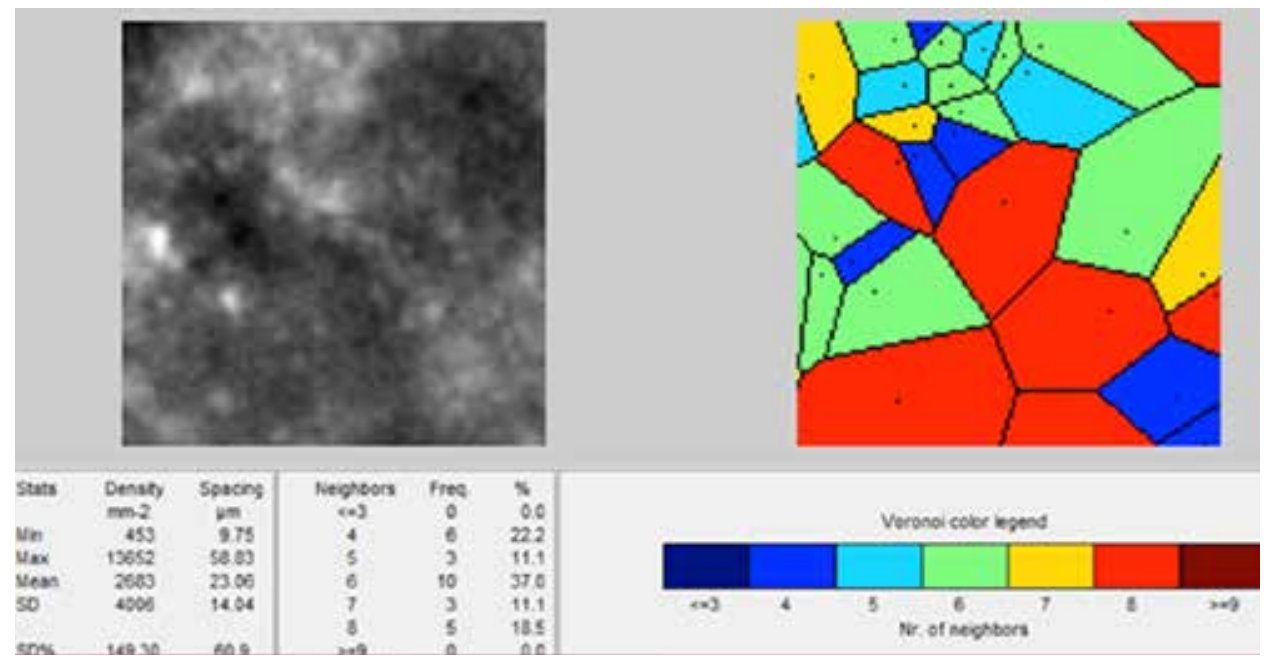

AO has been used in the following instances in the study of photoreceptors

1. To study photoreceptor density and structure in patients with inherited retinal degenerations:

Various groups have studied the cone photoreceptor structure and their functional correlation in inherited retinal disorders. Chen etal. used AO-SLO,SD-OCT and fundus guided microperimetry to study macular cone structure, lipofuscin deposition and visual function in patients with Stargardt disease and found a correlation with abnormal autofluorescence and abnormalities of cone morphology and packing on $\mathrm{AO}$ with corresponding impaired function. ${ }^{28}$ 
Tojo et al. found a correlation between AO images, OCT and fundus autofluorescence (FAF) images in 2 patients with RP. They demonstrated that the edge of the high density FAF ring (that corresponded to the border of external limiting membrane and inner segment- outer segment (IS-OS) line on OCT) showed blurring of cone photoreceptors at the ring as compared to controls on AO. They attributed this to loss of photoreceptor outer segments in patients with $\mathrm{RP}^{29}$

Other studies have demonstrated the correlation of cone parameters on $A O$ images with measures of central visual function including visual acuity, foveal threshold and multifocal electroretinogram (ERG) in patients with RP and conerod dysfunction. Cone spacing measures were also seen to be reproducible suggesting that these can be useful in monitoring disease progression and response to treatment. ${ }^{30,31}$

Rod photoreceptors have also recently been imaged with AO-SLO and the emergence of first rods has been demonstrated at $190 \mu$ from foveal centre as demonstrated by histopathological studies. ${ }^{32}$ The rod mosaic best focus has been seen to be located $10 \mu$ shallower than cones at retinal eccentricities $>8^{\circ}$. This can open new insights into the study of rod disorders.

2. Assessment of photoreceptor function:

Variations in photoreceptor reflectivity have been observed in different disease states. Photoreceptor reflectivity in $\mathrm{AO}$ appears to represent an optical biomarker of photoreceptor integrity. In patients with achromatopsia the remaining cones are sparse and have been shown to reduce reflectivity. Similar cone phenotype is also seen in patients with opsin mutations, acute macular neuroretinopathy $(\mathrm{AMN})$ and closed globe blunt ocular trauma. ${ }^{33}$

Rod and cone photoreceptors have been also shown to vary in intensity over time and by developing methods to quantify this temporal variability more insight into the health of photoreceptors may be available. ${ }^{34}$

3. To decide which patients will benefit the most from treatment:

AO imaging of achromatopsia has revealed varying degrees of retained cone structure. Studying the structure and function of retained cones can set a baseline to assess those patients who may benefit from gene therapy (as has been successful in dog and mouse models) and to anticipate the degree of functional improvement that can be expected based on the patient's baseline 
cone count. ${ }^{14}$ Hence it can help prioritize those patients who can undergo trial of gene therapy (i.e those with certain number of retained cones) over patients who may not benefit from therapy (those with absent cones).

4. Monitoring response to treatment:

In a study by Talcott et al. AOSLO was used to longitudinally study the cone spacing and density in 3 patients implanted with Ciliary Neurotrophic Factor (CNTF) encapsulated implant. ${ }^{35}$ While other modalities like visual field sensitivity and electroretinography responses did not show significant changes at 24 months, AOSLO images showed significantly reduced rates of cone loss in CNTF treated eyes. Longitudinal imaging and monitoring of individual cones was also done and they concluded that AOSLO provided a sensitive measure of disease progression and treatment responses in patients with retinal degenerations.

\section{Vascular Analysis on $\mathrm{AO}$}

Imaging and analysis of the retinal vasculature can be a surrogate marker for systemic vascular health. Correlations between vascular abnormalities in the retina and elsewhere in the body have been demonstrated in a diverse array of conditions including diabetes, hypertension, stroke and migraine. The earliest change in disease begins at the level of the microvasculature; however, current imaging methods lack the lateral resolution to track blood flow at the capillary level. ${ }^{36}$ Imaging retinal capillaries is difficult because of their small size, low contrast, and arrangement in multiple planes of varying retinal depth.

Due to its high-magnification, resolution, and real time visualization it is possible to observe details of vascular wall and contents using AO (Figure 7). Lombardo et al. used an AO flood-illumination retinal camera and described a characteristic pattern of the lumen of a blood vessel, consisting of a central high-intensity channel and two peripheral darker channels. They attributed this difference in intensity to the curvature of the vessel wall and the different shear rate of erythrocytes. ${ }^{37}$ In a subset of diabetics, they showed the capillary lumen caliber to be significantly narrower in NPDR eyes than age matched controls. ${ }^{34}$ 
FIGURE 7.

High resolution image of the venule and arteriole. The black arrow points to the vessel wall (venule). Note the defocused retinal nerve fibre layer in the background

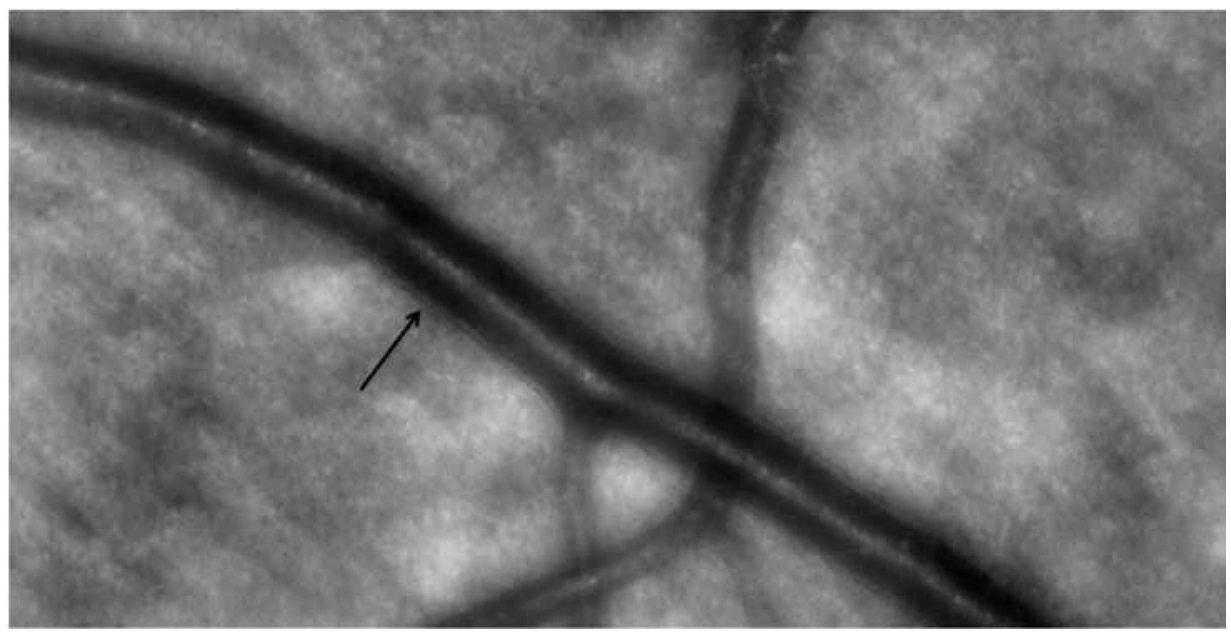

Martin and Roorda imaged parafoveal capillary leukocyte movement and measured their velocity without contrast dyes. A follow up study investigated the possible role of the cardiac cycle on capillary leukocyte velocity by directly measuring capillary leukocyte pulsatility. ${ }^{38}$

The detection of preclinical abnormalities of retinal microcirculation may potentially represent an additional advantage of $\mathrm{AO}$ retinal imaging in patients with diabetes and cardiovascular diseases. Tam et al. evaluated the parafoveal capillary network in patients with Type 2 diabetes. They showed a capillary dropout and a higher tortuosity of the arteriovenous channels in patients with no retinopathy compared to healthy controls. ${ }^{39}$ Popovic $Z$ et al. imaged the foveal capillary networks of five healthy subjects with no previous history of ocular or neurologic disease or surgery with a novel high-resolution, wide-field dual-conjugate adaptive optics (DCAO) imaging instrument. ${ }^{39}$ Their results showed a mean foveal avascular zone (FAZ) area of $0.302 \pm$ 0.100 sqmm and mean equivalent diameter of $612 \pm 106 \mu \mathrm{m} .{ }^{40}$

\section{Clinical Limitations and Future Prospects of Adaptive Optics}

As adaptive optics imaging finds more clinical applications, normative databases need to be developed for different populations as a reference for disease states. Universally 
acceptable standards and protocols need to be defined for image acquisition and analysis to ensure reproducibility and comparability worldwide. Acquisition of $A O$ images of sufficient quality to perform quantitative cone spacing measures is difficult in patients with unstable fixation, dense cataracts and other media opacities. The use of AO may be limited owing to costs and lack of expertise.

In summary, AO is an innovative new tool in the extensive armamentarium of ophthalmologists to explore the cellular details of the retina. It may help us understand the pathogenesis of diseases at a subclinical stage, thus paving way for the exciting possibility of early treatment for these diseases. It is possible that as more detailing of cellular structures becomes possible, we may need to develop better analytical tools. As treatment paradigms for inherited retinal diseases evolve, it is likely that AO systems will play a major role in early diagnosis and monitoring therapies.

\section{References}

1. Colenbrander A. Principles of ophthalmoscopy. In: Tasman W, Jaeger EA, eds Duane's Ophthalmology 2013 ed 2012. , vol 1, chap 63.

2. Miller D TE, Atebara NH. Ophthalmic instrumentation. In: Yanoff M, Duker JS, eds Ophthalmology 2008;chap 2.10.

3. Meyer-Schwickerath GR. Ophthalmology and photography. Am J Ophthalmol 1968;66:1011-9.

4. Mainster MA, Timberlake GT, Webb RH, Hughes GW. Scanning laser ophthalmoscopy. Clinical applications. Ophthalmology 1982;89:852-7.

5. Puliafito CA, Hee MR, Lin CP, et al. Imaging of macular diseases with optical coherence tomography. Ophthalmology 1995;102:217-29.

6. Lombardo M, Lombardo G. Wave aberration of human eyes and new descriptors of image optical quality and visual performance. J Cataract Refract Surg 2010;36:313-31.

7. Babcock HW. Adaptive optics revisited. Science 1990;249:253-7.

8. Lombardo M, Serrao S, Ducoli P, Lombardo G. Eccentricity dependent changes of density, spacing and packing arrangement of parafoveal cones. Ophthalmic Physiol Opt 2013;33:516-26.

9. Duncan JL, Zhang $Y$, Gandhi J, et al. High-resolution imaging with adaptive optics in patients with inherited retinal degeneration. Invest Ophthalmol Vis Sci 2007;48:3283-91. 
10. Torti $C$, Povazay $B$, Hofer $B$, et al. Adaptive optics optical coherence tomography at 120,000 depth scans/s for non-invasive cellular phenotyping of the living human retina. Opt Express 2009;17:19382-400.

11. Zhang $Y$, Roorda A. Evaluating the lateral resolution of the adaptive optics scanning laser ophthalmoscope. J Biomed Opt 2006;11:014002.

12. Godara P, Dubis AM, Roorda A, Duncan JL, Carroll J. Adaptive optics retinal imaging: emerging clinical applications. Optom Vis Sci 2010;87:930-41.

13. Lombardo M, Lombardo G, Ducoli P, Serrao S. Adaptive optics photoreceptor imaging. Ophthalmology 2012;119:1498-e2.

14. Carroll J, Kay DB, Scoles D, Dubra A, Lombardo M. Adaptive optics retinal imaging-clinical opportunities and challenges. Curr Eye Res 2013;38:709-21.

15. Kim JE, Chung M. Adaptive optics for retinal imaging: current status. Retina 2013;33:1483-6.

16. Lombardo M, Serrao S, Devaney N, Parravano M, Lombardo G. Adaptive optics technology for high-resolution retinal imaging. Sensors (Basel) 2013;13:334-66.

17. Alpern M, Ching CC, Kitahara K. The directional sensitivity of retinal rods. J Physiol 1983;343:577-92.

18. Chui $T Y$, Song H, Burns SA. Adaptive-optics imaging of human cone photoreceptor distribution.J Opt Soc Am A Opt Image Sci Vis 2008;25:3021-9.

19. Park SP, Chung JK, Greenstein V, Tsang SH, Chang S. A study of factors affecting the human cone photoreceptor density measured by adaptive optics scanning laser ophthalmoscope. Exp Eye Res 2013;108:1-9.

20. Song H, Chui TY, Zhong Z, Elsner AE, Burns SA. Variation of cone photoreceptor packing density with retinal eccentricity and age. Invest Ophthalmol Vis Sci 2011;52:7376-84.

21. Grosvenor T, Scott R. Role of the axial length/corneal radius ratio in determining the refractive state of the eye. Optom Vis Sci 1994;71:573-9.

22. Li KY, Tiruveedhula P, Roorda A. Intersubject variability of foveal cone photoreceptor density in relation to eye length. Invest Ophthalmol Vis Sci 2010;51:6858-67.

23. Fasiuddin A. Inherited retinal degenerations. Int Ophthalmol Clin 2010;50:45-56.

24. Hartong DT, Berson EL, Dryja TP. Retinitis pigmentosa. Lancet 2006;368:1795-809.

25. Stone EM. Progress toward effective treatments for human photoreceptor degenerations. Curr Opin Genet Dev 2009;19:283-9.

26. Huang Y, Enzmann V, Ildstad ST. Stem cell-based therapeutic applications in retinal degenerative diseases. Stem Cell Rev 2011;7:434-45. 
27. Farrar GJ, Millington-Ward S, Chadderton N, Humphries P, Kenna PF. Gene-based therapies for dominantly inherited retinopathies. Gene Ther 2012;19:137-44.

28. Chen Y, Ratnam K, Sundquist SM, et al. Cone photoreceptor abnormalities correlate with vision loss in patients with Stargardt disease. Invest Ophthalmol Vis Sci 2011;52:3281-92.

29. Tojo N, Nakamura T, Fuchizawa C, Oiwake T, Hayashi A. Adaptive optics fundus images of cone photoreceptors in the macula of patients with retinitis pigmentosa. Clin Ophthalmol 2013;7:203-10.

30. Wolfing Jl, Chung M, Carroll J, Roorda A, Williams DR. High-resolution retinal imaging of cone-rod dystrophy. Ophthalmology 2006;113:1019e1.

31. Choi SS, Doble N, Hardy JL, et al. In vivo imaging of the photoreceptor mosaic in retinal dystrophies and correlations with visual function. Invest Ophthalmol Vis Sci 2006;47:2080-92.

32. Dubra A, Sulai Y, Norris JL, et al. Noninvasive imaging of the human rod photoreceptor mosaic using a confocal adaptive optics scanning ophthalmoscope. Biomed Opt Express 2011;2:1864-76.

33. Genead MA, Fishman GA, Rha J, et al. Photoreceptor structure and function in patients with congenital achromatopsia. Invest Ophthalmol Vis Sci 2011;52:7298-308.

34. Cooper RF, Dubis AM, Pavaskar A, Rha J, Dubra A, Carroll J. Spatial and temporal variation of rod photoreceptor reflectance in the human retina. Biomed Opt Express 2011;2:2577-89.

35. Talcott KE, Ratnam K, Sundquist SM, et al. Longitudinal study of cone photoreceptors during retinal degeneration and in response to ciliary neurotrophic factor treatment. Invest Ophthalmol Vis Sci 2011;52:2219-26.

36. Bedggood P, Metha A. Direct visualization and characterization of erythrocyte flow in human retinal capillaries. Biomed Opt Express 2012;3:3264-77.

37. Lombardo M, Parravano M, Serrao S, Ducoli P, Stirpe M, Lombardo G. Analysis of retinal capillaries in patients with type 1 diabetes and nonproliferative diabetic retinopathy using adaptive optics imaging. Retina 2013;33:1630-9.

38. Martin JA, Roorda A. Direct and noninvasive assessment of parafoveal capillary leukocyte velocity. Ophthalmology 2005;112:2219-24.

39. Tam J, Martin JA, Roorda A. Noninvasive visualization and analysis of parafoveal capillaries in humans. Invest Ophthalmol Vis Sci 2010;51:1691-8.

40. Popovic $Z$, Knutsson P, Thaung J, Owner-Petersen $M$, Sjostrand J. Noninvasive imaging of human foveal capillary network using dual-conjugate adaptive optics. Invest Ophthalmol Vis Sci 2011;52:2649-55. 



\section{CHAPTER 4}

\section{ADAPTIVE OPTICS IMAGING OF THE OUTER RETINAL TUBULES IN BIETTI'S CRYSTALLINE DYSTROPHY}

Rajani Battu, Mukunda C Akkalli, Devanshi Bhanushali, Priya Srinivasan, Rohit Shetty, Tos TJM Berendschot, Jan SAG Schouten, Carroll AB Webers

EYE (LOND) 2016: 30(5); 705-12 



\section{Abstract}

PURPOSE: To study the outer retinal tubules using Spectral domain optical coherence tomography and Adaptive optics and in patients with Bietti's crystalline Dystrophy.

METHODS: Ten eyes of five subjects from five independent families with Bietti's crystalline Dystrophy (BCD) were characterized with best-corrected visual acuity (BCVA), full-field electroretinography, and fundus autofluorescence (FAF). High-resolution images were obtained with the spectral domain optical coherence tomography (SD$\mathrm{OCT}$ ) and adaptive optics (AO).

RESULTS: SD-OCT showed prominent outer retinal layer loss and outer retinal tubulations at the margin of outer retinal loss. AO images displayed prominent macrotubules and microtubules with characteristic features in eight out of the ten eyes. Crystals were present in all ten eyes. There was a reduction in the cone count in all eyes in the area outside the outer retinal tubules (ORT).

CONCLUSIONS: This study describes the morphology of the outer retinal tubules when imaged enface on the adaptive optics in patients with BCD. These findings provide insight into the macular structure of these patients. This may have prognostic implications and refine the study on the pathogenesis of BCD. 


\section{Introduction}

Bietti crystalline dystrophy (OMIM 210370)(BCD) is a rare autosomal recessive disease characterized by the presence of yellow-white crystalline retinal deposits, corneal deposits, progressive atrophy of the retinal pigment epithelium (RPE), and loss of choriocapillaris.' Patients present with progressive night blindness; most of them are legally blind by the fifth to sixth decade of life.' CYP4V2, the gene responsible for $B C D$, codes for a member of the cytochrome $p-450$ family that plays a role in the metabolism of fatty acids. ${ }^{2-6}$

Outer retinal tubulations (ORT) were first described by Zweifel et al.in patients with age-related macular degeneration (AMD). ${ }^{7}$ These appeared as hypo reflective ovoid spaces with hyper reflective borders in the outer nuclear layer on the optical coherence tomography. They have been described in other degenerative retinal diseases like Choroideremia and BCD.,9

New imaging techniques and functional tests have evolved that are used to study patients with retinal dystrophies. These include spectral-domain optical coherence tomography (SD-OCT), fundus autofluorescence (FAF), microperimetry, and adaptive optics (AO) imaging.

AO imaging is a new technology that allows direct visualization of individual rod and cone photoreceptor cells and retinal pigment epithelial (RPE) cells. ${ }^{10}$ Gocho et al. reported an abnormal cone mosaic in the posterior pole of patients with BCD. ${ }^{11}$ There is no detailed description of the ORTs on the AO.

Thus, the purpose of this study was to characterize in detail the outer retinal tubulations seen in $B C D$ using the $A O$ technology. To accomplish this, we studied five patients with $B C D$ using high resolution $S D-O C T$ and $A O$ imaging.

\section{Patients and Methods}

Five patients from five independent families clinically diagnosed with BCD were enrolled into the study; both eyes were included in the study. All 5 patients were isolated cases from non-consanguineous families. All of them were Asian Indian. The study was approved by the institutional ethics committee of Narayana Nethralaya 
and followed the tenets of declaration of Helsinki. Informed consent for the study and publication was obtained from each patient. Every patient underwent a complete ophthalmic examination including assessment of their best-corrected visual acuity (BCVA) with the Snellen's chart, intraocular pressure measurements with the Goldmann applanation tonometer and a detailed fundus evaluation. All patients underwent full-field electroretinography (Veris systems) according to the ISCEV standards. ${ }^{12}$ Both scotopic and photopic responses were recorded. The axial length was measured in all patients using the Lens star system. They underwent further evaluation with Spectral domain optical coherence tomography and Adaptive optics imaging.

\section{SD-OCT I maging}

Imaging was performed using Heidelberg HRA2-OCT (Heidelberg retina angiograph optical coherence tomography, Heidelberg Engineering, Heidelberg, Germany). Each patient underwent a 100 ART (automatic real time) imaging in the vertical and horizontal meridians in addition to the star-scan and volume scan in the central 200. Each image was analyzed for the presence of bright reflective dots and ORTs. Central foveal thickness (CFT) was measured at the centre of the fovea. Using the enhanced depth-imaging mode, sub foveal choroidal thickness (SCT) was measured from the inner border of the sclera to the outer border of the RPE vertically using the calipers of the Heidelberg reader at the same point. ${ }^{13}$

\section{Adaptive Optics Imaging}

Imaging was performed using the flood-illuminated AO retinal camera ( $r$ tx1, Imagine Eyes, Orsay, France). This system has been used in earlier investigations to image individual cone photoreceptors and other retinal structures. ${ }^{14-16}$ The field of imaging was $4^{\circ} \times 4^{\circ}$.

AO imaging sessions were conducted after dilating the pupils with $0.5 \%$ tropicamide and $10 \%$ phenylephrine hydrochloride. Aberrations induced by pupil dilation are negated by the AO system. Stable fixation was maintained by having the patient look at the system's inbuilt target and then as moved by the investigator to pre-determined coordinates. The patient was instructed to fixate at $0^{\circ}, 1^{\circ}, 2^{\circ}$, and $3^{\circ}$ eccentricity along all the four quadrants: superior, inferior, nasal, and temporal retina. A video (a series 
of 40 frames; $4^{\circ}$ field size) was captured at each of the above retinal locations. After the acquisition, a program provided by the manufacturer correlated and averaged the captured image frames to produce a final image with a resolution of $1500 \times 1500$ pixels. $^{17}$

Cone density (cones/mm2) was measured at $1^{\circ}, 2^{\circ}$, and $3^{\circ}$ eccentricity along all the four quadrants: superior, inferior, nasal, and temporal retina. There has been no standardized protocol on which areas to image and on the size of the sampling window to choose the region of interest (ROI). The sampling window we chose was $100 \mu \mathrm{m}$ and we placed it at specific coordinates calculated by a pre-fixed formula intentionally avoiding blood vessels. Our rationale was that the size of the sampling window correlates with the size of the retinal area stimulated by a Goldman size III target, so as to be able to correlate the structure and function in the future. Eccentricity was computed as the distance between the center of each window and the foveal center reference point (identified as the point with fixation coordinates: $x=0^{\circ}, y=0^{\circ}$ ). The images were captured at temporal $\left(-3^{\circ}\right.$ and $\left.0^{\circ}\right)$ superior $\left(0^{\circ}\right.$ and $\left.3^{\circ}\right)$ nasal $\left(3^{\circ}\right.$ and $\left.0^{\circ}\right)$, and inferior $\left(0^{\circ}\right.$ and $\left.-3^{\circ}\right)$. The cone counting software $A O$ detect created on MATLAB by Imagine Eyes was used to process the images and calculate the cone density. Axial length was taken into consideration for calculating cone density and it was measured by non-contact biometry (IOL Master; Carl Zeiss Meditec, Jena, Germany). Both eyes of each patient were imaged.

The resulting images were stitched together using the i2k-retina software from DualAlign's $^{\circledR}$ i2k Retina ${ }^{T M}$. When i2k failed to find the position of an image, the GIMP software was used to stitch the image manually (GNU Image Manipulation Program, The GIMP Development Team; Image J, National Institute of Health, Bethesda, MD).

The diameters of outer retinal tubules (ORT) were measured using an imageprocessing program (Image J, National Institutes of Health, Bethesda, MD, USA). The size of each pixel was typically $0.8 \mu \mathrm{m}$ when calculated at the retinal plane, and the values were adjusted for variations in the axial length of the eye. ${ }^{16,17}$

\section{Statistical Analysis}

The statistical software package IBM SPSS Statistics 20 was used for analysis. All variables investigated exhibited a typical normal distribution. Mean \pm SD's are presented in the text. We used the $5 \%$ level of significance throughout our analysis. 


\section{Results}

There were 3 males and 2 females with mean age of 41.4 years (range 20-60 years). The BCVA on the Snellen's chart ranged from $1 / 60$ to $6 / 6$ (Table 1). Fundus examination in all patients showed intra-retinal, discrete, yellowish white crystals that were concentrated over the macula mainly. Crystals were not visible in the area of RPE atrophy (Figure 1). The older patients (patient 1 and 2) showed extensive atrophy of the choriocapillaris with fewer crystals over the macula while the younger patients (patients 3, 4 and 5) showed more extensive crystals and prominent intra-retinal pigmentation (Figure 1). Retinal vascular attenuation was more prominent in the older patients. Full field ERG was extinguished in three patients, and subnormal in two (Table 1). Four out of the five patients underwent evaluation of the systemic lipid profile; none of them had an abnormal lipid profile.

TABLE 1.

General characteristics of the patients

\begin{tabular}{|c|c|c|c|c|c|c|c|c|c|c|c|c|}
\hline Pt & Age & Sex & & & & & & & $\begin{array}{r}\text { Axial } \\
(n\end{array}$ & $\begin{array}{l}\text { ngth } \\
\text { l) }\end{array}$ & $\begin{array}{c}\text { ERG Phot- } \\
\text { opic }\end{array}$ & ERG scotopic \\
\hline Eye & & & RE & $\mathrm{LE}$ & RE & LE & RE & LE & RE & LE & $\mathrm{BE}$ & BE \\
\hline 1 & 53 & M & $6 / 18, N 12$ & $6 / 24, N 18$ & 0 & 0 & 13 & 12 & 23 & 22.94 & Extinguished & Extinguished \\
\hline 2 & 60 & $M$ & $6 / 18, N 12$ & $6 / 36$, N36 & $\begin{array}{c}0.00 /-0.75 \\
D C 1000\end{array}$ & $\begin{array}{c}+1.0 /-0.25 \\
\text { DC } 900\end{array}$ & 14 & 14 & 23.02 & 23.13 & Extinguished & Extinguished \\
\hline 3 & 32 & $M$ & $\begin{array}{c}\text { 6/24, } \\
\text { N8 }\end{array}$ & $\begin{array}{l}\text { 6/6, } \\
\text { N6 }\end{array}$ & $\begin{array}{c}-5.0 /-0.5 D C \\
400\end{array}$ & $\begin{array}{l}-4.00 \\
\text { DSph }\end{array}$ & 14 & 14 & 25.32 & 25.12 & Subnormal & Subnormal \\
\hline 4 & 42 & $\mathrm{~F}$ & $\begin{array}{l}1 / 60, \\
<N 36\end{array}$ & $6 / 9, N 12$ & 0 & $\begin{array}{l}-4.00 \\
\text { DSph }\end{array}$ & 15 & 17 & 23.22 & 23.06 & Extinguished & Extinguished \\
\hline 5 & 20 & $\mathrm{~F}$ & $\begin{array}{l}1 / 60 \\
<\mathrm{N} 36\end{array}$ & $\begin{array}{l}3 / 60 \\
<\mathrm{N} 36\end{array}$ & 0 & 0 & 12 & 15 & 24 & 24 & Subnormal & Subnormal \\
\hline
\end{tabular}


FIGURE 1.

Fundus pictures of patients. A shows the fundus picture of the left eye of patient 2 ( 60 years old) with prominent atrophy of the retinochoroidal layers, especially over the posterior pole (yellow arrow). Few crystals can be noted outside this area of atrophy (orange arrow). B shows the fundus picture of the left eye of a younger patient 5 (20 years old) who displays prominent crystals over the posterior pole (orange arrow) with no visible areas of choroidal atrophy. C. Fundus autofluorescence (FAF) shows the prominent area of hypoautofluorescence. Note the hyperautofluorescent edge of the lesion (yellow arrow). D. The near-infra red (NIR) image of the same patient displays the retinal crystals prominently (that are not visible on the FAF) (orange arrow)
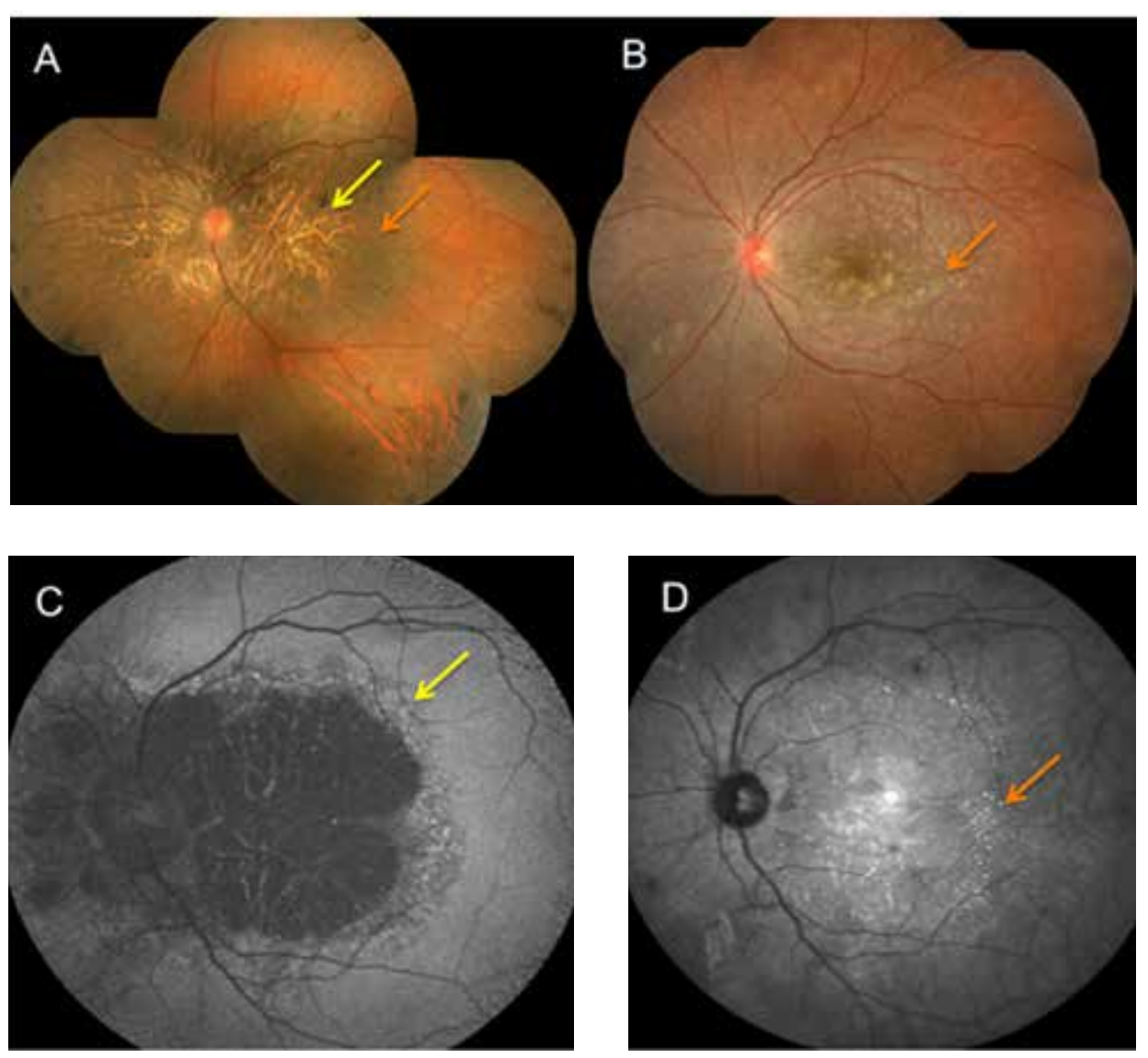


\section{SD - OCT imaging}

FAF showed areas of hypo-autofluorescence (corresponding to RPE atrophy and loss of lipofuscin) with a hyper-autofluorescent edge representing the lipofuscin-laden cells that may atrophy imminently (Figure 1). The crystalline deposits were seen more prominently on the near-infrared (NIR) images (Figure 1). None of our patients had corneal crystals.

SD-OCT in all five patients showed a complete disorganization of the outer retinal layers; the inner retinal architecture was relatively preserved. ORTs were noted in eight eyes in the outer nuclear layer at the junction of the normal and atrophic retina (Figure 2).

The average CFT was $148( \pm 73) \mu \mathrm{m}$. There was considerable foveal thinning in 3 out of the 10 eyes. Marked attenuation of the choroidal layer was noted on the enhanced depth imaging (EDI) scans with an average SCT of $95.9( \pm 25.9) \mu \mathrm{m}$. Patient 5 demonstrated cystoid cavities and ORTs in the peripheral macula (Figure 2).

FIGURE 2.

SD-OCT features of the patients. The pictures on the left panel throughout show the infrared images. A. SD-OCT of the left eye of patient 2 displaying prominent ORTs (yellow arrows). Note that there is a significant disorganization of the outer retina in the regions of the ORTs. Beyond this region, the IS/OS layer is preserved (orange arrow) with a prominent scroll of the ELM at the junction (blue arrow). The inner retinal architecture is relatively preserved. Note the severe choroidal thinning throughout (white arrows). B shows the SD-OCT of patient 1. Note the crystals located on the RPE/Bruch's membrane complex (green arrows). C. SD-OCT of patient 5 showing cystoid macular spaces. The ORTs are visible in the periphery of the section (yellow arrow).
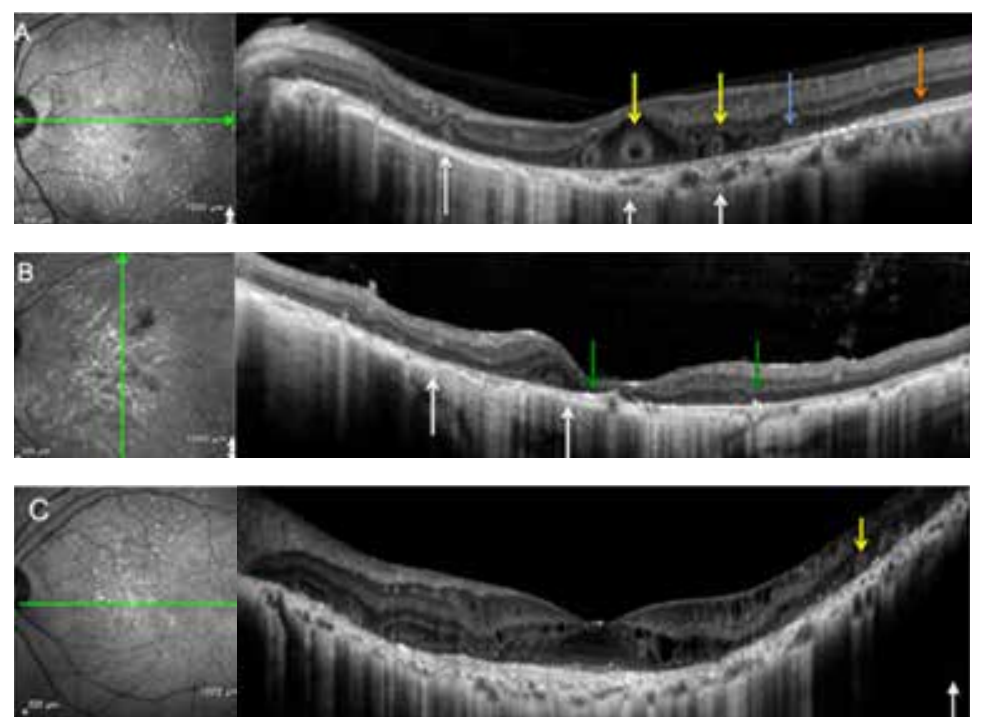


\section{AO Imaging}

Cone count was decreased in all eyes. The average foveal cone count for this group was 7990 cones per $\mathrm{mm}^{2}$ ranging from 1822 to 18023 cones per $\mathrm{mm}^{2}$ area. The average foveal cone count in normal Asian eyes from our institute was 24,000 cones per $\mathrm{mm} 2.18$ The detailed list of cone count including fovea and para fovea are presented in Table 2.

The tubules were imaged enface on the AO. These appeared as elongated serpentine tubules with multiple branching (Figure 3). The tubules varied in size with the smaller ones (referred to as microtubules) showing more extensive and irregular branching with complex networks compared to the macrotubules. These could be clearly distinguished from the overlying blood vessels on the AO. The tubules seem to interconnect and span the entire width of the image taken. The larger tubules seem to branch sparsely compared to the microtubules.

Each macrotubule appeared as alternating dark and light bands spanning the width of each (Figure 3). The wall of the tubule itself appeared relatively thick with a very narrow lumen. Prominent dark patches were visible along the wall of the tubule in its entire course. The alternating bands could not be appreciated in the microtubules. The average size of the microtubules was $47( \pm 19) \mu \mathrm{m}$ and the macrotubules was 412 $( \pm 331) \mu \mathrm{m}$.

The crystals appeared as multiple, isolated hyper-reflective spots distributed irregularly. There was no specific relationship of the crystals to the tubules, although the crystals appeared larger when they were in close proximity to the tubules. The cone count was visibly reduced in the areas of the tubule formation, with very irregular cone mosaic throughout the distribution of the tubules.

In two eyes of patient 5 with extensive crystals, we were not able to identify any tubules on the AO. There were significant cystoid cavities in this patient on the SDOCT (Figure 2). 
FIGURE 3.

Demonstrates the overlay and the $A 0$ image of the ORTs. A. Overlay of the $A 0$ montage on the fundus picture of patient 1. B. Montage of the central foveal and four parafoveal $4 \times 40$ images obtained on the A0. Note the serpentine macro tubules (yellow arrows) that span the entire width of the image and the smaller microtubules (orange arrows) that are more complexly arranged. The blood vessels are denoted by the green arrows. The crystals are denoted by the blue arrows. C. Further magnified view which denotes the macrotubules. Note the branching (red arrow). The tubule is marked by dark bands (white arrows) alternating with light bands throughout its length. Along the wall of the tubule are the dark patches corresponding to areas of RPE atrophy (orange arrows). The crystals themselves appeared larger when present in relation to the tubules (blue arrows) than otherwise. The cone mosaic is greatly reduced especially close to the tubules. D. Note the prominent crystals in the left eye of patient 5.

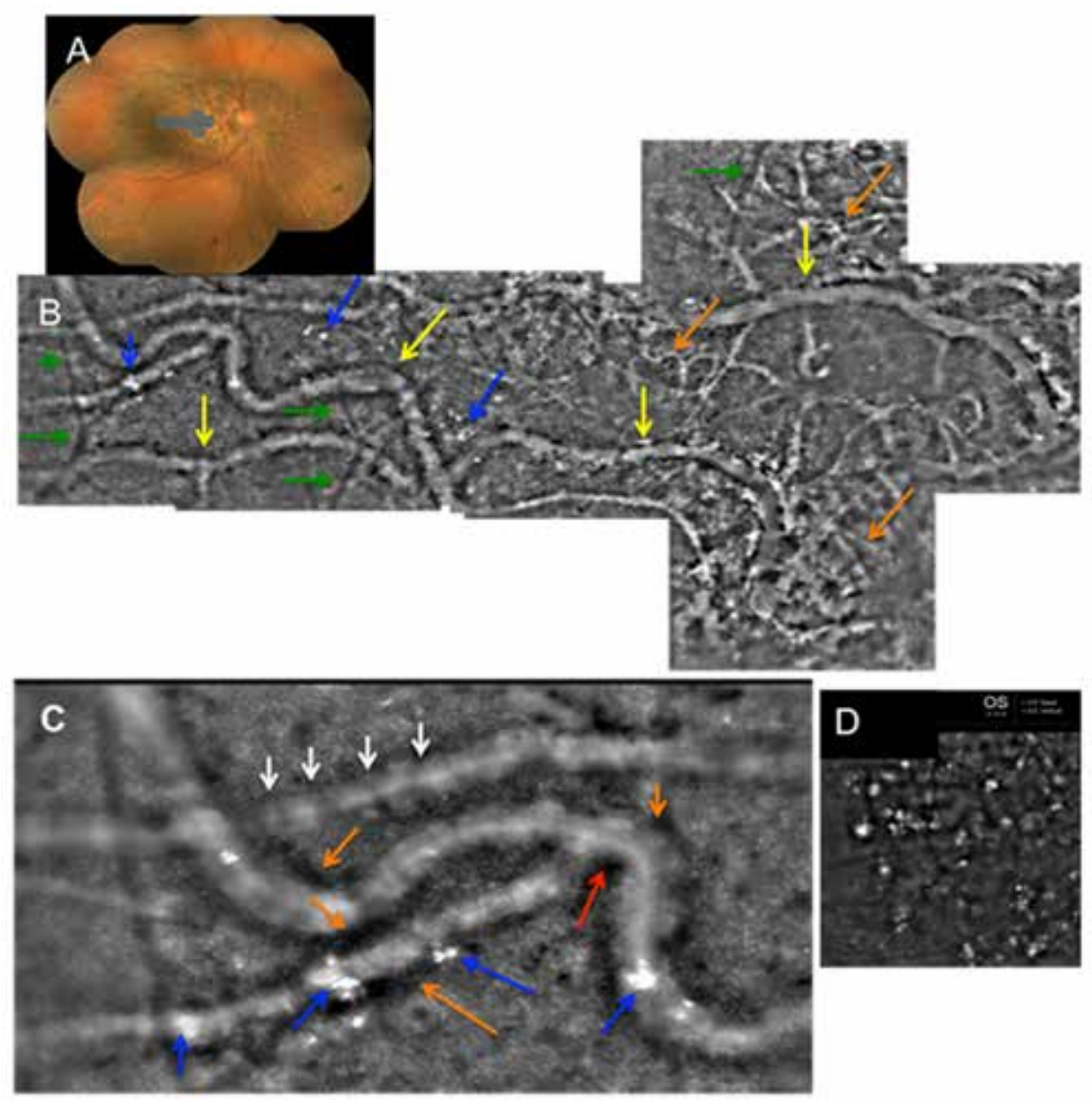


TABLE 2.

Details of macula on the SD-OCT and AO

\begin{tabular}{|c|c|c|c|c|c|c|c|c|}
\hline Patient & \multicolumn{2}{|c|}{$\begin{array}{c}\text { CFT } \\
(\boldsymbol{\mu m})\end{array}$} & \multicolumn{2}{c|}{ SCT $(\boldsymbol{\mu m})$} & \multicolumn{2}{c|}{$\begin{array}{c}\text { Smallest ORT dia } \\
(\boldsymbol{\mu m}) \text { on A0 }\end{array}$} & \multicolumn{2}{|c|}{$\begin{array}{c}\text { Largest ORT dia } \\
(\boldsymbol{\mu m}) \text { on A0 }\end{array}$} \\
\hline Eye & RE & LE & RE & LE & RE & LE & RE & LE \\
\hline 1 & 64 & 43 & 50 & 63 & 33 & 27 & 267 & 295 \\
\hline 2 & 195 & 262 & 114 & 84 & 47 & 35 & 961 & 930 \\
\hline 3 & 123 & 171 & 94 & 80 & 84 & 61 & 193 & 213 \\
\hline 4 & 64 & 171 & 96 & 119 & 32 & 53 & 185 & 253 \\
\hline 5 & 220 & 222 & 127 & 132 & - & - & - & - \\
\hline Mean \pm SD & 133.2 & 173.8 & 96.2 & 95.6 & 49 & 44 & 401.5 & 422.7 \\
& \pm 72.5 & \pm 82.5 & \pm 29.2 & \pm 28.7 & \pm 30.4 & \pm 15.7 & \pm 374 & \pm 339 \\
\hline
\end{tabular}

\section{Discussion}

This study describes the morphology of ORTs on AO imaging. The diagnosis of BCD was made clinically. Genotyping would have been of additional diagnostic value, but we lacked resources to conduct genotyping. Gocho et al. described the crystals on $A O$ imaging in $B C D .{ }^{11}$ Striking in our study was the elongated tubules seen on $A O$, in contrast to the cross section noted on the SD-OCT.

Zweifel et al. proposed that degenerating photoreceptors along with the RPE and glial elements came to be arranged in a tubular fashion, which they called ORTs.7 They believed that these represented the final pathway in many retinal degenerative diseases. Photoreceptor or RPE injury lead to the loss of interdigitations and outward folding of photoreceptors at the junction of intact and disrupted ellipsoid portion of the photoreceptor inner segment initially and formation of a long, ovoid tubular complex eventually. Jung et al. described that an ORT is a rearrangement of the photoreceptor layer in response to injury, in which surviving photoreceptors form new lateral connections with neighboring cells. ${ }^{20}$

Histologically, ORTs were first described as interconnecting tubes containing degenerate photoreceptors and enveloping Müller cells in AMD. ${ }^{21}$ Recently Schaal et al. studied the histology of ORTs in AMD. ${ }^{22}$ They showed that the ORTs were always present in the outer nuclear layer and largely consisted of cones lacking outer segments and cones lacking inner segments. They defined four phases of cone degeneration histologically - nascent, mature, degenerate and end stage ORTs. Unlike 
the hyperreflective border of the ORT seen on the SD-OCT, histologically, the external limiting membrane (ELM) border that included both the inner and the outer segments in the nascent phase and only the inner segments in the other three phases defined them. ${ }^{22}$ The underlying RPE was either dysmorphic or absent. Also, a free edge of the ELM to scroll seemed necessary for its formation. They described that all ORTs did not branch and therefore did not consider branching a defining part of an ORT. With time, they found shrinkage of the inner segments and involution of the ORT. The absence of branching as a consistent finding in their study probably refers to the macrotubules that we noted, since these branched rarely unlike the microtubules that branched more complexly.

Trying to extrapolate the histological findings of Schaal et al.in AMD to the findings we noted on the adaptive optics imaging seems complicated. The alternating dark and light bands seen on the AO possibly correspond to the photoreceptors alternating with Muller cells (and other glial cells). It was hard to distinguish the ELM in the tubules. The cone mosaic seemed better preserved in the In the vicinity of the microtubules compared to that of the macrotubules.

We were unable to distinguish open from closed ORTs on the AO that have been described by Schaal et al. ${ }^{22}$ Histologically ORTs have been shown to involute over time with the inner segments shrinking and the mitochondria migrating through the ELM towards the cone perikaryon. ${ }^{22}$ The conglomeration of multiple microtubules may form the macrotubules. Tubule formation may be an attempt of the photoreceptors to survive in a microenvironment that is doomed to degenerate, by seeking out other photoreceptors and forming long tubules in the process. It is a surprising finding that such well-organized tubules would be part of a degenerative process, of which disorganization is the hallmark.

The crystals were more clearly visible on the IR images in comparison to the FAF images. This has been described earlier. ${ }^{23}$ Gocho et al. identified clusters of hyper-reflective signals and circular spots in the AO images that corresponded to the crystals in three patients with BCD.11 They hypothesized that the circular spots were residual cone photoreceptors located over the crystals. We mostly identified dark spots along the wall of the tubules, which possibly correlated with areas of RPE atrophy.

SD-OCT demonstrates the cross section of the tubules while the same tubules are imaged enface on the $\mathrm{AO}$, appearing as long serpentine tubules. One of the inherent difficulties of imaging on the $r t x 1$ is in patients with cystoid macular changes. ${ }^{10}$ This 
may explain the apparent absence of ORTs in patient 5. Although ORTs were noted in the peripheral macula on the SD-OCT in this patient, this was outside the ROI that we imaged on the AO.

Our study had limitations. Although we attempted to correlate corresponding ORTs on the SD-OCT and AO, we were not able to obtain a good co-registration. Development of a dedicated computer algorithm may help in an accurate co-registration of the ORTs between the SD-OCT and AO, enabling us to understand the morphology better.

This study is an attempt to describe the morphology of the outer retinal tubules on the AO. This ability to directly visualize the living retina provides an implicit advantage in diagnosing, predicting progression and monitoring retinal disease. Longitudinal studies may help observe the progression of these ORTs and correlate better histologically, providing deeper insights into the formation of these structures and therefore pathogenesis of BCD and other retinal degenerative diseases.

\section{References}

1. Bietti GB. Su alcune forme atipiche o rare di degenerazione retinica (degenerazione tappetoretiniche e quadri morbosi similari). Bollettino di Oculistica 1937;16:11591244.

2. Jiao X, Munier FL, Iwata F, et al. Genetic linkage of Bietti crystalline corneoretinal dystrophy to chromosome 4q35. Am J Hum Genet 2000; 67:1309-13.

3. Li A, Jiao X, Munier FL, et al. Bietti crystalline corneoretinal dystrophy is caused by mutations in the novel gene CYP4V2. Am J Hum Genet 2004;74: 817-26.

4. Lee J, Jiao X, Hejtmancik JF, et al. The metabolism of fatty acids in human Bietti crystalline dystrophy. Invest Ophthalmol Vis Sci 2001;42:1707-14.

5. Lai TY, Chu KO, Chan KP, et al. Alterations in serum fatty acid concentrations and desaturase activities in Bietti crystalline dystrophy unaffected by CYP4V2 genotypes. Invest Ophthalmol Vis Sci 2010;51:1092-7.

6. Kaiser-Kupfer MI, Chan CC, Markello TC, et al. Clinical, biochemical and pathologic correlations in Bietti's crystalline dystrophy. Am J Ophthalmol 1994;118:569-582.

7. Zweifel SA, Engelbert M, Laud K, Margolis R, Spaide RF, Freund KB. Outer retinal tubulation: a novel optical coherence tomography finding. Arch Ophthalmol 2009;127:1596-602.

8. Goldberg NR, Greenberg JP, Laud K, Tsang S, Freund KB. Outer retinal tubulation in degenerative retinal disorders. Retina 2013;33:1871-6. 
9. Iriyama AY, Aihara, Yanagi Y. Outer retinal tubulation in inherited retinal degenerative disease. Retina 2013;33:1462-5.

10. Battu R, Dabir S, Khanna A, Kumar AK, Roy AS. Adaptive optics imaging of the retina. Indian J Ophthalmol 2014;62:60-5.

11. Gocho K, Kameya S, Akeo K, et al. High-Resolution Imaging of Patients with Bietti Crystalline Dystrophy with CYP4V2 Mutation. J of Ophthalmol 2014; 2014:1-11

12. Marmor MF, Fulton AB, Holder GE, Miyaki Y, Brigell M, Bach M (2008) Standard for clinical electroretinography (2008 update). Doc Ophthalmol 118:69-77

13. Margolis R, Spaide RF. A pilot study of enhanced depth imaging optical coherence tomography of the choroid in normal eyes. Am J Ophthalmol 2009;147:811-5.

14. Lombardo M, Lombardo G, Ducoli P, Serrao S. Adaptive optics photoreceptor imaging. Ophthalmology 2012.119:1498-1498 e2.

15. Lombardo M, Lombardo G, Ducoli P, Serrao S. Adaptive optics technology for highresolution retinal imaging. Sensors (Basel) 2013;13:334-66.

16. Bennett A, Rudnicka AR, Edgar DF. Improvements on Littmann's method of determining the size of retinal features by fundus photography. Graefes Arch Clin Exp Ophthalmol 1994; 232:361-7.

17. Lombardo M, Serrao S, Ducoli P, Lombardo G. Eccentricity dependent changes of density, spacing and packing arrangement of parafoveal cones. Ophthalmic Physiol Opt 2013;33:516-526.

18. Dabir S, Mangalesh S, Kumar KA, Kummelil MK, Sinha Roy A, Shetty R. et al., Variations in the cone packing density with eccentricity in emmetropes. Eye (Lond) 2014; 28:1488-93.

19. Zhang $Y$, Roorda A. Evaluating the lateral resolution of the adaptive optics scanning laser ophthalmoscope. J Biomed Opt 2006;11:014002.

20. Jung JJ, Freund KB. Long-term Follow-up of Outer Retinal Tubulation Documented by Eye-Tracked and En Face Spectral-Domain Optical Coherence Tomography. Arch Ophthalmol 2012;130:1618-9.

21. Curcio CA, Medeiros NE, Millican CL. Photoreceptor loss in age-related macular degeneration. Invest Ophthalmol Vis Sci 1996;37:1236-49.

22. Schaal KB, Freund KB, Litts KM, Zhang Y, Messinger JD, Curcio CA. Outer Retinal Tubulation In Advanced Age-Related Macular Degeneration: Optical Coherence Tomographic Findings Correspond To Histology. Retina 2015; 0:1-12

23. Halford S, Liew G, Mackay DS, et al. Detailed Phenotypic and Genotypic Characterization of Bietti Crystalline Dystrophy. Ophthalmology 2014;121:1174-84 
TABLE 3.

Details of the cone count on the $\mathrm{A} 0$ imaging in the four quadrants (cone count in cones $/ \mathrm{mm}^{2}$ )

\begin{tabular}{|c|c|c|c|c|c|c|c|c|c|c|c|c|}
\hline \multirow[t]{2}{*}{ Patient } & \multicolumn{2}{|c|}{$1^{0}$ Temporal } & \multicolumn{2}{|c|}{$2^{0}$ Temporal } & \multicolumn{2}{|c|}{$3^{0}$ Temporal } & \multicolumn{2}{|c|}{$1^{0}$ Superior } & \multicolumn{2}{|c|}{$2^{\circ}$ Superior } & \multicolumn{2}{|c|}{$3^{0}$ Superior } \\
\hline & RE & LE & RE & $\mathrm{LE}$ & RE & LE & RE & LE & RE & $\mathrm{LE}$ & RE & LE \\
\hline 1 & 20189 & 22525 & 17320 & 2577 & 17627 & 20613 & 20599 & 20875 & 8554 & 2267 & 19472 & 15769 \\
\hline 2 & 16980 & 8016 & 1944 & 5568 & 7058 & 6580 & 7876 & 4196 & 15786 & 0 & 3971 & 10014 \\
\hline 3 & 0 & 5842 & 491 & 11181 & 295 & 11987 & 295 & 705 & 1065 & 10073 & 3775 & 12692 \\
\hline 4 & 6023 & 1223 & 12849 & 8928 & 16363 & 15744 & 7830 & 5707 & 4774 & 19584 & 7795 & 17568 \\
\hline 5 & 8944 & 808 & 894 & 11628 & 4472 & 14609 & 4969 & 3332 & 3379 & 1111 & 2485 & 5250 \\
\hline $\begin{array}{c}\text { Mean } \\
\pm S D\end{array}$ & $\begin{array}{l}10427.2 \\
\pm 8193\end{array}$ & $\begin{array}{l}7682.8 \\
\pm 8842\end{array}$ & $\begin{array}{r}6699.6 \\
\pm 7833\end{array}$ & $\begin{array}{r}7976.4 \\
\pm 3855\end{array}$ & $\begin{array}{c}9163 \\
\pm 7559\end{array}$ & $\begin{array}{c}13906.6 \\
\pm 5152\end{array}$ & $\begin{array}{l}8313.8 \\
\pm 7530\end{array}$ & $\begin{array}{c}6963 \\
\pm 7986\end{array}$ & $\begin{array}{r}6711.6 \\
\pm 5755\end{array}$ & $\begin{array}{c}6607 \\
\pm 8263\end{array}$ & $\begin{array}{r}7499.6 \\
\pm 6980\end{array}$ & $\begin{array}{l}9710.7 \\
\pm 5814\end{array}$ \\
\hline
\end{tabular}

\section{SUPPLEMENTARY TABLE}

\begin{tabular}{|c|c|c|c|c|c|c|c|c|c|c|c|c|}
\hline Patient & $1^{0} \mathrm{~N}$ & & $2^{0} \mathrm{~N}$ & & & & $1^{\circ} \mathrm{In}$ & erior & $2^{\circ} \mathrm{In}$ & rior & $3^{\circ} \mathrm{In}$ & ferior \\
\hline & RE & LE & RE & LE & RE & LE & RE & LE & RE & LE & RE & LE \\
\hline 1 & 8096 & 1174 & 16910 & 16387 & 17730 & 11337 & 18344 & 20195 & 7261 & 19479 & 15680 & 13089 \\
\hline 2 & 2762 & 2670 & 21103 & 8745 & 17730 & 5896 & 16673 & 3529 & 8769 & 688 & 7805 & 7861 \\
\hline 3 & 607 & 6547 & 16311 & 5264 & 12479 & 8091 & 2632 & 15210 & 6969 & 201 & 11325 & 4029 \\
\hline 4 & 2510 & 3872 & 6131 & 18169 & 2571 & 13251 & 8131 & 3872 & 11326 & 10230 & 11230 & 15979 \\
\hline 5 & 6162 & 2827 & 3469 & 8884 & 6746 & 4341 & 14510 & 202 & 10567 & 230 & 12618 & 15034 \\
\hline $\begin{array}{c}\text { Mean } \\
\pm S D\end{array}$ & $\begin{array}{r}4027.4 \\
\pm 3029\end{array}$ & $\begin{array}{l}5532.8 \\
\pm 3805\end{array}$ & $\begin{array}{l}12784.8 \\
\pm 7577\end{array}$ & $\begin{array}{l}11489.8 \\
\pm 5515\end{array}$ & $\begin{array}{l}11451.2 \\
\pm 6724\end{array}$ & $\begin{array}{l}8583.2 \\
\pm 3702\end{array}$ & $\begin{array}{r}12058 \\
\pm 6540\end{array}$ & $\begin{array}{r}8601.6 \\
\pm 8612\end{array}$ & $\begin{array}{l}8978.4 \\
\pm 1940\end{array}$ & $\begin{array}{r}6165.6 \\
\pm 8581\end{array}$ & $\begin{array}{l}11731.6 \\
\pm 2837\end{array}$ & $\begin{array}{l}11198.4 \\
\pm 5091\end{array}$ \\
\hline
\end{tabular}




\title{
CORRELATION OF STRUCTURE AND FUNCTION OF THE MACULA IN PATIENTS WITH RETINITIS PIGMENTOSA
}

\author{
Rajani Battu, Anjani Khanna, Bharat Hegde, \\ Tos TJM Berendschot, Sandeep Grover, Jan SAG Schouten \\ EYE (LOND) 2015:29(7); 895-901
}





\section{Abstract}

PURPOSE: To correlate the structure of the macula, as measured by spectral-domain optical coherence tomography (SD-OCT) and function, as measured by microperimetry (MAIA) in patients with retinitis pigmentosa (RP) and relatively good visual acuity.

DESIGN: Prospective, cross-sectional, non-intervention study

SUBJECTS: Patients with RP

METHODS: Thirty patients with RP and good central visual acuity were identified. Each patient underwent SD-OCT of the macula and microperimetry. The images were overlaid using the custom-designed software. The retinal sensitivity by microperimetry was correlated with corresponding retinal thickness, as measured by the SD-OCT. ELM, COST and IS/OS junction were scored as intact, disrupted or absent.

MAIN OUTCOME MEASURES: Comparing the retinal sensitivity on the MAIA with various measurements on the SD-OCT.

RESULTS: The retinal sensitivity on the MAIA showed a significant correlation with total retinal thickness and outer retinal thickness on the SD-OCT. There was no association with either the inner retinal thickness or the choroidal thickness. ORT showed a statistical significant correlation with anatomical classification of ELM ( $r=-0.76, P<0.001)$, IS/OS $(r=-0.800, P<0.001), \operatorname{COST}(r=-0.733, P<0,001)$.

CONCLUSION: This study determined that there was a high correlation of the structure and function of the central macula in patients with RP. These studies are important to establish surrogate markers that can be used as end points for various tests in future therapeutic clinical trials. 


\section{Introduction}

Retinitis pigmentosa (RP) is the most common type of inherited retinal degenerative disease characterized by loss of photoreceptors, night blindness, bone spicule-like pigmentary changes in the midperiphery and decreased or undetectable responses on an electroretinogram. ${ }^{1,2} \mathrm{RP}$ is a spectrum of diseases characterized by progressive peripheral visual field constriction that can eventually involve the macula with loss of central visual acuity. There is no effective intervention although several clinical trials are underway to either slow down or arrest the progression of the disease. ${ }^{3-7}$ Progression in RP has traditionally been monitored in the past by assessing visual fields and full-field electroretinography. Although Goldmann visual field testing was the gold standard, more recently static perimetry using the Humphrey Field Analyzer (HFA) (programs 30-2 and 10-2) have been used for this purpose. With the advent of SD-OCT, it has become standard practice to monitor the progression in the structure of the retina, more specifically, the outer retina.

Microperimetry, or fundus-related perimetry is a technique that involves visual field testing while observing the retina simultaneously. This allows observation of the point on the retina that is being stimulated. This is an especially useful method to assess visual fields in patients with unstable or eccentric fixation caused by macular pathologies. ${ }^{8}$ Literature suggests that retinal sensitivity, and not visual acuity, is related to quality of life and has shown to be sensitive to change of function in a trial in RP patients in contrast to HFA $10-2.9^{9-11}$

SD-OCT can show cross-sectional images of the retina with an axial resolution of up to $5 \mu \mathrm{m} .{ }^{12}$ This resolution enhances visualization of the various retinal layers, particularly at the level of the external limiting membrane (ELM) and photoreceptor inner segment/ outer segment (IS/OS) junction. ${ }^{13}$ Several studies have highlighted the importance of the IS/OS layer integrity in studying the progression of RP. ${ }^{13-15}$ Studies have also shown the gradual reduction in the IS/OS layer assessed by the fundus autofluorescence (FAF) ring. ${ }^{16}$

In a study in 2011, Lupo et al. ${ }^{17}$ studied the relation between SD-OCT and microperimetry in RP. Eva et al. investigated the ultrastructural details on the SD-OCT and compared them to the function of the macula, as measured by the microperimetry in these patients. ${ }^{18}$ The purpose of the current study was to perform a structure-function correlation of the retinal sensitivity on the MAIA to thickness of retinal layers and structural changes in the IS/OS, cone-outer segment tips (COST) layer, and ELM on the SD-OCT. 


\section{Materials and Methods}

The study was a prospective case series, approved by the ethics committee of Narayana Nethralaya, Bangalore, India. All the patients consented for the study, and for publication of their data and procedures adhered to the tenets of Declaration of Helsinki. Thirty-four eyes of 34 patients diagnosed with non-syndromic RP based on clinical examination, visual field measurements and electrophysiology were enrolled into the study. The visual acuity in each eye was measured using the Snellen's visual acuity chart. The eye with the better central visual acuity was selected; if both were the same, the right eye was selected. Patients with vision less than 6/36 on the Snellen chart, macular lesions or macular edema on fundus examination or SD-OCT were excluded to ensure a stable fixation during microperimetry and to exclude lower microperimetry values due to macular edema. Thirty eyes were used for statistical analysis.

All patients underwent microperimetry on the MAIA (CenterVue, Padova, Italy) using a predefined pattern that covered the central 100. The Macular Integrity Assessment or MAIA microperimeter uses a scanning laser to perform retinal imaging and an LED light as a stimulus. The eye tracking system on the MAIA was used to ascertain a stable fixation during the entire study. Points corresponding to the foveal centre and the horizontal and vertical lines were used for statistical analysis. Patients who could not maintain a steady fixation underwent a repeat testing, and the second test was used for analysis if fixation was stable. Fixation was considered stable if the reliability index was $>60 \%$. If there was no fixation in the fovea, patients were excluded.

SD-OCT was performed with the Spectralis (Heidelberg Engineering, Heidelberg, Germany). The simultaneous confocal scanning laser ophthalmoscope (CSLO) on the Spectralis allowed precise localization of the anatomical landmarks. The eye tracking system ensured a stable fixation during the entire test. Multiple single scans were averaged at the same location to obtain an increased signal-to-noise ratio. A 100-ART vertical and horizontal scan centred on the fovea was obtained on the SD-OCT for each patient within an area of central $30^{\circ}$. Enhanced-depth imaging scans were obtained to assess the choroidal thickness.

An overlay was prepared using a customized software. The MAIA images were overlaid on the CSLO images of the OCT and matched using blood vessels as a co-registration marker. Structural details on the SD-OCT corresponding to the retinal sensitivity at the central (foveal), $1^{0}, 3^{\circ}$ and $5^{\circ}$ horizontal and vertical lines on the MAIA were compared (Figure 1). 
The inner and outer retinal thickness, and the choroidal thickness on the SD-OCT were assessed at each point corresponding to the central, and six horizontal and vertical points within the central 100 on the MAIA. Two observers (RB, AK) made the measurements independent of each other. The mean values were calculated and used in the analyses. In case there was a difference of 20 um or more between the two observers, the patient was excluded $(n=4)$. On the SD-OCT the ELM, COST layer, IS/OS were assessed and classified as intact, interrupted or absent according to the methods used by others. ${ }^{18}$

The outer retinal thickness (ORT) was measured between the outer border of the retinal pigment epithelium (RPE) and the inner border of the outer plexiform layer $(\mathrm{OPL})$, to include the inner segments, outer segments and the outer nuclear layer; the inner retinal thickness (IRT) was measured between the vitreoretinal interface and the inner border of the OPL. ${ }^{19}$ Submacular choroidal thickness (CT) was measured manually from the inner border of the sclera to the outer border of the RPE vertically using the calipers of the Heidelberg reader software. ${ }^{20}$

\section{FIGURE 1.}

The MAIA of the right eye of a subject overlaid on the CSLO image of the SD-OCT (100 ART horizontal scan) with coregistration of the blood vessels using a customized software. The point measured corresponds to the 50 temporal location on the MAIA (white arrow) and the corresponding measurement on the OCT (yellow arrow).
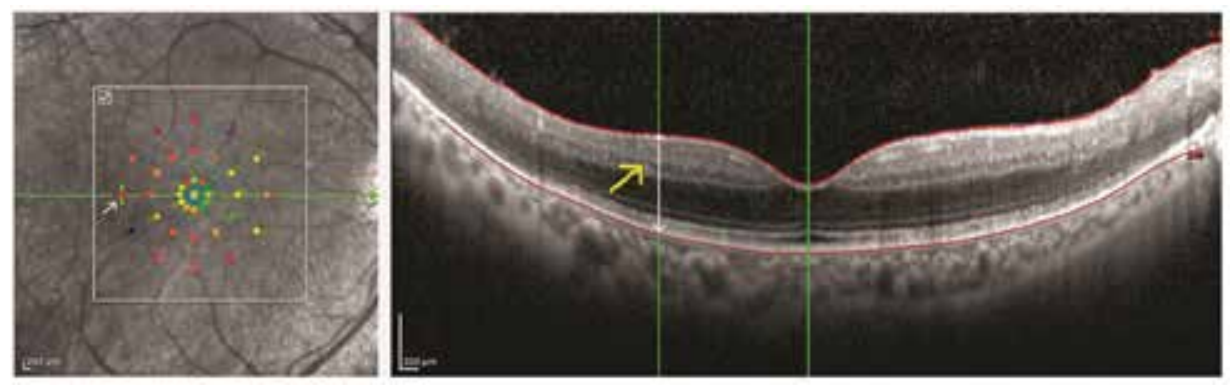

\section{Statistical Analysis}

For statistical analysis Snellen visual acuity was converted to LogMAR units. To determine the relation between retinal sensitivity on the microperimetry and the retinal thickness at corresponding locations on the SD-OCT, the IRT, ORT, the choroidal thickness, ELM, IS/OS and COST abnormalities at different eccentricities were measured. A linear Mixed Models (LMM) analysis was performed with subject ID as grouping factor, retinal sensitivity as independent variable and SD-OCT values, 
eccentricity and site (foveal, inferior, superior, nasal, and temporal) as covariates, as well as their interaction terms. All data were analysed using the statistical software package SPSS version 22.0 (IBM Corp, NY, USA).

\section{Results}

Thirty eyes of 30 patients were studied, 23 males and 7 females. Mean age was $32 \pm$ 12 years (range $12-63$ ) and mean LogMAR visual acuity was $0.19 \pm 0.17$. There was no statistical difference between males and females either in age or LogMAR visual acuity.

On the MAIA, the average reliability index was $94.23 \%$ (range 67-100\%). The average duration to perform the test was 6'52"(range 4'12"-18'51"). The average macular integrity was 90.35 (range 44.3-100). The average threshold was $17.58 \mathrm{~dB}$ (range 2.8-30.1 dB). The mean fixation stabilities were $77.63 \%$ (P1) and $89.80 \%$ (P2). The fixation plot showed that the average bivariate contour ellipse area at $63 \%$ was $2.41^{02}$ and at $95 \%$ was $14.55^{\circ}$ ${ }^{2}$. There was a negative correlation between LogMAR values and sensitivity at the fovea $(r=-0.52, P=0.004)$; the lower the visual acuity, the worse was the retinal sensitivity.

On the SD-OCT, the mean total foveal thickness was $211 \mu \mathrm{m}$ (range 97-281 $\mu \mathrm{m}$ ), the average inner retinal thickness being $122 \mu \mathrm{m}$ (range 62-201 $\mu \mathrm{m}$ ) and outer retinal thickness being $93 \mu \mathrm{m}$ (range $27-119 \mu \mathrm{m}$ ). The mean choroidal thickness in the subfoveal area was $232 \mu \mathrm{m}$ (range $83-439 \mu \mathrm{m})$.

Figure 2 shows the relation between retinal sensitivity and ORT; for all different sites and eccentricities, we found a significant correlation. Figure 3 shows the mean ORT for different sites and eccentricities. LMM analyses revealed a significant association between retinal sensitivity and ORT. This association was different for different sites and eccentricities, that is, showing significant interaction terms.

ELM, COST and IS/OS junction were scored as either intact, disrupted or absent. An analysis of retinal sensitivity by location yielded us a total of 390 locations. We found that ELM was intact in 206, disrupted in 74, and absent in 110 locations.

Similar analysis for IS/OS gave 182, 74 and 134 respectively, and analysis of COST gave 88,68 and 234 respectively. All three layers were intact in 86 , disrupted in 1, and absent in 90 locations. ORT showed a statistically significant correlation with the anatomical classification of ELM $(r=-0.76, P<0.001)$, IS/OS $(r=-0.800, P<0.001)$, and COST $(r=$ $-0.733, \mathrm{P}<0.001)$. 
Sensitivity values at different locations and eccentricities were grouped according to the above anatomical integrity classification, and we found highest mean sensitivities in the intact group, lower for the disrupted group, and lowest for the absent group for all three layers (Table 1).

Bivariate correlation showed that MAIA correlated significantly with sex $(\mathrm{P}<0.001)$, spherical equivalent $(P<0.001)$, Total retinal thickness (TRT) $(P<0.001)$, ORT $(P<0.001)$, $\operatorname{ELM}(P<0.001)$, IS/OS $(P<0.027), \operatorname{COST}(P<0.001)$, location $(P=0.032)$, and eccentricity $(P<0.001)$.

Using the above data, we performed a multivariate regression (Table 2), keeping the retinal sensitivity as the dependent variable, and age, sex, spherical equivalent, ORT, IRT, TRT and anatomy of the ELM, IS/OS and COST, and location and eccentricity of measurement as independent variables. We found that sex, ORT, and the anatomical integrity of the ELM, and IS/OS had an important role in the retinal sensitivity.

\section{FIGURE 2.}

Scatter plot showing the relation between MAIA values of ORT for the different sites (f-fovea, i-inferior, n-nasal, $s$-superior, $t$ - temporal) shown in different rows and eccentricities (columns). The correlation coefficients included are all significant $(\mathrm{P}<0.005)$.

degree

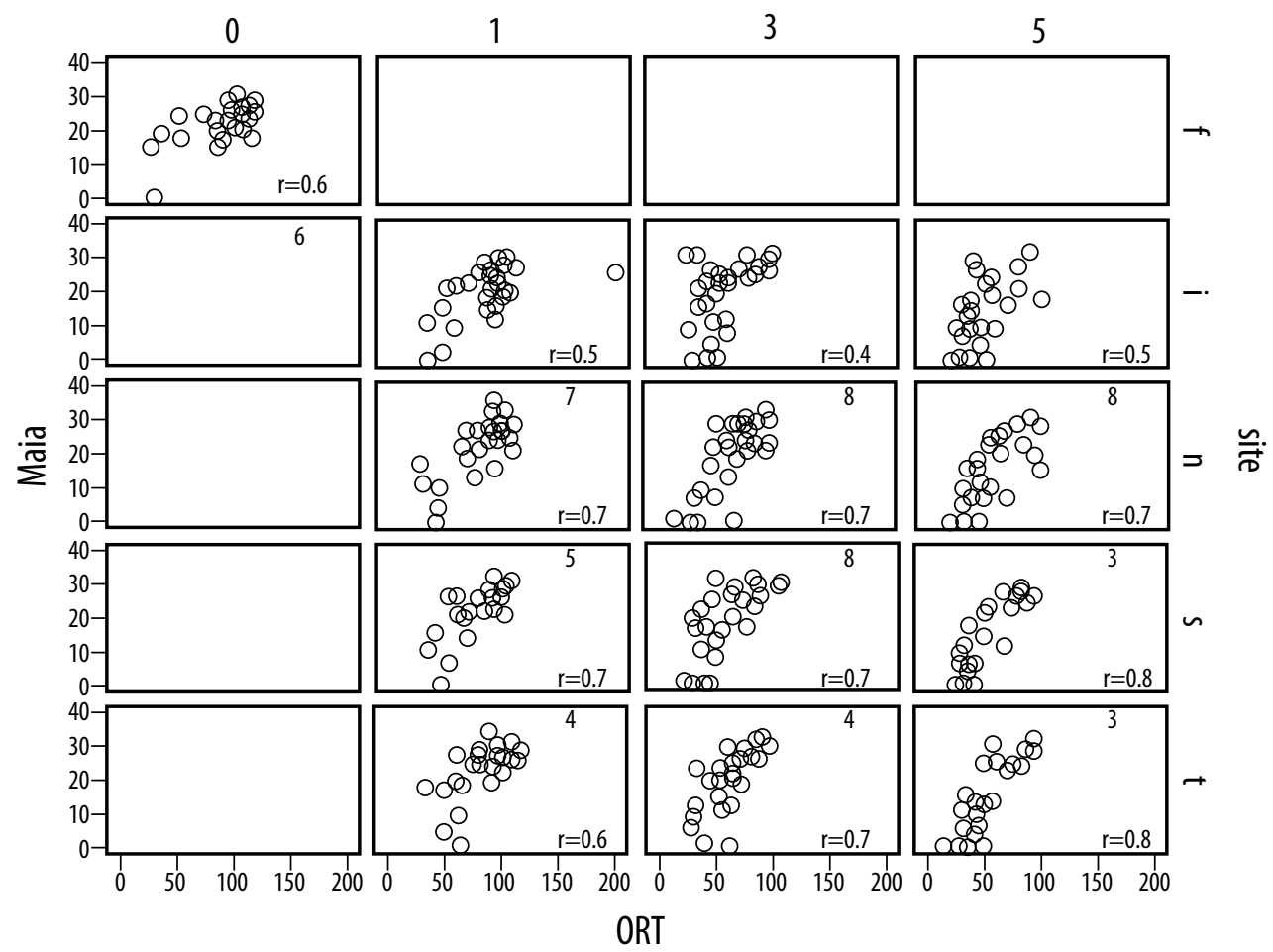


FIGURE 3.

Mean ORT for the different sites. Different lines represent different eccentricities. Similar graphs were drawn for the IRT and EDI (f-fovea, i-inferior, n-nasal, s-superior, t- temporal)

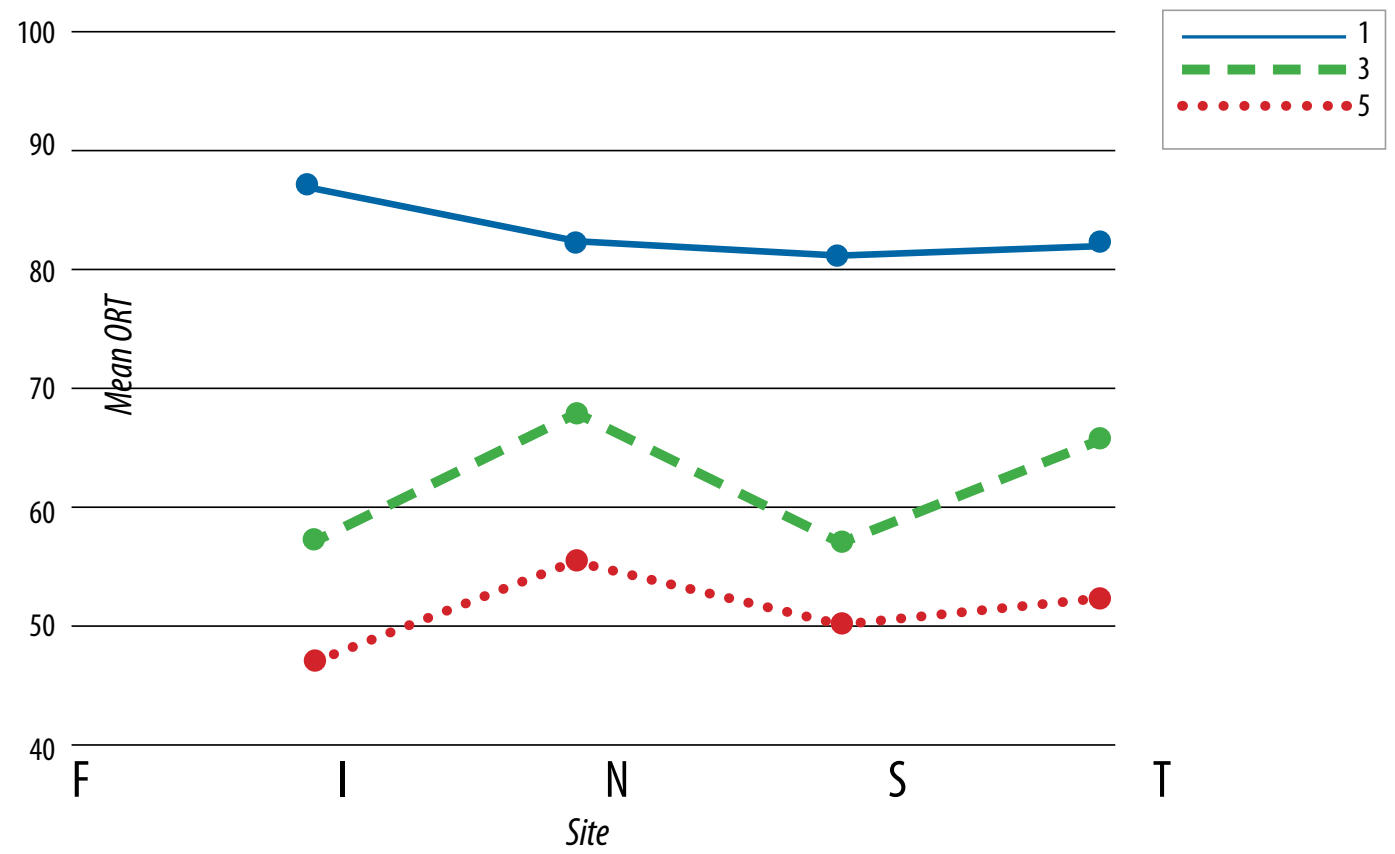


TABLE 1.

Summary of the mean retinal sensitivities using MAIA at different locations and eccentricities grouped by the anatomical status of the outer retinal layers on SD-OCT

\begin{tabular}{|c|c|c|c|c|c|c|c|c|c|c|c|c|c|}
\hline \multirow[t]{2}{*}{ Location } & \multirow{2}{*}{$\begin{array}{c}\text { Eccentricity }^{\mathrm{a}} \\
\mathrm{RE}\end{array}$} & \multicolumn{4}{|c|}{ IS/OS } & \multicolumn{3}{|c|}{ COST } & & \multicolumn{2}{|c|}{ ELM } & \multirow[b]{2}{*}{$A b$} & \multirow[b]{2}{*}{$\mathrm{p}$ value $\mathrm{e}^{\mathrm{b}}$} \\
\hline & & In & Dis & $A b$ & $p$ value $^{b}$ & In & Dis & $A b$ & $p$ value $^{b}$ & In & Dis & & \\
\hline Foveal & 0 & 24.43 & 17.63 & 23.00 & $<0.001$ & 25.93 & 17.86 & 21.00 & 0.004 & 24.39 & 20.20 & 7.50 & $<0.001$ \\
\hline \multirow[t]{3}{*}{ Inferior } & 1 & 24.48 & 17.17 & 3.63 & $<0.001$ & 26.23 & 20.56 & 12.75 & $<0.001$ & 24.14 & 14.33 & 5.50 & $<0.001$ \\
\hline & 3 & 25.78 & 14.00 & 18.00 & 0.58 & 27.67 & 25.00 & 18.00 & 0.176 & 25.00 & 18.11 & 16.09 & 0.100 \\
\hline & 5 & 24.50 & 21.00 & 7.96 & $<0.001$ & 26.67 & 18.00 & 9.46 & 0.016 & 21.29 & 11.00 & 7.95 & 0.010 \\
\hline \multirow[t]{3}{*}{ Superior } & 1 & 25.78 & 12.60 & 12.50 & $<0.001$ & 27.55 & 23.22 & 16.90 & 0.002 & 25.73 & 15.67 & 10.50 & $<0.001$ \\
\hline & 3 & 28.00 & 18.43 & 10.07 & $<0.001$ & 29.67 & 28.50 & 13.87 & 0.004 & 25.75 & 17.13 & 7.60 & $<0.001$ \\
\hline & 5 & 26.80 & 14.00 & 7.53 & $<0.001$ & 27.00 & 26.00 & 9.77 & 0.007 & 23.67 & 10.00 & 6.35 & $<0.001$ \\
\hline \multirow[t]{3}{*}{ Nasal } & 1 & 26.48 & 12.00 & 8.50 & $<0.001$ & 27.60 & 23.30 & 17.70 & 0.025 & 25.52 & 16.33 & 12.50 & 0.003 \\
\hline & 3 & 26.50 & 6.20 & 14.43 & $<0.001$ & 28.50 & 25.80 & 16.26 & 0.018 & 26.17 & 10.86 & 12.40 & $<0.001$ \\
\hline & 5 & 23.88 & 12.00 & 10.42 & 0.003 & 27.67 & 21.00 & 11.63 & 0.011 & 20.69 & 11.00 & 8.62 & 0.004 \\
\hline \multirow[t]{3}{*}{ Temporal } & 1 & 25.71 & 17.13 & 19.00 & 0.021 & 27.64 & 24.57 & 18.33 & 0.010 & 25.00 & 22.20 & 11.67 & 0.015 \\
\hline & 3 & 26.46 & 20.14 & 14.00 & 0.002 & 30.60 & 27.00 & 16.32 & $<0.010$ & 26.12 & 19.29 & 7.67 & $<0.001$ \\
\hline & 5 & 26.29 & 15.33 & 7.65 & $<0.001$ & 29.67 & 20.33 & 10.67 & 0.004 & 25.38 & 15.33 & 6.94 & $<0.001$ \\
\hline
\end{tabular}

ABBREVIATIONS

COST, cost outer segment tips; ELM, external limiting membrane; IS/OS, inner segment/outer segment junction.

a eccentricity measured in degrees from fovea. b comparison of means-One-way Anovaln-intact, Dis-disrupted,

Ab-absent 
TABLE 2.

Multivariate linear regression model for dependent variable : retinal sensitivity in $\mathrm{db}$ measured with the MAIA (Model $\left.R^{2}=0.607\right)$

\begin{tabular}{|l|c|c|c|}
\hline \multicolumn{1}{|c|}{ Parameter } & beta & Standardised beta & p value \\
\hline Intercept & 25.15 & & $<0.001$ \\
\hline Age (years) & -0.05 & -0.059 & 0.112 \\
\hline Sex (male, female) & -4.64 & -0.192 & $<0.001$ \\
\hline Sph Eq (in diopters) & -0.162 & -0.042 & 0.251 \\
\hline TRT (in $\mu \mathrm{m}$ ) & -0.050 & -0.266 & 0.542 \\
\hline ORT (in $\mu \mathrm{m})$ & 0.175 & 0.467 & 0.043 \\
\hline IRT (in $\mu \mathrm{m})$ & 0.070 & 0.372 & 0.393 \\
\hline ELMa & -2.909 & -0.248 & $<0.001$ \\
\hline ISOS & -1.942 & -0.170 & 0.006 \\
\hline COSTa & -0.490 & -0.040 & 0.442 \\
\hline EDla & -0.004 & -0.033 & 0.385 \\
\hline Locationb & -0.052 & -0.006 & 0.856 \\
\hline Eccentricityc & -0.663 & -0.062 & 0.252 \\
\hline
\end{tabular}

ABBREVIATIONS

COST, cone outer segment tips; EDI, enhanced depth imaging of the choroid; ELM, external limiting membrane; ; IRT: Inner retinal thickness;; ISOS: Inner segment outer segment junction; ORT: Outer retinal thickness; Sph Eq: Spherical equivalent; TRT: Total retinal thickness;:;

a intact-1, disrupted-2, absent-3

${ }^{b}$ fovea-1, temporal-2, nasal-3, superior-4, inferior-5

$\left(7^{0}-1,3^{0}-2,5^{0}-3\right.$

\section{Discussion}

Lupo et al. ${ }^{17}$ studied the relationship between retinal thickness and retinal function in RP.They, however, only compared the central retinal thickness with the retinal sensitivity on the microperimetry and did not look at the structural details. A study by Eva et al. assessed the correlation of visual function measured by both static and kinetic fundusrelated perimetry with structural changes in the inner and outer retina evaluated with SD-OCT and with molecular changes in the RPE visualized with FAF. ${ }^{18}$ Mitamura et al. ${ }^{21}$ found a significant correlation between the length of the IS/OS line and the retinal sensitivity using the MP-1 microperimeter. 
Studies to establish the structure-function correlation in retinal degenerative diseases are essential as more and more treatment modalities become available. We developed a custom-made software to assess the structure-function correlation between fundusrelated microperimetry and SD-OCT. We found that the retinal sensitivity correlated with the outer retinal structure and an intact ELM and IS/OS junction but not with the inner retinal thickness, choroidal thickness or the COST layer.

Microperimeters allow for registration of fundus photos with the other imaging modalities and make it possible to compare retinal morphology with visual function. ${ }^{22}$ Retinal sensitivity assessed using microperimetry has been advocated as a potential outcome measure of macular function for clinical trials involving patients with RP. ${ }^{23}$ Cideciyan et al. ${ }^{23}$ have established repeatability of microperimetry in patients with RP. In their cohort of patients with ABCA4-associated retinopathy, that included RP, they showed that repeated microperimeter tests showed reasonable concordance and the test-retest variance was not significant. Changes on the SD-OCT have been used in several studies to study the disease progression, ${ }^{13-16}$ and by the same measure may be useful in monitoring response to therapy.

$\mathrm{RP}$ is heterogeneous group of diseases, mutations in more than 50 genes are known to cause non-syndromic RP and more than 3100 mutations have been identified in these genes. $^{24,25}$ The gene products involve multiple metabolic pathways localized to mostly rods, or rods and cones, or the retinal pigment epithelium. ${ }^{2}$ These include proteins of rod visual transduction, photoreceptor differentiation, proteins of the cytoskeleton, protein transportation and retinol metabolism among others. ${ }^{2}$ Mutations in any of the biochemical cycles that alter the phototransduction cascade, including renewal and shedding of the photoreceptor outer segments, visual transduction, and/or retinol metabolism may cause retinitis pigmentosa. ${ }^{26}$

Our study showed that the retinal sensitivity in the macula correlated well with the outer retinal thickness mainly, which included the inner and outer segments of the photoreceptors. It is not surprising that many important biochemical processes occur in the outer retinal layers, and structural preservation of these layers corresponds to a functional preservation.

Issa et al. ${ }^{27}$ showed that in one patient with Usher's syndrome, there was a significant relationship between outer retinal thickness and retinal sensitivity. They demonstrated that good functional preservation was associated with outer retinal thickness more than $50 \mu \mathrm{m}$ in spite of extensive disruption of the inner retina. 
The study by Lenassi et al. ${ }^{18}$ found a high correlation between retinal sensitivity and outer retinal thickness, than overall retinal thickness in patients with RP. We found that the retinal sensitivity correlates well with both outer and total retinal thickness and not inner retinal thickness or the choroidal thickness. Changes in the choroid are probably secondary to changes that occur in the retinal layers, and therefore choroidal thickness may vary in patients with RP.

In addition to the IS/OS line, the integrity of the ELM and COST layers have been described as potential indicators of retinal function; a relation between intact ELM/ COST layer and good visual acuity has been described in degenerative eye diseases like age-related macular degeneration, macular hole and epiretinal membrane. ${ }^{28-31}$

We found that the presence of intact ELM and an intact IS/OS correlated significantly with the retinal sensitivity. Oishi et al. ${ }^{29}$ found a strong correlation between ELM status and visual acuity that was higher than that with the IS/OS in a cohort of patients with age-related macular degeneration (AMD). The status of the ELM was the most important factor for visual acuity. In RP, it is possible that in addition to the IS/OS, the ELM is a marker of photoreceptor integrity. Progressive degeneration of photoreceptors may lead to loss of inner retinal structures, and loss of ELM may indicate a severe degree of damage.

In a similar study, Itoh et al..$^{28,30}$ found a significant correlation between the presence of an intact COST line and visual recovery in patients who underwent surgery for macular hole. In an earlier study, Srinivasan et al. ${ }^{32}$ had shown that the cone outer segment lengths were greatest in the foveola and decreased with increasing eccentricity. We did not find a significant association between the COST layer and the retinal sensitivity.

Functional mapping of the macula is essential in retinal degenerative diseases. It allows us a window of opportunity to treat before permanent structural damage occurs. It also helps select patients with early functional loss in whom there are better chances of success with an experimental therapy and help efficient monitoring of the effectiveness of any therapy in RP. Although visual acuity is considered to be the standard primary end point in clinical trials of retinal diseases, it is important that other end points be considered in diseases like RP. This study is one such attempt to identify surrogate end points, which may be useful to monitor future therapies. Sugawara et al $^{9}$ have established that macular sensitivity determined by microperimetry may be a good predictor of quality of life in RP patients with relatively good vision. Quality of life needs to be an important consideration when future clinical studies and pharmaceutical regulations are considered. 
Studies like this help us understand better the structural changes that occur in RP. It would be interesting to study the natural course of the disease and assess the rate of functional loss vis-à-vis the morphological changes in the structure. This may be an important consideration for pharmaceutical interventions. Our study is an attempt to establish the markers that help identify early structural damage before functional loss is noted. This may further help us understand how soon we need to intervene in the course of the disease for it to prevent irretrievable visual loss. Identifying these markers is important in formulating the inclusion and exclusion criteria for clinical trials in RP.

It is hard to predict whether functional changes precede structural changes in RP. A co-registration of the MAIA and SD-OCT shows the early functional changes associated with micro-structural alterations. Issa et al. ${ }^{27}$ suggested that microstructural alterations precede functional loss, and high-resolution retinal imaging can detect this. If structural changes do precede functional loss, and it is possible to detect these changes on a high-definition imaging device, this would be of vital importance in patients with RP, as it would mean that therapy can be offered to these patients before they notice a functional visual loss. Adaptive optics may be one such imaging device that may pick up ultrafine structural changes. ${ }^{33} \mathrm{~A}$ structure function correlation between adaptive optics and microperimetry may be useful to detect the earliest structural changes in RP and other such degenerative retinal disorders.

Our study has limitations. The sample size was small, and it was a cross-sectional study. We did not have the results of genetic studies of these patients.

There is a continuous need to detect degenerative diseases like RP in its earliest stage, when therapy may be most useful and before irreversible damage has occurred. Studies like ours try to identify surrogate end points to monitor response to clinical trials before true functional loss has occurred.

\section{Summary}

\section{WHAT WAS KNOWN BEFORE:}

- Structure-function correlation in RP. The central retinal thickness on the SD-OCT correlates with the retinal sensitivity on the microperimeter.

- Correlation with outer retinal thickness. Outer retinal thickness correlates with the retinal sensitivity on the OCT. 


\section{WHAT THIS STUDY ADDS:}

- In RP, an intact ELM and an intact IS/OS correlated significantly with the visual acuity and retinal sensitivity.

- An intact COST layer does not correlate with the retinal sensitivity.

\section{References}

1. Fishman GA. Retinitis pigmentosa. Genetic percentages. Arch Ophthalmol 1978; 96:822-826.

2. Hamel C. Retinitis pigmentosa. Orphanet J Rare Dis 2006; 1:40.

3. Humayun MS, Dorn JD, da Cruz L, et al. Argus II Study Group. Interim results from the international trial of Second Sight's visual prosthesis. Ophthalmology 2012; 119:779788.

4. Tan MH, Smith AJ, Pawlyk B, et al. Gene therapy for retinitis pigmentosa and Leber congenital amaurosis caused by defects in AIPL1: effective rescue of mouse models of partial and complete Aip/ 1 deficiency using AAV2/2 and AAV2/8 vectors. Hum Mol Genet 2009; 18: 2099-2114.

5. Bennett J. Gene therapy for retinitis pigmentosa. Curr Opin Mol Ther 2000; 2: 420-425.

6. Chader GJ, Weiland J and Humayun MS. Artificial vision: needs, functioning, and testing of a retinal electronic prosthesis. Prog Brain Res 2009; 175:317-332.

7. MacLaren RE, Groppe M, Barnard AR, et al. Retinal gene therapy in patients with choroideremia: initial findings from a phase 1/2 clinical trial. Lancet 2014; 383: 11291137.

8. Rohrschneider KS, Bultmann and Springer C. Use of fundus perimetry (microperimetry) to quantify macular sensitivity. Prog Retin Eye Res 2008; 27: 536-548.

9. Sugawara T, Sato E, Baba T, Hagiwara A, Tawada A, Yamamoto S. Relationship between vision-related quality of life and microperimetry-determined macular sensitivity in patients with retinitis pigmentosa. Jpn J Ophthalmol 2011; 55: 643-646.

10. Sugawara T, Hagiwara A, Hiramatsu A, Ogata K, Mitamura Y, Yamamoto S. Relationship between peripheral visual field loss and vision-related quality of life in patients with retinitis pigmentosa. Eye 2010; 24: 535-539.

11. Tawada A, Sugawara T, Ogata K, Hagiwara A, Yamamoto S. Improvement of central retinal sensitivity six months after topical isopropyl unoprostone in patients with retinitis pigmentosa. Indian J Ophthalmol 2013; 61: 95-99.

12. Alam S, Zawadzki RJ, Choi S, et al. Clinical application of rapid serial fourier- 
domain optical coherence tomography for macular imaging. Ophthalmology 2006;113:1425-1431.

13. Aizawa S, Mitamura Y, Baba T, Hagiwara A, Ogata K, Yamamoto S. Correlation between visual function and photoreceptor inner/outer segment junction in patients with retinitis pigmentosa. Eye 2009; 23:304-308.

14. Fischer M, Fleischhauer JC, Gillies MC, Sutter FK, Helbig H, Barthelmes D. A new method to monitor visual field defects caused by photoreceptor degeneration by quantitative optical coherence tomography. Invest Ophthalmol Vis Sci. 2008; 49:3617-3621.

15. Jacobson SG, Roman AJ, Aleman TS, et al. Normal central retinal function and structure preserved in retinitis pigmentosa. Invest Ophthalmol Vis Sci 2010; 51:1079_ 1085.

16. Robson AG, Michaelides M, Saihan Z, et al. Functional characteristics of patients with retinal dystrophy that manifest abnormal parafoveal annuli of high density fundus autofluorescence; a review and update. Doc Ophthalmol 2008; 116: 79-89.

17. Lupo S, Grenga PL, Vingolo EM. Fourier-domain optical coherence tomography and microperimetry findings in retinitis pigmentosa. Am J Ophthalmol 2011; 151:106111.

18. Lenassi E, Troeger E, Wilke R and Hawlina M. Correlation between Macular Morphology and Sensitivity in Patients with Retinitis Pigmentosa and Hyperautofluorescent Ring. Invest Ophthalmol Vis Sci 2012; 53:47-52.

19. Curcio C, Messinger JD, Sloan KR, Mitra A, McGwin G, Spaide R. Human chorioretinal layer thicknesses measured in macula-wide, high-resolution histologic sections. Invest Ophthalmol Vis Sci 2011; 52:3943-3954.

20. Dhoot DS, Huo S, Yuan A, et al. Evaluation of choroidal thickness in retinitis pigmentosa using enhanced depth imaging optical coherence tomography. $\mathrm{Br} J$ Ophthalmol 2013; 97: 66-69.

21. Mitamura Y, Aizawa S, Baba T, Hagiwara A, Yamamoto S. Correlation between visual function and photoreceptor inner/outer segment junction in patients with retinitis pigmentosa. Br J Ophthalmol 2009; 93: 126-127

22. Acton JH, Vivienne C and Greenstein VC. Fundus-driven perimetry (microperimetry) compared to conventional static automated perimetry: similarities, differences, and clinical applications. Can J Ophthalmol 2013; 5: 358-363.

23. Cideciyan A, Swider M, Aleman TS, et al. Macular function in macular degenerations: repeatability of microperimetry as a potential outcome measure for ABCA4associated retinopathy trials. Invest Ophthalmol Vis Sci 2012; 53: 841-852. 
24. HGMD, Human Gene Mutation Database (Biobase Biological Databases) 2013. Retrieved from http://www.hgmd.cf.ac.uk/. Accessed June 2014.

25. Retnet, The Retinal Information Network 2013. Retrieved from http://www.sph.uth. tmc.edu/RetNet/. Accessed June 2014. 2014.

26. Wert KJ, Lin JH and Tsang SH. General pathophysiology in retinal degeneration. Dev Ophthalmol 2014; 53: 33-43.

27. Issa PC, Troeger E, Finger R, Holz FG, Wilke R, Scholl HP. Structure-function correlation of the human central retina. PLoS One 2010; 5:12864.

28. Itoh Y, Inoue M, Rii T, Hiraoka T, Hirakata A. Significant Correlation Between Visual Acuity and Recovery of Foveal Cone Microstructures After Macular Hole Surgery. Am J Ophthalmol 2012; 153:111-119.

29. Oishi A, Hata M, Shimozono M, Mandai M, Nishida A, Kurimoto Y.The Significance of External Limiting Membrane Status for Visual Acuity in Age-Related Macular Degeneration. Am J Ophthalmol 2010; 150: 27-32.

30. Itoh Y, Inoue M, Rii T, Hiraoka T, Hirakata A, Correlation between Length of Foveal Cone Outer Segment Tips Line Defect and Visual Acuity after Macular Hole Closure. Ophthalmology 2012; 119:1438-1446.

31. Shimozono M, Oishi A, Hata M, et al. The Significance of Cone Outer Segment Tips as a Prognostic Factor in Epiretinal Membrane Surgery. Am J Ophthalmol 2012; 153: 698-704.

32. Srinivasan VJ, Monsoon BK, Wojtkowski M, et al. Characterization of outer retinal morphology with high-speed, ultrahigh-resolution optical coherence tomography. Invest Ophthalmol Vis Sci 2008; 49: 1571-1579.

33. Battu R, Dabir S, Khanna A, Kumar AK, Roy AS. Adaptive optics imaging of the retina. Indian J Ophthalmol 2014; 62: 60-65. 



\section{RETINAL OXYGEN SATURATION IN RETINITIS PIGMENTOSA AND MACULAR DYSTROPHIES IN ASIAN INDIAN EYES}

Rajani Battu, Ashwin Mohan, Anjani Khanna, Abhinav Kumar, Rohit Shetty INVESTIGATIVE OPHTHALMOLOGY AND VISUAL SCIENCE 2015:56(5); 2798-2802 



\section{Abstract}

PURPOSE: To study the oxygen saturation profiles in RP and macular dystrophies and compare them with age-matched health controls.

METHODS: In a cross sectional prospective study, 62 subjects with RP, 23 with macular dystrophies and 78 controls were enrolled and retinal oximetry was performed with the Oxymap T1 retinal oximeter. The images were analyzed for oxygen saturation and diameter of retinal vessels

RESULTS: All parameters showed a significant difference between the three groups. Patients with RP showed significantly lower diameters $(98.4 \mu \mathrm{m}$ and $136.9 \mu \mathrm{m}$ arteriolar and venous) $(P<0.001)$, higher saturations $(102.3 \%$ and $59.1 \%)(P<0.001 ; 00.06)$ and higher arterio-venous saturation difference (AVSD) $(43 \%)(P<0.001)$ compared with the other two groups. Macular dystrophies showed higher global arteriolar values (96.7\%) and AVSD (41.6\%) but comparable venous values (54.9\%) to the control group $(90.6 \%$, $57.4 \%$ and $33.3 \%)$.

CONCLUSIONS: Oximetry is sensitive in quantifying hemodynamic changes in retinal dystrophies. It is still unclear whether these hemodynamic changes are a cause or a result of the disease process. 


\section{Introduction}

Retinitis pigmentosa (RP) encompasses a large group of hereditary diseases of the posterior segment of the eye characterized by degeneration, atrophy and finally loss of photoreceptors and retinal pigment epithelium (RPE), leading to progressive visual loss. ${ }^{1}$ The prevalence of RP is estimated to be 1 in 4000 individuals, with a total of about two million affected persons worldwide. ${ }^{2,3}$ The prevalence has been reported to be higher in the Indian population. ${ }^{4}$

Hereditary macular disorders are characterized by defects of the cone photoreceptors or RPE underlying the macula, and include Stargardt disease, cone dystrophy, cone-rod dystrophy and other maculopathies. ${ }^{5}$ Many genes affecting the photoreceptors have been implicated in the pathogenesis of these diseases. ${ }^{1,5} \mathrm{Although}$ the exact mechanism of cell death in RP is not known, oxidative stress is known to play a major role. ${ }^{6}$

Disturbed ocular blood flow has been described as another potential causative factor in RP.' This is an interesting hypothesis as reduced ocular blood flow can either occur as a primary event and cause ischemia and tissue loss, or occur as a physiological secondary response to reduced tissue and demand. Konieczka et al. have suggested that reduction in ocular blood flow in RP patients could be a primary event. ${ }^{1}$

An early ocular hemodynamic finding in patients with RP is increased arterio-venous (A-V) transit time and reduced blood flow velocity which have been observed before any clinically detectable ocular pathology. ${ }^{7}$ Doppler imaging has demonstrated that peak systolic flow velocities are decreased in both ophthalmic arteries and posterior ciliary arteries. ${ }^{8}$ Interestingly this decrease in blood flow velocity was not confined to the ocular circulation. Systemic findings like reduced flow in the cutaneous capillaries of the finger and longer recovery time after cold provocation has been observed in RP patients. ${ }^{8}$

There is evidence that in RP patients, ocular blood flow is reduced beyond what is attributable to reduction secondary to retinal atrophy. Konieczka et al.' hypothesize that the primary ocular blood flow is reduced in RP patients due to Peripheral vascular dysregulation syndrome (PVD). Celline et al. ${ }^{8}$ have further demonstrated a disturbance in peripheral blood flow in addition to that in the eye in RP patients. Reduced blood flow in the retina and an increase in the AV transit time have been observed before the appearance of any ophthalmoscopic signs.? In summary, the observation of 
an association with PVD syndrome and peripheral vascular abnormalities and the increased AV transit time occurring early in the disease argue strongly in favour of vascular disturbances being a primary event.

Apart from blood flow velocity and vascular diameters, it is possible to measure the saturation of oxygen in the hemoglobin contained in the ocular vessels. A photospectrometric device measures oxygen saturation in a non-invasive manner.9 An increase in venous saturations and a resultant decrease in AVSD in eyes with RP have been reported. ${ }^{10,11}$

A large majority of genes associated with cone dystrophies are yet to be discovered; this hints towards the existence of unknown cone-specific or cone-sensitive processes. ${ }^{5}$ No established evidence exists to implicate ocular blood flow abnormalities in the pathogenesis of cone dystrophies. It would therefore be interesting to study the ocular blood flow and oxygen saturations in cone dystrophies.

We aimed to study and compare the oxygen saturation profiles and vascular diameters in RP and macular dystrophies and compare them with age- matched controls.

\section{Materials and Methods}

A total of 114 consecutive patients presenting to the retina department at Narayana Nethralaya, Bangalore, diagnosed with RP and cone dystrophy or Stargardt disease were enrolled in the study. The diagnosis of these conditions was made based on the presentation, clinical features and electrophysiological examination. A detailed history was taken for every patient; those with cardiovascular disease, diabetes, hypertension, or history of migraine or using any ocular or systemic medications were excluded. The study followed the tenets of Helsinki. The ethics committee and institutional review board of Narayana Nethralaya approved the study. All patients gave a written consent for the study.

All patients underwent a complete ophthalmic examination. These included measurement of the best-corrected visual acuity, anterior segment examination, measurement of the intraocular pressures and a fundus examination. All of them underwent fundus photography, spectral domain optical coherence tomography (SDOCT) and full- field ERG. Patients younger than 18 years of age, smokers, with a previous history of trauma, nystagmus and poor media clarity were excluded from the study. 
This article summarizes the oximetry findings; the SD-OCT and ERG findings were used only for confirmation of the diagnosis. They have not been included, as it would confound the reader with too much information.

\section{Image Acquisition}

Following dilation with $1 \%$ tropicamide and $10 \%$ phenylephrine, all patients underwent dual wavelength photospectrometric Oximetry (Oxymap T1 retinal oximeter, Oxymap, Reykjavik, Iceland).

The initial images were obtained after the patients were allowed to rest for 5 minutes to eliminate exercise-induced fluctuations in readings. Resting blood pressure and pulse oximetry ( $\mathrm{HE}$; Silicon Labs, Chennai, India) readings were measured in all patients. None of the subjects had consumed caffeine within 2 hours of the examination. The aiming light was set at the lowest setting, flash intensity was 50W, small aperture and large pupil settings were applied to the Topcon TRC 50DX Fundus camera (Topcon, Tokyo, Japan)

One experienced photographer obtained standardized images for all the subjects. We obtained 2 images per eye of $50^{\circ}$ field that were disc centred in all subjects. (Figure 1) We ensured that all the images analyzed were in sharp focus. To achieve this, we chose the best quality image between the 2 eyes in bilateral cases, which not only ensured quality, but also ensured that we take only one reading per patient eliminating duplication of data. In unilateral cases or cases with only one available image, the best quality image was taken. If no image appeared satisfactory, or the eye had measurable vessels in only one quadrant, that patient was excluded from the study ( $n=29$ ). A circle was drawn concentric to the optic disc, leaving 50 pixels form the disc margin. A second concentric circle was drawn twice the diameter of the first one. Vessel segments were analyzed between these two concentric circles to ensure that retinal eccentricity was uniform. Retinal eccentricity and cell density may have an impact on measured retinal saturations. Images were analyzed by choosing the thickest arteriole and venule representative of that quadrant.

\section{Statistical Analysis}

Statistical analysis was done using IBM SPSS v22.0 (IBM SPSS Statistics; IBM Corporation, Chicago, IL, USA). All parameters were tested for normality using the Shapiro-Wilk Test. 
Parametric data was analyzed using the One-way ANOVA with the Turkey test for posthoc analysis. Non-parametric data was compared using the Kruskal-Wallis test and the Mann-Whitney $U$ test for the post-hoc analysis.

\section{Results}

There were a total of 23 eyes with macular dystrophies, 62 eyes with retinitis pigmentosa and 78 controls.

\section{Non-measurable Vessels}

Analyzable vessels were present in all quadrants in the macular and control group. The RP group had 14 eyes without measurable arterioles in both the superonasal and inferonasal quadrants, 15 eyes without infero-nasal arterioles only, 5 eyes without supero-nasal arterioles only, 1 eye without supero-nasal venule and 3 eyes without infero-nasal venules. In all quadrants where the venules were not measurable, the corresponding arterioles were definitely not measurable, although the reverse was not true. In 32 eyes, the arterioles were not measurable but the venules were measurable. In all, 34 eyes/62 (54.8\%) had non-measurable vessels in the nasal hemifield.

In our study, we found that the nasal vessels attenuated earlier compared with the temporal ones. In those eyes without measurable temporal vessels, the nasal ones were also not measurable, This accounted for the 7 out of the 29 eyes that we had to exclude because both the temporal and nasal vessels were not measurable.

\section{Demographics}

The average age in the macular dystrophy group was $20.8 \pm 11.5$, RP group was 30.4 \pm 16.7 and the control group was $32.4 \pm 9.4$. Age matched non-smoking controls were chosen according to the RP, as the numbers were larger in that group. The age distribution in the macular group was significantly different from the other two groups. No significant differences were found in the sex distribution. None of the patients had any significant refractive error beyond \pm 0.5 diopter. The IOP for both the RP group (mean 14.5, range 10-18) and the macular dystrophy group (mean 14.4, range 9-18) was within normal ranges. The results have been summarized in Table 1. 
TABLE 1.

Demographics

\begin{tabular}{|l|c|c|c|c|}
\hline \multicolumn{1}{|c|}{$\begin{array}{c}\text { Parameter, } \\
\mathbf{n = 1 6 3}\end{array}$} & $\begin{array}{c}\text { Macular, } \\
\mathbf{n - 2 3}\end{array}$ & $\begin{array}{c}\mathbf{R P}, \\
\mathbf{n = 6 2}\end{array}$ & $\begin{array}{c}\text { Controls, } \\
\mathbf{n = 7 8}\end{array}$ & Significance \\
\hline Age & $\begin{array}{c}20.8 \pm 11.5 \\
(15.8-25.8)\end{array}$ & $\begin{array}{c}30.4 \pm 16.6 \\
(26.2-34.7)\end{array}$ & $\begin{array}{c}32.4 \pm 9.4 \\
(30.3-34.5)\end{array}$ & $<0.001^{*}$ \\
\hline $\begin{array}{l}\text { Sex } \\
\text { (Male, Female) }\end{array}$ & 16.7 & 38.24 & 36.42 & $0.066 \dagger$ \\
\hline
\end{tabular}

Kruskal-Wallis test to compare the age distribution of three groups. Mann-Whitney U post hoc test Macular versus $R P, P$ 1/4 0.005; Macular versus Controls, $P<0.001$.

$+v 2$ test to compare difference in sex.

\section{Global Saturations and Diameters}

The global saturations and diameters have been summarized in Table 2. All parameters showed a significant difference between the three groups. RP group showed significantly higher saturations, lower diameters, higher AVSD and lower A-V ratios compared to both the groups. Macular dystrophies showed higher global arteriolar values and AVSD but comparable venous values to the control group. Macular and RP groups also showed statistically significant differences in the global arteriolar and venous saturations and diameters.

\section{Quadrant Saturations and Diameters}

The RP group had the highest saturations and lowest diameters in all the quadrants. The values have been summarized in Table 3.

\section{Quadrant Comparison}

The groups showed significant differences for arteriolar and venous saturation and diameter ( $p<0.001 ;<0.001$ ) for all the quadrants. Only the nasal venous saturations did not show a statistical difference.

Post hoc test with Mann-Whitney $U$ mainly showed that the RP group significantly differed from controls in all the parameters except the nasal venous saturations. Macular dystrophies differed from the controls mainly in the infero-temporal arteriolar saturations $(p<0.001)$, the global arteriolar saturation $(p=0.005)$ and the global AVSD $(p<0.001)$. Macular dystrophies and RP group differed mainly in the superior hemifield values for arteriolar values, but the venous values showed differences in both hemifields. 
TABLE 2.

Summarizing the Global Saturations and Diameters

\begin{tabular}{|c|c|c|c|c|}
\hline & (I), $n=23$ & (I), $n=29$ & $(\mathrm{Cl}), \mathrm{n}=78$ & \\
\hline $\begin{array}{l}\text { Arteriolar } \\
\text { saturation, \% }\end{array}$ & $\begin{array}{c}96.7 \\
(92.3-101.9)\end{array}$ & $\begin{array}{c}102.0 \\
(98.5-105.6)\end{array}$ & $\begin{array}{c}90.6 \\
(89.2-91.9)\end{array}$ & $<0.001 \dagger$ \\
\hline $\begin{array}{l}\text { Arteriolar } \\
\text { diameter, } \mu \mathrm{m}\end{array}$ & $\begin{array}{c}116.5 \\
(110.1-122.7)\end{array}$ & $\begin{array}{c}116.5 \\
(110.1-122.7)\end{array}$ & $\begin{array}{c}122.4 \\
(120.1-124.6)\end{array}$ & $<0.002 \ddagger$ \\
\hline $\begin{array}{l}\text { Venous } \\
\text { saturation, } \%\end{array}$ & $\begin{array}{c}54.9 \\
(51.9-58.0)\end{array}$ & $\begin{array}{c}59.1 \\
(56.4-61.8) \\
\end{array}$ & $\begin{array}{c}57.4 \\
(56.0-58.8) \\
\end{array}$ & 0.056 \\
\hline $\begin{array}{l}\text { Venous } \\
\text { diameter, } \mu \mathrm{m}\end{array}$ & $\begin{array}{c}158.4 \\
(149.8-167.1)\end{array}$ & $\begin{array}{c}148.9 \\
(141.0-156.8)\end{array}$ & $\begin{array}{c}160.4 \\
(157.4-163.4)\end{array}$ & $0.02 \S$ \\
\hline AV difference, \% & $\begin{array}{c}41.6 \\
(37.9-45.3) \\
\end{array}$ & $\begin{array}{c}43.0 \\
(39.8-46.2) \\
\end{array}$ & $\begin{array}{c}33.3 \\
(32.1-34.4) \\
\end{array}$ & $<0.001 \#$ \\
\hline AV ratiof & $\begin{array}{c}0.74 \\
(0.70-0.78)\end{array}$ & $\begin{array}{c}0.72 \\
(0.68-0.76)\end{array}$ & $0.76(0.74-0.78)$ & 0.09 \\
\hline
\end{tabular}

* Kruskal-Wallis test used to obtain significance for nonparametric data

Mann-Whitney U post hoc analysis:

tRP versus Controls $P<0.001$; Macular versus Controls $P=0.005$.

$\ddagger R P$ versus Controls $P=0.001$.

$\S R P$ versus Controls $P=0.004$.

$\triangle R P$ versus Controls $P<0.001$; Macular versus Controls $P<0.001$.

$\cap$ AV ratio implies the ratio of the diameters. 
TABLE 3.

Summarizing the Quadrantwise Arteriolar Saturations and Diameters for the Three Groups

\begin{tabular}{|c|c|c|c|c|c|}
\hline Quadrant & Parameter, n & $\begin{array}{c}\text { Macular, } \\
n=23\end{array}$ & $\begin{array}{c}R P, \\
n=62\end{array}$ & $\begin{array}{c}\text { Controls, } \\
n=78\end{array}$ & Significance* \\
\hline \multirow[t]{5}{*}{ ST } & Arteriolar saturation, $\%$ & 89.3 & 103.9 & 88.3 & $<0.001$ \\
\hline & Arteriolar diameter, $\mu \mathrm{m}$ & 127.3 & 99.9 & 132.9 & $<0.001$ \\
\hline & Venous saturation, $\%$ & 53.3 & 60.8 & 56.3 & $<0.001$ \\
\hline & Venous diameter, $\mu \mathrm{m}$ & 170.7 & 151.8 & 170.8 & $<0.001$ \\
\hline & AVSD, \% & 36.1 & 43 & 32.1 & $<0.001$ \\
\hline \multirow[t]{5}{*}{ SN } & Arteriolar saturation, $\%, 43$ & 99.4 & 106.2 & 94.5 & $<0.001$ \\
\hline & $\begin{array}{l}\text { Arteriolar diameter, } \mu \mathrm{m}, \\
43\end{array}$ & 107.9 & 93.7 & 110.3 & $<0.001$ \\
\hline & Venous saturation, \%, 61 & 57.7 & 61.0 & 60.6 & 0.306 \\
\hline & Venous diameter, $\mu \mathrm{m}, 61$ & 141.9 & 120.9 & 144.7 & $<0.001$ \\
\hline & AVSD, \%, 43 & 42.0 & 44.9 & 33.8 & $<0.001$ \\
\hline \multirow[t]{5}{*}{ IN } & Arteriolar saturation, $\%, 33$ & 99.9 & 102.5 & 93.3 & $<0.001$ \\
\hline & $\begin{array}{l}\text { Arteriolar diameter, } \mu \mathrm{m}, \\
33\end{array}$ & 102.7 & 97.7 & 111.0 & 0.001 \\
\hline & Venous saturation, \%, 59 & 57.6 & 61.1 & 60.7 & 0.166 \\
\hline & Venous diameter, $\mu$ m, 59 & 139.0 & 114.2 & 144.4 & $<0.001$ \\
\hline & AVSD, $\%, 33$ & 42.3 & 42.2 & 32.5 & $<0.001$ \\
\hline \multirow[t]{5}{*}{ IT } & Arteriolar saturation, $\%$ & 97.8 & 102.2 & 86.5 & $<0.001$ \\
\hline & Arteriolar diameter, $\mu \mathrm{m}$ & 127.2 & 106.9 & 134.7 & $<0.001$ \\
\hline & Venous saturation, $\%$ & 51.1 & 56.9 & 51.9 & $<0.001$ \\
\hline & Venous diameter, $\mu \mathrm{m}$ & 181.4 & 159.5 & 181.0 & $<0.001$ \\
\hline & AVSD, $\%$ & 46.6 & 45.2 & 34.6 & $<0.001$ \\
\hline
\end{tabular}

Number of quadrants analyzed for each parameter are given in the header row. Where the number differs from the number in the header, it has been indicated in the parameter box in italics. ST, supero-temporal, SN, supero-nasal, IN, infero-nasal, IT, infero-temporal.

* Kruskal-Wallis test used to obtain significance for nonparametric data. 
FIGURE 1.

Oximetry images with the pseudo-color maps for RP (a), macular dystrophy (b) and normal (c). Note the arteriolar attenuation and loss of analyzable vessels when threshold is set to 8 pixels.

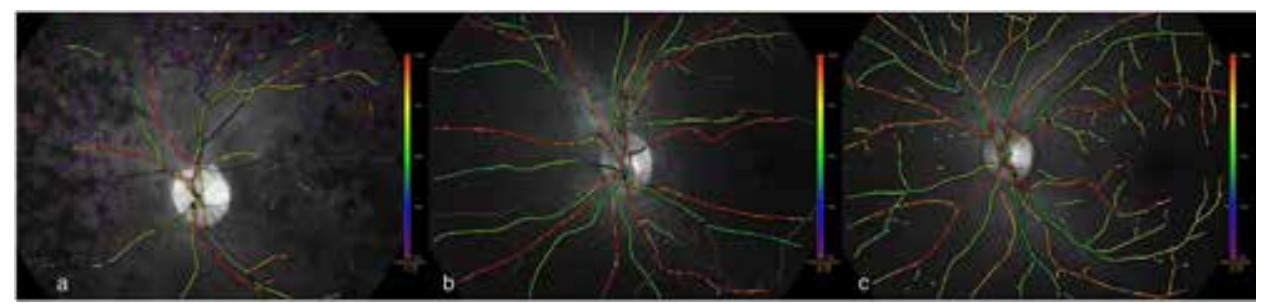

\section{Discussion}

This study represents the largest description of oxygen saturation profiles in eyes with $\mathrm{RP}$, and the first description in macular dystrophies and its comparison with RP.

A decrease in vascular diameters, an increase in arteriolar (104.1\%) and venous saturations (60.0\%) and AVSD (44.1\%) in the RP group in comparison to macular and control groups were observed in the study. Türksever at al. ${ }^{11}$ and Eysteinsson et al. ${ }^{10}$ equivocally confirm the decrease in vascular diameter. Türksever at al.11 have reported an increase in arteriolar saturation with a mean of $99.3 \%$ whereas Eysteinsson et al. ${ }^{10}$ and Ueda-Consolve et al. ${ }^{12}$ have found no change in RP with a mean of $91.7 \%$. Venous saturation was increased in all three studies (58.0\%-66.8\%). Our study was equivocal in the venous saturation increase, but found an opposite trend in the AVSD. Türksever at al. ${ }^{11}$ have mentioned that the AVSD correlated positively with the central macular thickness and have hence attributed its decrease to the progression of retinal atrophy. Eysteinsson et al. ${ }^{10}$ on the other hand have stated that both AVSD and retinal blood flow decrease in RP, and coupled with the decrease in choroidal blood flow results in decreased oxygen delivery from the retinal circulation. Both the groups indicate that the effects are secondary to the degeneration rather than primary. The alterations observed in the saturations and diameters could be a primary and resulting in the dystrophy or could be secondary to the disease process. We may hypothesize that findings that occur early in the disease are more likely primary and the ones that are seen in advanced disease may most likely be an effect of the disease process.

Macular dystrophies differed from both RP and control groups in the global arteriolar saturations, with the arteriolar saturations being highest in the RP group. Venous saturations differed from the RP group but not from controls. 
Several theories have been postulated to explain the arteriolar attenuation seen in RP. One such hypothesis is that the photoreceptor atrophy and death causes less oxygen utilization, which results in higher oxygen partial pressures in the inner retina. This would then result in a reflex constriction and reduction in ocular blood flow, thus indicating that the vascular changes are secondary to localized disease process. On the contrary, Konieckza et al. ${ }^{1}$ have stated that there is a high prevalence of PVD syndrome in RP patients, which causes alteration early in the disease. Patients with PVD syndrome react differently to stimuli such as coldness, physical or emotional stress. There is dysregulation of vessels with vasospasm. Hence a system-wide vascular spasm that also manifests in the eye could be one explanation, whereas tissue loss ${ }^{1}$, atrophy and thus a decreased demand could be the other hypothesis. The above theories may probably explain why as many as $54.8 \%$ of eyes in the RP group lacked measurable vessels in the nasal hemifield. Expectedly there was no significant difference in the vascular diameters between the macular and control groups.

There exists a capillary free zone around larger vessels that derive oxygen directly from them. ${ }^{14}$ It has been shown that translocated cells of the RPE can deposit a thick layer of extracellular matrix around retinal vessels. ${ }^{15}$ This can effectively block oxygen diffusion out of the vessels and explain the high saturation seen in the arterioles, but the unknown effect of these cells and the proximity of the measured segments to the optic disc throw enough doubt on this theory.

The thickness of the retinal nerve fiber layer (RNFL) is known to be decreased in RP. ${ }^{16,17}$ In our own study (Mohan A, Dabir S, Kummelil M, Shetty R, Kumar RS, unpublished observations, 2014), we found an inverse correlation between vascular saturation and perivascular RNFL thickness in normative Asian-Indian eyes. This could explain our observation of increasing arteriolar and venous saturation.

In a normal eye, arteriolar saturations can be expected to increase when there is more demand and less oxygen tension in the inner retinal tissue. This phenomenon, though will have to be accompanied by a corresponding increase in vascular diameters because that is the only established way the inner retina can increase oxygen delivery by physiological hyperemia. ${ }^{14}$ The arteriolar attenuation that we observed in the RP group makes this response unlikely.

An increase in arteriolar saturation can hypothetically cause a corresponding increase in venous saturation. The venous saturation can also increase due to less utilization secondary to tissue atrophy in RP. This possibly explains the increase in venous saturation seen in our patients and is similar to that noted in the other studies. 
Oxygen utilization by tissue is the product of AVSD and blood flow. ${ }^{14}$ Blood flow is heavily dependent on the vascular diameters. It is known that the A-V transit time is increased and vascular diameters are decreased in RP, thus resulting in very low volume of blood flow. Hence, for a given amount of oxygen extraction, the AVSD would increase if the blood flow decreases. This is accompanied by a decreased choroidal blood flow in RP. ${ }^{8}$ We could possibly imply that even though tissue atrophy and death would cause lower oxygen demand, in view of the fall in blood flow, AVSD would have to increase to adequately meet the demands of the residual functioning retina. In addition, hypoxic tissue would extract more oxygen per unit volume of blood. ${ }^{10}$ This could possibly explain the increased AVSD seen in the RP group in our study.

An alteration in oxygen saturation profiles was seen in all quadrants in the RP group but mainly in the infero-temporal quadrant in the macular group. The lower saturations in the infero-temporal venules in normative individuals has been attributed to its anatomic location in relationship to the optic disc. ${ }^{18}$ In macular dystrophies, it stands to reason that the macular blood supply is the most affected compared to those with RP, thus contributing to the altered oxygen saturation profiles that we noted in the inferotemporal quadrant.

Our study is limited by the relatively small sample size we had in the macular dystrophy group. Another limitation was that the blood pressure was not measured, but it is assumed that there was no difference in blood pressure between those with retinal degeneration and age-matched controls, given the relatively young age of the subjects included in the study. The current approach of our study excludes vessels narrower than 8 pixels or 74 micrometer, hence we cannot comment on vessels that were narrower than this cutoff and therefore not measurable. A correlation of oxygen saturation profiles with RNFL thickness could throw further light on the reasons for our observations. A correlation with electro-retinographic findings can in the future help us obtain a better correlation between oximetry values and disease severity.

This study represents the largest so far reported on retinal dystrophies and establishes the increase in arteriolar, venous saturations and AVSD increase. It is important to ascertain whether the hemodynamic observations are primary or secondary. If they are secondary, they will only help us establish severity; if primary they have a multitude of therapeutic implications. It has also been shown that the central defects progress slower with nilvadipine which is a calcium channel blocker. ${ }^{19}$ Magnesium, omega-3 fatty acids and fludrocortisone have all been hypothesized to help in the treatment of RP by altering the hemodynamic parameters. ${ }^{1}$ If the above methods prove to be effective, 
retinal oximetry can be used as a non-invasive tool to identify potential patients who might benefit and subsequently monitor response to treatment.

\section{References}

1. Konieczka K, Flammer AJ, Todorova M, et al. Retinitis pigmentosa and ocular blood flow. EPMA J 2012;3:17.

2. Chizzolini M, Galan A, Milan E, et al. Good epidemiologic practice in retinitis pigmentosa: from phenotyping to biobanking. Curr Genomics 2011;12:260-266.

3. Bunker $\mathrm{CH}$, Berson EL, Bromley WC, et al. Prevalence of retinitis pigmentosa in Maine. Am J Ophthalmol 1984;97:357-365.

4. Sen $P$, Bhargava A, George $R$, et al. Prevalence of retinitis pigmentosa in South Indian population aged above 40 years. Ophthalmic Epidemiol 2008;15:279-281.

5. Roosing S, Thiadens AAHJ, Hoyng CB, et al. Causes and consequences of inherited cone disorders. Progress in Retinal and Eye Research 2014;42C:1-26.

6. Komeima K, Rogers BS, Campochiaro PA. Antioxidants slow photoreceptor cell death in mouse models of retinitis pigmentosa. J Cell Physiol 2007;213:809-815.

7. Wolf S, Pöstgens $H$, Bertram B, et al. Hemodynamic findings in patients with retinitis pigmentosa. Klin Monbl Augenheilkd 1991;199:325-329.

8. Cellini M, Strobbe E, Gizzi C, Campos EC. ET-1 plasma levels and ocular blood flow in retinitis pigmentosa. Can J Physiol Pharmacol 2010;88:630-635.

9. Hardarson $\mathrm{SH}$, Harris A, Karlsson RA, et al. Automatic Retinal Oximetry. Invest Ophthalmol Vis Sci 2006;47:5011-5016.

10. Eysteinsson T, Hardarson SH, Bragason D, Stefánsson E. Retinal vessel oxygen saturation and vessel diameter in retinitis pigmentosa. Acta Ophthalmologica 2014;92:449-453.

11. Türksever C, Valmaggia C, Orgul S, et al. Retinal vessel oxygen saturation and its correlation with structural changes in retinitis pigmentosa. Acta Ophthalmologica 2014;92:454-460.

12. Ueda-Consolvo T, Fuchizawa C, Otsuka M, et al. Analysis of retinal vessels in eyes with retinitis pigmentosa by retinal oximeter. Acta Ophthalmol.

13. Geirsdottir A, Palsson O, Hardarson SH, et al. Retinal Vessel Oxygen Saturation in Healthy Individuals. Invest Ophthalmol Vis Sci 2012;53:5433-5442.

14. Kur J, Newman EA, Chan-Ling T. Cellular and physiological mechanisms underlying blood flow regulation in the retina and the choroid in health and disease. Prog Retin Eye Res. 2012; 102: 805-816 
15. Li ZY, Possin DE, Milam AH. Histopathology of bone spicule pigmentation in retinitis pigmentosa. Ophthalmology 1995;102:805-816.

16. Oishi A, Ogino K, Nakagawa S, et al. Longitudinal analysis of the peripapillary retinal nerve fiber layer thinning in patients with retinitis pigmentosa. Eye 2013;27:597-604.

17. Garcia-Martin E, Pinilla I, Sancho E, et al. Optical coherence tomography in retinitis pigmentosa: reproducibility and capacity to detect macular and retinal nerve fiber layer thickness alterations. Retina (Philadelphia, Pa) 2012;32:1581-1591.

18. Jani PD, Mwanza J-C, Billow KB, et al. Normative values and predictors of retinal oxygen saturation. Retina (Philadelphia, Pa) 2014;34:394-401.

19. Nakazawa $M$, Ohguro $H$, Takeuchi $K$, et al. Effect of nilvadipine on central visual field in retinitis pigmentosa: a 30-month clinical trial. Ophthalmologica 2011;225:120-126. 



\section{IDENTIFICATION OF NOVEL MUTATIONS IN ABCA4 GENE: CLINICAL AND GENETIC ANALYSIS OF INDIAN PATIENTS WITH STARGARDT DISEASE}

Rajani Battu, Anshuman Verma, Ramesh Hariharan,

Shuba Krishna, Ravi Kiran, Jemima Jacob, Aparna Ganapathy, Vedam L. Ramprasad, Govindasamy Kumaramanickavel, Nallathambi Jeyabalan, Arkasubhra Ghosh BIOMED RESEARCH INTERNATIONAL 2015:2015; 1-10 



\section{Abstract}

Stargardt disease (STGD) is the leading cause of juvenile macular degeneration associated with progressive central vision loss, photophobia and color vision abnormalities. In this study, we have described the clinical and genetic features of Stargardt patients from an Indian cohort. Next-generation sequencing was carried out in five clinically confirmed unrelated patients and their family members using a gene panel comprising 184 specific genes. Sequencing results were analyzed by read mapping and variant calling in genes of interest, followed by their verification and interpretation. Genetic analysis revealed ABCA4 mutations in all of the five unrelated patients. Among these four patients were found with compound heterozygous mutations and other one had homozygous mutation. All the affected individuals showed signs and symptoms consistent with the disease phenotype. We report two novel ABCA4 mutations in Indian patients with STGD disease, which expands the existing spectrum of disease-causing variants and the understanding of phenotypic and genotypic correlations. Screening for causative mutations in patients with STGD using panel of targeted gene sequencing by NGS would be a cost effective tool, might be helpful in confirming the precise diagnosis, and contributes towards the genetic counseling of asymptomatic carriers and isolated patients. 


\section{Introduction}

Stargardt disease (STGD: OMIM \#248200/\#600110) is an inherited genetic eye disease in which patients develop bilateral macular dystrophy leading to progressive loss of central vision in early childhood. It is the most common form of autosomal recessive juvenile macular dystrophy with a reported prevalence of 1:10000., ${ }^{1,2}$ The disease is characterized by loss of central vision, fundus flavimaculatus, mottling or atrophy of the retinal pigment epithelium (RPE), bull's eye maculopathy, flecks in the macula, beaten-bronze macular appearance and cone-rod dysfunction. ${ }^{3}$ STGD is associated with accumulation of lipofuscin content in RPE cells, failure in removal of toxic substances and significant photoreceptor cell death. ${ }^{4-6}$ So far, mutations have been reported in six candidate genes (ABCA4, ELOVL4, PROM1, PRPH2, and CRB1) in various forms of panretinal dystrophies with the possible phenotype of STGD1. ${ }^{7-}$

12 Mutations in ABCA4 gene are implicated in recessive STGD type I. ${ }^{13,14}$ ABCA4 is a large gene which consists of 50 exons located in chromosome $1 \mathrm{p} 13 .{ }^{15} \mathrm{It}$ is a member of the subfamily A of the ATP-binding cassette $(A B C)$ transporters that is expressed in the retinal outer segments of cone and rod photoreceptor cells. This gene is involved in the transport and clearance of all-trans-retinal aldehyde, a by-product of the retinoid cycle of vision, and other essential molecules across the disc membrane into the cytoplasm. ${ }^{16-18}$ Defective ABCA4 leads to an accumulation of retinoids in the outer segment or the retinal pigment epithelium and non-homogenous slowing of retinoid cycle kinetics, as observed in earlier studies in STGD patients. ${ }^{13}$ In the animal model, ABCA4-knockout mice show deposition of lipofuscin-like substance in the RPE. Additionally, increased levels of lipofuscin components such as fluorescent diretinoid, A2E, diretinalpyridinium are also observed. ${ }^{19,20}$ Interestingly, knockout of RDH8 (retinol dehydrogenase 8 ) which conducts all-trans-retinal aldehyde metabolism together with $A B C A 4$ showed the retinal degeneration in mice. ${ }^{21}$ Moreover, aged ABCA4 knockout albino mice display a mild retinal degeneration. ${ }^{22}$ It is well known that ABCA4 mutations are heterogeneous and cause various forms of retinal dystrophies, which includes STGD, retinitis pigmentosa, age-related macular degeneration (AMD) and cone-rod dystrophy. ${ }^{12,23-26}$ In this study, we describe novel and reported ABCA4 gene variants and associated phenotypes in Indian patients with STGD. 


\section{Methods}

\section{Study Subjects and Clinical Ascertainment}

Five clinically confirmed unrelated patients with Stargardt disease and their available family members were enrolled for genetic analysis. Study subjects underwent a complete ophthalmic examination including measurement of visual acuity, intraocular pressure, slit lamp biomicroscopy, detailed fundus examination, fundus photography, fundus autofluorescence (FAF), spectral domain optical coherence tomography (SD$\mathrm{OCT}$ ) and full field electroretinography (ERG). Fifty normal controls without any history of eye diseases were included. This study adhered to the tenets of Declaration of Helsinki and was approved by the institutional ethical committee (IEC). Informed consent was obtained from all subjects for the study and publication of their data.

\section{DNA Extraction}

Five $\mathrm{ml}$ of peripheral blood samples were obtained from all subjects in EDTA coated vacutainers. Total genomic DNA was extracted using the salt precipitation method described previously. ${ }^{27}$ Agarose gel electrophoresis was done to examine the integrity of genomic DNA.

\section{Sample Preparation}

Fifty nanogram of Qubit quantified DNA was used for library preparation using the Illumina Nextera protocol as per the manufacturer's instructions. This protocol uses transposon-based shearing of genomic DNA and allows the DNA to be "tagmented", i.e., fragmented and tagged simultaneously in the same tube. Limited cycle PCR was carried out to incorporate adaptors and sample specific barcodes to prepare the sample library. The tagged and amplified sample library was checked for quality using BioAnalyzer (Agilent, USA) and quantified.

\section{Target Enrichment and Exome Sequencing}

Approximately 500 nanogram of library was used for enrichment, involving two successive hybridization steps with target specific biotinylated probes. These probes were targeted at the exons of 184 genes with previously known pathogenic variations associated with multiple eye disorders. Bound DNA was pulled down using streptavidin beads. The target library was amplified using limited cycles of PCR. 6-10 pM of the enriched library was loaded for sequencing on to a MiSeq sequencer (Illumina, USA) 
using a standard v2 kit.

\section{Data Analysis}

ALIGNMENT: The trimmed fastq files were generated using MiSeq Reporter from Illumina. The reads were aligned against the whole genome hg19 build using Strand NGS v1.6 (Strand Life Sciences) after trimming at least $1 \mathrm{bp}$ at the $3^{\prime}$ end and any others with quality below 10 . Reads, which had length less than $25 \mathrm{bp}$ after trimming, were not considered for alignment. A maximum of 5 matches of alignment score at least $90 \%$ were computed. The reads were realigned using the local realignment tool in Strand NGS. Reads that failed vendor QC, reads with average quality less than 20, reads with ambiguous characters, and translocated and single-mate flip reads were all filtered out.

VARIANT CALLING: The Strand NGS variant caller was used to detect variants at locations in the target regions covered by a minimum of 10 reads with at least 2 variants reads. Variants with a decibel score of at least 50 were reported. Results were further compared with the dbSNP138 to identify novel variations. The potential deleterious effect of variants were determined by using various insilico prediction algorithms (PolyPhen, SIFT, Mutation Taster. PhyloP, LRT GERP++RS).

VARIANT INTERPRETATION: Variants were then imported into Strandomics v1.0 (Strand Life Sciences) for annotation, prioritization, and reporting based on ACMG guidelines. Six known genes (ABCA4, CNGB3, CRB1, ELOVL4, PROM1, PRPH2) for STGD were included for interpretation.

\section{Validation of ABCA4 mutations}

The potential ABCA4 variations were revalidated in all the affected individuals and in the available unaffected family members as well as 50 unrelated normal controls using polymerase chain reaction (PCR) and Sanger sequencing. ABCA4 coding exons $(5,17,19,35,42,46$, and 47$)$ and their flanking splice junctions were PCR amplified using the primers reported previously. ${ }^{28}$ PCR amplicons were sequenced on a $3730 \mathrm{xl}$ DNA Sequencing Analyzer (Life Technologies). Sequencing results were analyzed in FinchTV software (Geospiza, Seattle, WA, USA) and compared with the reference databases (NM_000350, ENSEMBL, and ENST00000370225). 


\section{Results}

Genetic analysis was performed on a group of five unrelated patients with clinical findings of STGD (Figure 1). In this study, we performed NGS analysis in a panel of 184 genes encompassing multiple eye disorders including Stargardt disease. However the data analysis was performed for six known Stargardt related genes (ABCA4, ELOVL4, CNGB3, PROM1, PRPH2, and CRB1). This genetic analysis revealed a total of five mutations in the ABCA4 gene. One of these was a previously reported homozygous mutation (p.Arg2149X) with two asymptomatic heterozygous carriers in a five generation consanguineous family. Two novel heterozygous mutations, p. Phe191Valfs42 and p.Tyr872X, were identified in two different patients along with previously reported heterozygous mutations (p.Gly172Ser, p.Gly1961Glu, resp.), resulting in a compound heterozygous state (p.Phe191Valfs42/p.Gly172Ser and p.Tyr872X/p.Gly1961Glu). In addition, previously reported compound heterozygous mutations (p.Arg1640Trp/p. Gly1961Glu) and a previously reported homozygous mutation (p.Thr971Asn) were found in two isolated cases. There are no pathogenic mutations that were identified in other candidate genes associated with STGD. The clinical features of the patients are described in Table 1. We also found nonpathogenic reported polymorphisms (SNPs) in the Stargardt related genes (ABCA4, ELOVL4, CNGB3, PROM1, PRPH2, and CRB1), which are listed in Table 2. 
FIGURE 1.

(a): In Family SG-01 patient II:1 had a (p.Arg1640Trp) and (p.Gly1961Glu) compound heterozygous mutation. (b): In Family SG-02, the patient_II:1 had novel (p.Phe191Valfs42) and reported p.Gly172Ser mutation. (c): In Family SG03, the affected individual had a homozygous mutation ( $\mathrm{T}$.Thr971Asn) mutation. (d): Patient (II:1) from family SG04_Il:1 had a novel stop codon (p.Tyr872X) and (p.Gly1961Glu) mutation.(e): Patient (V:1) from a five-generation Indian family (SG-05) had a reported homozygous mutation (p.Arg2149X) in ABCA4. The asterisk denotes the individuals were included for the genetic analysis. Arrows indicate the proband in each family.

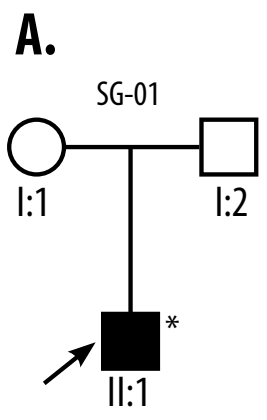

Arg1640Trp/Gly1961Glu
B.

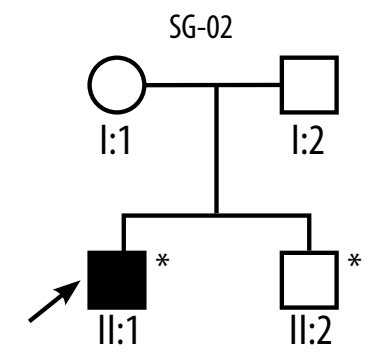

Phe191Valfs42/Gly172 Ser+/Gly172Ser
C. SG-03

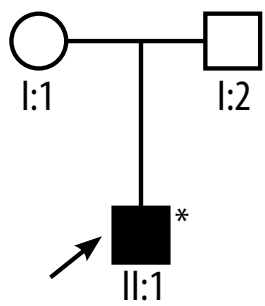

Thr971Asn/Thr971Asn

E. SG-05

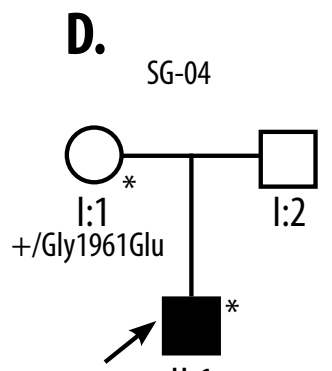

II:1

Tyr872X/Gly1961Glu

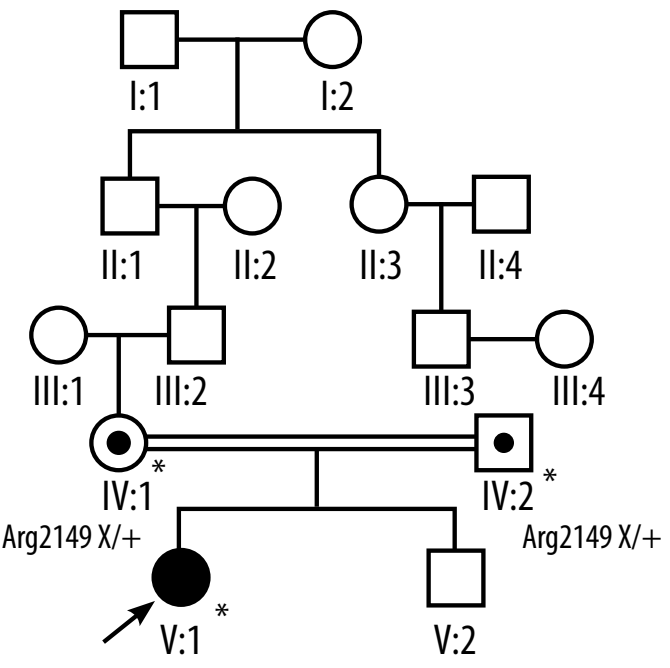

Arg2149 X/Arg2149X 
TABLE 1.

Clinical features of STGD patients with ABCA4 mutations

\begin{tabular}{|c|c|c|c|c|c|c|c|c|c|}
\hline \multirow{2}{*}{ Family Id } & \multirow{2}{*}{ Age/Sex } & \multicolumn{2}{|c|}{ BCVA } & \multicolumn{2}{|c|}{ Refraction } & \multirow{2}{*}{ Fundus } & \multirow{2}{*}{ FAF } & \multirow{2}{*}{ SD-OCT } & \multirow{2}{*}{$\begin{array}{l}\text { Full-field } \\
\text { ERG }\end{array}$} \\
\hline & & RE & LE & RE & LE & & & & \\
\hline SG-01_Il:1 & $27 / M$ & $\begin{array}{c}6 / 60 \\
\text { N8 }\end{array}$ & $\begin{array}{c}6 / 36 \\
\text { N8 }\end{array}$ & $-2.5 \mathrm{DS}$ & $-2.75 \mathrm{DS}$ & $\begin{array}{l}\text { Macular atrophy, } \\
\text { No flecks }\end{array}$ & $\begin{array}{l}\text { Hypo AF } \\
\text { surrounded } \\
\text { by ring of } \\
\text { hyper AF }\end{array}$ & $\begin{array}{l}\text { IS/OS } \\
\text { loss }\end{array}$ & Normal \\
\hline SG-02_II:1 & $31 / M$ & $\begin{array}{c}6 / 60 \\
\text { N34 }\end{array}$ & $\begin{array}{l}4 / 60 \\
\text { N36 }\end{array}$ & 0 & 0 & $\begin{array}{c}\text { Macular atrophy, } \\
\text { macular flecks }\end{array}$ & $\begin{array}{l}\text { Hypo AF } \\
\text { surrounded } \\
\text { by hyper } \\
\text { and hypo } \\
\text { AF flecks }\end{array}$ & $\begin{array}{l}\text { IS/OS } \\
\text { loss }\end{array}$ & Normal \\
\hline SG-03_Ill:1 & $22 / \mathrm{M}$ & $\begin{array}{c}6 / 60 \\
N 18\end{array}$ & $\begin{array}{l}6 / 45, \\
\text { N18 }\end{array}$ & $\begin{array}{c}-1.25 \mathrm{DS} / \\
-1.00 \mathrm{DC} \\
900\end{array}$ & $\begin{array}{l}-1.50 \text { DS/ } \\
-0.50 \text { DC } 200\end{array}$ & $\begin{array}{c}\text { Macular atrophy, } \\
\text { extensive flecks }\end{array}$ & $\begin{array}{l}\text { Hypo AF } \\
\text { surrounded } \\
\text { by hyper } \\
\text { and hypo } \\
\text { AF flecks }\end{array}$ & $\begin{array}{l}\text { IS/OS } \\
\text { loss }\end{array}$ & $\begin{array}{l}\text { Rod-cone } \\
\text { dysfunction }\end{array}$ \\
\hline SG-04_II:1 & $26 / M$ & $\begin{array}{l}6 / 38 \\
N 12\end{array}$ & $\begin{array}{l}6 / 38 \\
\text { N12 }\end{array}$ & $\begin{array}{l}-1.00 \text { DS/- } \\
0.5 \text { DC } 700\end{array}$ & $-1.50 \mathrm{DS}$ & $\begin{array}{l}\text { Macular atrophy, } \\
\text { No flecks }\end{array}$ & $\begin{array}{c}\text { Hypo AF } \\
\text { surrounded }\end{array}$ & $\begin{array}{l}\text { IS/OS } \\
\text { loss }\end{array}$ & Normal \\
\hline SG-05_V:1 & $16 / F$ & $\begin{array}{l}6 / 60, \\
\text { N12 }\end{array}$ & $\begin{array}{l}\text { 6/38, } \\
\text { N12 }\end{array}$ & $\begin{array}{l}+0.5 \mathrm{DS} /- \\
0.75 \mathrm{DC} 50\end{array}$ & $\begin{array}{c}+0.5 \text { DS/- } \\
0.75 \text { DC } 1700\end{array}$ & $\begin{array}{l}\text { Macular atrophy, } \\
\text { temporal pallor of } \\
\text { optic disc }\end{array}$ & $\begin{array}{c}\text { Hypo AF } \\
\text { surrounded } \\
\text { by hyper AF } \\
\text { flecks }\end{array}$ & $\begin{array}{l}\text { IS/OS } \\
\text { loss }\end{array}$ & $\begin{array}{l}\text { Cone-rod } \\
\text { dysfunction }\end{array}$ \\
\hline
\end{tabular}

BCVA: best corrected visual acuity, RE: right eye, LE:left eye, BE: both eyes, IOP: intraocular pressure, PR: photoreceptors, AF: autofluorescent, SD-OCT: Spectral domain optical coherence tomography, FAF: fundus autofluorescence, ERG: electroretinography. IS/OS: inner segment/outer segment layer, NA: Not available 
TABLE 2.

List of nonpathogenic variations identified in patients with STGD by NGS analysis.

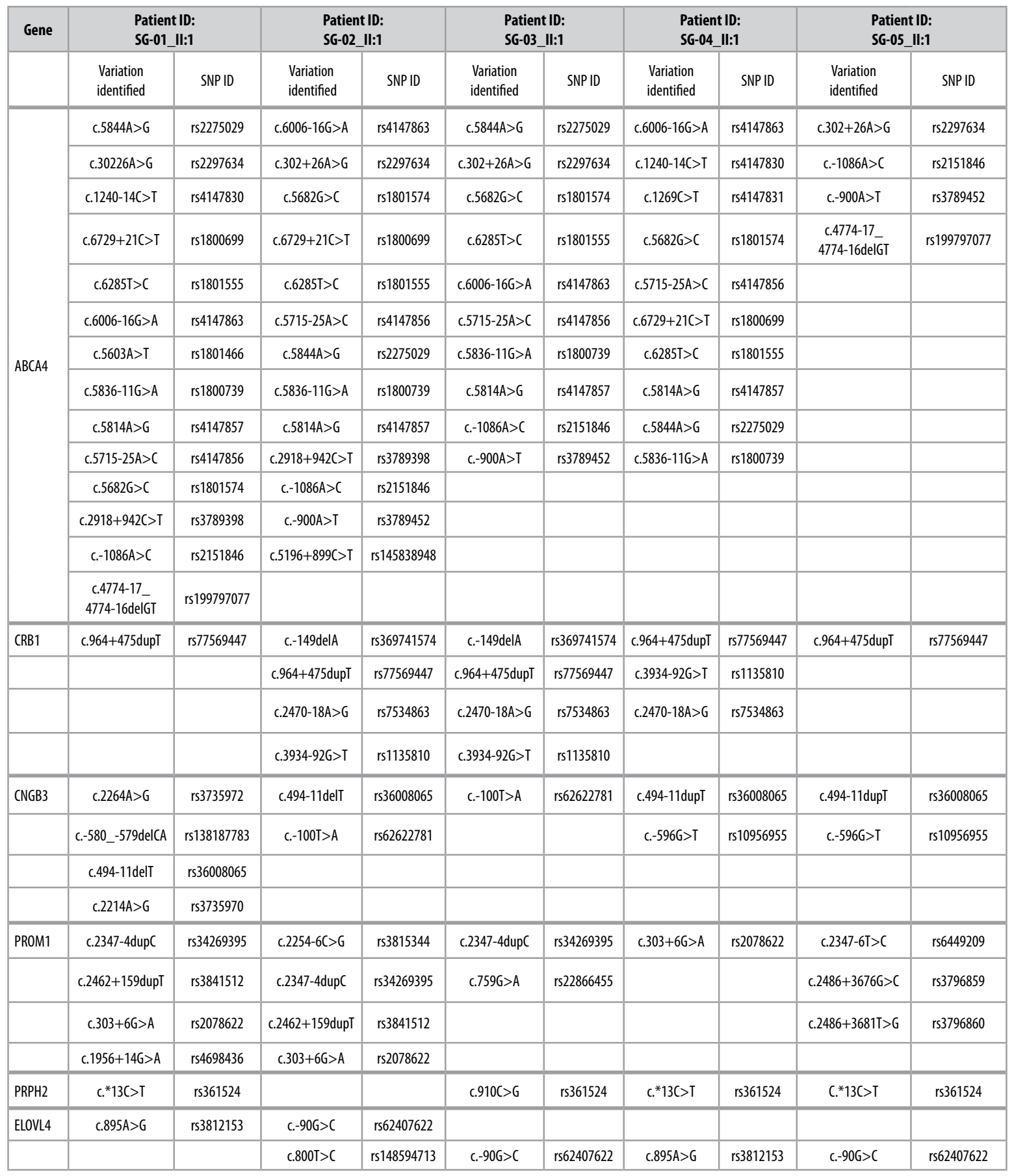


FIGURE 2.

Clinical features of Stargardt patients with ABCA4 mutations

A. SG-01_Il:1

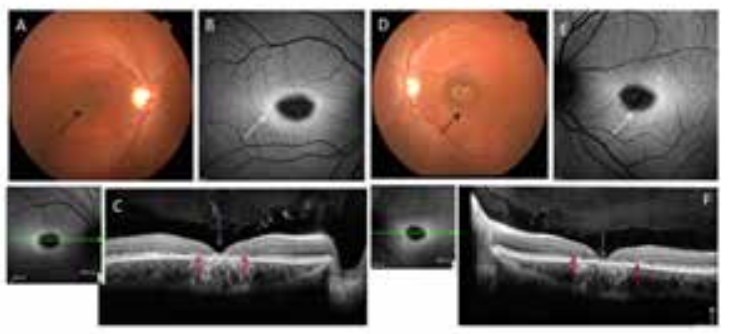

A. (SG-01_II:1): A \& D show the fundus photos of the right and left eye. Note the atrophic macular lesions at the macula (black arrows). B \& Eshow the corresponding fundus autofluorescence. Note the central area of hypo-autofluorescence with a surrounding ring of hyper-autofluorescence (white arrows). (\& F shows the foveal thinning (blue arrows) and the loss of photoreceptors and the external limiting membrane (red arrows).

B. SG-02_II:1

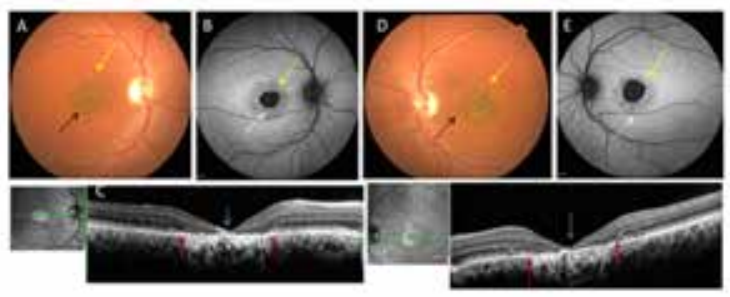

B. (SG-02_II:1): A \& D show the fundus photos of the right and left eye. Note the atrophic lesions at the macula (black arrows) and the flecks (yellow arrows). $B \&$ Eshow the corresponding fundus autofluorescence. Note the central area of hypo-autofluorescence with a surrounding ring of hyper-autofluorescence (white arrows). The yellow arrow corresponds to the flecks on FAF. (\& F shows the foveal thinning (blue arrows) and the loss of photoreceptors and the external limiting membrane (red arrows).

C. SG-03_Il:1

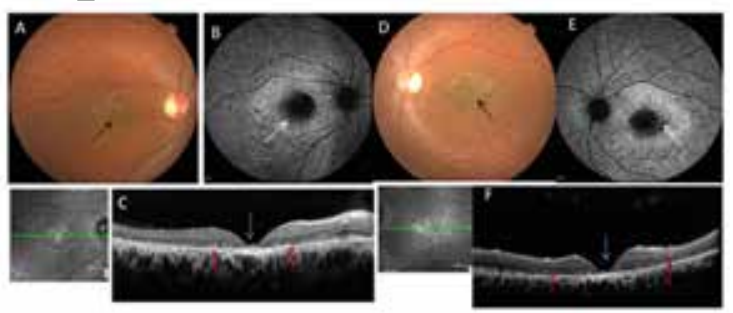

C. (SG-03_II:1): A \& D show the fundus photo of the right and left eye. Note the central atrophic macula (black arrows) and the extensive flecks seen throughout the posterior pole. $B \& E$ - FAF shows the corresponding area of central hypo-autofluorescence (white arrow) surrounded by an area of hyperautofluorescence. Note the hyper and hypo autofluorescence of flecks on FAF. ( \& ESD-OCT shows the central foveal thinning (blue arrow) and the loss of photoreceptors centrally (red arrows).

D. SG-04_II:1

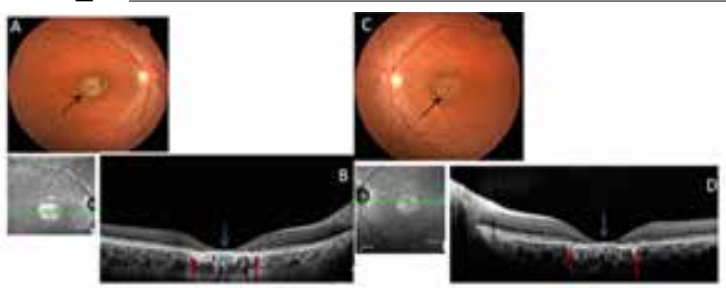

D. (SG-04_II:1): A \& C show the fundus photos of the right and left eye. Note the atrophic lesions at the macula (black arrows). B \& D shows the foveal thinning (blue arrows) and the loss of photoreceptors and the external limiting membrane (red arrows)

E. SG-05_II:1

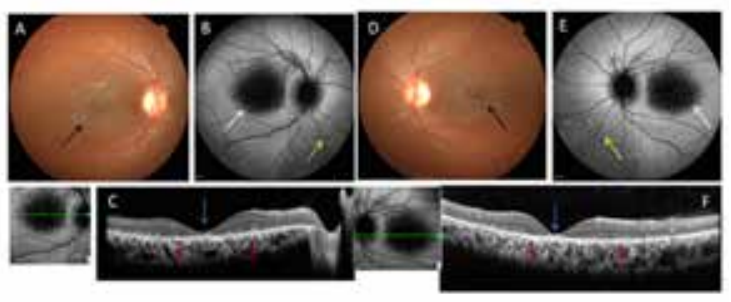

E. (SG-04_V:1): A \& D show the fundus photos of the right and left eye. Note the central atrophic macula (black arrows) and the extensive flecks seen throughout the posterior pole. $B \& E$ - FAF shows the corresponding area of central hypo-autofluorescence (white arrow). Note the hyper-autofluorescencent flecks (yellow arrows) that are not clear on the fundus pictures. (\&ESD-OCT shows the central foveal thinning (blue arrow) and the extensive loss of photoreceptors (red arrows). 
FIGURE 3.

Sequencing analysis of two patients with novel ABCA4 mutations

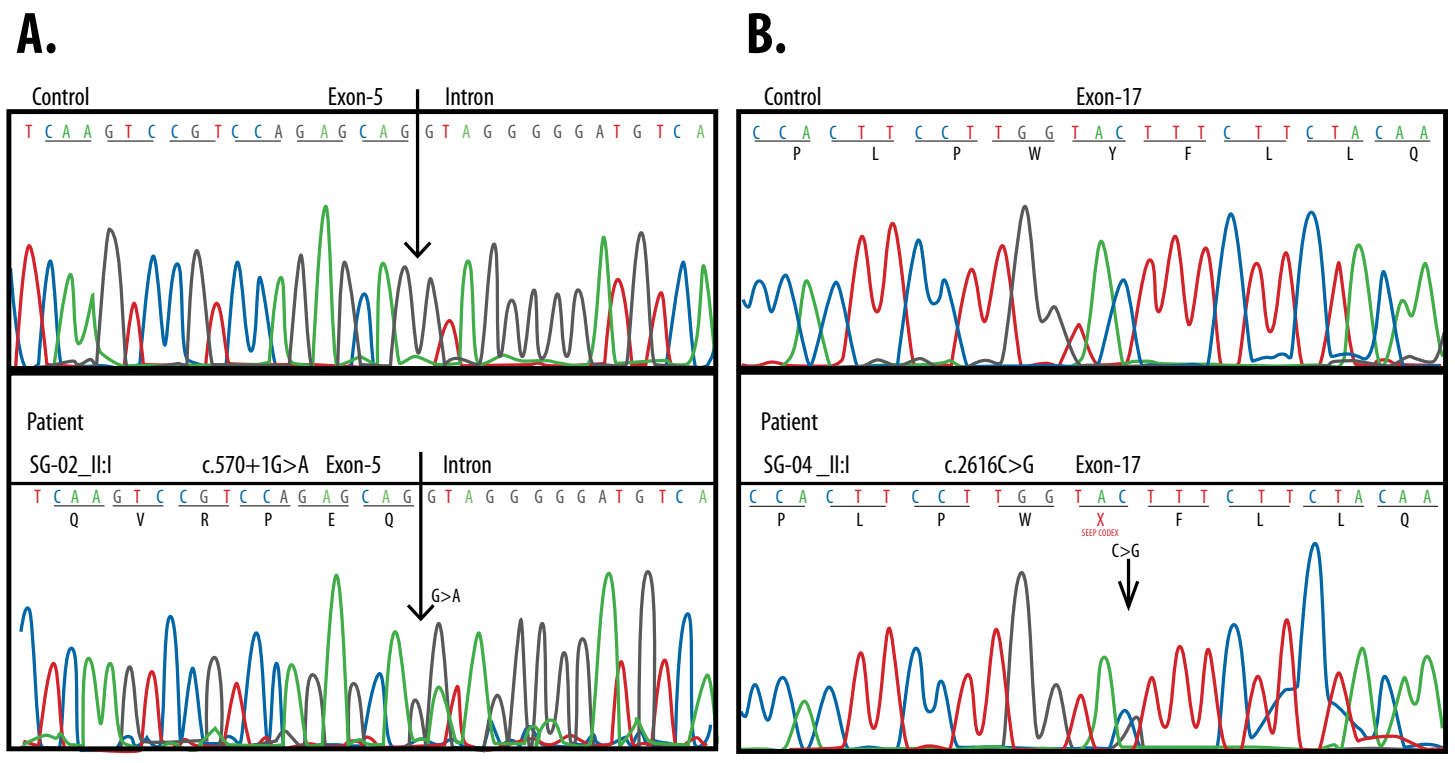

A and B show the comparison of sequence chromatograms of two patients (SG-02 II:1, SG-04_II:1) and the normal controls. Arrows indicate the positions of nucleotides change

3.1 Family: SG-01.Patient (II:1) was a 27-year old male from non-consanguineous parents with progressive decrease of vision in both eyes (Figure 1(a)). His bestcorrected visual acuity was $6 / 60,6 / 36$ in the right eye (RE) and left eye (LE) respectively. Anterior segment examination and the intra ocular pressure (IOP) in both eyes were normal. Fundus showed an appearance of bull's eye maculopathy in both eyes. There were no visible flecks. Fundus autofluorescence (FAF) showed an area of hypoautofluorescence corresponding to the atrophic macula surrounded by a ring of hyperautofluorescence (Figure 2(a)). The ring of hyperautofluorescence corresponds to the lipofuscin-laden RPE cells while the central hypofluorescent area corresponds to an area where there is complete RPE atrophy. Full field electroretinography of both eyes was normal suggestive of group 1 STGD. SD-OCT of both eyes showed a central area of photoreceptor loss corresponding to the atrophy seen on the fundus and FAF (Figure 2(a)). Genetic analysis in patient (II:1) revealed two previously reported missense mutations, c.4918C >T (p.Arg1640Trp) and c.5882G>A (p.Gly1961Glu), in compound heterozygous state in the ABCA4 gene. These compound heterozygous missense mutations were not present in 50 unrelated normal controls. The parents $(\mathrm{I}: 1, \mathrm{I}: 2)$ were clinically normal and did not consent to genetic analysis 
3.2 Family: SG-02 Patient (II:1) in the second family was a 31-year old male with complaints of blurred vision for distance since the age of 20 years. He was the first sibling of non-consanguineous parents (Figure 1(b)). His BCVA was 6/60, N24 in the $\mathrm{RE}$ and 4/60, N36 in the LE respectively. Fundus examination of both eyes showed a central atrophic lesion with flecks restricted to the macula (Figure 2(b)). Anterior segment examination and the IOP were normal in both eyes. The central lesion was hypoautofluorescent on FAF corresponding to the area of RPE atrophy surrounded by hypo- and hyperfluorescent flecks. SD-OCT showed an area of photoreceptor loss corresponding to macular atrophy (Figure 2(b)). Full field ERG of both eyes were normal suggestive of group 1 STGD. Genetic analysis showed two mutations c.570+1G>A and c.514G>A (p.Gly172Ser) in compound heterozygote state. These mutations were not observed in 50 unrelated controls. The nucleotide change c.570+1G $>A$ was a novel essential splice site mutation predicted to result in a frameshift at Phe 191. As a result, there would be a replacement of phenylalanine with valine followed by termination after 42 amino acids (p.Phe191Valfs42) (Figure3 (a)). The proband's parents were clinically normal and not tested for this mutation. The proband's brother (II:2) was normal on ophthalmic examination. Genetic screening revealed he was carrying only the p.Gly172Ser mutation but missing the essential splice site mutation c.570+1G>A.

3.3 Family: SG-03 The patient (II:1) in the third family was a 22-year old male who presented with difficulties in night vision and blurred vision for distance since the age of 10 . He was the only child of nonconsanguineous parents (Figure1 (c)). His BCVA was $6 / 60, N 18$ in the RE and 6/45, N18 in the LE respectively. Slit-lamp examination of the anterior segment revealed no abnormalities in both eyes with normal IOP. Fundus examination of both eyes showed an atrophic macula with retinal flecks (Figure2 (c)). FAF showed a central area of hypoautofluorescence corresponding to the atrophic macula and areas of multiple hyperautofluorescence corresponding to the flecks. SDOCT of both eyes showed foveal thinning with extensive loss of photoreceptors (Figure2 (c)). The full field ERG of both eyes showed rod-cone dysfunction suggestive of group 3 STGD. Genetic analysis of patient (II:1) showed a previously reported homozygous mutation c.2912C>A (p.Thr971Asn) in ABCA4 gene that was not present in 50 unrelated healthy controls analyzed. His parents were normal on ophthalmic examination. We were unable to examine the segregation of this mutation in the parents.

3.4 Family: SG-04 The patient (II:1) in the fourth family was a 26-year old male with loss of central vision. He was the only child of nonconsanguineous parents (Figure $1(d)$ ). His BCVA was 6/38, N12 in both eyes. Fundus of both eyes showed atrophic macular lesions 
(Figure 2(d)). Anterior segment examination and the IOP in both eyes were normal. There were no flecks, FAF showed central hypoautofluorescence corresponding to the central macular lesion. SD-OCT showed central loss of photoreceptors corresponding to the macular atrophy (Figure 2(d)). Full field ERG of both eyes was normal suggestive of group 1 disease. Genetic analysis of patient (II:1) revealed a novel mutation c.2616C>G (p.Tyr872X) and a previously reported mutation c.5882G >A (p.Gly1961Glu) occurring in a compound heterozygous state in the ABCA4 gene (Figure 3(b)). His unaffected mother (I:1) harbored only one heterozygous variant (p.Gly1961Glu) in ABCA4. His father (I:2) was clinically normal and was not included for genetic testing. These compound heterozygous mutations were not present in the 50 normal controls.

3.5 Family: SG-05 We identified a 12-year old girl (V:1) from a five-generation Indian family with the characteristic features of STGD. (Figure 1(e)). She presented with inability to see the letters on the classroom board since the age of 8 years. She was the first child of a consanguineous marriage. Her BCVA was 6/60, N12 and 6/38, N12 in the RE and LE respectively on the Snellen's chart. Slit lamp examination showed normal anterior segment and IOP in both eyes. Fundus examination of both eyes showed mild temporal pallor of the optic nerves with an atrophic macula (Figure 2(e)). FAF of both eyes showed an area of hypoautofluorescence corresponding to the atrophic macula with multiple hyperautofluorescent areas corresponding to flecks. SD-OCT of both eyes showed foveal thinning with extensive photoreceptor loss. Full field ERG in both eyes showed a cone-rod dysfunction suggestive of group 2 STGD (Figure 2(e)). The parents and sibling were clinically normal. Genetic analysis of the patient ( $\mathrm{V}: 1)$ showed a previously reported homozygous change c.6445C>T (p.Arg2149X) in the ABCA4 gene. Her unaffected parents (IV:1, IV:2) were both heterozygous for the same mutation (p.Arg2149X) showing an asymptomatic carrier status. This mutation was not present in 50 unrelated controls.

\section{Discussion}

Mutational screening for Stargardt patients has not been described in detail in Indian patients earlier. An appreciation of the genetic basis is important for understanding the biology of the disease. In this study, we report five mutations in the ABCA4 gene, two of which have not been previously reported, and we determine the clinical phenotype associated with these genotypes in five unrelated patients with STGD. Mutations in ABCA4 gene are the most common causes of STGD in inherited as well as sporadic cases. Several pathogenic mutations in ABCA4 have been reported in various ethnic 
backgrounds of STGD and documented in the HGMD (http://www.biobase-international. com/product/hgmd) and dbSNP databases (http://www.ncbi.nlm.nih.gov/SNP/). Apart from STGD, ABCA4 mutations have also been reported in other ocular disorders including cone-rod dystrophy, ${ }_{1}^{29}$ and autosomal recessive retinitis pigmentosa. ${ }^{25}$

The patient (SG-01_Il:1) carries two pathogenic missense mutations p.Gly1961Glu and p.Arg1640Trp, both well-known disease-causing mutations in STGD. p.Gly1961Glu has been reported most frequently in Caucasian and Spanish patients with STGD, ${ }^{30,31}$ in 11 probands who exhibited compound heterozygosity with other missense variations in ABCA4. ${ }^{17}$ p.Arg1640Trp was identified in a compound heterozygous Stargardt patient who harbored this variant along with p.Gly863Ala and p.Trp1408Arg. In vitro functional analysis showed a synergistic effect of the variants (p.Arg1640Trp and p.Trp1408Arg) in causing a severe reduction in ABCA4 protein expression. ${ }^{32}$ The p.Gly1961Glu variation has been associated with a milder STGD phenotype; this is consistent with our findings that the patient had a normal full-field ERG with no evidence of either cone or rod dysfunction. The fundus appearance in this patient was similar to the phenotype described previously in individuals carrying the variant p.Arg1640Trp. ${ }^{23}$

Another compound heterozygous mutation (p.Phe191Valfs42/p.Gly172Ser) was found in the patient (SG-02_Il:1) with typical signs of atrophic macula and paramacular flecks in both eyes. The missense amino acid substitution p.Gly172Ser was previously identified in STGD probands from South African and Italian cohorts. ${ }^{33,34}$ The novel essential splice site mutation c.570+1G >A (p.Phe191Valfs42) is predicted to cause mis-splicing of the gene, resulting in a frameshift and subsequent truncation in the protein. A neighboring mutation c.570G $>C$ (p.Gln $190 \mathrm{His}$ ) has previously been reported in compound heterozygous state along with p.Gly1961Glu in a patient with STGD. ${ }^{14}$

We found a homozygous missense mutation (p.Thr971Asn) in a patient (SG-03_II:1) with a severe phenotype of STGD (atrophic macula, extensive flecks and rod-cone dysfunction on ERG). We could not check for p.Thr971Asn in the parents, who were possibly asymptomatic carriers. This missense change (p.Thr971Asn) has been previously observed in a STGD patient but not in any of the 96 healthy control individuals investigated in that study. ${ }^{35}$ The mutation alters a residue within the Walker A ATP binding motif (residues 963-970), adjacent to the NBD1 domain, which is located in a highly conserved region. It has been reported that the NBD1 domain is responsible for basal ATPase activity. ${ }^{36}$ Moreover, it has been shown that the variant $\mathrm{p}$.Thr971Asn leads to reduced ATP-binding capacity and abolishes retinal-stimulated ATP hydrolysis. ${ }^{37}$ 
Patient (SG-04_II:1) had a compound heterozygous change (p.Tyr872X, p.Gly1961Glu) and his unaffected mother harbored only one heterozygous variant (p.Gly1961Glu) in the ABCA4 gene. It is well known that p.Gly1961Glu is found in compound heterozygous state along with another pathogenic variant in STGD. ${ }^{17}$ The truncating mutation p.Tyr872X has not been previously reported. It is found in the topological domain of the ABCA4 chain and leads to premature truncation after 872 amino acids, which is likely to be non functional and would be degraded or not be translated. Interestingly, nearby nonsense mutations in ABCA4, for example, p.Ser878X, has been identified in a Chinese patient with STGD. ${ }^{38}$

Another homozygous mutation (p.Arg2149X) was identified in the young girl (SG-05_V:1) from a consanguineous family with a severe phenotype of STGD. Interestingly, a compound heterozygous combination of p.Arg2149X and another variant IVS45+1G >C has been found in patients who displayed an extensive atrophic appearance of the RPE and abnormal responses from rods and cones. ${ }^{39}$ This mutation (p.Arg2149X) leads to a predicted truncated protein in $A B C$ transporter 2 domain in ABCA4 and has been previously described in STGD patients from Japanese and Caucasian origins. ${ }^{40,41}$ There are very few reports describing the variants in CNGB3, PROM1, PRPH2,ELOVL4 and CRB1 were associated with rare cause of Stargardt or Stargardt-like disease phenotypes. ${ }^{8,10-12}$ Our results suggest that ABCA4 pathogenic mutations might be a major cause in Indian patients with STGD, it has been reported in other ethnic groups as well. $31,33,34,40,42,43$ In this study we found the most frequent ABCA4 mutation p.G1961E in two unrelated STGD patients, it has been reported in patients from African American and European ancestry with the frequencies of $2 \%$ and $11 \%$ respectively. ${ }^{44,45}$ Recently, the different stages of an 'optical gap' have been described on the SD-OCT in patients with p.G1961E mutations. ${ }^{46}$ Stage 1 was characterized by mild disruption of the ellipsoid zone over the fovea, while stage 2 showed a progressive loss resulting in an apparent 'optical gap' on the OCT. Stage 3 showing a collapse of this zone associated with RPE atrophy. In our study cohort, the patients SG-01_Il:1 and SG-04_Il:1 who carried a p.G1961E did not demonstrate any optical gap phenotype on the SD-OCT (Figures 2(a) and 2(d)), and exhibited complete loss of photoreceptors and RPE atrophy in the foveal region.

Through our study, we showed the utilization of next generation sequencing in the precise molecular diagnosis of STGD and helping in clinical situation. However, a single candidate gene could not be selected for genetic analysis, since STGD is associated with variable clinical features can be caused by six other known genes. The 
gold standard for identifying nucleotide changes is direct sequencing, but screening of genes like ABCA4 (large and consists of 50 exons) or other STGD related a gene is expensive and time-consuming. The panel of targeted gene sequencing by NGS might be a cost efficient tool, which enables the rapid sequencing of a large gene like ABCA4 and multiple genes simultaneously in large cohorts of patients with STGD. This could be a conventional method for rapid determination of genotype-phenotype in STGD or Stargardt-like diseases and this method can routinely implemented in future gene therapy approaches in retinal dystrophies. Though we have suggested that performing clinical exome would give us an advantage of validating the clinical diagnosis, in our study cohort we identified nonambiguous STGD phenotype and had ABCA4 gene mutation out of half a dozen other genes. However, this may not be the case when others use the same methodology in their clinics, where there could be clinical and genetic heterogeneity and in such circumstances clinical exome could come in handy for the clinician ${ }^{47}$ in further management of the cases for focused genetic counseling and also in helping parents to make appropriate reproductive choices.

In summary, we report here for the first time, two novel ABCA4 mutations from two unrelated STGD patients of Indian origin. Our genetic study on STGD patients has allowed better understanding of the significance of the mutational landscape in the ABCA4 gene and may help in the genetic counseling of patients and asymptomatic carriers. The identified genetic variations and associated clinical phenotypes were consistent with other studies. We further confirm that screening of ABCA4 mutations in patients with STGD is essential for clinical management and future therapeutic approaches.

\section{References}

1. Weleber RG "Stargardt's macular dystrophy," Arch Ophthalmol 1994; 112; 752-54.

2. Walia S and Fishman GA, "Natural history of phenotypic changes in Stargardt macular dystrophy," Ophthalmic Genet 2009; 30:63-68.

3. Franceschetti $A$ and Francois J, "[Fundus flavimaculatus]," Arch Ophtalmol Rev Gen Ophtalmol 1965; 25:505-530.

4. Birnbach CD, Jarvelainen M, Possin DE and AMilam AH, "Histopathology and immunocytochemistry of the neurosensory retina in fundus flavimaculatus," Ophthalmology 1994;101:1211-1219.

5. Molday RS, Zhong M and Quazi F "The role of the photoreceptor ABC transporter $A B C A 4$ in lipid transport and Stargardt macular degeneration," Biochim Biophys Acta 2009; 1791:573-583. 
6. Molday RS and Zhang K, "Defective lipid transport and biosynthesis in recessive and dominant Stargardt macular degeneration," Prog Lipid Res 2010;49: 476-492.

7. September AV, Vorster AA, Ramesar RS and Greenberg LJ, "Mutation spectrum and founder chromosomes for the ABCA4 gene in South African patients with Stargardt disease," Invest Ophthalmol Vis Sci 2004; 45:1705-1711.

8. Zhang K, Kniazeva M, Han M et al. "A 5-bp deletion in ELOVL4 is associated with two related forms of autosomal dominant macular dystrophy," Nat Genet 2001; 27: 89-93.

9. Coco RM, Telleria JJ, Sanabria MR, Rodriguez-Rua E and Garcia MT, "PRPH2 (Peripherin/RDS) mutations associated with different macular dystrophies in a Spanish population: a new mutation," Eur J Ophthalmol 2010; 20: 724-732.

10. Yang $Z$, Chen Y, Lillo $C$, et al. "Mutant prominin 1 found in patients with macular degeneration disrupts photoreceptor disk morphogenesis in mice," J Clin Invest 2008:118: 2908-16.

11. Nishiguchi KM, Sandberg MA, Gorji N, Berson EL and Dryja TP, "Cone CGMP-gated channel mutations and clinical findings in patients with achromatopsia, macular degeneration, and other hereditary cone diseases," Hum Mutat 2005;25: 248-258.

12. Strom SP, Gao YQ, Martinez A, et al. "Molecular diagnosis of putative Stargardt Disease probands by exome sequencing," BMC Med Genet 2012; 13; 67.

13. Allikmets $R$, Singh $N$, Sun $H$, et al. "A photoreceptor cell-specific ATP-binding transporter gene $(A B C R)$ is mutated in recessive Stargardt macular dystrophy," Nat Genet 1997; 15; 236-246.

14. Briggs $C E$, Rucinski D, Rosenfeld PJ, Hirose T, Berson EL and Dryja TP, "Mutations in $A B C R(A B C A 4)$ in patients with Stargardt macular degeneration or cone-rod degeneration," Invest Ophthalmol Vis Sci 2001;42: 2229-2236.

15. Anderson $K L$, Baird $L$, Lewis RA, et al. "A YAC contig encompassing the recessive Stargardt disease gene (STGD) on chromosome 1p," Am J Hum Genet 1995; 57: 1351-1363.

16. Sun H, Molday RS and Nathans J, "Retinal stimulates ATP hydrolysis by purified and reconstituted $A B C R$, the photoreceptor-specific ATP-binding cassette transporter responsible for Stargardt disease," J Biol Chem 1999; 274: 8269-8281.

17. Cideciyan AV, Swider M, Aleman TS, et al. "ABCA4 disease progression and a proposed strategy for gene therapy," Hum Mol Genet 2009; 18: 931-941.

18. Weng J, Mata NL, Azarian SM, Tzekov RT, Birch DG and Travis GH, "Insights into the function of Rim protein in photoreceptors and etiology of Stargardt's disease from the phenotype in abcr knockout mice," Cell 1999; 98:13-23.

19. Mata NL, Tzekov RT, Liu X, Weng J, Birch DG and Travis GH, "Delayed dark-adaptation and lipofuscin accumulation in abcr+/-mice: implications for involvement of $A B C R$ in age-related macular degeneration," Invest Ophthalmol Vis Sci 2001; 42: 1685-1690. 
20. Sparrow JR and Boulton M, "RPE lipofuscin and its role in retinal pathobiology," Exp Eye Res 2005; 80: 595-606.

21. Maeda A, Maeda T, Golczak M and Palczewski K, "Retinopathy in mice induced by disrupted all-trans-retinal clearance," J Biol Chem 2008; 283: 26684-26693.

22. Wu L, Nagasaki T and Sparrow JR. "Photoreceptor cell degeneration in Abcr (-/-) mice," Adv Exp Med Biol 2010; 664: 533-539.

23. Klevering BJ, Deutman AF, Maugeri A, Cremers FP and Hoyng CB, "The spectrum of retinal phenotypes caused by mutations in the ABCA4 gene," Graefes Arch Clin Exp Ophthalmol 2005; 243:90-100.

24. Cremers FP, van de Pol DJ, van Driel $M$, et al. "Autosomal recessive retinitis pigmentosa and cone-rod dystrophy caused by splice site mutations in the Stargardt's disease gene ABCR," Hum Mol Genet 1998; 7: 355-362.

25. Martinez-Mir A, Paloma E, Allikmets $R$, et al. "Retinitis pigmentosa caused by a homozygous mutation in the Stargardt disease gene ABCR," Nat Genet 1998;18: 11-12.

26. Allikmets $R$, Shroyer NF, Singh N, et al. "Mutation of the Stargardt disease gene (ABCR) in age-related macular degeneration," Science 1997; 277: 1805-1807.

27. Miller SA, Dykes DD and Polesky HF, "A simple salting out procedure for extracting DNA from human nucleated cells," Nucleic Acids Res 1988; 16:1215.

28. Xi Q, Li L, Traboulsi El and Wang QK, "Novel ABCA4 compound heterozygous mutations cause severe progressive autosomal recessive cone-rod dystrophy presenting as Stargardt disease," Mol Vis 2009; 15: 638-645.

29. Hamel CP, "Cone rod dystrophies," Orphanet J Rare Dis 2007; 2: 7.

30. Simonelli F, Testa F, de Crecchio G, et al. "New ABCR mutations and clinical phenotype in Italian patients with Stargardt disease," Invest Ophthalmol Vis Sci 2000; 41: 892-897.

31. Riveiro-Alvarez R, Aguirre-Lamban J, Lopez-Martinez MA, et al. "Frequency ABCA4 mutations in 278 Spanish controls: an insight into the prevalence of autosomal recessive Stargardt disease," Br J Ophthalmol 2009; 93: 1359-1364.

32. Shroyer NF, Lewis RA, Yatsenko AN, Wensel AG and Lupski JR, "Cosegregation and functional analysis of mutant $A B C R$ (ABCA4) alleles in families that manifest both Stargardt disease and age-related macular degeneration," Hum Mol Genet 2001;10: 2671-2678.

33. Roberts LJ, C. A. Nossek CA, Greenberg LJ and Ramesar RS, "Stargardt macular dystrophy: common ABCA4 mutations in South Africa--establishment of a rapid genetic test and relating risk to patients," Mol Vis 2012;18: 280-289.

34. Passerini I, Sodi A, Giambene B, Mariottini A, Menchini U and Torricelli F, "Novel mutations in of the $A B C R$ gene in Italian patients with Stargardt disease," Eye (Lond) 2010; 24: 158-164. 
35. Webster AR, Heon E, Lotery AJ, et al. "An analysis of allelic variation in the ABCA4 gene," Invest Ophthalmol Vis Sci 2001; 42: 1179-1189.

36. Tsybovsky Y, Orban T, Molday RS, Taylor D and Palczewski K, "Molecular organization and ATP-induced conformational changes of $A B C A 4$, the photoreceptor-specific $A B C$ transporter," Structure, vol. 21, no. 5, pp. 854-860, 2013.

37. Sun Hand Nathans J, "Mechanistic studies of $A B C R$, the $A B C$ transporter in photoreceptor outer segments responsible for autosomal recessive Stargardt disease," J Bioenerg Biomembr 2001; 33: 523-530.

38. Yi J, Li S, Jia X, et al. "Evaluation of the ELOVL4, PRPH2 and ABCA4 genes in patients with Stargardt macular degeneration," Mol Med Rep 2012; 6: 1045-1049.

39. Testa F, Rossi S, Sodi A, et al. "Correlation between photoreceptor layer integrity and visual function in patients with Stargardt disease: implications for gene therapy," Invest Ophthalmol Vis Sci 2012; 53: 4409-4415.

40. Fukui T, Yamamoto S, Nakano K, et al. "ABCA4 gene mutations in Japanese patients with Stargardt disease and retinitis pigmentosa," Invest Ophthalmol Vis Sci 2002; 43: 2819-2824.

41. Lewis RA, Shroyer NF, Singh N, et al. "Genotype/Phenotype analysis of a photoreceptor-specific ATP-binding cassette transporter gene, ABCR, in Stargardt disease," Am J Hum Genet 1999; 64: 422-434.

42. Rivera $A$, White $K$, Stohr $H$, et al. "A comprehensive survey of sequence variation in the ABCA4 (ABCR) gene in Stargardt disease and age-related macular degeneration," Am J Hum Genet 2000; 67: 800-813.

43. Rozet JM, Gerber S, Souied E, et al. "Spectrum of ABCR gene mutations in autosomal recessive macular dystrophies," Eur J Hum Genet 1998; 6: 291-295.

44. Burke TR, Fishman GA, Zernant J, et al. "Retinal phenotypes in patients homozygous for the G1961E mutation in the ABCA4 gene," Invest Ophthalmol Vis Sci 2012; 53 : 4458-4467.

45. Zernant J, Collison FT, Lee W, et al. "Genetic and clinical analysis of ABCA4-associated disease in African American patients," Hum Mutat 2014; 35: 1187-1194.

46. Noupuu K, Lee W, Zernant J, Tsang SH and Allikmets R, "Structural and genetic assessment of the ABCA4-associated optical gap phenotype," Invest Ophthalmol Vis Sci 2014; 55: 7217-7226.

47. Blue Cross and Blue Shield Association, "Special report: exome sequencing for clinical diagnosis of patients with suspected genetic disorders", Technology Evaluation Center Assessment Progmran. Executive Summary, vol.27, no.3, pp.1-4, 2013 


\section{CHAPTER 8}

\section{EXOME SEQUENCING IS AN EFFICIENT TOOL FOR VARIANT \\ LATE-INFANTILE NEURONAL CEROID \\ LIPOFUSCINOSIS MOLECULAR DIAGNOSIS}

Rajani Battu, Liliana Catherine Patiño,

Oscar Ortega-Recalde, Jeyabalan Nallathambi,

Venkata Ramana Anandula, Umashankar, Paul Laissue

PLOS ONE. 2014;15; 9(10) 



\section{Abstract}

The neuronal ceroid-lipofuscinoses $(\mathrm{NCL})$ is a group of neurodegenerative disorders characterized by epilepsy, visual failure, progressive mental and motor deterioration, myoclonus, dementia and reduced life expectancy. Classically, NCL-affected individuals have been classified into six categories, which have been mainly defined regarding the clinical onset of symptoms. However, some patients cannot be easily included in a specific group because of significant variation in the age of onset and disease progression. Molecular genetics has emerged in recent years as a useful tool for enhancing NCL subtype classification. Fourteen NCL genetic forms (CLN1 to CLN14) have been described to date. The variant late-infantile form of the disease has been linked to CLN5, CLN6, CLN7 (MFSD8) and CLN8 mutations. Despite advances in the diagnosis of neurodegenerative disorders mutations in these genes may cause similar phenotypes, which rends difficult accurate candidate gene selection for direct sequencing. Three siblings who were affected by variant late-infantile $\mathrm{NCL}$ are reported in the present study. We used whole-exome sequencing, direct sequencing and in silico approaches to identify the molecular basis of the disease. We identified the novel c.1219T.C (p.Trp407Arg) and c.1361T.C (p.Met454Thr) MFSD8 pathogenic mutations. Our results highlighted next generation sequencing as a novel and powerful methodological approach for the rapid determination of the molecular diagnosis of NCL. They also provide information regarding the phenotypic and molecular spectrum of CLN7 disease. 


\section{Introduction}

The neuronal ceroid-lipofuscinoses (NCL), also known as Batten Disease, is a group of neurodegenerative disorders which is characterised by the accumulation of autofluorescent storage positive material in the cytoplasm of neurons. ${ }^{1,2}$ This feature can also be found in other tissues, such as the skin and skeletal muscle. ${ }^{3} \mathrm{NCL}$ is one of the most frequently inherited childhood onset neurodegenerative pathologies since its prevalence ranges from 1:100.000 to 1:1.000.000. ${ }^{2}$ From a clinical point of view, NCL patients suffer from epilepsy, visual failure, progressive mental and motor deterioration, myoclonus, dementia and reduced life expectancy. ${ }^{1}$ Classically, NCLaffected individuals have been classified into six categories (congenital, infantile, late infantile, variant late infantile, juvenile and adult) which have been mainly defined regarding the clinical onset of symptoms. ${ }^{4}$ However, some patients cannot be easily included in a specific group because of significant variation in the age of onset and disease progression. Molecular genetics has emerged in recent years as a useful tool for enhancing NCL subtype classification. Fourteen NCL genetic forms (CLN1 to CLN14) have been described to date. ${ }^{2,5}$ More than $360 \mathrm{NCL}$ aetiological mutations have been reported, most of which have been included in the NCL Mutation Database (http:// www.ucl.ac.uk/ncl/mutation). ${ }^{6}$

To date, the variant late infantile form of the disease has been linked to CLN5, CLN6, CLN7 and CLN8 mutations. ${ }^{2,7}$ The MFSD8 (CLN7) gene, which is located on chromosome 4q28.1-q28.2, encodes for the MFSD8 (major facilitator superfamily domain-containing protein 8 ) protein. ${ }^{8}$ This factor belongs to the major facilitator superfamily (MFS) family of membrane transport proteins which are involved in transporting small solutes through cell membranes. It has been suggested that MFSD8 acts as a lysosomal transporter in eukaryotic cells. Although this protein is ubiquitously expressed, high transcript concentrations have been identified in specific brain locations, such as the cerebellar cortex and the hippocampus. ${ }^{9}$

More than 30 pathogenic sequence variants have been described so far inMFSD8, most being homozygous missense mutations (www.ucl.ac.uk/ncl/mutation). ${ }^{2}$ It has been proposed that this kind of mutations does not affect protein subcellular location but might produce disturbances regarding their functional properties ${ }^{6}$ (and references therein). Almost all MFSD8 mutations are isolated and some founder effects have been described. ${ }^{10}$ Despite advances in the diagnosis of neurodegenerative disorders, CLN7 
disease remains clinically difficult to distinguish from other forms of late-infantile lipofuscinosis. Molecular testing thus constitutes an indispensable technique for establishing an accurate diagnosis and facilitating genetic counselling.

Three siblings who were affected by variant late-infantileNCL are reported in the present study. Whole-exome sequencing led to identify the novel c.1219T.C (p.Trp407Arg) and c.1361T.C (p.Met454Thr)MFSD8 pathogenic mutations and to define the clinical and molecular diagnosis precisely. These results highlighted next generation sequencing as a powerful methodological approach for the rapid determination of the molecular basis of NCL. They also provide information regarding the phenotypic and molecular spectrum of CLN7 disease.

\section{Material and Methods}

\section{Patients}

The patients (P1-IV:1, P2-IV:2 and P3-IV:4) and a non-affected son (C3-IV:3) were siblings who were attending Retina clinic at Narayana Nethralaya, (Bangalore, Karnataka, India), along with their parents (C1-III:6 and C2-III:7) (Figure 1A). This study was approved by the Universidad del Rosario's and Narayana Nethralaya eye hospital's Ethics Committees and was conducted in line with the Declaration of Helsinki. The parents gave their written informed consent and signed on behalf of their children. The patients' parents were reported to be consanguineous. All three patients (P1, P2 and P3) failed a school vision test at the age of 5-6 years and were referred for an eye check-up. All three children subsequently developed seizures and regression of motor milestones noted in the older two siblings.

P1 is a 14-year-old male child seen at the age of 5 years with rapid decrease in vision in both eyes. Vision at presentation was $6 / 90$ (Snellen's) in both eyes. Fundus examination showed severe optic disc pallor and arteriolar attenuation. A full field electroretinogram (ERG) showed severe rod-cone dysfunction. He developed seizures at the age of 7 years, and was noted to have progressive neurological illness in the form of recurrent myoclonic jerks and generalized tonic clonic seizures. There was a progressive cognitive decline and visual impairment. Last examination showed vision of perception of light ( $\mathrm{PL}$ ) in both eyes. Currently, he is completely bedbound with very poor vision and uncontrolled seizures on multiple anticonvulsants. Electroneuromyogram done on the upper limbs showed bilateral CPN axonal neuropathy. MRI brain was not available for this patient. 
FIGURE 1.

A) Pedigree of the NCL family. Black symbols refer to affected individuals. Half-black symbols into third and fourth generation individuals symbols (III:6, III:7, IV:3) represents the MFSD8 c.1219T.C and c.1361T.C mutations at heterozygous state.B) Brain magnetic resonance imaging (T2-FLAIR image, axial section) of patient (P3) showing mild cerebral atrophy.doi:10.1371/journal.pone.0109576.g001

A.

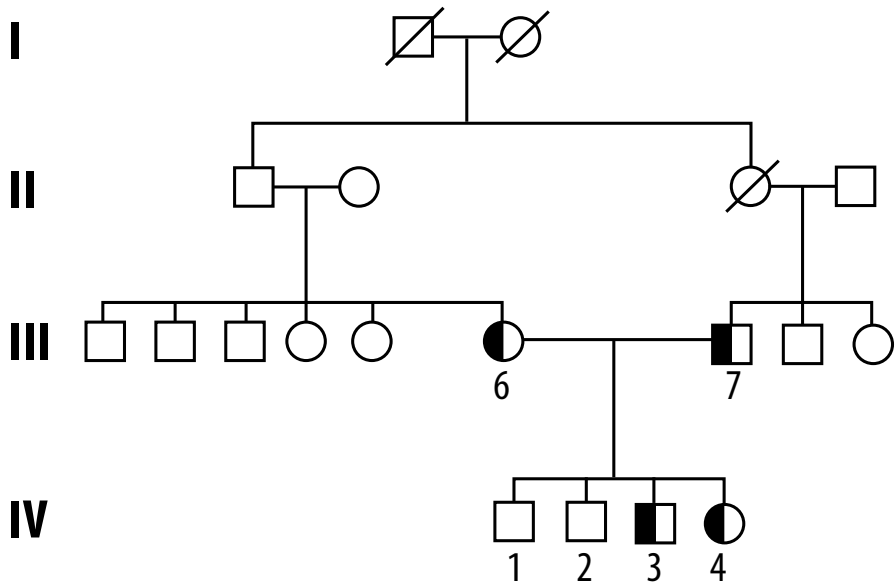

B.

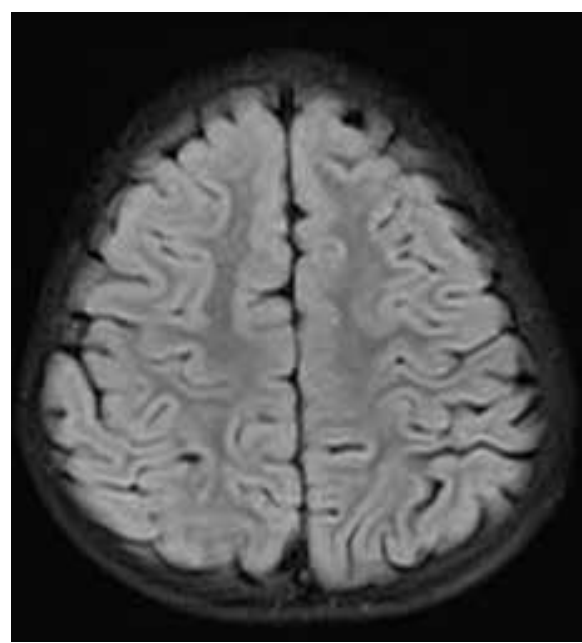

P2 is a 12-year-old male who was referred since he failed a school vision examination at the age of 6 years. He had useful vision at birth. Examination showed a visual acuity of $2 / 60$ in both eyes. Fundus showed bilateral pale optic discs, severe arteriolar attenuation with diffuse pigment mottling throughout the fundus and few bony spicules. Spectraldomain optical coherence tomography (SD-OCT) showed diffuse loss of photoreceptors. Full field ERG showed severe attenuation of the rod and cone responses. He developed seizures at the age of 7 years with a regression of motor milestones. When examined last (at the age of 12 years), his vision was PL in both eyes. He had slurred speech with difficulty in walking and needed assistance. Neurological examination showed ataxia with brisk reflexes in both upper and lower limbs. MRI brain showed diffuse cerebral and cerebellar atrophy with normal thalamus (Figure 1B). Electroencephalogram revealed a normal background and generalised spikes, polyspike and wave activity.

P3 is an 8-year-old female child who first presented at the age of 5 years with visual failure in both eyes. Examination showed visual acuity of counting fingers close to face in the right eye (RE) and 6/90 on Snellen's chart in the left eye (LE). Fundus showed 
bilateral optic disc pallor with severe arteriolar attenuation and diffuse pigment mottling. SD-OCT showed severe loss of photoreceptors and foveal thinning. A full field ERG showed severe rod-cone dysfunction. The child developed seizures at the age of 6 years. MRI brain showed mild cerebral atrophy, normal cerebellum and thalamus. On last examination, vision was PL in both eyes, her seizures were under control with medications. The unaffected sibling (C3) and his parents ( $\mathrm{C} 1$ and $\mathrm{C} 2$ ) had normal vision, normal fundus examination and no seizures.

\section{Exome Capture and High-throughput Sequencing}

DNA from the parents and three of the children (P2, P3 and C3) was extracted from blood lymphocytes, using standard procedures. DNA samples could not be obtained from $\mathrm{P} 1$. The P2, P3 and C3 samples were subjected to whole-exome sequencing. This involved 3.5 micrograms of each sample being made up to $100 \mathrm{ul}$ with TE and sonicated to fragment DNA into sizes ranging from $100 \mathrm{bp}$ to $200 \mathrm{bp}$ (Covaris). Such fragmented DNA was cleaned up using Agencourt AMPure XP beads (Beckman Coulter). Size distribution was checked by running an aliquot of the sample on an Agilent High Sensitivity Bioanalyzer chip. Genomic DNA libraries were then constructed using Ion TargetSeq Exome Enrichment for the Ion Proton System (Part \# MAN0006730, revision 5.0). End-repair and adapter ligation were done according to established protocol (Ion plus fragment library kit \# 4471252). The samples were cleaned using Ampure XP beads. The samples were size-selected on gel at $200 \mathrm{bp}$ and eluted using MinElute columns. Adaptor-ligated fragments were enriched by PCR amplification. The prepared libraries were pooled in equal amounts ( $167 \mathrm{ng}$ each) and concentrated using a vacuum concentrator to give $\sim 500 \mathrm{ng}$. The library fragments were captured in solution using $\sim 2$ million TargetSeq capture probes, producing biotinylated oligos ranging in size from $\sim 50$ bases to $\sim 120$ bases (470C for 66 hours). Hybridisation specificity was ensured by using blocker DNA sequences (Human Cot-1 DNAR Fluor QC and Ion TargetSeq Blockers). Bound DNA was isolated using M 270 streptavidincoated DynabeadsR paramagnetic beads and then amplified and purified. An aliquot of the captured library was run on an Agilent High Sensitivity Bioanalyzer Chip. Real time PCR validation involved using pre- and post-capture libraries for observing capture efficiency. The purified, exome-enriched library was then used to prepare a template on particles for preparing enriched, template-positive Ion $\mathrm{PI}$ ion sphere particles (ISPs) for sequencing on an lon PI chip to obtain the necessary data coverage. 


\section{Reference Genome and RefSeq Database}

The human reference genome GRCh37/hg19 was used for mapping exome-sequencing (Exome-Seq). The sequence database downloaded from the lon Torrent reference website (http://updates.iontorrent.com/reference/hg19.zip) was used as our gene model and for determining amino acid substitutions.

\section{Exome-Seq Processing - Read Mapping, Variant Calling and Effect Determination}

Raw data (which is available upon request) was obtained from the lon Proton sequencer and lon Suite was used for checking data quality and filtering. The high quality filtered data was aligned with the human reference genome and variants were named using lon Reporter v1.6 (Ion Suite). Variations (vcffile) were then transferred to a local Linux machine. The filtered variations were annotated, based on their function (e.g. synonymous, nonsynonymous) and their potential effect by using Ingenuity software plugin within the lon Reporter. The results were further compared with the dbSNP database NCBI version $\mathrm{db}$ 137. This result helped to identify novel variations and identify their effect. Finally, the annotated variations were further reviewed manually. Genotypic Technology (Bangalore, Karnataka, India) did the exome sequencing and primary data analysis. The complete filtered data has been included as Tables S1, S2 and S3.

\section{MFSD8 Direct Sequencing and in Silico Analysis}

The MFSD8 exon 12 encoding sequence (ENST00000296468) was amplified by PCR in all available DNA samples from the family members using exon-flanking oligonucleotides. Amplicons were purified using shrimp alkaline phosphatase and exonuclease $\mathrm{I}$, and sequenced with internal primers using Sanger sequencing method (primer sequences and PCR conditions are available on request). ClustalW software was used for aligning the human MFSD8 wild type sequence (Uniprot ID: Q8NHS3) with those from the following vertebrate species:Pan troglodytes (H2QQ56), Mus musculus (Q8BH31), Bos taurus (E1BPY5), Xenopus laevis (Q6GPQ3) andDanio rerio (Q0VA82). SIFT (http://sift.bii.a-star. edu.sg/) and PolyPhen2 (http://genetics.bwh.harvard.edu/pph2/) software was used for ascertaining potential deleterious effects caused by the p.Trp407Arg and p.Met454Thr mutations. Briefly, PolyPhen2 and SIFT prediction values resulted from algorithms which included protein characteristics such as comparative analysis of sequences from different species and exchanged amino acids' physicochemical characteristics. SIFT values lower than 0.06 were considered potentially pathogenic. PolyPhen 2 results were 
assessed as being probably damaging (more confident prediction), possibly damaging (less confident prediction) or benign (non-pathogenic). The Amino Acid Mutation Stability Prediction Server MUpro (version 1.0, http://mupro.proteomics.ics.uci.edu/) was used to study potential MFSD8 stability mpairment due to the p.Trp407Arg and p.Met454Thr mutations. ${ }^{11}$

\section{Results}

\section{Whole-Exome Sequencing}

Whole-exome sequencing produced $\sim 3.4 \mathrm{~GB}$ data for 3 samples for each individual as single-end, having $113 \mathrm{bp}$ mean read length and about 76\% (50 Mb in length) of the targeted bases were covered at $\sim 44$ average read depth which sufficiently passed our thresholds for calling SNPs and short insertions or deletions (indels). Bases having quality scores above 20 (99\% accuracy regarding a base call) represented over $80 \%-82 \%$ of total sequence data. NGS data filtering revealed that P2 and P4 shared 2 homozygous missense variants located on theMFSD8 coding region:c.1219T.C(p. Trp407Arg)andc.1361T.C (p.Met454Thr). NGS showed that C3 was a heterozygous carrier for the p.Trp407Arg and p.Met454Thr mutations. These variants were not present in SNP public databases (e.g. dbSNP database NCBI version db 137).

Direct Sequencing and in Silico Analysis of the MFSD8 p.Trp407Arg and p.Met454Thr Mutations

Direct sequencing confirmed the aforementioned NGS results and revealed that P2 and P4 had inherited mutant alleles from their parents. Multiple protein alignment revealed a strict conservation, during vertebrate species' evolution, of both tryptophan and methionine residues at 407 and 454 positions. SIFT was 0.00 and Polyphen2 1.00 ("probably damaging") for both mutations. Analysis of amino acid mutation stability for p.Trp407Arg and p.Met454Thr mutations identified in this study using MUpro software suggested a decrease in the stability of MFSD8 protein structure. 
FIGURE 2.

Chromatograms showingMFSD8c.1219T.C (A) and c.1361T.C (B) mutations.

WT: wild type sequence. C1: control 1 (III:6).C2:control 2 (III:7). P2: patient 2 (IV:2). C3: control 3 (IV:3). P3: patient (IV:4). Asterisks show the accurate position of relevant mutations.

A.

c.1219T>C

(p.Trp407Arg)

WT AACA AGCC I GGTGCCTC

C1

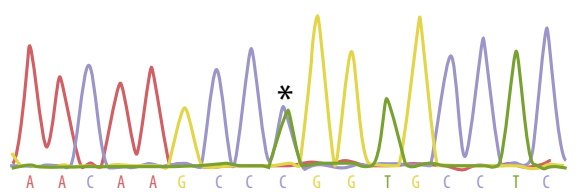

C

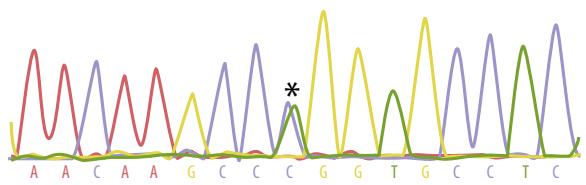

P2

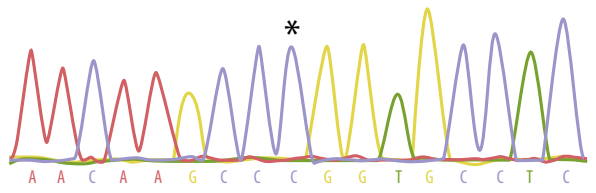

C3

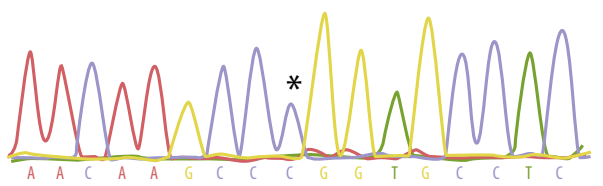

P3

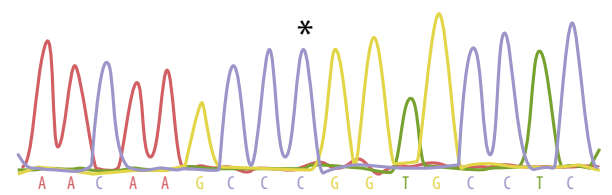

B.

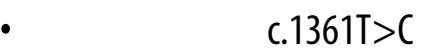

(p.Met454Thr)

WT TGTATACA I GGGCTGGT

C1

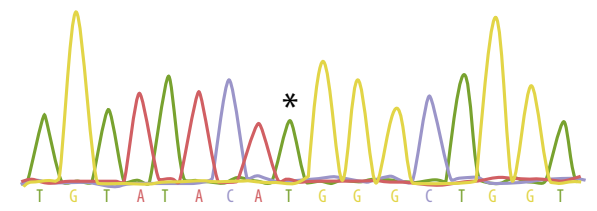

C2

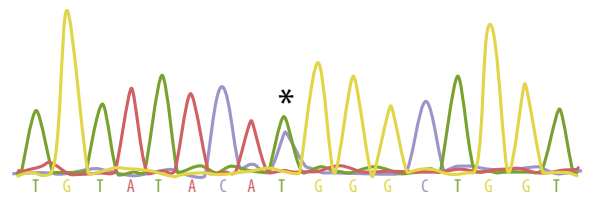

P2

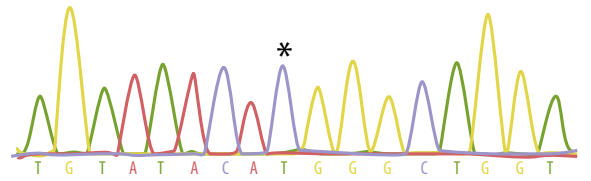

C3

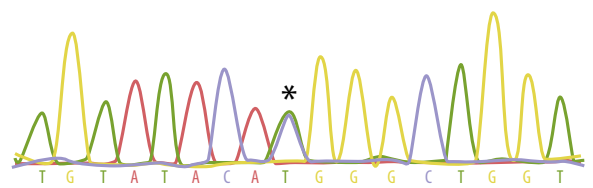

P3

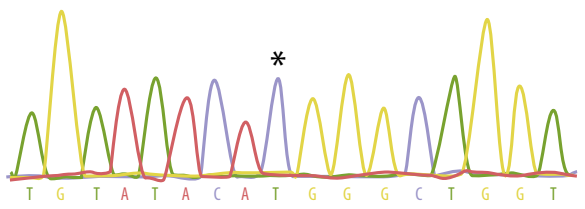

doi:10.1371/journal.pone.0109576.9002 
FIGURE 3.

Comparative sequence analysis of MFSD8 orthologues. Residues at position 407 (W) and 454 (M) are indicated in bold and highlighted with grey shading. doi:10.1371/journal.pone.0109576.g003

\begin{tabular}{|c|c|}
\hline H.sapiens & $\begin{array}{cc}407 & 454 \\
\text { CSIEQA } \frac{1}{W} \text { CLYTPVIHLAQFLTSAVLIGLGYPVCNLMSYTLYSKILGPKPQGVYMัGWLTA }\end{array}$ \\
\hline P.troglodytes & CSIEQAWCLYTPVIHLAQFLTSVVLIGLGYPVCNLMSYTLYSKILGPKPQGVYMGWLTA \\
\hline M.musculus & CSIEQAWCLYTPVIHLAQFLTAAVLIGLGYPACSVMSYTLYSKVLGPKPQGIYMGWLTT \\
\hline B. taurus & AWCLYTPMIHLAQFLISAVLIGIGYI \\
\hline X. laevis & CPVIQTWCLYTPVIHLAQYLTSDILIGVGYPICNVMSYTLYSKIIGPKPQGLYMGWLTA \\
\hline D.rerio & CPSEQTWCLFTPVIHLAQYITSDILIGVGYPTCNVMSYTLYSKILGPKPQGVYMGWLTA \\
\hline
\end{tabular}

\section{Discussion}

A rapid clinical diagnosis of $\mathrm{NCL}$ may be particularly challenging since clinical signs may be unspecific, similar to many other neurodegenerative disorders. Electron microscopy, showing ultrastructural lipofuscinic pigments, has been used for diagnosing NCL.CLN genotyping has led to establishing molecular diagnosis, which may be related to specific clinical subtypes. However, mutations in distinctCLN genes may cause similar phenotypes, thereby hampering candidate gene selection for direct sequencing. The patients in the present study were affected by a recessive neurodegenerative disease, mainly characterised by progressive visual dysfunction, epilepsy and gradual regression of milestones.

Such features, as well as a family history of consanguinity, were clinically evocative of a recessive late infantile onset form of ceroid lipofuscinosis. However, a single candidate gene could not be selected for direct sequencing, since CLN2, CLN5, CLN6, CLN7 and CLN8 patients may share clinical features to those presented by our patients. This would have meant that definitive molecular diagnosis by direct sequencing would have been time-consuming and expensive. Our group, and others, have previously shown that whole-exome sequencing is a cost-effective approach to establishing a molecular diagnosis of Mendelian recessive diseases, particularly when mutations in distinct genes are related to overlapping phenotypes. ${ }^{12,13}$ Therefore, for rapid determination of the molecular aetiology we performed whole-exome sequencing for two of the affected children (P2 and P4) and their unaffected brother (C3) (Figure 1). Since the family tree revealed consanguinity, it was estimated that the most likely genetic hypothesis was related to a homozygous non-synonymous pathogenic mutation in the patients. NGS data analysis revealed that P2 and P3 shared a total 
of 2 novel MFSD8 homozygous coding variants (c.1219T.C, p.Trp407Arg and c.1361T.C, p.Met454Thr) (Figure 2). Direct sequencing confirmed that the patients' relatives (C1, C2 and (3) carried both variants at heterozygous state.In silico analysis of the p.Trp407Arg and p.Met454Thr mutation revealed that the $W$ and $M$ residues (in 407 and 454 positions) had been strictly conserved during vertebrate species evolution, thereby underlining its critical functional role (Figure 3). Furthermore, these mutations implied drastic modification in terms of physicochemical properties. For instance, the $\mathrm{W}$ is a hydrophobic aromatic amino acid while $\mathrm{R}$ is a positively-charged residue. Accordingly, SIFT and Polyphen bioinformatics tools predicted a deleterious effect for both substitutions as they determined complete intolerance for the arginine and threonine residue at positions 407 and 454, respectively. Furthermore the MuPro bioinformatics tool predicted MFSD8 protein instability due to the p.Trp407Arg and p.Met454Thr mutations. These findings, as well as the lack of theses variants in public SNP databases, led to functionally linking them to the patients' phenotype. The p.Trp407Arg mutation was located in a highly conserved region of amino acids between the MFSD8 protein's $9^{\text {th }}$ and $10^{\text {th }}$ transmembrane domains, while the p.Met454Thr mutation is located on the $11^{\text {th }}$ transmembrane domain. This might have been related to correct polypeptide folding and protein's normal function. It is important to note that these two novel variations were located in the same disease allele because the unaffected patients' parents and C3 were carriers of both mutations at heterozygous state.

Taken together, our results demonstrate that whole-exome sequencing is a powerful diagnostic tool for enabling the rapid determination of variant late-infantile neuronal ceroid lipofuscinosis molecular aetiology. We estimate that this method might be commonly used to determine the molecular basis of NCL. We also provide clinical information regarding this type of patient, which might be useful for future genotypephenotype correlations. We hope that these findings will contribute to genetic counselling especially for consanguineous families with NCL. Finally, functional tests are necessary to establish the accurate pathogenic effect of mutations described in the present study.

\section{References}

1. Haltia M. The neuronal ceroid-lipofuscinoses. J Neuropathol Exp Neurol 2003; 62: 1-13

2. Haltia M, Goebel HH. The neuronal ceroid-lipofuscinoses: a historical introduction. Biochim Biophys Acta 2013; 1832:1795-800 
3. Anderson GW, Goebel HH, Simonati A. Human pathology in NCL. Biochim Biophs Acta 2013; 1832: 1807-26

4. Mole SE, Williams RE, Goebel HH. Correlations between genotype, ultrastructural morphology and clinical phenotype in the neuronal ceroid lipofuscinoses.

Neurogenetics 2005; 6:107-26.

5. Williams RE, Mole SE. New nomenclature and classification scheme for the neuronal ceroid lipofuscinoses. Neurology 2012; 79:183-91.

6. M. Kousi, A.E. Lehesjoki, S.E. Mole. Update of the mutation spectrum and clinical correlations of over 360 mutations in eight genes that underlie the neuronal ceroid lipofuscinoses, Hum. Mutat 2012; 33: 42-63.

7. Jalanko A, Braulke T. Neuronal ceroid lipofuscinoses. Biochim Biophys Acta 2009; 1793:697-709.

8. Siintola E, Topсu M, Aula $N$, et al. The novel neuronal ceroid lipofuscinosis gene MFSD8 encodes a putative lysosomal transporter. Am J Hum Genet 2007; 81:136146.

9. Sharifi A, Kousi M, Sagné C, et al. Expression and lysosomal targeting of CLN7, a major facilitator superfamily transporter associated with variant late-infantile neuronal ceroid lipofuscinosis. Hum Mol Genet 2010; 19:4497-514

10. Kousi M, Siintola E, Dvorakova L, et al. Mutations in CLN7/MFSD8 are a common cause of late-infantile neuronal ceroid lipofuscinosis. Brain 2009. 132: 810-819

11. Cheng J, Randall A, Baldi P. Prediction of protein stability changes for single- site mutations using support vector machines. Proteins 2006; 62:1125-32.

12. Bamshad MJ, Ng SB, Bigham AW, et al. Exome sequencing as a tool for Mendelian disease gene discovery. Nature reviews Genetics 2011; 12: 745-755.

13. Ortega-Recalde $O$, Vergara II, Fonseca DJ, et al. Whole-exome sequencing enables rapid determination of xeroderma pigmentosum molecular etiology. PLoS One 2013; 8:e64692. 



\section{GENETIC ANALYSIS AND CLINICAL PHENOTYPE OF TWO INDIAN FAMILIES WITH X-LINKED CHOROIDEREMIA}

Rajani Battu, Nallathambi Jeyabalan, Praveen Murthy, Kavita S Reddy, Jan SAG Schouten, Caroll AB Webers INDIAN J OPHTHALMOL 2016;64(12):924-29. 



\section{Abstract}

PURPOSE: This study aims to describe the phenotype and genotype of two Indian families affected with X-linked Choroideremia (CHM).

MATERIALS AND METHODS: In these two families, the affected individuals and unaffected family members underwent a comprehensive ophthalmic examination including an optical coherence tomography (OCT) and electroretinogram. Blood samples were collected from the families for genetic analysis. NGS was done using panel of 184 genes, which covered previously associated genes with retinal dystrophies. Sequencing data was analyzed for the CHM, RPGR and RP2 genes that have been implicated in Choroideremia and X-linked RP (XLRP) respectively. The identified variants were confirmed by Sanger sequencing in available individuals and unrelated controls.

RESULTS: In two unrelated male patients, NGS analysis revealed a previously reported 3'- splice site change c.820-1G >C in the CHM gene in the first family and hemizygous mutation c.653G >C (p.Ser218X) in the second family. The asymptomatic family members were carriers for these mutations. Spectral domain-OCT showed loss of outer retina, preservation of the inner retina and choroidal thinning in the affected males and retinal pigment epithelial changes in the asymptomatic carriers. The identified mutations were not present in 100 controls of Indian origin. There were no potential mutations found in XLRP -associated (RPGR and RP2) genes.

CONCLUSIONS: This report describes the genotype and phenotype findings in patients with CHM from India. The identified genetic mutation leads to lack of Rab escort protein-1 (REP-1) or affects the production of a REP-1 protein that is likely to cause retinal abnormalities in patients. 


\section{Introduction}

Choroideremia (CHM) (OMIM 303100) is a rare X-chromosome linked chorioretinal dystrophy characterized by progressive loss of vision due to degeneration of the choroid, retinal pigment epithelium, and photoreceptor cells in retina. ${ }^{1,2}$ Being an X-linked recessive condition, Choroideremia mainly affects males; carrier females are mostly asymptomatic, although those with skewed X-inactivation may become symtomatic later in life. Associated with night blindness and reduced peripheral fields, the disease progresses relentlessly till most affected males are legally blind by midlife. Asymptomatic carrier females show irregular pigmentation throughout the fundus. ${ }^{3}$ The global prevalence of the disease is estimated at 1 in 50,000-100,000. ${ }^{4}$ The highest incidence of CHM is reported in northern Finland. ${ }^{5} \mathrm{CHM}$ (\#300390), encoding the protein Rab escort protein-1 (REP-1) is the gene associated with Choroideremia. The causative gene has been mapped at Xq21.1-q21.3, which consists of 15 exons and encodes the 653 aminoacids of intracellular protein known as REP-1. This is involved (Rab GTPases) in the regulation of intracellular vesicle transport and post-translational modification in lipid prenylation (Rab geranylgeranyltransferase), in addition to vesicle trafficking in various vesicular compartments of the cell. ${ }^{6,7}$ The REP-1 protein is actively expressed in ocular and non-ocular tissues such as retina, choroid, RPE and lymphocytes. ${ }^{8}$ Mutations in CHM gene results in Choroideremia phenotype. The spectrum of $\mathrm{CHM}$ mutations include deletions, translocations and point mutations (including nonsense, frame shift and splice site) as well as deep intronic mutations. ${ }^{9-11}$ In mice, the CHM knowck out (KO) is embryonically lethal, but conditional knockout mice of REP-1 showed age related changes in RPE ${ }^{12,13}$ Recombinant adeno-associated virus ( $\mathrm{rAAV}$ ) mediated transduction of human REP-1- restore the enzyme activity REP1 in vitro in cells from affected patients (CHM), suggesting that gene therapy approach by AAV vectors may be an appealing treatment strategy. ${ }^{14,15}$ In the present study, we report for the first time CHM gene mutations and associated clinical features in two Indian patients with $\mathrm{CHM}$

\section{Materials and Methods}

\section{Patient Details and Clinical Eevaluations}

This study was approved by the Institutional Ethical Committee and conducted according to the Declaration of Helsinki Principles. All study participants including 
two patients, two available family members and 108 normal controls provided a written consent to participate in the study. None of the participants had any systemic ilness. There were no hearing or cognitive abnormalities. The proband and family members in two families identified with CHM underwent a complete ophthalmic examination including measurement of visual acuity, intraocular pressure, slit lamp biomicroscopy, detailed fundus examination, fundus photography, fundus autofluorescence (FAF), spectral-domain optical coherence tomography (SD-OCT). Four out of the five participants underwent full field ERG according to ISCEV standards. ${ }^{16}$ (VERIS systems, USA)

\section{Genetic Analysis}

A detailed family history was obtained from all participants. DNA was isolated from $5 \mathrm{ml}$ of peripheral blood by the salt precipitation method. ${ }^{17}$ Targeted next-generation sequencing (NGS) was carried out using a method described previously that included sample preparation, target enrichment and exome sequencing data analysis, variant calling. ${ }^{18}$ For the variant interpretation, CHM (for Choroidermia) and RPGR and RP2 genes (for X-linked RP [XLRP]) were included for the annotation, prioritization, and reporting following the ACMG guidelines. To revalidate the potential CHM variations in patients and in the available family members, 108 unrelated normal controls were analysed using polymerase chain reaction (PCR) primers reported elsewhere. ${ }^{19}$ The PCR products were sequenced (3500 DX, Lifetech Inc., USA) by Sanger sequencing method. The sequence electropherogram was visualized using the Finch TV software (Geospiza, USA) and the presence of the variation in genes was evaluated by comparing the patient's sequence with reference sequence (NM_000390.2).

\section{Results}

The genetic analysis revealed two previously reported known mutations in the CHM gene (p.Ser218X) (c.820-1G>C at intron 6) in two unrelated patients with CHM. There were no potential mutations found in XLRP-associated, RPGR and RP2 genes.

\section{Family 1: Choroideremia-01}

In this family, a 32-year old male patient (IV:7) presented with night blindness and defective vision since childhood (Figure 1). His vision in both eye was 6/6, N6 on Snellen's charting ( $R E=-4.00 \mathrm{DS}, \mathrm{LE}=-3.5 \mathrm{DS})$. The intraocular pressure and anterior segment examinations in both eyes were normal. The lens was clear. The optic discs were pink with a C/D ratio was 0.1 in both eyes. Bilateral fundus findings included the following: prominent areas of atrophy of both the choroid and RPE in the peripapillary 
region, patchy pigment clumping and prominence of large choroidal vessles in the midperiphery, arteriolar attenuation and scalloped margins of choroidal loss with relative sparing of the central retina. FAF of both macula showed a widespread loss of the normal autofluorescence. SD-OCT of both eye showed significant distortion of the outer retinal layers with multiple retinal tubulations (Figure $2 A$ ). There was no cystoid changes in the macula. Full field ERGs showed a complete extinction of both the rods and cone responses. His 61-year old mother was asymptomatic (III:5), she had undergone cataract surgery a year before this, and did not have any significant refractive error before the cataract surgery. Her vision in both eyes was $6 / 6$, N6. Anterior segment examination showed bilateral pseudophakia with a normal intraocular pressure. Fundus examination in both eyes showed widespread RPE alterations with normal retinal and choroidal vessels. FAF both eyes showed patchy hyper and hypoautofluorescence throughout the fundus. SD-OCT of both eyes showed significant undulations in the RPE with preservation of all retinal layers. (Figure $2 B$ ) Full-field ERG of both eyes was normal. The 36-year old sister of the proband was asymptomatic (IV:5), her vision was 6/6, N6 in both eyes. Intraocular pressures and anterior segments and fundus of both eyes were normal. Both eyes showed a normal autofluorescence and normal layers on the SD-OCT. In the proband (IV:7) the NGS analysis revealed a reported 3'-splice site (c.820-1G>C) at intron 6 of CHM gene on the X-chromosome. Patient's mother (III:5) was a heterozygous carrier for the mutation which was absent in the patient's sister (IV:5). This variation leads to altered splice site that results in skipping of exon 7. It codes for amino acids 274 (Val-274) to 314 (Gly314), however, the codon for Gly314 was shared between exon 7 and exon 8, and thus will lead to a frame shift in the open reading frame. Ophthalmic examination of the proband's father (III:6) was normal. Historically, the mother's brother and three of her uncles were legally blind (not examined), likely due to advanced CHM.

\section{Family-2: Choroideremia-02}

A 39-year old male patient (III:3) presented with night blindness since the age of 5 years, other family members had no visual complaints, and there was no history of consanguinity among the parents (Figure 1). Ophthalmic examination showed a visual acuity of 6/9, N6 in both eyes (RE= -4.00 DS/-1.75 DC 800, LE=-4.5 DS/-1.0 DC 1100). Intraocular pressures and anterior segment were normal in both eyes. The lens was clear in both eyes. Fundus evaluation showed normal discs with a C/D ratio of 0.5 . Bilateral fundus findings included the following: areas of prominent loss of choroid and RPE nasal to the disc and arteriolar attenuation with prominent choroidal vessels 
in the midperiphery. Macula showed patchy pigment clumping, scalloped areas of choroidal loss and a very small island of normal retina. FAF showed very small areas of hyperautofluorescence at the macula, corresponding to the remaining visual field. SD-OCT showed advanced loss of photoreceptors with choroidal thinning with retinal tubules in the outer nuclear layer at the junction of the normal and atrophic retina. There were a few cystoid changes at the macula in the $L E$, there were no cystoid changes in the RE. Visual fields showed a small island of remaining visual field on the Humphrey's 10-2. (Figure 3A) Full field ERG showed an extinguished rod response with minimal cone response. Genetic analysis of the patient (III:3) revealed a C to $\mathrm{G}(\mathrm{c} .653 \mathrm{C}>\mathrm{G})$ denovo hemizygous nucleotide change found in the $\mathrm{CHM}$ gene. It was a known nonsense mutation resulting in a premature stop codon (p.Ser218X) found at exon 5 in the canonical transcript (NM_0003 90.2). The truncated protein would lack a major portion of 436 amino acids at the C-terminal region of the REP-1. The patient's 13-year old daughter (IV:1) was asymptomatic, had a heterozygous change (c.653C>G, p.Ser218X)) in the CHM. Her uncorrected vision of 6/6, N6 both eyes, intraocular pressure and anterior segment examination was normal in both eyes. Fundus of both eyes showed normal discs, normal retinal and choroidal blood vessels with areas of retinal pigment alterations throughout the retina. FAF both eyes showed patchy hyperautofluorescence throughout the fundus. SD-OCT of both eyes showed RPE undulations corresponding to the areas of pigmentation (Figure 3B). Full-field ERG in both eyes was normal. Neither the mother nor the sister was available for ophthalmic or genetic evaluation. 
FIGURE 1.

( $a$ and b) Pedigrees of two unrelated families (choroideremia -01, Choroideremia -02) with choroideremia. The asterisk denotes the individuals had an unknown cause of blindness. Arrow indicates the proband in each family. The' + 'symbol denotes the individuals did not consent for the genetic analysis.

A.

CHM - 01
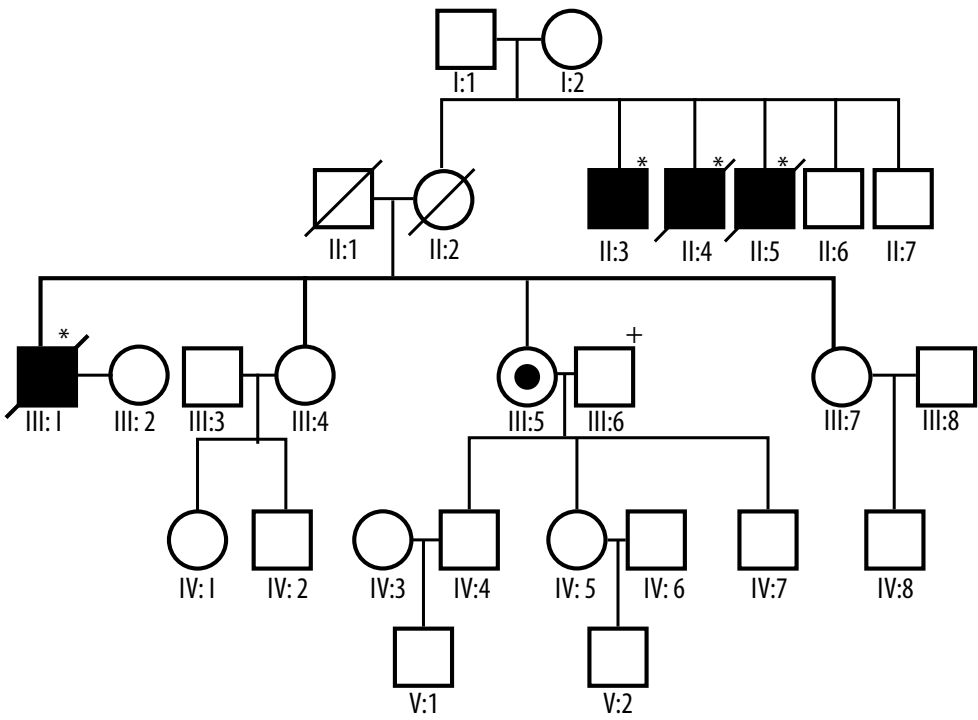

B

CHM - 02

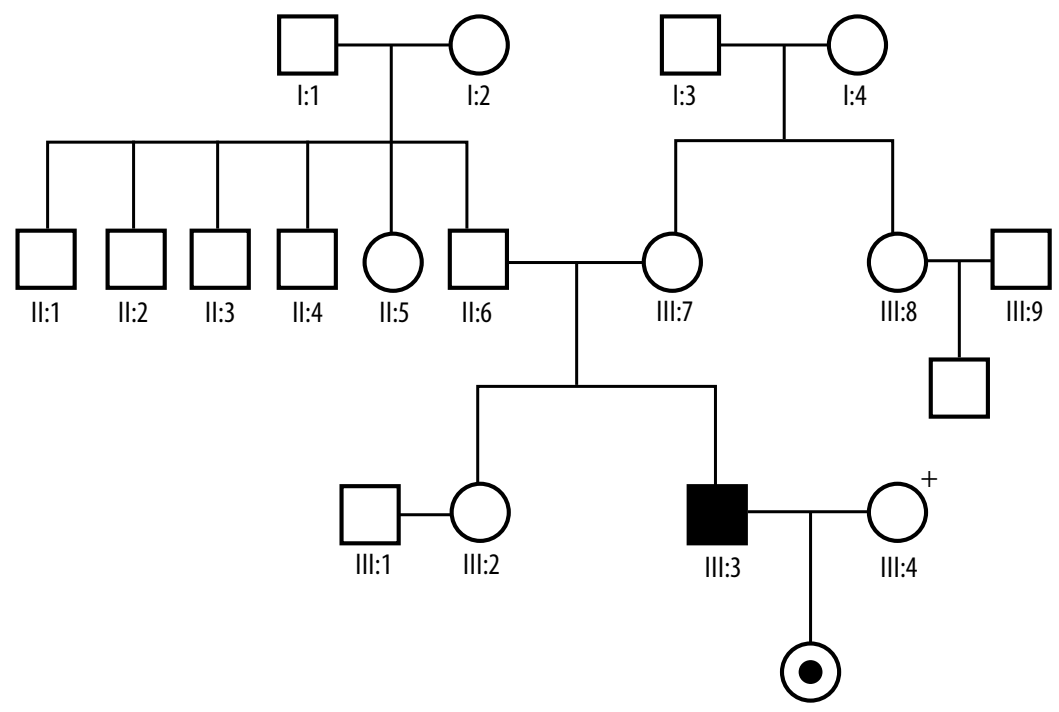

IV:1 
FIGURE 2.

Clinical features from choroideremia-01 family. (a) The fundus pictures of both eyes in patient IV: 7 from family choroideremia-01 (top), lower pictures show the infrared images and spectral-domain optical coherence tomography of both eyes. Note the preservation of inner retinal layers, disruption of outer retinal layers, retinal tubulation (white arrows) \& choroidal thinning. (b) Fundus pictures, fundus autofluorescence and the spectral-domain optical coherence tomography of the asymptomatic mother (III:5). Top -Fundus pictures of the right and left eyes. Bottom right - fundus autofluorescence of the right and left eyes showed the patchy autofluorescence. Lower left - Infrared and spectral-domain optical coherence tomography images of the eyes showing the retinal pigment epithelial irregularities (white arrows)
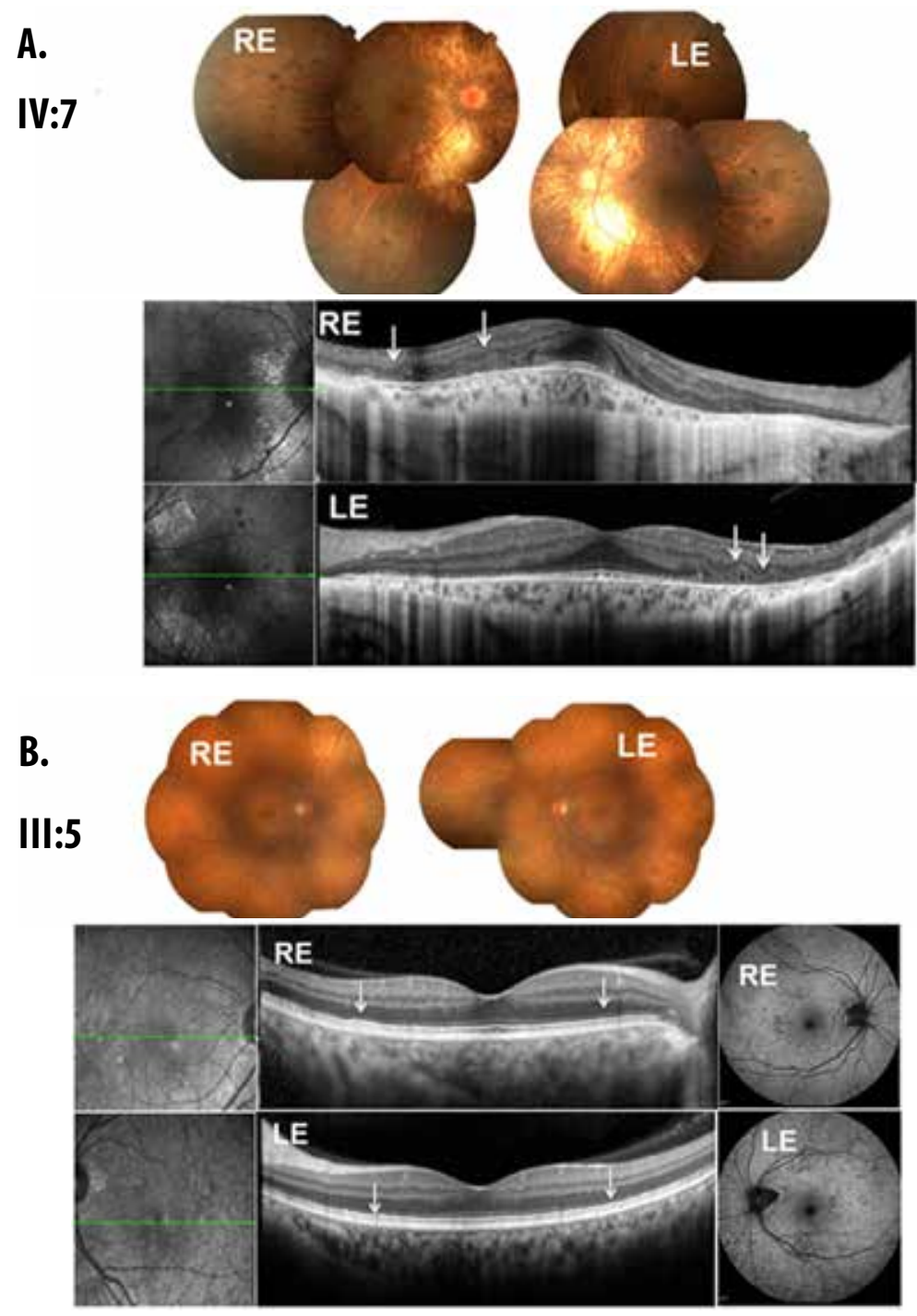
FIGURE 3.

Clinical features from choroideremia -02 family. (a) Clinical details of patient (IIl:3). Top left- FP (Optos). Middlefundus autofluorescence showed a small area of normal AF (white arrows). Lower-spectral-domain optical coherence tomography showed the loss of outer retinal layers, choroidal thinning and preservation of the inner retinal architecture. The LE shows a few cystoid spaces in the macula. Top right: Humphrey visual fields (10-2) showing the remaining extremely small visual fields. (b) Clinical details of the daughter (IV:1). Top- FP, showing the extensive retinal pigment epithelial pigmentation throughout the fundus (white arrows). Lower left - fundus autofluorescence showing the patchy AF. Lower right - infrared and spectral-domain optical coherence tomography pictures showing the retinal pigment epithelial irregularity (yellow arrows).
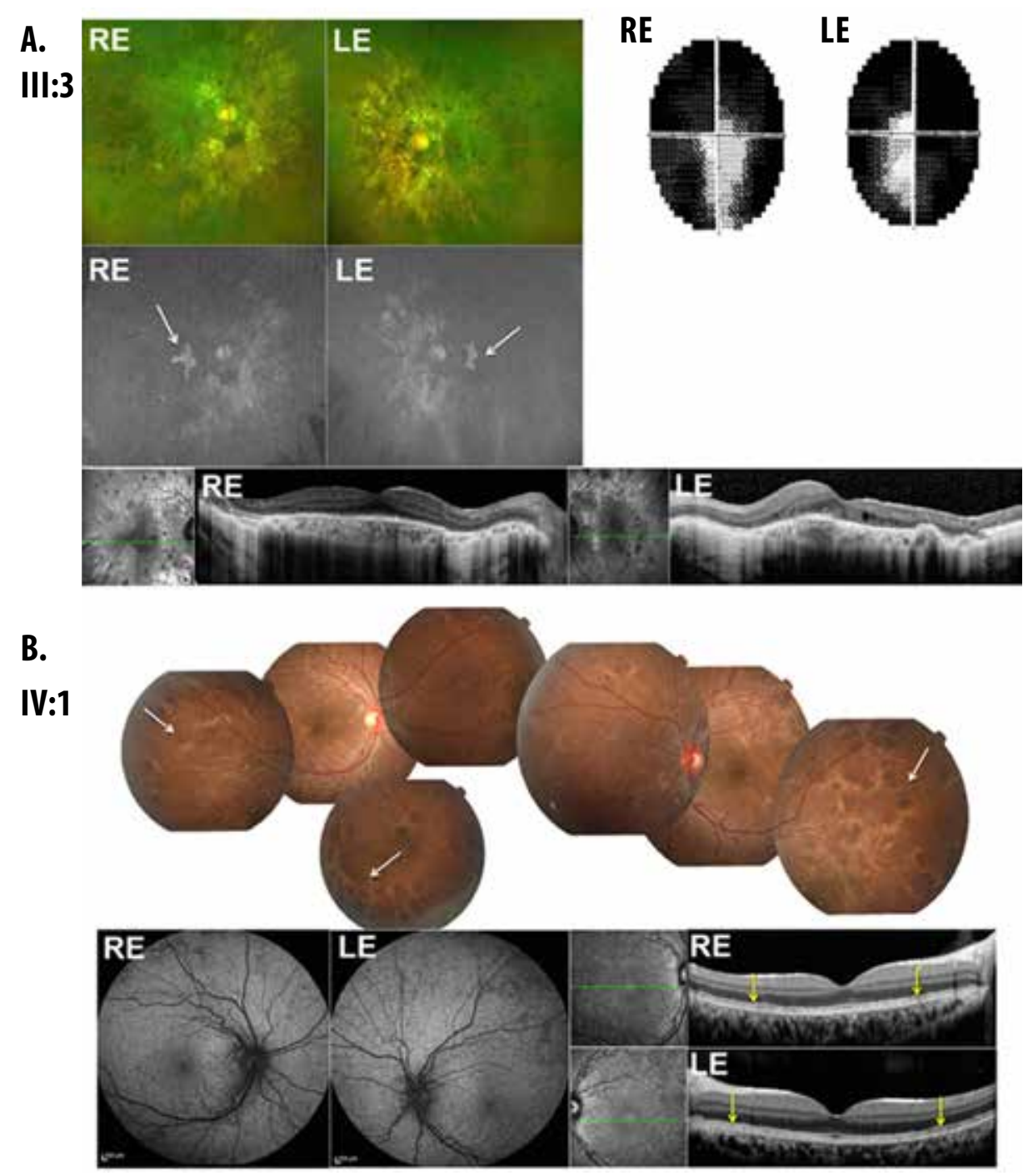


\section{Discussion}

This is the first report to describe the genotype and phenotype findings in patients with Choroideremia from India. Variety of CHM mutations known to cause X-linked inherited or sporadic CHM have been summarized in databases (https://grenada.lumc.nl/LOVD2/ Usher_montpellier/home.php?select_db=CHM, http://www.retina-international.org/ sci-news/databases/mutation-database/chm-mutation/). CHM (REP-1) is ubiquitously expressed, has a vital role in prenylation of Rab proteins, which are essential for vesicle trafficking in endocytic and exocytic pathways. In CHM patients, loss of function of REP-1 due to $\mathrm{CHM}$ mutations may cause progressive degeneration of choroid, photoreceptors and RPE. ${ }^{20,21}$ The clinical features associated with CHM include night blindness, visual field constriction, reduced visual activity and progressive chorioretinal degeneration. Some of these clinical features may overlap with XLRP, one of the more severe forms of RP. ${ }^{22}$ Symtoms of night blindness and constriction of peripheral visual fields are common in both diseases. High myopia and waxy pallor of discs is more commonly seen in RP. Fundus in CHM is characterized by chorioretinal scalloped atrophy initiated from the mid-periphery without affecting the macula till the late stages. In contrast, due to a greater degree of RPE migration seen in RP, bony spicules are commonly seen throughout the fundus. In early stages of XLRP, the choroid is relatively normal in comparison to $\mathrm{CHM}$ where there is extensive chorioretinal degeneration. Carriers of both diseases exhibit characteristic pigmentary alterations in the fundus. Some carriers of XLRP may show golden tapetal-like fundus reflex.

We therefore carried out a genetic analysis using the combination of targeted NGS (panel of gene test) and validation with Sanger sequencing on CHM, RPGR and RP2 genes, the latter two associated with XLRP. High resolution imaging of the choroid is useful to assess the severity and determine the progression of the disease. In both our patients, we noted that photoreceptor degeneration was less severe over the areas of relatively preserved RPE. ${ }^{23}$ The latter observation was further confirmed by OCT findings that retinal lamination and thickness remained remarkably normal in areas in which the RPE was preserved in contrast to generalized RPE and choriocapillaris atrophy. ${ }^{23}$ It has been shown that RPE changes precede retinal degeneration. ${ }^{23,24}$ FAF of both patients (IV:7, III:3) showed a very small island of autofluorescence in the macula, corresponding to the area of functioning RPE. FAF of both the carriers showed speckled autofluorescence throughout the fundus. This corresponds to the mosaic pattern of retinal dysfunction known to occur in carriers of $\mathrm{CHM}_{1}^{25}$ which occurs due to 
Lyonization, the process of random inactivation of X-chromosomes in retinal cells, as in every somatic diploid cell. ${ }^{26}$ In patients with $\mathrm{CHM}$ an abnormal thickening has been noted around the parafoveal region on the OCT early in the second decade of life. Observing a significant alteration in the laminar organization of the retina in patients with $\mathrm{CHM}$, Jacobson et al. hypothesized that these corresponded with remodeling of the retina in CHM. ${ }^{21}$ The earliest abnormality noted was retinal thickening, explained by photoreceptor damage and stress activating Muller cells, the main glial cells in the retina, noted as retinal thickening on the OCT. In advanced stages, there is photoreceptor dysfunction and death and loss of normal retinal lamination. ${ }^{21}$ SDOCT in both male patients (IV:7, III:3) showed the presence of multiple outer retinal tubulations (ORT), significant choroidal thinning and relative preservation of the inner retinal layers. There was a central preservation of the photoreceptor layer and external limiting membrane, possibly corresponding to the remaining visual fields. The choroidal thickness underlying areas of preserved photoreceptors appeared normal, with a sharp reduction in the thickness at the transition zone between normal and atrophic photoreceptors. The tubules were seen prominently at the junction where inner segment/outer segment (IS/OS) loss was noted. There was cystoid spaces at the macula distinguishable from the choroidal tubules only in the left eye of the patient (CHM-02_III:3) in the second family. Zweifel et al. first described the 'ORT' on the SD-OCT. ${ }^{27}$ They hypothesized that these were formed by the rearrangement of the photoreceptor layer in response to retinal injury in many conditions including CHM. ${ }^{27}$ These have been known to develop from the gradual invagination of outer retinal structures at the junction of intact and atrophic retina. ${ }^{28}$ The unaffected individuals (III:5,IV:1) were asymptomatic carriers, showed irregularities in RPE and more prominent outside the macula. The IS/OS junction although undulated, was intact throughout the macula. The RPE showed focal disruption corresponding to the undulations. FAF of the second carrier showed speckled autofluorescence, corresponding to the irregular RPE. In the first family, the patient (IV:7) had a 3'-splice site (c.820-1G $>C$ ) variation at intron 6 of CHM gene associated with ORT. Interestingly, this variation was found in heterozygous state in a female with the family history of $\mathrm{CHM}$, showed detectable level of sub-retinal hemorrhage in the fundus examinations. ${ }^{29}$ Previously, skipping of exon 7 at mRNA level ( $3^{\prime}$-splice site variation) in CHM gene was found in Italian patients with CHM. ${ }^{30}$ This variation (c.820-1G>C) found in $3^{\prime}$-splice acceptor site of intron 6 of CHM can produce altered or shorted REP-1 protein due to alternate splice event that is the likely cause of retinal abnormalities in the patient. Furthermore, a novel variation (c.819+2T>A), at the donor splicing site of intron 6 of the CHM was found in families 
with $\mathrm{CHM}$ associated with various clinical manifestations indicating the intra familial variable expressivity. ${ }^{31}$ In the first family (CHM-01), the proband's mother (III:5) was asymptomatic and showed significant RPE alterations in the fundus examination which is consistent with previous findings. ${ }^{31}$

In the second family (CHM-02), proband (III:3) showed clinical features of advanced CHM such as hyperautofluorescence at the macula, loss of photoreceptors with choroidal thinning with retinal tubules associated with nonsense mutation (p.Ser218X). This mutation leads to premature stop codon at $\mathrm{C}$-terminal region that forms a part of the GDI domain (Rab GDP dissociation inhibitors), ${ }^{32}$ that has functional and regulatory role on post -translational modification (prenylation) and vesicular membrane trafficking. ${ }^{33}$ There was a known mutation (p.Tyr217fs*230) at the adjacent position of Ser 217 that has been implicated in CHM.11 Previously, mutation in C-terminal region of REP-1 protein (p. Ser218Lysfs*13) was reported in CHM patients from Chinese and Italian origins. ${ }^{10,30,34}$

Since the clinical features of CHM overlaps with XLRP, we suggest that the panel of targeted gene sequencing by NGS may be a convenient tool for genetic testing. ${ }^{18}$ Advances in genetherapy and cell based therapy for $\mathrm{CHM}$ holds promise with progress in science. ${ }^{35,36}$

\section{Conclusion}

This is the first report to describe the genotype and phenotype findings in two patients with $\mathrm{CHM}$ from India. Furthermore, these mutations might play a role in pathogenesis consistent with previously reported genotype/phenotype correlations in patients with CHM.. In addition, NGS panel of gene sequencing is an efficient method, that is useful in accurate molecular diagnosis of asymptomatic carriers and helps in genetic counseling.

\section{References}

1. Cremers FP, Sankila EM, Brunsmann F, et al. Deletions in patients with classical Choroideremia vary in size from $45 \mathrm{~kb}$ to several megabases. Am J Hum Genet. 1990;47:622-8.

2. Cremers FP, van de Pol DJ, van Kerkhoff LP, Wiering B, Ropers HH. Cloning of a gene that is rearranged in patients with choroideremia. Nature. 1990;347:674-7.

3. Ohba N, Isashiki, Y. Clinical and genetic features of Choroideremia. Jpn J Ophthalmol. 2000;44:317. 
4. Garcia-Hoyos M, Lorda-Sanchez I, Gomez-Garre P, et al. New type of mutations in three spanish families with Choroideremia. Invest Ophthalmol Vis Sci. 2008;49:131521.

5. Sankila EM, Tolvanen $R$, van den Hurk JA, Cremers FP, de la Chapelle A. Aberrant splicing of the CHM gene is a significant cause of Choroideremia. Nat Genet. 1992;1:109-13.

6. Pereira-Leal JB, Hume AN, Seabra MC. Prenylation of Rab GTPases: molecular mechanisms and involvement in genetic disease. FEBS Lett. 2001;498:197-200.

7. Goody RS, Rak A, Alexandrov K. The structural and mechanistic basis for recycling of Rab proteins between membrane compartments. Cell Mol Life Sci. 2005;62:1657-70.

8. van Bokhoven $H$, van den Hurk JA, Bogerd L, Philippe C, et al. Cloning and characterization of the human a gene. Hum Mol Genet. 1994;3:1041-6.

9. van den Hurk JA, Schwartz M, van Bokhoven H, van de Pol TJ, Bogerd L, Pinckers AJ, et al. Molecular basis of Choroideremia (CHM): mutations involving the Rab escort protein-1(REP-1) gene. Hum Mutat. 1997;9:110-7.

10. McTaggart KE, Tran M, Mah DY, Lai SW, Nesslinger NJ, MacDonald IM. Mutational analysis of patients with the diagnosis of Choroideremia. Hum Mutat. 2002;20:18996.

11. van den Hurk $J A$, van de Pol DJ, Wissinger $B$, et al. Novel types of mutation in the Choroideremia ( $C H M$ ) gene: a full-length $L 1$ insertion and an intronic mutation activating a cryptic exon. Hum Genet. 2003;113:268-75.

12. Shi W, van den Hurk JA, Alamo-Bethencourt $V$, et al. Choroideremia gene product affects trophoblast development and vascularization in mouse extra-embryonic tissues. Dev Biol. 2004;272:53-65.

13. Wavre-Shapton ST, Tolmachova T, Lopes da Silva M, Futter CE, Seabra MC. Conditional ablation of the Choroideremia gene causes age-related changes in mouse retinal pigment epithelium. PLoS One. 2013;8:e57769.

14. Vasireddy V, Mills JA, Gaddameedi R, et al. AAV-mediated gene therapy for Choroideremia: preclinical studies in personalized models. PLoS One. 2013;8:e61396.

15. Tolmachova T, Tolmachov OE, Barnard AR, et al. Functional expression of Rab escort protein 1 following AAV2-mediated gene delivery in the retina of Choroideremia mice and human cells ex vivo. J Mol Med (Berl). 2013;91:825-37.

16. Marmor MF, Fulton AB, Holder GE, Miyake Y, Brigell M, Bach M. ISCEV Standard for full-field clinical electroretinography (2008 update). Doc Ophthalmol (2009) 118:69-77

17. Miller SA, Dykes DD, Polesky HF. A simple salting out procedure for extracting DNA from human nucleated cells. Nucleic Acids Res. 1988;16:1215. 
18. Battu R, Verma A, Hariharan R, et al. Identification of Novel Mutations in ABCA4 Gene: Clinical and Genetic Analysis of Indian Patients with Stargardt Disease. Biomed Res Int. 2015;2015:940864.

19. Furgoch MJ, Mewes-Ares J, Radziwon A, Macdonald IM. Molecular genetic diagnostic techniques in Choroideremia. Mol Vis. 2014;20:535-44.

20. Boye SE, Boye SL, Lewin AS, Hauswirth WW. A comprehensive review of retinal gene therapy. Mol Ther. 2013;21:509-19.

21. Jacobson SG, Cideciyan AV, Sumaroka A, et al. Remodeling of the human retina in Choroideremia: rab escort protein 1 (REP-1) mutations. Invest Ophthalmol Vis Sci. 2006;47:4113-20.

22. MacDonald IM, Hume S, Chan S, Seabra MC. Choroideremia. In Gene Reviews. Pagon RA, Adam MP, Ardinger HH, et al., editor. Seattle (WA): University of Washington; 2003.

23. MacDonald IM, Russell L, Chan CC. Choroideremia: new findings from ocular pathology and review of recent literature. Surv Ophthalmol. 2009;54:401-7.

24. Syed R, Sundquist SM, Ratnam K, et al. High-resolution images of retinal structure in patients with choroideremia. Invest Ophthalmol Vis Sci. 2013;54:950-61.

25. Rudolph G, Preising M, Kalpadakis P, Haritoglou C, Lang GE, Lorenz B. Phenotypic variability in three carriers from a family with Choroideremia and a frameshift mutation 1388delCCinsG in the REP-1 gene. Ophthalmic Genet. 2003;24:203-14.

26. Lyon MF. Gene action in the X-chromosome of the mouse (Mus musculus L.). Nature. 1961;190:372-3.

27. Zweifel SA, Engelbert M, Laud K, Margolis R, Spaide RF, Freund KB. Outer retinal tubulation: a novel optical coherence tomography finding. Arch Ophthalmol. 2009;127:1596-602.

28. Goldberg NR, Greenberg JP, Laud K, Tsang S, Freund, KB. Outer retinal tubulation in degenerative retinal disorders. Retina. 2013;33:1871-6.

29. Potter MJ, Wong E, Szabo SM, McTaggart KE. Clinical findings in a carrier of a new mutation in the Choroideremia gene. Ophthalmology. 2004;111:1905-9.

30. Esposito G, De Falco F, Tinto $N$, et al. Comprehensive mutation analysis (20 families) of the Choroideremia gene reveals a missense variant that prevents the binding of REP 1 with Rab geranylgeranyl transferase. Hum Mutat. 2011;32:1460-9.

31. Contestabile MT, Piane M, Cascone NC, et al. Clinical and genetic studies in a family with a new splice-site mutation in the Choroideremia gene. Mol Vis. 2014;20:325-33.

32. Alexandrov K, Horiuchi H, Steele-Mortimer O, Seabra MC, Zerial M. Rab escort protein-1 is a multifunctional protein that accompanies newly prenylated rab proteins to their target membranes. EMBO J. 1994;13:5262-73. 
33. Nishimura N, Goji J, Nakamura H, Orita S, Takai Y, Sano K. Cloning of a brain-type isoform of human Rab GDI and its expression in human neuroblastoma cell lines and tumor specimens. Cancer Res. 1995;55:5445-50.

34. Yip SP, Cheung TS, Chu MY, et al. Novel truncating mutations of the CHM gene in Chinese patients with Choroideremia. Mol Vis. 2007;13:2183-93.

35. Anand V, Barral DC, Zeng Y, et al. Gene therapy for Choroideremia: in vitro rescue mediated by recombinant adenovirus. Vision Res. 2003;43:919-26.

36. Abe T, Yoshida M, Yoshioka Y, et al. Iris pigment epithelial cell transplantation for degenerative retinal diseases. Prog Retin Eye Res. 2007;26:302-21. 


\section{CHALLENGES OF MANAGING RETINAL DYSTROPHIES: \\ AN EXPERIENCE FROM SOUTH INDIA}

Rajani Battu, Ashwin Mallipatna, Niby Jacob Elackatt, Tos TJM Berendschot, Jan SAG Schouten, Carroll AB Webers OPHTHALMIC GENET. 2018:39(1); 1-3 

Generally considered as'rare' or'orphan' diseases, retinal dystrophies are highly prevalent in India, in part because of the high rate of consanguinity. For example, the prevalence of retinitis pigmentosa in the US is estimated to be 1 in $3500,{ }^{1}$ compared to an estimate of 1 in 372-930 in southern India. ${ }^{2}$ This is similar to the situation in other Asian countries, like Pakistan. ${ }^{3}$ As opposed to Western countries, the clinical science of eye genetics and the practice of genetic counseling are still developing specialties in India. Physicians/ genetic counselors who are specialized or trained to deal with these conditions are scarce. Molecular genetic testing is either inaccessible or unaffordable to the majority of patients. Consanguinity and arranged marriages are an accepted norm, especially in southern India. In addition, opportunities for rehabilitation of severely visually impaired person are limited. Consequently, managing patients with retinal dystrophies presents specific clinical and psycho-social challenges in India.

\section{Challenges}

\section{Health Economics}

Although the millennium has witnessed some remarkable progress in public health in India, the percentage of India's national budget allocated to the health sector remains one of the lowest in the world. ${ }^{4}$ The country continues to battle major health problems; it has one of the largest numbers of infant deaths, maternal deaths and tuberculosis in the world.

The World Health Organization's 2000 global healthcare profile ranked India's healthcare system $112^{\text {th }}$ out of 190 countries. The majority of the Indian population lives in rural areas, with extremely limited access to quality health care. Only 3-5\% of Indians are covered by health care insurance, leading to a high out-of-pocket expenditure to the patients. ${ }^{4}$

India has a whopping 70 million people affected with rare diseases, including the spectrum of inherited cancers, congenital malformations, storage diseases and retinal degenerative diseases. ${ }^{5}$ Considering an average prevalence of retinitis pigmentosa of 1 in 1000, and given India's burgeoning population of over 1.3 billion, this equates to over a million people with RP alone. While the country struggles for basic healthcare, patients with rare diseases do not get the attention they need. 


\section{Diagnostic Challenges}

Accurate diagnosis of an inherited retinal disease (IRD) is a complex process. Many IRDs present with similar initial symptoms, and heterogeneity of phenotype and genotype is the rule. The patient has to therefore undergo a number of ophthalmic investigations including perimetry, optical coherence tomography (OCT) fundus autofluorescence photography and electroretinograpy before a tentative clinical diagnosis is reached. Furthermore, some of these tests need to be repeated at regular intervals to monitor disease progression. Patients with syndromic diseases such as Bardet-Biedl syndrome and others require consultation with multiple medical specialists. Considering that majority of the Indian population is not covered by health insurance, this leads to a significant burden on their finances and time. In a consanguineous population like that of southern India, it is imperative that extended family members are examined as well, leading to increasing financial burden.

\section{Lack of Trained Personnel}

Lack of trained genetic counselors is a huge challenge in India. It is estimated that there are 50 medical/clinical geneticists and about 29 genetic counselors in India working across 50 centers in the country. ${ }^{6}$ Very few of these centers offer genetic counseling for IRDs.

There are less than a dozen tertiary referral ophthalmic centers across the country that offer comprehensive genetic services with clinicians trained in ophthalmic genetics, genetic counselors, molecular genetic testing and specialists in low vision rehabilitation. General ophthalmologists lack awareness about IRDs and their management; therefore patients are neither managed appropriately nor referred to specialized genetic centers. In most situations, the responsibility of breaking the news, discussing the genetic reports (when available), and offering counseling rests mostly with the primary physician. Many a times, these patients are examined in the setting of busy outpatient clinics, and allocating the necessary time for these patients is a major challenge to the physician.

\section{Lack of Infrastructure}

Genetic testing is a critical tool in the diagnosis of an IRD; it is useful in determining the inheritance pattern, clarifying diagnosis, attempting to clarify its prognosis and optimizing clinical care. Affordability and accessibility to these tests are difficult to the majority of patients in India. Even when available, many families opt out of these investigations in the absence of an immediate treatment prospect. 
Low-vision rehabilitation, which offers a ray of hope to patients with IRDs and failing vision, is still in its infancy in most eye hospitals across India. Very few ophthalmic hospitals specialized low vision rehabilitation departments and the necessary equipment to offer to patients. Patient support groups, which form an integral part of rehabilitation in other countries, are almost nonexistent for patients and families with IRDs in India. Consequently, access to information about practical difficulties faced by the patients, treatment options, and psychosocial support structure is insufficient.

\section{Consanguinity}

Consanguineous marriages continue to be a common tradition in the southern part of the country. Parental consanguinity was reported in $24.7 \%$ of rural subjects and $32.9 \%$ of urban subjects in the Andhra Pradesh Eye Disease Study. Parents often find it hard to accept the higher risk that consanguinity poses in the transmission of retinal dystrophies. One of the curious dilemmas that parents of children with retinal dystrophies face occurs when these children attain the age of marriage. In a society where arranged marriages are still very common, parents find it easier to find a partner for their affected child within their own families, thus continuing to propagate consanguinity.

Published reports of IRDs in India show that over $75 \%$ of patients with RP are transmitted in an autosomal recessive fashion. ${ }^{8}$ In a study of 400 cases of RP, Chakrabarti et al. showed that $75 \%$ of cases were autosomal recessive, $10 \%$ autosomal dominant and $1 \%$ X-linked. The inheritance pattern was not clear in 14\%. They attributed the high frequency of recessive cases to inbreeding and consanguinity.

\section{Addressing These Challenges}

Given the multiple stakeholders who are involved: patients, physicians, genetic counselors, diagnostic laboratories, rehabilitation units and the government, managing retinal dystrophies becomes a very complex process.

Considering that there are no current practice guidelines and standards to impart competent genetic counseling services, training genetic counselors is the most urgent important step in addressing these challenges in India. More centers that can train genetic counselors across the nation need to be identified, and counselors need to be part of the clinical case management. Physicians need to be made aware of the complexities of the disease spectrum. Instead of the current practice in which the physician manages most aspects of the disease, a team approach needs to be cultivated. Counseling is best done when patient/parents communicate directly with the team, involving all treating 
physicians, genetic counselors and social workers. Although sporadic at present, teleophthalmology may be an effective way of providing genetic counseling to patients in remote parts of the country.

Indians in South Asia represent a sixth of the world's population and are a racially, geographically, and genetically diverse people. With endogamous and consanguineous marriages being common, the frequency of founder mutations and burden of heritable genetic disorders is high. ${ }^{9}$ Molecular genetic testing therefore needs be tailored to be accessible to a large number of patients and at the same time allow identification of new founder mutations. Although creating diagnostic panels reduce the cost of genetic testing initlally, whole exome sequencing may eventually be superior to gene panels, as the cost of genetic testing reduces globally. Also, genetic studies from neighboring countries like Pakistan where consanguinity is equally high may help design screening platforms in India. ${ }^{10}$

Nearly $70 \%$ of the Indian population lives in rural areas. Indian society is 'collectivistic' in that it promotes co-operation and interdependence among family members. ${ }^{11}$ The joint family system that still exists in rural India is far more involved in the caring of its members, and share the burden of visual disability amongst its members. To a visually disabled person, family support becomes the backbone of everyday existence. Given this support that joint family systems confer to patients, it is interesting that patients from rural areas accept bad news with more equanimity than those with an urban background. This also makes it difficult to dissuade parents from arranging consanguineous marriages. Appropriate genetic and social counseling with community education is necessary to alert patients about the ill- effects of consanguineous unions.

Establishing effective state-of-the art low vision rehabilitation services along with genetic clinics that manage patients with IRDs is imperative in providing customized solutions to the individual patient. Appropriate visual rehabilitation allows patients to be better integrated into the normal society. But these visual aids need be customdesigned in most patients, and therefore need dedicated and trained optometrists/ staff to address this.

Creating patient support groups goes a long way in enabling patients manage their day-to-day life, and evolve psychosocial support structures and integrating patients into society. While support groups for each disease, and sometimes, specific mutations 
are common in many countries, this is an evolving concept in India. Individuals within the patient community need to be identified and encouraged to form and lead support groups.

Centers of excellence that address the needs of patients with IRDs need to be developed and will become pivotal to the diagnosis and management of these individuals. The Organization for Rare Diseases India (ORDI) has been formed to address the unmet needs in this area. ${ }^{12} \mathrm{ORDI}$ is an umbrella patient support organization that aims to help early diagnosis, provide genetic counseling and inform families about treatment options and clinical trials, collect epidemiological data and catalyze research in genetic diseases. There is a burning need for a center dedicated to IRDs alone, which may be formed with collaboration with a similar center in the Western countries.

\section{Conclusions}

The number of people with IRDs is significantly higher in India than many developed countries. Patients face many challenges in obtaining appropriate diagnosis, management, and support facilities. There is a lack of awareness and public policies that pertain to this group of patients. With increasing awareness among patients and the progress in treatment of these conditions around the world, it is imperative that centers of excellence for genetic eye diseases are established, personnel trained, and low visual rehabilitation services expanded. Efforts should also be made to create support groups and work towards creation of public policies that benefit patients with IRDs. The spectrum of mutations and genes commonly involved in IRDs in the Indian population need to be established. This can advance our understanding of the disease mechanisms, thus paving way for research into treatment options and early participation in clinical trials.

\section{References}

1. Bunker CH, Berson EL, Bromley WC et al. Prevalence of retinitis pigmentosa in Maine. Am J Ophthalmol. 1984;97(3):357-365.

2. Sen P, Bhargava A, George $R$, et al. Prevalence of retinitis pigmentosa in South Indian population aged above 40 years. Ophthalmic Epidemiol. 2008;15(4):279- 281.

3. Adhi Ml, Ahmed J. Frequency and clinical presentation of retinal dystrophies: a hospital based study. Pak J Ophthalmol 2002;18(4): 106-110 
4. Ahlin T, Nichter M, Pillai G. Health insurance in India: what do we know and why is ethnographic research needed. Anthropology \& Medicine. 2016; 23:1,102-124.

5. Verma IC. Burden of genetic disorders in India. Indian Journal of Pediatrics.2001; 67:893-898. Erratum in: Indian Journal of Pediatrics (2001). 68, 25.

6. Singh JR, Singh AR and Singh AR. Directory of human genetic services in India-2007. Int J Hum Genet. 2010;10:187-192.

7. Nirmalan PK, Krishnaiah S, Nutheti R, et al. Consanguinity and eye diseases with a potential genetic etiology. Data from a prevalence study in Andhra Pradesh, India. Ophthalmic Epidemiol. 2006;13(1):7-13.

8. Chakrabarti S, Sarhadi VK, Singh D, et al. Clinical genetic analysis of retinitis pigmentosa in Indian population. Indian J Human Gent. 2001; 1(2): 133-137.

9. Ankala A, Tamhankar PM, Valencia CA, et al. Clinical applications and implications of common and founder mutations in Indian subpopulations. Hum Mutat.2015; 36:1-10.

10. Khan MI, Azam M, Ajmal M, et al. The molecular basis of retinal dystrophies in Pakistan. Genes 2014; 5(1): 176-195

11. Chadda RK and Deb KS. Indian family systems, collectivistic society and psychotherapy. Indian J Psychiatry. 2013;55:299-309.

12. Rajasimha HK, Shirol PB, Ramamoorthy $P$, et al. Organization for rare diseases India (ORDI) - addressing the challenges and opportunities for the Indian rare diseases' community. Genet Res (Camb). 2014;96:1-10. 


\section{METHODS TO DETECT PROGRESSION IN RETINITIS PIGMENTOSA: A REVIEW}

Rajani Battu, Jan SAG Schouten, Tos TJM Berendschot and Carroll AB Webers

IN SUBMISSION 



\section{Abstract}

AIM: Retinitis pigmentosa is a slowly progressive genetic disease with several treatments under investigation either to treat the disease or halt its progression. The methodologies to assess progression include conventional methods like kinetic perimetry, automated Humphrey perimetry, electroretinography and new methods like fundus autofluorescence, optical coherence tomography, microperimetry and adaptive optics. The aim of this study is to review data from literature to assess the methods used to assess progression of the disease.

METHODS: Publications up to March 2018 that assessed progression of RP using two or more methods were studied. These included randomized clinical trials conducted for the medical treatment of RP and publications that studied the progression of RP.

RESULTS: INinety studies were included. The relevant outcome measures included visual field assessment (VF), optical coherence tomography (OCT), fundus autofluorescence (FAF), full field electroretinogram (ERG), multifocal electoretinogram (mfERG), microperimetry (MP) and adaptive optics (AO). Each study had included multiple outcome measures to assess the progression; all were able to assess progression and correlated with other methods. Apart from the visual fields measurement, several studies that correlate changes on the FAF/OCT/MP with ERG and visual fields are available. Newer data continue to emerge from AO and OCT angiography (OCTA) to assess structural changes in detail.

CONCLUSIONS: Several old and new methods to assess progression in RP are now available. Although several methods to assess progression correlate, the correlation is not perfect. This suggests that multiple modalities need to be used simultaneously to assess progression and understand mechanism of progression and its influence on daily activities in these patients. 


\section{Introduction}

Retinitis Pigmentosa (RP) is a group of disorders, characterized by progressive degeneration of rod and cone photoreceptors. It is a leading cause of visual disability, with a prevalence of 1 in 4000 in the US, and 1 in 1000 in countries like India and China..$^{1-3}$ Apart from a recently approved drug for RPE65 mutation, there is no effective treatment that can be offered to a patient, although several clinical trials are underway. ${ }^{4,5} \mathrm{Given}$ the explosion of clinical trials and possible therapies on the horizon, it is necessary to identify appropriate end points to monitor response to these trials.

Progression in RP has traditionally been monitored in the past by assessing visual fields and full-field electroretinography. This paper is an attempt to summarize the various methods that have been used traditionally and recently, to understand the progression of RP.

\section{RP and Visual Fields}

Monitoring changes in the visual fields has been the gold standard of assessing the progression of RP over time. For a very long time, this was done using Goldmann's kinetic perimetry.

Kinetic perimetry is the oldest technique of measurement known. Goldmann kinetic perimetry involves projection of light into a white bowl with a standardized background light intensity and a stimulus moved from the nonseeing into the seeing area. At the end of the test, the points noted are connected by lines to form the smooth boundaries of the visual field, the isopters. Areas of decreased sensitivity (scotoma) are mapped by moving a target from the seeing to non-seeing areas in different directions. The final output is a series of isopters. ${ }^{6}$

Automated perimetry maps the visual field with the aid of a computer. These are static perimeters that present patients with non-moving stimuli of varying sizes and intensities on a uniform background. The illumination, measured in apostilbs or decibels is an indication of stimulus intensity. Static perimeters measure the patient's response in the central and peripheral visual fields, varying from $10^{\circ}, 24^{\circ}, 30^{\circ}$ and $60^{\circ}$. The most frequently used standard automated perimeters are Humphrey and Octopus. 
Both types of perimeters have been used in patients with Retinitis Pigmentosa. Gonin suggested that visual field changes began as a partial or complete ring scotoma in the midperiphery that gradually progressed, leaving only a markedly constricted central field. ${ }^{7}$

Grover et al. in 1997 attempted to quantify the rate of visual field loss in patients with RP and correlate it with different phenotypes on using Goldmann perimetry. ${ }^{8}$ They found that the average half-life (time over which half of the remaining field area would be lost) of the visual field area was 7.3 years for target Ve4 and 6.8 years for target $11_{4 \mathrm{e}}$, which were not statistically different $(P=0.16)$. Autosomal recessive cases had a higher rate of visual field loss compared to either isolated or autosomal dominant cases. ${ }^{9}$ Berson et al. followed up patients with RP over a 3-year period and reported that patients, on an average lost $4.6 \%$ of the remaining visual field area per year and lost $2.4 \%$ of the remaining visual field diameter per year. ${ }^{10}$

Considering that changes in the central $10 \mathrm{deg}$ is most important for patients with moderate to severe visual field loss, Hirakawa et al. further showed significant deterioration in the central 10-deg visual fields in patients with RP using an automated static perimeter. ${ }^{11}$ They also demonstrated that the univariate linear regression of the mean deviation (MD) on the Humphrey perimeter was a useful parameter to monitor progression, and which assesses the sensitivity distribution in the macular area in eyes affected by RP.

Swanson et al. assessed the effect of the stimulus on static visual fields in patients with RP. ${ }^{12}$ They concluded that in regions of visual fields that were already damaged by RP, increase in stimulus size from III to V produced significant improvements in perimetric sensitivity. They found that size III may be more useful than size V for detecting abnormalities in visual fields, while size $\mathrm{V}$ may be more useful to detect progression.

\section{$\mathrm{RP}$ and Electroretinography}

Objective measurement of electroretinography (ERG) has been used as a tool to assess the function of the retina in RP. These include full field ERG to assess the overall retinal function and pattern electroretinogram, multifocal electroretinogram (mfERG) and the multifocal visual evoked potential (mfVEP) to assess the central function.

In one of the earliest studies on ERG in RP, Berson et al. in 1985 studied 94 patients with RP over 3 consecutive years. On an average, patients lost $16 \%$ to $18.5 \%$ of remaining electroretinographic amplitude per year and $4.6 \%$ of remaining visual field area per year. ${ }^{10}$ 
Granse et al. in 2004 attempted to study the residual visual function in 14 patients with advanced RP using different electrophysiological studies- full field ERG, mfERG and mfVEP. ${ }^{13}$ They found that the full field ERGs was reduced in all patients and concluded that $\mathrm{mfERG}$ and $\mathrm{mfVEP}$ may be useful for evaluating the residual retinal function.

Nagy et al. did a long-term follow up of patients with RP using mfERG. ${ }^{14}$ They studied 23 patients with RP over a 10-year period using psychophysical studies and mfERG. They concluded that mfERG complemented psychophysical methods like visual fields and could be an objective outcome measure following treatment.

Falsini et al. evaluated long-term changes of central cone- mediated function in 43 patients with RP over an average of 9.3 years by recording focal electroretinograms (fERG). ${ }^{15}$ Mean yearly decay rate of central fERG was $5.6 \%$. They concluded that the decline of central cone function was significantly slower than global cone function decline in RP.

\section{$\mathrm{RP}$ and $\mathrm{OCT}$}

With progress in technology arrived optical coherence tomography (OCT), an objective way to measure retinal structure. OCT is a non-contact, non-invasive technique that provides very high resolution cross sectional images of the retina. While the axial resolution of time-domain OCTs was 8-10 microns, newer spectral domain OCTs (SD-OCT) can achieve 5-8 micron axial resolution. Studies with time domain OCT showed good quantitative agreement between the extent of local field loss and the thickness of the outer nuclear layer. ${ }^{16}$ As SD-OCTs came into vogue, it became possible to measure the thickness of the outer segment (OS) since the inner segment/outer segment (IS/OS) line is a very clearly demarcated.

Witkin et al. studied photoreceptor integrity in patients with RP using ultra high resolution OCT. ${ }^{17}$ They measured the central foveal thickness (CFT) and defined 'foveal photoreceptor outer segment/pigment epithelium thickness (FOSPET)'. FOSPET was defined as the distance between the inner border of the highly reflective line representing the photoreceptor inner/outer segment junction and the outer border of the RPE, measured at the thinnest point of the fovea. They found that FOSPET was statistically thinner in patients with RP compared with normal subjects, and a thinner FOSPET correlated with decreased visual acuity.

Rangaswamy et al. found that the thickness of the outer segment on SD-OCT decreased with local field loss and became undetectable in locations where visual field sensitivity 
loss was worse than $-10 \mathrm{~dB} \cdot{ }^{18} \mathrm{~A}$ study of the transition zone from regions of normal to abnormal vision reported that the earliest sign of damage in RP was a thinning of the OS layer, followed by a decrease in ONL thickness, and then a disappearance of the OS layer. ${ }^{19}$

Fischer et al. identified that the distance between the foveal center and the loss of IS/ OS signal correlated with distance to the edge of the visual field as measured with Goldmann perimetry. ${ }^{20} \mathrm{Hood}$ et al. attempted to derive the boundaries of visual fields from the OCT scans of patients, in whom the central visual fields were relatively well preserved. ${ }^{21}$ They confirmed that the IS/OS line on the SD-OCT disappeared when the visual field sensitivity was less than $-8 \mathrm{~dB}$. They concluded that the IS/OS contour showed promise as a structural measure of progression of the disease and could be used as a marker following treatment.

Birch et al. attempted using the inner segment ellipsoid zone (EZ; ie, inner/outer segment border) to study the progression of a cohort with $\mathrm{x}$-linked RP. ${ }^{22}$ They found that the mean rate of decline in EZ width (7\%) translates into a mean rate of change of $13 \%$ for the equivalent area of functioning retina. This rate of change is consistent with that reported for visual fields and full-field electroretinograms. They concluded that the validity of EZ width as an outcome measure in prospective clinical trials in RP.

Mitamura et al. further suggested that the external limiting membrane (ELM), IS/OS and cone outer segment tips (COST) are all shortened with progression of RP. ${ }^{23}$ The line length is longest in the ELM, followed by the IS/OS, and COST, suggesting that retinal layer becomes disorganized first at the COST, followed by the IS/OS and finally the ELM. This finding was consistent with the previous report that the earliest histopathological change in RP is a shortening of the photoreceptor outer segments.

In a further study to their previous one, ${ }^{21}$ Ramachandran emphasized the importance of measuring the ellipsoid zone width to assess progression on horizontal and vertical scans in patients with RP except those with advanced RP. ${ }^{24}$ However they emphasized the limitation of the study in treatment trials that aim to recover vision, as we do not know the extent to which recovery of the EZ band tracks recovery of visual sensitivity.

In addition to the EZ zone, studies showed that the thickness of the outer segment as demonstrated on the OCT was a very important correlate with traditional measures of visual function in RP, such as multifocal ERG, full field ERG and visual field sensitivity..$^{11,22,25,26}$ 
Wen et al. studied the response to oral administration of QLT091001, a synthetic 9-cis-retinoid, in patients with RP and LCA. ${ }^{26}$ They found that among responders, the average $O S$ thickness measures in the central 20 degrees were significantly associated with global visual field change measured by GVF, which includes not only central field, but also field in the periphery. They concluded that SD-OCT thickness in the central retina may be useful for predicting the visual field response in the peripheral retina to QLT091001.

In addition to the outer retinal thickness, OCT measurements of the retinal nerve fiber layer (RNFL) were made, but these measurements have not been shown to be consistent.

\section{$\mathrm{RP}$ and FAF}

Scanning laser ophthalmoscope allowed the measurement of fundus autofluorescence (FAF), a quick and noninvasive imaging tool that serves as a surrogate to retinal pigment epithelial function in RP. Robson et al. showed a high correlation between pattern ERG, an established technique to assess central function in RP with FAF, concluding that FAF is a valuable parameter in monitoring patients with $\mathrm{RP}^{27}$ In a subsequent longitudinal study, Robson et al. demonstrated a progressive constriction of the FAF ring accompanied by worsening macular function in patients with RP. ${ }^{28}$ Lima et al. compared OCT findings with those of FAF in patients with RP and found that disruption of the IS/OS junction and a decrease in the outer retinal thickness was seen within the central hypofluorescent ring. ${ }^{29}$ Wakabayashi et al. found normal FAF surrounded by a ring of increased FAF at the macular area in these patients. ${ }^{30}$ The area of normal FAF corresponded with preserved IS/OS junction on the SD-OCT while there was a loss of IS/OS and ELM outside the FAF. The ring of increased FAF demarcated the border between the central retina with preservation of the IS/OS junction and ELM, and the adjacent eccentric retina with loss of these bands.

\section{RP and Microperimetry}

Further progress in technology brought the microperimeter (or the fundus-related perimeter), where perimetry is performed with simultaneous viewing of the fundus. This allows observation of the point on the retina that is being stimulated, making it possible to compare retinal morphology to function. Acton et al. compared the difference in the visual field defects measured between the Humphrey visual fields and MP-1 microperimeter. ${ }^{31}$ They observed that there was a difference in the background 
luminance levels and the dynamic range between the two devices. The HFA detected fewer and shallower defects in patients with RP.They hypothesized that in photoreceptor diseases like RP, increment threshold measurements depend on adaptation levels, unlike diseases like glaucoma that primarily involve the inner retina and postreceptoral sites, and in which increment thresholds are independent of adaptation levels. Also, the narrower range of the MP-1 may limit the ability to follow patients whose sensitivities lie beyond the dynamic range of the microperimeter. They concluded that although the examinations on the MP-1 were longer due to the increased adaptation time to the dimmer background and increased test duration, the fundus tracking, ability to quantify the fixation stability and co-registration with the fundus image make the MP-1 a useful device in patients with RP.

Aizwa et al. studied the correlation between FAF and MP in patients with RP over a 2-year period. ${ }^{32}$ They found that the diameter and area of the FAF ring and the length of the IS/OS line decreased significantly during the follow up period. The decrease in the FAF ring diameter was significantly correlated with decrease in retinal sensitivity on the MP-1. They concluded that a progressive constriction of the autofluorescent ring might reflect morphological changes of the photoreceptors and worsening of visual function in $\mathrm{RP}$, thus making it an important parameter in monitoring these patients.

Lenassi et al. also found a high correlation between retinal sensitivity on the MP-1 and the outer retinal thickness on the SD-OCT. The FAF ring correlated with areas of impaired integrity of the photoreceptor layer on the SD-OCT. ${ }^{33}$ Battu et al. attempted to compare the retinal sensitivity on the MAIA (Macular Integrity Assessment), another form of microperimetry with structural changes on the SD-OCT. ${ }^{34}$ Their results showed that the retinal sensitivity on the MAIA correlated with the total retinal and outer retinal thickness on the SD-OCT.

Igarashi et al. investigated the test-retest rreproducibility of visual fields measured with the MP-3 microperimeter. They measured the visual fields twice both with MP-3 and HVF, using the 10-2 test grid pattern in both perimeters. They found that the mean sensitivity measured in the HFA 10-2 was significantly higher than that measured in the MP-3. ${ }^{35}$

\section{RP and Adaptive Optics}

Adaptive Optics $(A O)$ is a relatively new tool that provides excellent lateral resolution, thus enabling visualization of the photoreceptors, blood vessels and details of the 
optic nerve head. ${ }^{36,37}$ Duncan et al. showed that adaptive optics scanning laser ophthalmoscopy (AOSLO) images could be used to study macular cones with high resolution in patients with degenerative diseases of the retina. ${ }^{38}$ Makiyama et al. showed that the cone density was decreased and the regular arrangement of the cone mosaic was altered even in the presence of good visual acuity in patients with $\mathrm{RP}^{39}$ Tojo et al. correlated the macular changes noted on AO with those on OCT and FAF. $^{40}$ The cone densities were reduced at the macula, especially at the parafoveal ring of on high-density FAF and at the border of IS/OS line on the OCT. Menghini et al. found a significant correlation between outer nuclear layer (ONL) thickness and cone density in patients with RP. ${ }^{41}$ The ONL contains rod and cone photoreceptor nuclei and studying these can be a marker for photoreceptor survival in RP.

In a study of eleven patients with RP, Sun et al. correlated the changes on OCT and AO to assess the cone photoreceptor structure. ${ }^{42}$ They concluded that foveal cone density was reduced by $38 \%$ before changes were noted on the OCT or visual function changes were noted; and therefore AOSLO imaging was an early measure of RP changes in RP.

OCT angiography (OCTA) and retinal oximetry are recent modalities that are still being investigated. ${ }^{43,44}$ They may provide further markers to assess progression in RP. These are particularly promising given that vascular attenuation is an early and definite clinical finding in RP. OCTA is a new, noninvasive way to assess changes in blood flow at the macula. Microvascular changes in both the superficial and deep vessels of the parafoveal plexus have been noted. ${ }^{43,45}$

There are several clinical trials in progress/ on the horizon to treat patients with inherited retinal dystrophies. Although several tests like the visual fields, ERG, OCT and $\mathrm{AO}$ provide evidence of progression and serve as surrogate markers for this, it is important to understand that a multimodal assessment is necessary to define important parameters of retinal structure and function in patients with advanced vision loss. In spite of these, tests to define clinical end points in a therapeutic trial remains a major challenge, considering the advanced disease these patients have. Recently, novel endpoints like multi-luminance mobility test that track functional vision changes or spatially resolved functional assessment of rods and cones are being tested out in therapeutic trials. ${ }^{46,47}$

In conclusion, many current investigations for RP help identify progression of the disease. However an accurate co-registration of multiple modalities increases the power of structure-function correlation. With further progress in technology and 
the implementation of multiple therapeutic clinical trials, it is possible that we identify techniques/technologies to identify end points that are easy to assess. The search for an ideal imaging biomarker that can identify and measure progression of RP over a short time continues. Until then, a multimodal assessment that evaluates both structural and functional changes will be the best way to study mechanisms of progression and effects of new treatments.

\section{References}

1. Bunker CH, Berson EL, Bromley WC, Hayes RP, Roderick TH. Prevalence of retinitis pigmentosa in Maine. American journal of ophthalmology 1984;97:357-65.

2. Nangia $V$, Jonas JB, Khare A, Sinha A. Prevalence of retinitis pigmentosa in India: the Central India Eye and Medical Study. Acta Ophthalmol 2012;90:e649-50.

3. $X u L, H u L, M a K, L i J$, Jonas JB. Prevalence of retinitis pigmentosa in urban and rural adult Chinese: The Beijing Eye Study. European journal of ophthalmology 2006;16:865-6.

4. Dias MF, Joo K, Kemp JA, et al. Molecular genetics and emerging therapies for retinitis pigmentosa: Basic research and clinical perspectives. Prog Retin Eye Res 2018;63:10731.

5. Ameri H. Prospect of retinal gene therapy following commercialization of voretigene neparvovec-rzyl for retinal dystrophy mediated by RPE65 mutation. J Curr Ophthalmol 2018;30:1-2.

6. Anderson D. Standard Perimetry. Ophthalmol Clin N Am 2003: 205-12.

7. J G. Le scotome annulaire dans la degenerescence pigmentaire de la retine. Ann d'Ocul 1901;125:101-30.

8. Grover S, Fishman GA, Anderson RJ, Alexander KR, Derlacki DJ. Rate of visual field loss in retinitis pigmentosa. Ophthalmology 1997;104:460-5.

9. Marmor MF. Visual acuity and field loss in retinitis pigmentosa. Arch Ophthalmol 1991;109:13-4.

10. Berson EL, Sandberg MA, Rosner B, Birch DG, Hanson AH. Natural course of retinitis pigmentosa over a three-year interval. Am J Ophthalmol 1985;99:240-51.

11. Hirakawa H, lijima H, Gohdo T, Imai M, Tsukahara S. Progression of defects in the central 10-degree visual field of patients with retinitis pigmentosa and choroideremia. Am J Ophthalmol 1999;127:436-42.

12. Swanson WH, Felius J, Birch DG. Effect of stimulus size on static visual fields in patients with retinitis pigmentosa. Ophthalmology 2000;107:1950-4. 
13. Granse L, Ponjavic V, Andreasson S. Full-field ERG, multifocal ERG and multifocal $V E P$ in patients with retinitis pigmentosa and residual central visual fields. Acta Ophthalmol Scand 2004;82:701-6.

14. Nagy D, Schonfisch B, Zrenner E, Jagle H. Long-term follow-up of retinitis pigmentosa patients with multifocal electroretinography. Invest Ophthalmol Vis Sci 2008;49:4664-71.

15. Falsini B, Galli-Resta L, Fadda A, et al. Long-Term Decline of Central Cone Function in Retinitis Pigmentosa Evaluated by Focal Electroretinogram. Invest Ophthalmol Vis Sci 2012;53:7701-9.

16. Apushkin MA FG, Alexander KA, and Shahidi M. Retinal thickness and visual thresholds measured in patients with retinitis pigmentosa. Retina 2007;27:349-57.

17. Witkin AJ, Ko TH, Fujimoto JG, et al. Ultra-high resolution optical coherence tomography assessment of photoreceptors in retinitis pigmentosa and related diseases. Am J Ophthalmol 2006;142:945-52.

18. Rangaswamy NV, Patel HM, Locke KG, Hood DC, and Birch DG. A comparison of visual field sensitivity to photoreceptor thickness in retinitis pigmentosa. Invest Ophthalmol Vis Sci 2010;51:4213-9.

19. Hood DC, Lazow MA, Locke KG, Greenstein VC, Birch DG. The transition zone between healthy and diseased retina in patients with retinitis pigmentosa. Invest Ophthalmol Vis Sci 2011;52:101-8.

20. Fischer MD FJ, Gillies MC, Sutter FK, Helbig H, and Barthelmes D. A new method to monitor visual field defects caused by photoreceptor degeneration by quantitative optical coherence tomography. Invest Ophthalmol Vis Sci 2008;49 3617-21

21. Hood DC, Ramachandran R, Holopigian K, Lazow M, Birch DG, Greenstein VC. Method for deriving visual field boundaries from OCT scans of patients with retinitis pigmentosa. Biomed Opt Express 2011;2:1106-14.

22. Birch DG, Locke KG, Wen Y, Locke KI, Hoffman DR, Hood DC. Spectral-domain optical coherence tomography measures of outer segment layer progression in patients with X-linked retinitis pigmentosa. JAMA Ophthalmol 2013;131:1143-50.

23. Mitamura Y, Mitamura-Aizawa S, Katome T, et al. Photoreceptor impairment and restoration on optical coherence tomographic image. J Ophthalmol 2013;2013:518170.

24. Ramachandran R, Zhou L, Locke KG, Birch DG and Hood DC. A Comparison of Methods for Tracking Progression in X-Linked Retinitis Pigmentosa Using Frequency Domain OCT. Trans/ Vis Sci Technol 2013;2:5.

25. Birch DG WY, Locke $K$, Hood DC. Rod sensitivity, cone sensitivity, and photoreceptor layer thickness in retinal degenerative diseases. Invest Ophthalmol Vis Sci 2011;52:7141-7. 
26. Wen Y, Birch DG. Outer Segment Thickness Predicts Visual Field Response to QLT091001 in Patients with RPE65 or LRAT Mutations. TransI Vis Sci Technol 2015;4:8.

27. Robson AG, El-Amir A, Bailey C, et al. Pattern ERG correlates of abnormal fundus autofluorescence in patients with retinitis pigmentosa and normal visual acuity. Invest Ophthalmol Vis Sci 2003;44:3544-50.

28. Robson AG, Saihan Z, Jenkins SA, et al. Functional characterisation and serial imaging of abnormal fundus autofluorescence in patients with retinitis pigmentosa and normal visual acuity. Br J Ophthalmol 2005;90:472-9.

29. Lima LH, Cella W, Greenstein VC, et al. Structural assessment of hyperautofluorescent ring in patients with retinitis pigmentosa. Retina 2009;29:1025-31.

30. Wakabayashi T, Sawa M, Gomi F, Tsujikawa M. Correlation of fundus autofluorescence with photoreceptor morphology and functional changes in eyes with retinitis pigmentosa. Acta Ophthalmol 2010;88:e177-83.

31. Acton JH, Smith RT, Greenberg JP, Greenstein VC. Comparison between MP-1 and Humphrey visual field defects in glaucoma and retinitis pigmentosa. Optom Vis Sci 2012;89:1050-8.

32. Aizawa S, Mitamura Y, Hagiwara A, Sugawara T, Yamamoto S. Changes of fundus autofluorescence, photoreceptor inner and outer segment junction line, and visual function in patients with retinitis pigmentosa. Clin Exp Ophthalmol 2010;38:597-604.

33. Lenassi E, Troeger E, Wilke R, Hawlina M. Correlation between macular morphology and sensitivity in patients with retinitis pigmentosa and hyperautofluorescent ring. Invest Ophthalmol Vis Sci 2012;53:47-52.

34. Battu R, Khanna A, Hegde B, Berendschot TT, Grover S, Schouten JS. Correlation of structure and function of the macula in patients with retinitis pigmentosa. Eye (Lond) 2015;29:895-901.

35. Igarashi N, Matsuura M, Hashimoto $Y$, et al. Assessing Visual Fields in Patients with Retinitis Pigmentosa Using a Novel Microperimeter with Eye Tracking: The MP-3. PloS one 2016;11:e0166666.

36. Battu R, Dabir S, Khanna A, Kumar AK, Roy AS. Adaptive optics imaging of the retina. Indian J Ophthalmol 2014;62:60-5.

37. Lombardo M, Serrao S, Devaney N, Parravano M, Lombardo G. Adaptive optics technology for high-resolution retinal imaging. Sensors 2012;13:334-66.

38. Duncan JL, Zhang Y, Gandhi J, et al. High-resolution imaging with adaptive optics in patients with inherited retinal degeneration. Invest Ophthalmol Vis Sci 2007;48:3283-91.

39. Makiyama $Y$, Ooto $S$, Hangai M, et al. Macular cone abnormalities in retinitis pigmentosa with preserved central vision using adaptive optics scanning laser ophthalmoscopy. PloS one 2013;8:e79447. 
40. Tojo N, Nakamura T, Fuchizawa C, Oiwake T, Hayashi A. Adaptive optics fundus images of cone photoreceptors in the macula of patients with retinitis pigmentosa. Clin Ophthalmol 2013;7:203-10.

41. Menghini M, Lujan BJ, Zayit-SoudryS, et al. Correlation of outer nuclear layer thickness with cone density values in patients with retinitis pigmentosa and healthy subjects. Invest Ophthalmol Vis Sci 2015;56:372-81.

42. Sun LW, Johnson RD, Langlo CS, et al. Assessing Photoreceptor Structure in Retinitis Pigmentosa and Usher Syndrome. Invest Ophthalmol Vis Sci 2016;57:2428-42.

43. Alnawaiseh M, Schubert F, Heiduschka P, Eter N. Optical Coherence Tomography Angiography in Patients with Retinitis Pigmentosa. Retina 2017.

44. Battu R, Mohan A, Khanna A, Kumar A, Shetty R. Retinal oxygen saturation in retinitis pigmentosa and macular dystrophies in asian-Indian eyes. Invest Ophthalmol Vis Sci 2015;56:2798-802.

45. Takagi S, Hirami Y, Takahashi M, et al. Optical coherence tomography angiography in patients with retinitis pigmentosa who have normal visual acuity. Acta Ophthalmol 2018.

46. Chung DC, McCague S, Yu ZF, et al. Novel mobility test to assess functional vision in patients with inherited retinal dystrophies. Clin Exp Ophthalmol 2017.

47. Lorenz B, Wegscheider E, Hamel C, Preising MN, Stieger K. Spatially Resolved Spectral Sensitivities as a Potential Read-out Parameter in Clinical Gene Therapeutic Trials. Ophthalmic Res 2017;58:194-202. 


\section{GENERAL DISCUSSION}





\section{Introduction}

\section{Disease Description}

Retinal cells are interconnected neuronal cells that are responsible for initial processing of visual information. Relentless, slow progressive degeneration of one or more layers of the retina causes a set of blinding disorders- Inherited retinal dystrophies (IRD). Most of these diseases occur due to a single mutation, and form the spectrum of nonsyndromic IRDs. These mutations may be either in the photoreceptors or the retinal pigment epithelium. Based on the mutation, severity, age of clinical presentation, mode of inheritance (autosomal dominant, recessive or x-linked), clinical phenotype, rate of progression, these diseases are classified into various categories. One of the IRDs, Retinitis Pigmentosa (RP) is the most prevalent and a major cause of disability and blindness worldwide. ${ }^{1}$

\section{Genetic causes}

The wide heterogeneity of the disease is evident by the large number of genetic mutations associated with IRDs. The Retinal Information Network (Retnet, https://sph. uth.edu/retnet/) has noted over 300 genes associated with IRDs as on January 2018. ${ }^{2}$ Each of these genes encodes a protein that plays a vital role in the visual processes that occur either in the neuroretina and / or RPE. Mutations in these genes causes abnormalities in amino acids and subsequent protein formation, leading to a cascade of reactions that eventually ends in tissue injury, apoptosis, and tissue remodeling. ${ }^{1}$

Identification of these genes underlying IRDs is of immense interest to clinicians and scientists. Methods to identify the underlying genetic mutations have advanced significantly over the years. ${ }^{3}$ While targeted panel sequencing tests look for specific genes involved, exome sequencing tests all coding regions (i.e., exons) within the human genome. A gene filter containing all the IRD-related genes is further used to limit the risk of incidental findings, not pertinent to the IRD. There is an advantage to exome sequencing: - if the causative gene is not identified in any of the known genes, other potential genes can be explored, thus identifying novel disease-causing genes. Whole genome sequencing is increasingly being utilized, although determining the functional role of many of these newer genes will be a challenge. 


\section{Treatment}

Treatment of IRDs has entered an era from being incurable to having emerging therapeutic options. Many pharmacological agents have been tried in the treatment of IRDs including neurotrophic factors, antioxidants and anti-apoptotic agents, although their efficacy has not yet been proven. ${ }^{4}$ Current therapies for various IRDs include ongoing or completed clinical trials of gene therapy, cell therapy and retinal prostheses.

\section{Gene Therapy}

The US Food and Drug Administration (FDA) approval of voretigene neparvovec-rzyl (Luxturna) for RPE-65 associated retinal degeneration in December 2017 is a milestone in the treatment of IRDs, heralding a new era in the management of these diseases. ${ }^{5}$ This is the first approved ocular gene therapy biologic product. Gene therapy is the strategy to correct genetic defects using a viral or non-viral vector that replaces or silences the causative gene. Although a very promising modality for the treatment of IRDs, gene therapy has limitations. ${ }^{6}$ Gene therapy is gene specific, and a new treatment for each gene has to go through all the steps of drug development including animal studies, clinical trials and regulatory processes, and is therefore inevitably very expensive. Gene therapy requires viable cells and it is unclear at this point whether the therapy can prevent further progression of the disease. Till further developments occur, the method of delivery is invasive and carries the associated complications of the procedure.

\section{Stem Cell Therapy}

Stem cell science is advancing, and although early, offers unprecedented opportunities for cell replacement strategies. ${ }^{7}$ Stem cells are characterized by three general properties: their capacity for unlimited self-renewal, their unspecialized status, and their ability to differentiate into multiple cell types. Although most of the early research was conducted using embryonic stem cells (ESC), induced pluripotent cells (iPSC) is making headway in current research. Obtained from peripheral blood or a skin biopsy, iPSC offers an easy approach compared to ESC. Derivation and differentiation of human iPSC into RPE, photoreceptors and retinal organoids invitro provides exciting opportunities for cell-replacement therapy and screening small molecules for therapeutic potential. To achieve an effective transition from research and early-phase clinical trials to generalized treatment using human iPSCs, there are several processes that need to be optimized. These include optimizing differentiation efficiency and 
reproducibility across cell lines, overcoming immune-mediated difficulties in response to allogenic transplants and challenges associated with generation of current good manufacturing practices (GMPs). ${ }^{8}$

\section{Retinal Prosthesis}

Retinal prosthesis was the first approved therapy for IRDs. The Argus-II was the first US-FDA approved retinal implant while the subretinal implant was approved for use in Europe. Continued improvement in visual acuity has been seen with increasing number and density of electrodes in both the implants. ${ }^{9,10}$

\section{Timing of Interventions}

While research into various treatment options continues, one of the major hurdles facing scientists and clinicians is the appropriate time of intervention in these patients. ${ }^{11}$ An ideal time frame would be once the disease pathogenesis and progression has been established, and before significant vision loss occurs. This raises several questions. Is it possible to quantify photoreceptor/and or RPE loss through various imaging modalities? And to what extent do these modalities overlap or show a different spectrum of the disease stage? For example, pretreatment evaluations of the RPE- 65 gene therapy trials showed that despite the observed retained photoreceptors studied using OCT and darkadapted sensitivity, vision loss was severe. ${ }^{12}$ This shows that there may a discrepancy between structure and function. It is therefore important to identify and treat a disease before visual potential is lost. There are over a 100 clinical trials listed on clinicaltrials. gov addressing treatment of RP and over 20 in Stargardt disease currently. ${ }^{13} \mathrm{All}$ of these have to assess the correct stage to intervene based on current imaging and functional assessment modalities.

\section{Timing Based on Imaging and Functional Assessment}

Currently, photoreceptor measurement on the OCT and estimation of the RPE integrity through the fundus autofluorescence are the commonest tools available in an ophthalmic clinic to assess integrity of the photoreceptor/RPE layer in IRDs. Corresponding functional measurements can be made through microperimetry that can assess function in the same small local area in a more refined way as compared to traditional perimetry.

Although there is a flux of imaging modalities, the current status of these technologies to assess viability of cells for new treatments is at best approximate. We do not know 
how much of the outer nuclear layer/RPE needs to be retained for the results of new therapies to be successful. We do not know how soon and how much therapy needs to be instituted to ensure that noticeable vision loss does not occur at all. Just like therapeutic clinical trials, progress in imaging is evolving.

\section{Assessment of Outcome in New Therapy Trials}

The above-discussed imaging modalities can also be used to assess outcome in new IRDs trials. However, in many phase one clinical trials, selected patients have very poor functional vision, where a noticeable change may not be possible using traditional methods of visual assessment. To this end, to utilize a more patient relevant functional outcome other assessments have been used to obtain FDA approval. The trials that led to the approval of voretigene neparvovec-rzyl (Luxturna) gene therapy for RPE65related retinal dystrophies used standardized multi-luminance mobility testing (MLMT) as the outcome measure, a novel end point that tracks functional vision changes in patients with IRDs over time. ${ }^{14}$ Similarly, the outcome measures used in the Argus II epiretinal prosthesis included two functional visual outcomes- the square localization test, which measured the patient's ability to localize a white square on a black touch-screen monitor and the direction-of-motion test, which measured the patient's ability to detect motion. ${ }^{15}$

\section{Contribution of this Thesis to Knowledge Base of IRDs}

This thesis addresses some of the newer imaging modalities and emerging genetic data in IRDs.

The first part of the thesis- Chapters 3-6 analyses some of the imaging techniques that are currently in use in the management of IRDs. Progress in the management of IRDs has gone hand in hand with technological breakthroughs. From the era of fundus photography and fundus fluorescein angiography, imaging has progressed through optical coherence tomography, fundus autofluorescence, adaptive optics, scanning laser ophthalmoscope, microperimetry, macular pigment optical density, oximetry and OCT-angiography.

The response of human photoreceptors to light is extremely dynamic, and imaging them in an intact retina is a challenge. Adaptive optics is one such developing technology that allows imaging of the cone and possibly, rod photoreceptors. In this thesis we have described the basics of adaptive optics and its advantages over current conventional methods of imaging. SD-OCT provides an excellent lateral resolution 
while Adaptive optics enhances the axial resolution considerably. It therefore allows imaging of the photoreceptors esp cones, blood vessels and the optic nerve head. In IRDs, AO provides an objective and sensitive measure of photoreceptor survival. This allows early identification of their damage, ability to narrow the time frame when treatment can be instituted and provide an objective measurement of treatment response. In very early $A B C A 4$-associated retinopathy, $A O$ is able to identify changes in cone spacing before clinical symptoms develop. ${ }^{16}$ This is encouraging as it allows early application of treatment options as new therapies become readily available to prevent vision loss.

Optical coherence tomography (OCT) has evolved from time domain OCT to spectraldomain OCT and the newer swept source OCT. These are being combined with Adaptive optics and scanning laser ophthalmoscope to attain higher axial and lateral resolution to create platforms that is able to image single photoreceptor cells. ${ }^{17}$

While OCT measures the structural changes, evaluating functional changes is equally essential. Microperimetry (MP) measures point-to-point retinal sensitivity. MP has progressed from the Nidek MP-1 to MAIA. In our studies we observed that the retinal sensitivity in RP showed a significant correlation with the outer retinal thickness. However, the overlap between OCT values and microperimetry values do not correspond perfectly. This implies that both need to be assessed to give a multidimensional picture of IRDs and the outcome of clinical trials. Such an approach will give better opportunities to predict the outcome more accurately and understand mechanisms of obtaining certain outcomes. Moreover, it is of value to quantify the amount of outer retinal thickness that leads to a lower microperimetry value thereby showing the amount of outer retinal thickness that needs to be retained to leave its functional value unaffected.

Measurement of the vasculature has been of immense interest in IRDs, both to understand the pathogenesis and monitor progression. It has become possible to measure the oxygen saturation through retinal oximetry and scanning laser Doppler flowmetry and analyse the superficial and deep retinal vessels through OCT-angiography. In this thesis, we have shown significant narrowing of both arterioles and venules and a higher arteriovenous saturation difference in RP on the oximetry. Arterial vessel density is a hallmark of IRDs like RP. The information obtained from oximetry in various IRDs continues to evolve; it is possible that oxygen saturation and vascular changes may be one of the earliest predictors of cell loss in RP, thus allowing earlier applications of new therapies. 
Noninvasive measurements of ultra microstructures and microvasculature of the retina is a major advance in imaging the human retina. With artificial intelligence and machine learning on the horizon, it is exciting to foresee better understanding of the disease pathogenesis and more definitive monitoring of the disease progression and response to treatment.

The second part of the thesis- chapters 7-9 demonstrates some of the spectrum of phenotypes and their associated genetic mutations in India. Molecular genetic diagnosis and genotyping patients with IRDs help arrive at a specific diagnosis, allows prognostication and helps explore the possibilities of treatment. Given the large burden of IRDs in India, there are relatively fewer mutational reports from across the country. The need to study genotypes in India specifically is emphasized by the high prevalence of consanguinity amongst the population, and the more common prevalence of autosomal recessive diseases as a result of this. It is possible therefore that the mutation spectrum of IRDs in India is different from that reported from the rest of the world. It therefore becomes imperative to conduct molecular genetic screening on a larger number of patients with IRDs, to enable better understanding of the disease in the Indian context.

Following RP, Stargardt disease is the most common IRD seen in India. However there is very little literature on Stargardt disease from India. Chapter seven discusses the mutational spectrum seen in this disease, and identifies two new mutations in this population. Both the new mutations, a homozygous missense mutation (p.Thr971Asn) and a compound heterozygous change (p.Tyr872X) in association with p.Gly1961Glu were noted to be associated with a severe phenotype.

The Prospective Progression of Atrophy Secondary to Stargardt disease (ProgStar) study was a multicentre prospective/retrospective study of patients with mutationproven Stargardt disease across the United States and Europe. ${ }^{18}$ The main aim of the study was to characterize the natural history of disease progression using a variety of structural and functional measures including FAF, SD-OCT and MP. The studies were also designed to assess risk factors for the development and progression of atrophic changes in patients with ABCA4 -related Stargardt disease. The results of several aspects of the study have been published. ProgStar 2 concluded that visual acuity alone is unlikely to be sensitive outcome measure for treatment trials of Stargardt disease, given the slow rate of visual acuity loss; this was further reiterated in ProgStar6. ${ }^{19,20}$ ProgStar 7 studied a newer outcome measure, retinal sensitivity on 
the MP while ProgStar 9 studied FAF as outcome measures. ${ }^{21,}{ }^{22}$ Both concluded that retinal sensitivity testing on MP and FAF may provide important outcome measures in future clinical trials. ProgStar studies have helped significantly in better understanding the natural history, genotypes and outcome measures for clinical trials.

Inspite of characteristic phenotype, molecular heterogeneity can sometimes be surprising and lead to more complex considerations into the pathogenesis of the disease. Neuronal Ceroid Lipofuscinosis ( $\mathrm{NCL}$ ) is one such disease with a wide variety of genetic mutations and fairly homogeneous phenotype. As the cost of genetic testing across the world decreases, whole exome/whole genome sequencing becomes the standard to identify newer mutations, which will be especially appropriate in genetic diseases with a more homogenous phenotype but heterogenous genotype. The role of genetic counselling/genetic counsellors cannot be emphasized more in such a case. Appropriate genetic counselling at an opportune time can prevent multiple members in a family being affected with severe disease.

There is no single imaging modality that can used to assess disease progression or be used in clinical trials for prognostication. In view of the severe visual loss that is already present in most patients included in the various clinical trials currently, functional assessment of vision and its structural correlation is a challenge. Most of the treatment modalities/clinical trials are still in their infancy, and therefore clinicians/researchers have to be cautious in including patients for these trials. Most of these treatments, both approved and in clinical trials, have a fairly significant risk of complications and the risk of further visual loss in a patient with already poor vision due to the primary retinal degenerative condition. Structure-function correlation becomes a major challenge due to this, and therefore a single imaging modality or a single assessment of functional vision is not possible. A combination of multiple imaging modalities and functional visual assessments need to be done to obtain useful information.

India has its own set of challenges in managing these patients. Given the large population of India, and the continued threat of communicable diseases, it is easy to ignore rare diseases. With several programs across India that cater to cataract-related blindness, hither-to untreatable conditions like inherited retinal diseases are beginning to gain importance. There is no universal definition for rare diseases like IRDs. Most countries define 'rare' or 'orphan' diseases as those affecting less than a specific number of persons in a given population. The World Health Organization has suggested that a rare disease be defined as one with a prevalence less than $6.5-10$ per 10,000 persons. ${ }^{23}$ 
The number used in the USA is 7.5, in Europe 5, in Japan 4, in South Korea 4, Australia 1.1 , as the prevalence per 10,000 population. ${ }^{23}$ Going by this definition, RP with a prevalence of 1 in 4000 in the USA is a rare disease. ${ }^{24}$ Like many developing countries, India has no standard definition for a rare disease. However, given the prevalence of more than 1 in 1000, the prevalence is much higher in India and not within the range of rare disease prevalence as reported in other countries or in the high range of rare diseases as reported by the WHO. ${ }^{25,26}$ India will have to define a rare disease in the context of its own population, prevalence patterns, health care system and resources.

There is a need to create a registry of patients with retinal dystrophies detailing their phenotype and genotype in India. A large number of patients need to undergo molecular diagnosis to enable a better understanding of the mutation spectrum in India. This would enable research into the treatment options that would become available in the near future. As far as gene therapies are concerned that addresses one specific mutation, it is of value to use whole genome sequencing and assess the prevalence of the mutations specifically for India. It is possible that the mutations patterns in India are different compared to other countries e.g. due to consanguinity. This will also help identify mutations that need to be addressed/treated to have the largest impact. In addition, patients need to be assessed several times over many years with multi modal imaging techniques to identify those at risk of functional loss and predict the presence of cells that are still viable.

Appropriate genetic counseling holds the key to successful management of patients with IRDs. Currently, there are very few trained genetic counselors. In addition to their paucity, these counselors face several challenges in managing patients with IRDs in India. Although English and Hindi are the official languages, there are 22 languages recognized by the Indian constitution; language barriers for counseling are significant. Family systems are different with arranged marriages and consanguinity is still in vogue. Extended family members sometime demand disclosure of genetic test results; cultural and religious beliefs are significantly different. The key is to train a larger number of personnel in genetic counseling with a specific focus on IRDs. More centers need to start training genetic counselors. This is especially pertinent given the prospective therapies on the horizon. Well-trained genetic counselors can bridge the gap between the physician/geneticist/visual rehabilitation and become the pivotal point for management of these complex diseases.

Some of the major eye institutions across the country have started addressing these 
needs by creating separate departments of eye genetics within the larger umbrella. These include All India Institute of Medical Sciences, New Delhi; Sankara Nethralaya, Chennai; LV Prasad Eye Institute, Hyderabad; Aravind Eye Hospitals, Madurai; Narayana Nethralaya, Bangalore and the Centre for Eye Genetics and Research, Bangalore. All these departments are well equipped with advanced clinical and molecular diagnostics and low-vision rehabilitation departments. The majority of the research/publications in eye/retinal genetics are from these centers. These centers have also started their initial foray into research towards retinal stem cells and gene therapy.

In summary, this thesis reviews the expanding role of newer imaging systems in the management of inherited retinal diseases. It also adds to the existing literature of genetic mutations in the Indian population. The thesis has a special relevance to patients in India, given the exciting treatment options that are on the horizon and the particular genetic characteristics of this population.

\section{Future Perspectives}

The day of 'Personalized medicine' is not far. Cell therapy can be achieved by replacement of autologous cells that has been treated with gene-editing technologies like CRISPR-Cas (clustered regulatory interspaced short palindromic repeats). ${ }^{27}$ This will then overcome immune response, one of the biggest challenge in patients receiving allogenic transplants. However, personalized medicine is bound to be a very expensive approach. Luxturna, the RPE-65 gene therapy product currently costs about $\$ 450,000$ per injection in the US. ${ }^{28}$ Creation of banks of HLA-matched iPSC universal donors, akin to the current blood banks that store iPSC lines homozygous for a range of HLA types is already on the anvil

While the world is on the cusp of major breakthroughs in the treatment of IRDs, it is important to take cognizance of the fact that the retina is an extremely complicated organ, and research into replacing, re-growing or restoring damaged retina is bound to be a very long and tedious process. For eg. the routine use of intraocular lenses today in cataract surgeries belies the many years of painstaking research in both the materials and processes that it took to achieve the surgical excellence that surgeons can boast of today. However given the current extensive research and immense treatment potential that seems possible, the future looks very promising for patients with retinal degenerative diseases. 


\section{References}

1. Verbakel SK, van Huet RAC, Boon CJF, et al. Non-syndromic retinitis pigmentosa. Progress in Retinal and Eye Research 2018; https://doi.org/10.1016/j. preteyeres.2018.03.005

2. Retnet. https://sph.uth.edu/retnet

3. Stone EM, Aldave AJ, Drack AV, et al. Recommendations for genetic testing of inherited eye diseases. Report of the American Academy of Ophthalmology Task Force on Genetic Testing. Ophthalmology 2012: 119:2408-10

4. Hussain RM, Gregori NZ, Ciulla TA, Lam BL. Pharmacotherapy of retinal disease with visual cycle modulators. Expert Opin Pharmacother 2018; 19:471-481

5. Russell S, Bennett J, Wellman JA et al. Efficacy and safety of voretigene neparvovec (AAV2-hRPE65V2) in patients with RPE65-mediated inherited retinal dystrophy: a randomised, controlled, open-label, phase 3 trial. Lancet 2017; 390: 849-60.

6. Ameri H. Prospect of retinal gene therapy following commercialization of voretigene neparvovec-rzyl for retinal dystrophy mediated by RPE65 mutation. J Curr Ophthalmol 2018; 30: 1-2

7. Sten JH, Tian Y, Funderburgh J, et al. Regenerating eye tissues to preserve and restore vision. Cell Stem Cell 2018; 22: 834-849

8. Canto-Soler V, Flores-Bellver M, and Vergara MN. Stem cell sources and their potential for the treatment of retinal degenerations. Invest Ophthalmol Vis Sci 2016; 57: ORSFd1-9

9. Finn AP, Grewal DS, Vajzovic L. Argus II retinal prosthesis system: a review of patient selection criteria, surgical considerations, and post-operative outcomes. Clin Ophthalmol 2018; 12: 2089-1097

10. Gekeler K, Bartz-Schmidt KU, Sachs H, et al. Implantation, removal and replacement of subretinal electronic implants for restoration of vision in patients with retinitis pigmentosa. Curr Opinion Ophthalmol 2018; 29: 239-47

11. Jacobson SG, Matsui R, Sumaroka A and Cideciyan AV. Retinal structure measurements as inclusion criteria for stem cell-based therapies for retinal degenerations. Invest Ophthalmol Vis Sci. 016;57:ORSFn1-ORSFn9. DOI:10.1167/ iovs.15-17654

12. Jacobson SG, Aleman TS, Cideciyan AV, et al. Identifying photoreceptors in blind eyes caused by RPE65 mutations: prerequisite for human gene therapy success. Proc Natl Acad Sci U S A. 2005;102:6177-6182.

13. www.clinicaltrials.gov 
14. Chung DC, McCague S, Yu S, et al. Novel mobility test to assess functional vision in patients with inherited retinal dystrophies. Clinical and Experimental Ophthalmology 2018; 46: 247-259

15. Rizzo S, Belting C, Cinelli L, et al. The Argus II Retinal Prosthesis: 12-Month outcomes from a single-Study Center. Am J Ophthalmol 2014;157:1282-1290.

16. Song $H$, Rossi EA, Latchney $L$, et al. Cone and rod loss in Stargardt disease revealed by adaptive optics scanning light ophthalmoscopy. JAMA Ophthalmol 2015; 133: 1198203

17. Khan KN, Kasilian M, Mahroo OAR, et al. Early patterns of macular degeneration in ABCA4-associated retinopathy. Ophthalmology 2018; 125: 735-746

18. Strauss RW, Ho A, Munoz B, et al. Progression of Stargardt Disease Study Group. The natural history of the progression of atrophy secondary to Stargardt disease (ProgStar) studies: Design and baseline characteristics: ProgStar Report No. 1 Ophthalmology 2016; 123: 817-28

19. Kong $X$, Strauss RW, Michaelides $M$, et al. Visual acuity loss and associated risk factors in the retrospective progression of Stargardt disease study (ProgStar report No.2). Ophthalmology 2016; 123:1887-1897

20. Kong X, Strauss RW, Cideciyan AV, et al. Visual acuity change over 12 months in the prospective progression of atrophy secondary to Stargardt disease (ProgStar report No 6). Ophthalmology 2017; 124:1640-1651

21. Schönbach EM, Wolfson Y, Strauss RW, et al. Macular Sensitivity Measured With Microperimetry in Stargardt Disease in the Progression of Atrophy Secondary to Stargardt Disease (ProgStar) Study Report No. 7. JAMA Ophthalmol. 2017;135:696703.

22. Strauss RW, Muñoz B, Ho A, et al. Progression of Stargardt Disease as Determined by Fundus Autofluorescence in the Retrospective Progression of Stargardt Disease Study (ProgStar Report No. 9). JAMA Ophthalmol. 2017;135:1232-1241.

23. Song, P, Gao, J, Inagaki, Y, Kokudo, N\& Tang W. Rare diseases, orphan drugs, and their regulation in Asia: current status and future perspectives Intractable and Rare Diseases Research 2012;1:3-9.

24. Bunker $C H$, Berson EL, Bromley WC, Hayes RP RT. Prevalence of retinitis pigmentosa in Maine. Am J Ophthalmol 1984;97:357-65.

25. Sen P, Bhargava A, George R, Ve Ramesh S, Hemamalini A, Prema R, Kumaramanickavel G, Lingam V. Prevalence of retinitis pigmentosa in South Indian population aged above 40 years. Ophthalmic Epidemiol 2008;15:279-81.

26. Nangia V, Jonas JB, Khare A, Sinha A Prevalence of RP in India: the central India Eye and Medical Study. Acta Ophthalmol 2012; 1987:1-2 
27. Sanjurjo-Soriano C and Kalatzis V. Guiding lights in genome editing for inherited retinal disorders: Implications for gene and cell therapy. Neural Plasticity 2018 https://doi.org/10.1155/2018/5056279

28. https://www.cnbc.com/2018/01/03/spark-therapeutics-luxturna-gene-therapy-willcost-about-850000.html 
CHAPTER 13

SUMMARY 

There has been a major thrust across the world in research into treatment of retinal degenerative diseases in recent years. With increase in the number of clinical trials to address the unmet need, outcome measures become important, not only to assess the efficacy of these treatment trials, but also to identify earlier time points where treatment can be instituted. The underlying genetic mutations of each disease are equally important before considering therapeutic interventions, especially gene therapy that is specific to each mutation.

This thesis addresses some of the newer imaging modalities that are being used in the management of IRDs. The thesis also looks into some of the genetic data from India, identifying mutations specific to the continent, and adding to the existing literature.

CHAPTER ONE provides an introduction to retinal dystrophies and outlines the aims of the thesis.

CHAPTER TWO is a detailed review of the current literature on inherited retinal diseases. We have discussed the clinical presentation of the common retinal dystrophies that include retinitis pigmentosa (RP), Stargardt disease and Leber congenital amaurosis. We have also discussed the current status of treatment into these conditions.

CHAPTER THREE is an overview of one of the newer imaging modalities, Adaptive optics (AO). In contrast to the axial resolution provided by optical coherence tomography, $\mathrm{AO}$ is able to provide excellent lateral resolution and therefore identify cellular details including cone count and structure. We have described the structural details noted in normal eyes, myopic eyes and those with retinal dystrophies.

CHAPTER FOUR describes the outer retinal tubules on the AO in patients with Bietti's crystalline dystrophy (BCD). First demonstrated in age-related macular degeneration, these tubules appear as hyporeflective ovoid spaces with hyperreflective borders in the outer nuclear layers on spectral domain optical coherence tomography (SD-OCT). These appear as elongated tubules of varying sizes, as a response to photoreceptor injury. This is the first study to demonstrate and describe these tubules in detail using $A O$ in a retinal dystrophy.

CHAPTER FIVE investigated the structure-function correlation in the macula of patients with retinitis pigmentosa using a custom-designed software. The ultrastructure on the SD-OCT was related to function as measured by microperimetry (MAIA, Macular integrity assessment). We studied the retinal sensitivity at the macula using MAIA and 
the corresponding retinal thickness on SD-OCT, identifying changes that cause a reduction in function.

CHAPTER SIX studied the oxygen saturation profiles in patients with RP and macular dystrophy using the Oxymap T1 retinal oximeter. Arteriolar attenuation is a hallmark of RP. In addition to decrease in vascular diameters, we showed alteration in oxygen saturation profiles in all quadrants in the RP group but mainly in the infero-temporal quadrant in the macular group.

CHAPTER SEVEN studied a cohort of Indian patients with Stargardt disease. We presented the clinical information and identified five mutations in the ABCA4 gene, two of which were novel. This is the first study on genetic analysis of patients with Stargardt disease from India.

CHAPTER EIGHT investigated the results of exome sequencing in a family diagnosed with Neuronal ceroid lipofuscinosis or Batten's disease. NCL is a childhood onset neurodegenerative disease that often presents to the ophthalmologist first. We identified two novel MFSD8 mutations in the family. The chapter discusses the importance of exome sequencing in consanguineous families with neuronal ceroid lipofuscinosis.

CHAPTER NINE studied two Indian families identified with Choroideremia. We studied the clinical presentation and analysed their genetic mutations. SD-OCT showed loss of outer retina, preservation of the inner retina and choroidal thinning in the affected males and retinal pigment epithelial changes in the female carriers. Two known mutations were identified in the CHM gene. This is the first study to describe the genetic analysis of patients with Choroideremia from India.

CHAPTER TEN addresses the challenges of managing patients with retinal dystrophies in India. India faces several difficulties including lack of genetic counsellors and inadequate access to molecular genetic testing. This chapter describes the challenges and efforts to address these and the contribution of associations like the 'Organization for Rare Diseases India' towards addressing these problems.

CHAPTER ELEVEN is a review of various methods used to assess progression in RP. With many clinical trials and therapeutic interventions on the anvil, being able to monitor the progression and identify critical end points for these trials becomes crucial. Several 
relevant outcome measures including visual field assessment, electroretinography, $\mathrm{OCT}$, fundus autofluorescence, microperimetry and $\mathrm{AO}$ were measured. We concluded that multiple modalities are required to arrive at meaningful outcome measures.

In summary, this thesis expostulates the expanding role of newer imaging systems in the management of inherited retinal diseases. It also adds to the existing literature of genetic mutations in the Indian population. The thesis has a special relevance to patients in India, given the exciting treatment options that are on the horizon. 



\section{VALORISATION}

"Who would believe that so small a space could contain the images of all the universe?"

LEONARDO DA VINCI 

Of all the fields of Medicine, advances in technology have impacted Ophthalmology most. The world of retinal diseases is no exception. Vitreoretinal diseases that would have been deemed'inoperable' a few years ago are routinely being done; retinal diseases that never would have been diagnosed, let alone treatable- are being diagnosed with Spectral domain optical coherence tomography (SD-OCT), OCT-Angiography, adaptive optics, microperimetry and treated- with the advent of micro incision vitrectomy surgery for example advanced diabetic tractional retinal detachments, stage 5 retinopathy of prematurity and complex traumas

Genetic diseases of the retina continue to frustrate clinicians (and patients) alike, as they are not amenable to conventional medical or surgical treatment. There is a ray of hope however tenuous at the present time, with the recent approval of gene therapy for RPE-65 related retinal dystrophies, and despite the multiple clinical trials ongoing around the world, the world is still a dark place for patients with these diseases.

As I sat in the outpatient of the retina clinic many years ago, I saw this consanguineous couple with a young child, who had progressive visual failure and seizures. Over the next few weeks, as we informed the mother of the diagnosis of Batten's disease (Neuronal ceroid Lipofuscinosis) in her child, the mother had a question: This was her fourth child; she now had three of her four children affected with this blinding and fatal condition. Why hadn't any doctor told her about this before? Could we have prevented this condition in her children? In chapter 8 the basis for this opportunity to diagnose Neuronal ceroid Lipofuscinosis based on exome sequencing is described.

This opened up the world of 'retinal dystrophies' and 'ophthalmic genetics' to me. As I got more interested, I began to see more and more patients with degenerative diseases of the retina.

My journey into the fascinating world of retinal dystrophies had begun. I knew this was an area of research that I wanted to pursue.

Retinitis pigmentosa is the commonest retinal dystrophy. The prevalence of RP in India is more than 1 in 1000, compared to that of 1 in 4000 in the USA. That puts the number at close to 1.5 million affected with RP alone in India. This is not even considering other retinal dystrophies like Stargardt disease or LCA. Based on the prevalence alone this was then a socially relevant problem to study. Moreover, it supports the need to study this genetic disease from an Indian perspective and also find specific genetic mutations/ modifier genes fueled also by a high prevalence of consanguinity. 
Was my study economically relevant? Undoubtedly. While there are several programs like the National Program for Control of Blindness, "orphan" diseases like retinal dystrophies receive very little attention. For every person who is blind, there are three people who are visually impaired. The economic burden of blindness in India for the year 1997 was Rs. 159 billion (US\$ 4.4 billion). While $23.5 \%$ of the blind in the world live in India, there are no definite statistics about blindness/visual impairment due to retinal degenerative diseases. ${ }^{1,2}$

As I grappled to understand these diseases better, recent technological advances helped me get a better understanding of them. Looking at cone photoreceptors through Adaptive optics or getting better understanding of the blood flow in RP through Oximetry opened up newer insights into the pathogenesis of these diseases as described in Chapters 3 to 6 . It now is possible to see the photoreceptors in vivo and study its density in RP. OCT also gave the opportunity to study the retina in much more detail in vivo in a detail almost similar to that achieved by histological examinations. Moreover, we are now better informed about the relation between the structural changes in Adaptive optics and OCT the function. The latter improved by micro-perimetry as compared to traditional perimetry. Microperimetry shows the sensitivity at a local point and at more points in the center and the opportunity to assess the sensitivity value in exactly the same place after some follow-up time. The sensitivity can therefore be closely related to the local changes in OA and OCT in the same spot.

As disease progression occurs in retinal dystrophies, the retinal tissue reacts to the injury in several ways; one of them is the formation of outer retinal tubules (ORT) described on the SD-OCT. I was curious to see how these tubules appeared on the adaptive optics; whether we could image them at all. Doing simple mathematical calculations based on the location of the ORTs on the SD-OCT, we identified possible locations of the tubules on the Adaptive optics; it was an exciting moment to see long tubules on stitched images of Adaptive Optics as is described in Chapter 4. It had not yet been described in Bietti's Crystalline Dystrophy.

As we started conducting molecular genetic tests on the families presenting at our hospital, it became increasingly clear that there was limited information in the Indian literature regarding retinal dystrophies. While ophthalmic research is at the forefront in India, insights into the molecular genetics of retinal dystrophies was lacking. Towards this end, we published a series of mutations, both already noted and some new to add to the world literature (Chapters 7-9). Managing the staggering number of patients in 
the genetic clinic brought fore the difficulties associated. (Chapter 10) Although we now had clinicians trained in managing patients with IRDs, there were no trained genetic counselors. Managing these patients and their families amidst a busy retina clinic was a challenge in itself. There were very few laboratories that were doing genetic mutational work in IRDs. Talking to multiple labs, discussing with them the importance of starting to look at eye/retina related genes, correlating them to the clinical phenotype- all taught me invaluable lessons.

At Narayana Nethralaya, we hosted a two-day international symposium titled 'SIGNSymposium on Inherited Retinal Disease, Genetics and Newer treatments in 2013. This was a combination of presentations in basic genetics and clinical discussions. We were fortunate to have world leaders in the field of Ophthalmic Genetics come and address the sessions. And I continued to learn!

There is a surge in research into retinal dystrophies in the last decade. This thesis adds significantly to the existing literature about retinal dystrophies in India. With the many elements of research - be it gene therapy, stem cell research, retinal prostheses, small molecules, pharmaceutical therapy- we need more data about the spectrum of retinal dystrophies in India and its impact on the affected people. Once more data is gathered on the clinical phenotype, the number of affected people, the specific genetic mutations in the Indian population- it is then possible to start focusing on the therapies possible. Clinical trials in IRDs can then be started in India to look at multiple treatment options.

Retinal prostheses- both epiretinal and subretinal have been approved for use today. ${ }^{3}$ The USFDA approval of Luxturna- voretigene neparvovec-rzyl for RPE-65 disease developed by Spark therapeutics, USA is a very exciting landmark in the treatment of retinal dystrophies. However, gene therapy poses a very expensive prospect for affected patients. Each injection of Luxturna costs over $\$ 450,000$ per injection currently in the United States. Although no patient in India has received either of these treatments yet, the development of both retinal prosthesis and gene therapy product is a remarkable achievement for science. In addition, both these therapies can behave like platform technologies based on which further products can be developed.

There are several ophthalmic institutions in India doing research in this area - RP Centre for Ophthalmic Sciences, New Delhi, LV Prasad Eye Institute, Hyderabad, Sankara Nethralaya, Chennai, Aravind Eye Hospitals, Madurai, Narayana Nethralaya, Bangalore. 
My own research has grown beyond the scope of this thesis. The Centre for Eye Genetics and Research in Bangalore (www.cegr.org) has been established with the sole idea of managing patients with retinal dystrophies, and provide the time and professional care these patients deserve. Services provided at CEGR include comprehensive clinical examination to arrive at a correct phenotype, genetic counseling after molecular genetic testing, clinical genetic services, personalized low visual rehabilitation services and collaboration with other organizations like ORDI (Organization for Rare Diseases India). Along with ORDI, CEGR has been organizing 'Racefor7', an annual marathon to raise awareness about rare diseases of the eye.

What began as a small idea about exclusive management of these patients turned out to be the beginning of 'Eyestem' (www.eyestem.com). Eyestem is a retinal stem cell company that focuses on research into retinal degenerative diseases. It is incubated at CCamp (Centre for Cellular and molecular platforms), within the NCBS (National Centre for Biological Sciences) campus, Bangalore, India. Eyestem boasts of a dedicated set of scientists and an exceptional advisory team.

The goal of the company is to be able to replace defective photoreceptors and retinal pigment epithelium in patients with retinal degenerative diseases. Towards this, we have used induced pluripotent stem cells (iPSC) from peripheral blood and differentiated them into Retinal Pigment Epithelium (RPE) and photoreceptors (PR) invitro. ${ }^{4}$ Our first set of animal experiments in dedicated knock out models has begun and we have shown successful integration of RPE cells transplanted subretinally.

Autologous stem cell treatment is possible; however the cell lines naturally carry the same genetic mutation that is in the eye. The combination of iPSC and remarkable genome editing tools like CRSIPR/Cas-9 (Clustered Regularly Interspaced Short Palindromic Repeats) make personalized therapy a reality, where a patient's own cells are corrected and used to replace their degenerating retina. ${ }^{5}$ However this is bound to be a challenging and expensive proposition.

Allogeneic stem cell treatment seems an attractive option to autologous treatment. Although the eye is considered immune-privileged, immune responses is one of the major hurdles for allogenic therapy. Systemic and local immunosuppression is administered in current trials; this research is still in its early stages. HLA matched allogeneic stem cell banks that are able to produce clinical grade cells seems a definite possibility. 
At Eyestem, we are aware of challenges ahead. Some of these include overcoming immune responses, difficulties associated with integration of our cells within the host retina, clinical challenges of identifying critical end points in the disease and subretinal administration of the cells and associated regulatory hurdles. However, as science stands at the cusp of major breakthroughs in all these areas across the world, we are quite hopeful that we should be able to bring our current research to the bedside and be able to treat the many affected patients.

To quote the famous French mathematician François Arago

"To get to know, to discover, to publish- this is the destiny of a scientist"

\section{Suggested Reading}

1. Estimation of blindness in India from 2000 through 2020: implications for the blindness control policy. Dandona L, Dandona R, John RK. Natl Med J India. 2001 NovDec;14(6):327-34.

2. Shamanna BR1, Dandona L, Rao GN. Economic burden of blindness in India. IJO, 1998

3. Os/wald J, Baranov P. Regenerative medicine in the retina: from stem cells to cell replacement therapy. Ther Adv Ophthalmol 2018 Apr 26;10:2515841418774433.

4. Rathod R, Surendran H, Battu R, Desai J, Pal R. Induced pluripotent stem cells (iPSC)-derived retinal cells in disease modeling and regenerative medicine. $J$ Chem Neuroanat. 2018 Feb 12. pii: S0891-0618(17)30120-5.

5. Singh R, Cuzzani O, Binette $F$, et al. Pluripotent stem cells for retinal tissue engineering: current status and future prospects. Stem Cell Rev 2018 Aug;14(4):463-483. 

CHAPTER 15

ACKNOWLEDGEMENTS 



\section{Acknowledgements}

As in any research that goes from an idea to completion, there is a long list of people who have helped me along the path to complete my doctorate. I remember the day Prof Muralidhar Kanchi, Chief of Anesthesia at Narayana Hrudayalaya, Narayana Health City asked me if I would register for the Mastricht PhD, I am grateful that he thought I was capable of this.

My first inspiration has always been my parents, who taught not to take excellence for granted. They truly believed in the adage that 'success is $99 \%$ perspiration', thank you Amma and Anna. My sister Savitha, who always stayed in the background, and continued to support me. My brother, Dr Ravi Keshavamurthy, a retina surgeon like me, who always believed his sister could achieve anything (!) and continues to inspire me with his 'never-die-attitude'- Thank you both.

My interest in retinal genetics has predated my PhD registration. Dr Mohammed Naveed, Chief Geneticist at Dubai inculcated this deep interest in genetics and opened my eyes to the world of unknown, thank you Dr Naveed. Prof Kumaramanickavel furthered this interest by encouraging me to do a fellowship in Ocular genetics- Thank you sir.

The three-month ICO fellowship at Moorfields Eye Hospital, London, was truly an eye opener. The exciting world of eye genetics and electrophysiology paved way for a lifelong obsession. Thank you to the entire team at Moorfields Eye Hospital, especially, Prof Anthony Moore and Dr Anthony Robson, both of them have continued to support me much beyond my time there. I am also thankful to Dr Elise Heon, who has been a friend and guiding force, for expanding my horizons in ocular genetics beyond my wildest dreams!

I have to especially thank Dr Rohit Shetty, Vice-chairman, Narayana Nethralaya, who encouraged my interest in this niche area. He and Dr Bhujang Shetty, Chairman, Narayana Nethralaya allowed me the three-month of absence from routine clinical work to go and complete my ICO fellowship. Most of the work for this thesis was done at Narayana Nethralaya; my sincere gratitude to both of them; this work would not have been possible but for their support.

I am grateful to Prof C.A.B Webers for his constant guidance, and his patience with me! Dr Jan Schouten- who has been my mentor and guide ever since this journey began- he 
has been a constant source of encouragement - thank you Jan. Dr Tos Berendschotwho alongside Jan made sure I stay on the path to completion- thank you Tos.

My many colleagues and friends at Narayana Nethralaya -Dr Debashish Das, Dr Priya Srinivasan, Dr Padmamalini, Dr Vasudha, Dr Bhanumathi, Dr Sathidevi, Dr Anjani Khanna, Dr Ashwin Mohan- all of who thought I was capable of doing this- thank you to all.

My many friends- Chetana Bhat- who was relentless in making sure I completed my thesis, Dr Rajesh Babu- who was willing to help me any time, Dr Ashwin Mallipatna-for constantly listening to my rants- thank you all, Dr Sherine Braganza- who has always had a ready shoulder for me to lean on, Savitha- who continues to believe that I can do anything (!), Dipti- for constantly teaching me to look at things practically- heart felt thank you to each one of you!

Dr Shyam Vasudeva Rao has played a big role in this thesis- his constant positive attitude that I can take this thesis to completion helped me along throughout-thank you Shyam.

My daughters Aditi and Ashwini have constantly stood by me, encouraging me in times of despair, showering me with affection, proof reading my thesis, withstanding my absences the times I was away- be it a fellowship or conference, who each gave me a memorabilia- every time I went out of Bangalore- worried that I was going to forget them (!) - thank you darlings. You will always remain uppermost in my mindirrespective of where I am.

My biggest thank you goes out to my husband- Dr Ravindra Battu- who taught me not only Ophthalmology, but also great attitude in life! He has stood by me when I nearly gave up the thesis, and has encouraged me to think out of the box constantly. He has shown by example how 'perfect' is the only way to do things. I do not think this research would have been possible without him by my side- Thank you Ravi.

I am grateful to the support staff at Narayana Nethralaya, Bharat, Leela, Deekshit, Krishna, Sivakumar, Puttaswamy and the entire imaging team- they have truly been the effort behind the scenes. Finally, my heart felt thanks goes out to all my patients and their families- I have come to know many of them very closely, I share their joys and tribulations- thank you to each one of you. 



\section{Curriculum Vitae}

Rajani Battu currently is the Medical Director of Centre for Eye Genetics and Research, Bangalore and a senior Consultant in the department of Ophthalmology at Aster CMI Hospital, Bangalore, a multi-specialty tertiary care hospital.

Rajani graduated out of high school and college with top academic honors throughout. She was awarded the National Talent Search Scholarship by the National Council of Educational Research and Training (NCERT), New Delhi for the entire period of her education. The NCERT awards these scholarships based on a national talent search examination to the most talented students in the country.

Rajani did her basic medical training from Bangalore Medical College, Bangalore University and graduated with three gold medals in Ophthalmology. She subsequently did her Master's degree at St. John's Medical College, Bangalore. She passed the International Basic Science Assessment Examination conducted by the International Council of Ophthalmology, the Diplomate of National Board Exams and obtained her fellowship from the Royal College of Surgeons, Edinburgh. She underwent fellowship training in vitreo-retinal surgery at Medical Research Foundation, Sankara Nethralaya, Chennai, India

After her training, Rajani set up the Vitreoretinal department at Jain Nethralaya, Bangalore. She subsequently joined Narayana Nethralaya as a senior consultant in the department of Vitreoretina. During this period, she did a three-month ICO fellowship in "Inherited Retinal Diseases" with Prof Anthony Moore, Moorfields Eye Hospital, London, UK.

On her return to her parent institute, she started to focus on examining patients with retinal degenerative diseases. In 2013, she organized "SIGN- Symposium on Inherited Retinal Disease, Genetics and Newer Treatments', a two-day international symposium with a specific focus on inherited retinal diseases.

Rajani is the Founder-Director of Centre for Eye Genetics and Research (www.cegr.org) in Bangalore. This is a stand-alone organization that is dedicated to managing patients with inherited retinal diseases. Along with a comprehensive ophthalmic examination, the centre offers genetic counseling and advanced low vision rehabilitation services for these patients and their families. 
She is also the co-founder and chief medical officer of Eyestem (www.eyestem. com), a biomedical company dedicated to research into retinal stem cells. Eyestem is incubated at the Centre for Cellular and Molecular Platforms (CCAMP), NCBS-TIFR, Bangalore and has been awarded the prestigious biotechnology ignition grant from BIRAC, India.

Rajani has several national and international paper presentations/invited talks to her credit. She was awarded the Basheer Mekhri Award for the 2nd best paper at the Annual Karnataka Ophthalmic Society meet in Mysore in 2014.

Rajani is very passionate about bringing the best clinical care to patients with inherited retinal disorders. She is working towards bringing the research at Eyestem to the bedside eventually and CEGR becoming a center of excellence for patients with inherited retinal diseases in India and the world.

\section{Publications}

1. Nguyen TT, Poornachandra B, Verma A, Mehta RA, Phalke S, Battu R, Ramprasad VL, Peterson AS, Ghosh A, Seshagiri S.

Next generation sequencing identifies novel disease-associated BEST1 mutations in Bestrophinopathy patients.

Sci Rep. 2018 Jul 5;8(1):10176. doi: 10.1038/s41598-018-27951-8.

2. Rathod R, Surendran H, Battu R, Desai J, Pal Induced pluripotent stem cells (iPSC)- derived retinal cells in disease modeling and regenerative medicine Journal of Chemical Neuroanatomy 2018; 17: 30120-5.

3. Sathi AV, Murthy G, Battu R, Mahendradas P, Dabir S, Chitralekha De. Role of perimetry in the diagnosis and management of retinal/macular disorders. Jan 2016. Chapter 11. In book Practical Perimetry 129-156

4. Battu R, Prasad A, Kanchi M

Perioperative optic neuropathy in patients undergoing off-pump coronary artery bypass graft surgery. Annals of Cardiac Anaesthesia 2014;17(2):92-7. doi: 10.4103/09719784.129823.

5. Rajasimha HK, Shirol PB, Ramamoorthy P, Hegde M, Barde S, Chandru 
V, Ravinandan ME, Ramchandran R, Haldar K, Lin JC, Babar IA, Girisha KM, Srinivasan S, Navaneetham D, Battu R, Devarakonda R, KiniU, Vijayachandra K and Verma IC.

Organization for Rare Disease India (ORDI)- Addressing the Challenges and Opportunities for Indian Rare Diseases' Community.

Genetics Research (Cambridge) 2014; 96: e009-19

6. Yadav NK, Jayadev C, Mohan A, Vijayan P, Battu R, Dabir S, Shetty B and Shetty R. Subthreshold Micropulse Yellow Laser (577 nm) in Chronic Central Serous Chorioretinopathy - safety profile and treatment outcome.

Eye (Lond) 2015; 29(2):258-65. doi: 10.1038/eye.2014.315. Epub 2015 Jan 23

7. Melanoma associated retinopathy: A new dimension using adaptive optics.

Dabir S, Mangalesh S, Govindraj I, Mallipatna A, Battu R, Shetty R

Oman Journal of Ophthalmology 2015; 8(2):125-7

8. Mailankody P, Battu R, Khanna A, Lenka A, Yadav R, Pal PK.

Optical coherence tomography as a tool to evaluate retinal changes in

Parkinson's disease.

Parkinsonism \& Related Disorders 2015; 21(10):1164-9

9. Mathew S, Seetharam R, Kamble R, Battu R

Ethambutol Induced Optic Chaismopathy

Annals of Neurology 12/2012; 72(Supplement S16):S1-S120.

10. Joy SP, Panda S, Kulkarni GB, Pal PK, Chickabasaviah YT, Battu RR Incontinentia pigmenti with sensorimotor polyneuropathy: a novel association. Neurology India 2009; 57(6):813-5.

11. Silicone Oil Study Group of Sankara Nethralaya.

Use of silicone oil in the management of complex retinal detachment--an Indian experience.

International Ophthalmology 2004; 25(3):129-42.

12. Gopal L, D'Souza CM, Bhende M, Fogla S, Ratra D, Shetty NS, Sharma T, Shanmugam MP, Bhende P, Battu RR, Badrinath SS.

Scleral buckling: implant versus explant.

Retina 2003; 23 (5): 636-40

13. Gopal L, Ramaswamy AA, Madhavan HN, Battu RR, Sharma T, Shanmugam MP, Bhende PS, Bhende M, Ratra D, Shetty NS, Rao MK. Endophthalmitis caused by Acinetobacter calcoaceticus. A profile. Indian Journal of Ophthalmology 2003; 51(4):335-40

14. Gopal L, Shanmugam MP, Battu RR, Shetty NS. 
Congenital Retinoschisis: Successful collapse with photocoagulation. Indian Journal of Ophthalmology 2001; 49; 265-66

15. Gopal L, Ramaswamy A, Madhavan HN, Saswade M, Battu RR.

Postoperative endophthalmitis caused by sequestered Acinetobacter Calcoaceticus.

American Journal of Ophthalmology 2000; 129(3); 388-90

16. Battu RR, Biswas J, Jayakumar N, Madhavan HN, Kumarasamy N.

Papilloedema with peripapillary retinal hemorrhages in an AIDS patient with Cryptococcal meningitis.

Indian Journal of Ophthalmology 2000; 48(1): 47-9 



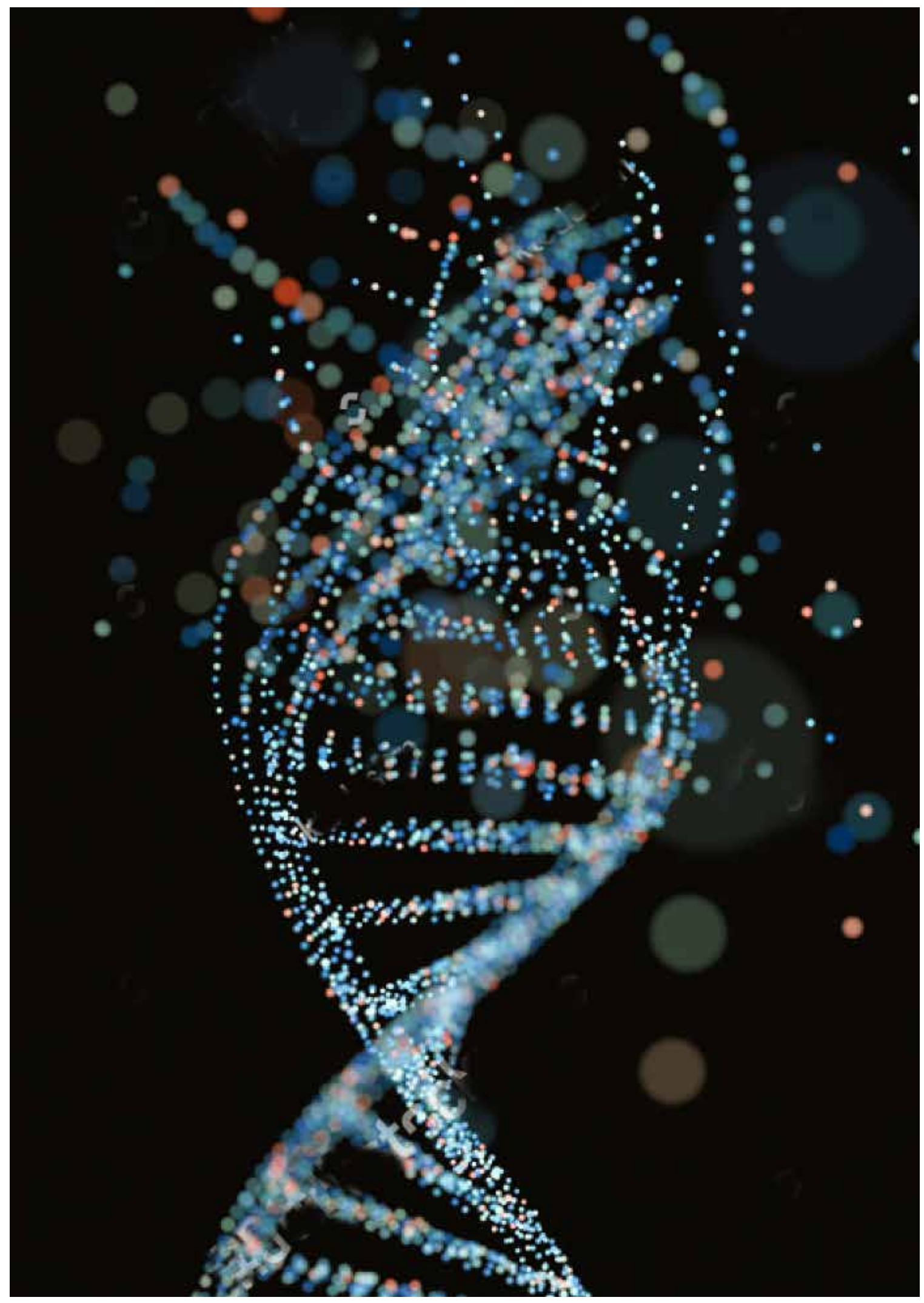

